\title{
The STRIPAK complex and its role in fruiting-body development of the filamentous fungus Sordaria macrospora
}

\author{
Dissertation \\ for the award of the degree \\ “Doctor rerum naturalium” \\ of the Georg-August-Universität Göttingen \\ within the doctoral program Molecular Biology of Cells \\ of the Georg-August University School of Science (GAUSS)
}

\author{
submitted by \\ Stefan Frey \\ from Leer (Ostfriesland)
}

Göttingen, 2015 


\section{Thesis Committee}

Prof. Stefanie Pöggeler

Department of Genetics of Eukaryotic Microorganisms

Institute of Microbiology and Genetics

Prof. Gerhard Braus

Department of Microbiology and Genetics

Institute of Microbiology and Genetics

Prof. Blanche Schwappach

Department of Molecular Biology

University Medical Center Göttingen

Members of the Examination Board

Referee:

Prof. Stefanie Pöggeler

Department of Genetics of Eukaryotic Microorganisms

Institute of Microbiology and Genetics

$2^{\text {nd }}$ Referee:

Prof. Gerhard Braus

Department of Microbiology and Genetics

Institute of Microbiology and Genetics

Further members of the Examination Board

Prof. Blanche Schwappach

Department of Molecular Biology

University Medical Center Göttingen

Prof. Rolf Daniel

Department of Genomic and Applied Microbiology

Institute of Microbiology and Genetics

\section{Prof. Kai Heimel}

Department of Microbial Cell Biology

Institute of Microbiology and Genetics

\section{PD Dr. Michael Hoppert}

Department of General Microbiology

Institute of Microbiology and Genetics

Date of the oral exam: 05.03.2015 


\section{Affirmation}

I hereby declare that this thesis was written independently and with no other sources and aids than quoted.

Göttingen, 23.01.2015

Stefan Frey 
This doctoral study was performed in the group of Prof. Stefanie Pöggeler in the Department of Genetics of Eukaryotic Microorganisms at Institute of Microbiology and Genetics, Georg-AugustUniversity Göttingen.

Some parts of the results section of this doctoral study were peer-reviewed by the journal "Molecular Microbiology” (and are currently under revision) and some results are submitted for publication in the journal "Eukaryotic Cell”.

Stefan Frey, Yasmine Lahmann, Stefanie Pöggeler. Deletion of Smgpi1 encoding a GPI-anchored protein suppresses sterility of the STRIPAK mutant $\triangle$ Smmob3 in the filamentous ascomycete Sordaria macrospora. Molecular Microbiology (in revision)

Author contributions to this publication:

Planned experiments: SP, SF, YL

Performed experiments: SF, YL

Analyzed data: SF, SP

Contributed reagents or other essential material: SP

Wrote the paper: SF, SP

Stefan Frey, Stefanie Pöggeler. Germinal Center Kinases SmKIN3 and SmKIN24 are associated with the Sordaria macrospora Striatin-interacting phosphatase and kinase (STRIPAK) complex. Eukaryotic Cell (submitted)

Author contributions to this publication:

Planned experiments: SP, SF

Performed experiments: SF

Analyzed data: SF, SP

Contributed reagents or other essential material: SP

Wrote the paper: SF, SP 
List of Tables ..................................................................................................................................................... viii

List of Figures................................................................................................................................................ ix

List of Abbreviations ................................................................................................................

Summary ............................................................................................................................... 1

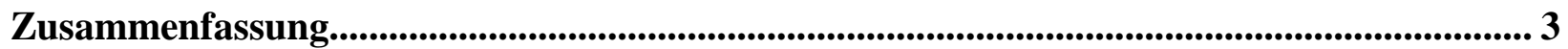

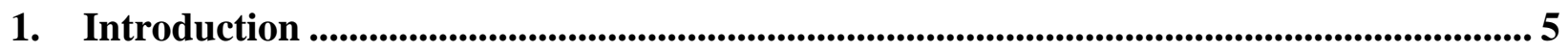

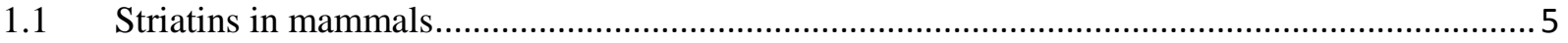

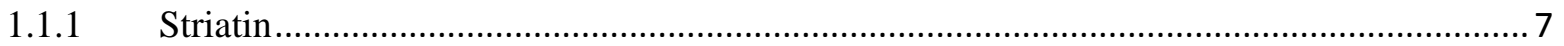

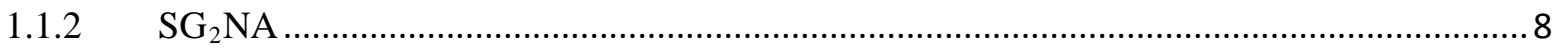

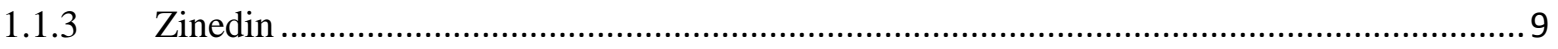

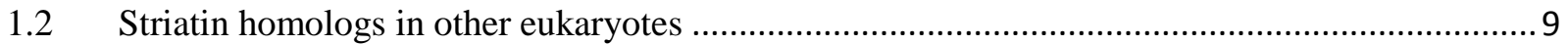

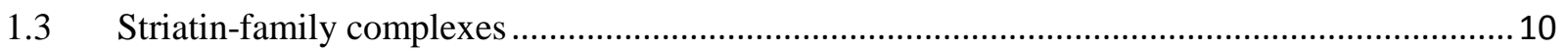

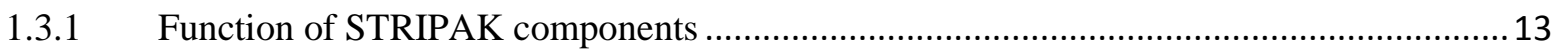

1.3.1.1 Protein phosphatase 2A.......................................................................................... 13

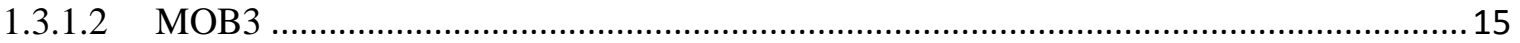

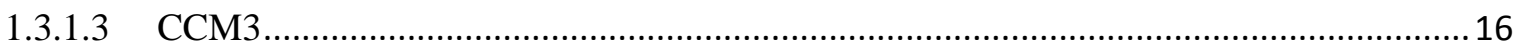

1.3.1.4 The GC III kinases MST4, STK24 and STK25 ….................................................. 19

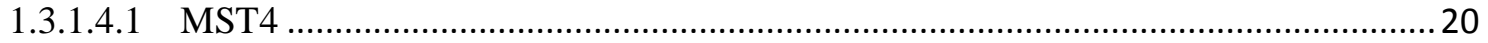

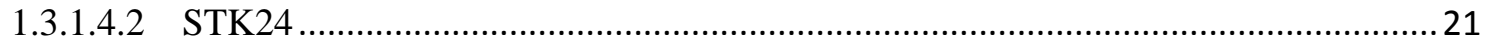

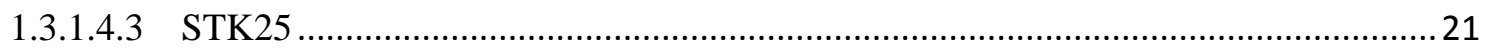

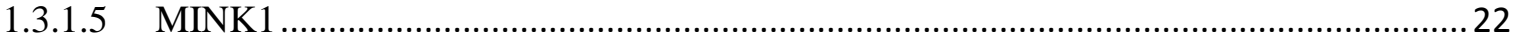

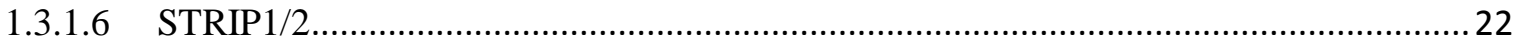

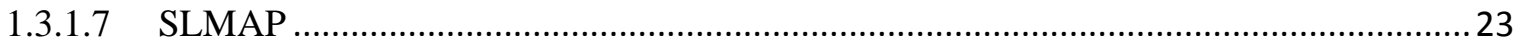

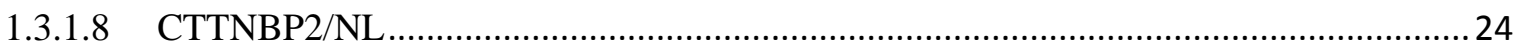

1.3.1.9 SIKE

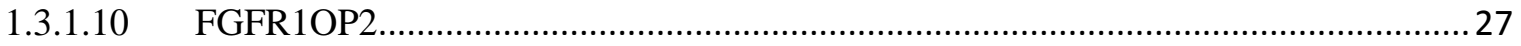

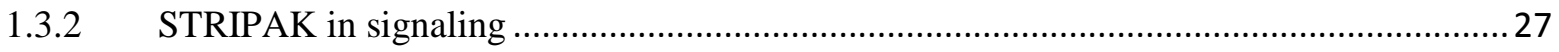

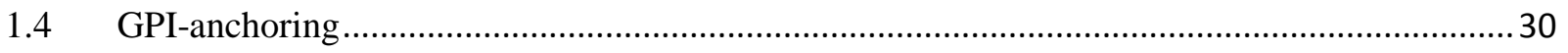

1.5 Sordaria macrospora: A model for fruiting-body development................................................ 33

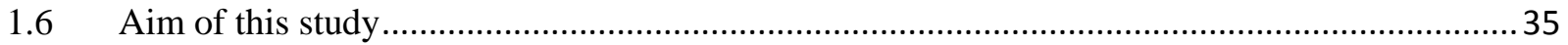


2. Material and Methods ............................................................................................................... 36

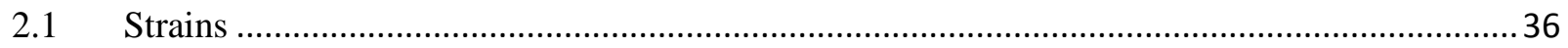

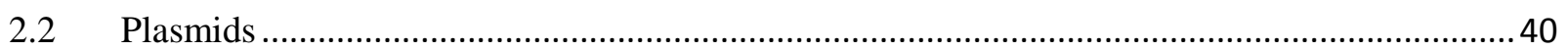

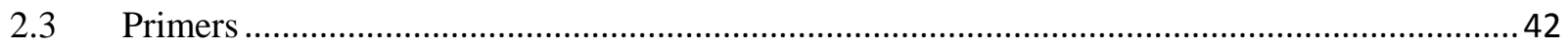

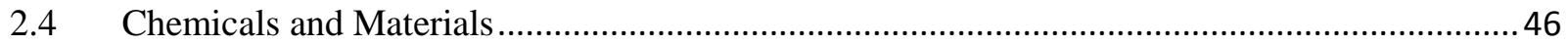

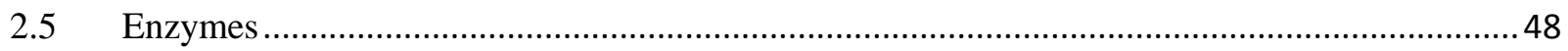

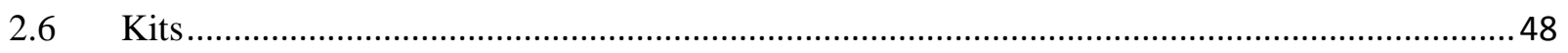

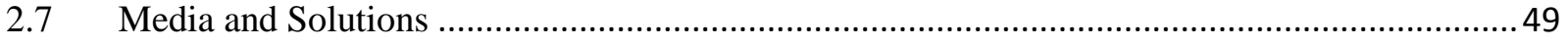

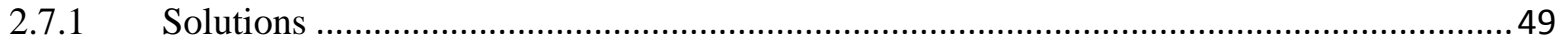

2.7.1.1 Amino-acid stock solutions................................................................................. 49

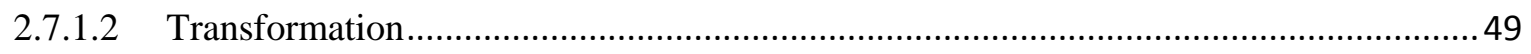

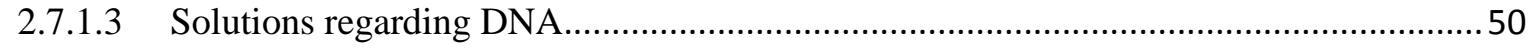

2.7.1.4 Protein regarding solutions ..................................................................................50

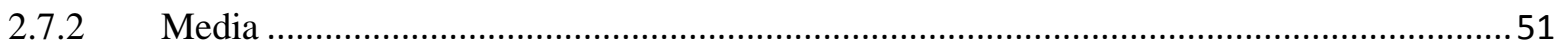

$2.8 \quad$ Strains and culture conditions ......................................................................................... 53

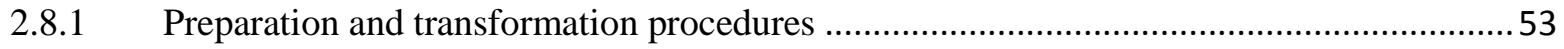

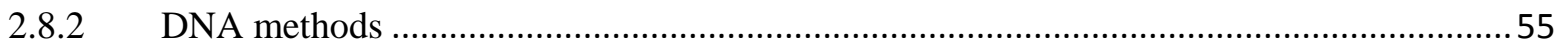

2.8.2.1 Plasmid isolation from E. coli and S. cerevisiae .....................................................55

2.8.2.2 Isolation of RNA and genomic DNA from S. macrospora ..............................................56

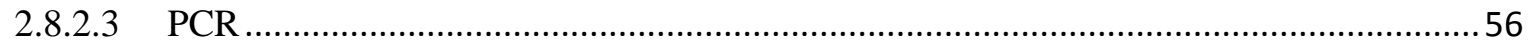

2.8.2.4 Purification of amplified DNA...............................................................................57

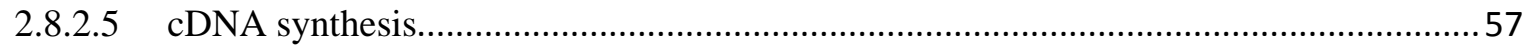

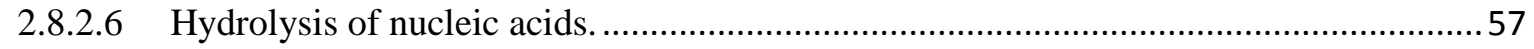

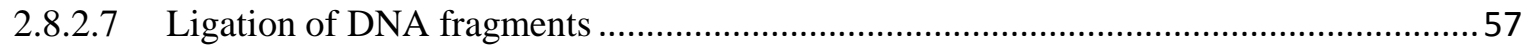

2.8.2.8 Separation of nucleic acids by gel electrophoresis.......................................................58

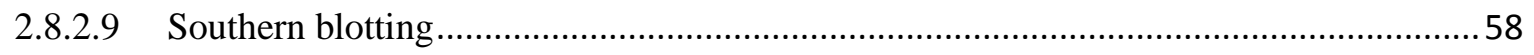

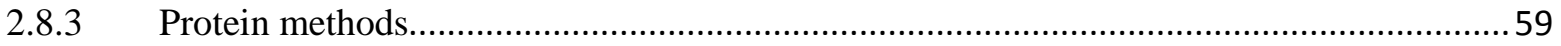

2.8.3.1 S. macrospora protein extraction .........................................................................59

2.8.3.2 Protein concentration measurement .........................................................................59

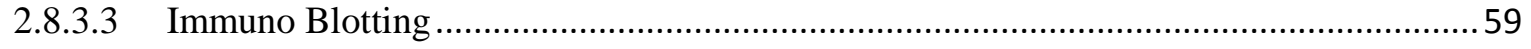

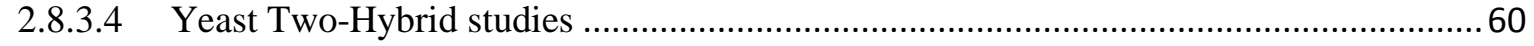

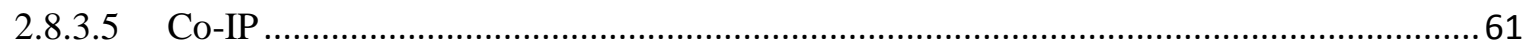




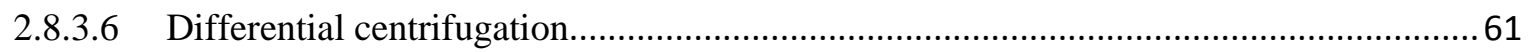

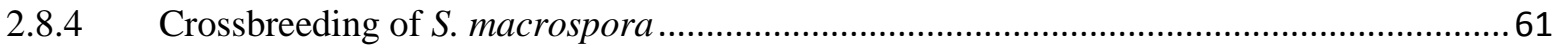

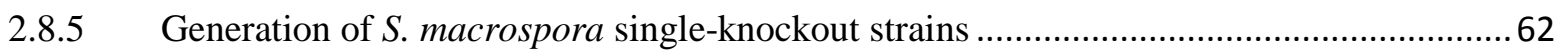

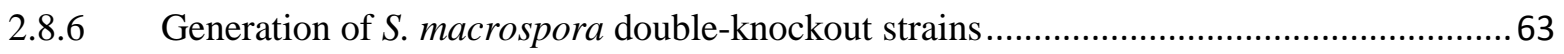

2.8.7 Generation of S. macrospora complementation strains ...................................................63

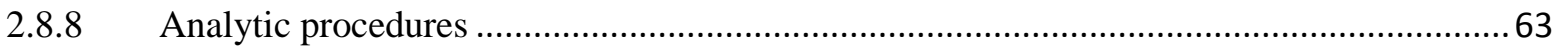

2.8.8.1 Light and fluorescence microscopy investigations .................................................... 63

2.8.8.2 Sequence analysis and oligonucleotide synthesis ...................................................65

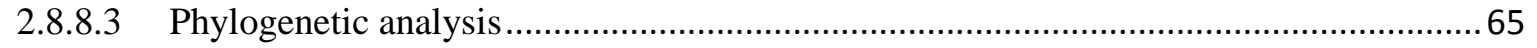

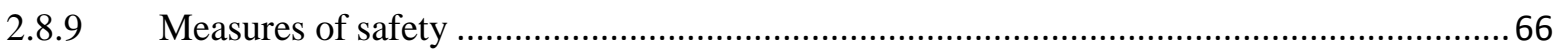

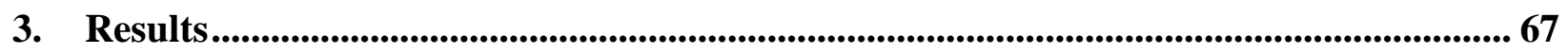

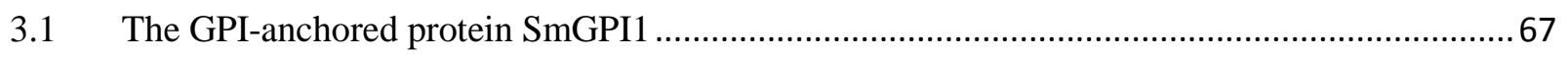

3.1.1 A two-hybrid screen identified a GPI-anchored protein as an interaction partner of

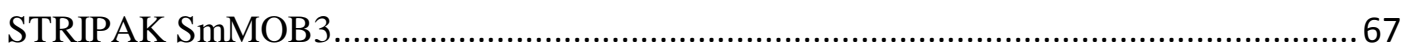

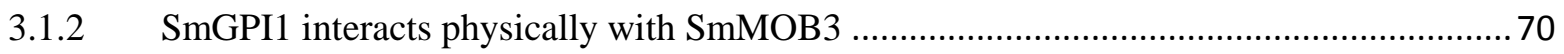

3.1.3 SmGPI1 binds to the cell wall and is partially secreted ................................................... 72

3.1.4 SmGPI1 localizes to the cell wall and mitochondria ..................................................... 75

3.1.5 Deletion of Smgpi1 restores fertility and hyphal fusion of sterile $\Delta$ Smmob3................... 78

3.1.6 $\Delta$ Smgpil forms more fruiting bodies that are small but normally shaped......................... 88

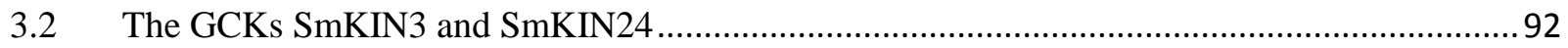

3.2.1 S. macrospora encodes two kinases similar to the mammalian STRIPAK-associated kinases STK24, STK25, MST4 and MINK1........................................................... 92

3.2.2 SmKIN3 interacts physically with PRO11 …............................................................ 100

3.2.3 Deletion of Smkin3 or Smkin24 impairs vegetative growth but only $\Delta$ Smkin24 is sterile 102

3.2.4 SmKIN3 and SmKIN24 localize to septa and influence septum formation......................107

3.2.5 $\Delta$ Smkin3 protoplasts recover significantly faster than wt protoplasts ............................. 109

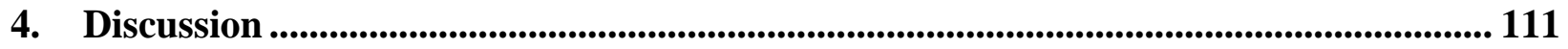

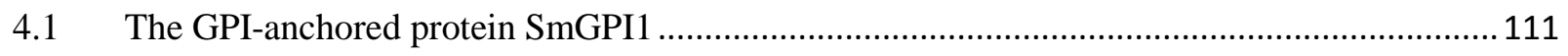

4.1.1 SmGPI1 is a GPI-anchored protein ...................................................................... 111

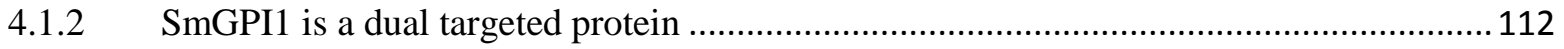

4.1.3 STRIPAK protein SmMOB3 interacts physically with SmGPI1.................................. 117

4.1.4 SmGPI1 is a positive regulator of fruiting-body number............................................. 118 
4.1.5 Smgpi1 deletion partially bypasses vegetative growth, hyphal fusion and fruiting-body development defects in $\Delta$ Smmob3 ............................................................................. 119

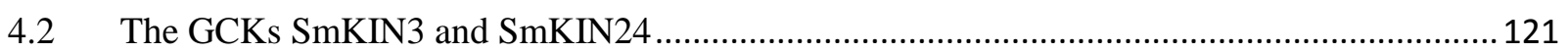

4.2.1 Are SmKIN3 and SmKIN24 STRIPAK-associated kinases? ....................................... 122

4.2.2 SmKIN3 and SmKIN24 affect growth velocity, sexual development and septum formation

4.2.3 The STRIPAK (-like) complex in S. macrospora ................................................... 126

5. References............................................................................................................................ 128

6. Acknowledgement........................................................... Fehler! Textmarke nicht definiert.

7. Curriculum vitae.............................................................. Fehler! Textmarke nicht definiert. 


\section{List of Tables}

Table 1. Mammalian STRIPAK components that associate with the complex or subcomplexes.............. 11

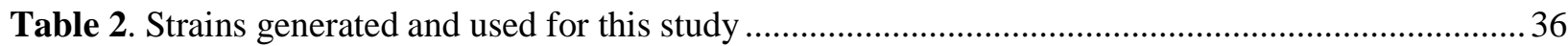

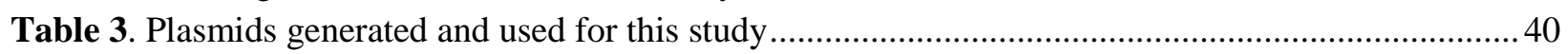

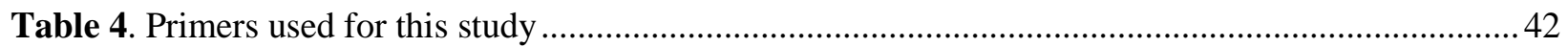

Table 5. Average growth value of $\Delta$ Smgpi1, complemented $\Delta$ Smgpi1, $\Delta$ Smmob3 and the double

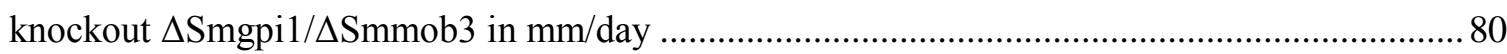

Table 6. BLASTP search of the human STRIPAK associated GC kinases against the S. macrospora

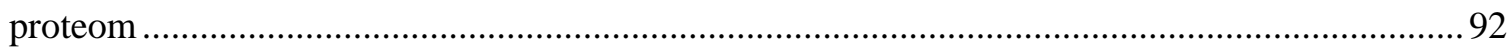




\section{List of Figures}

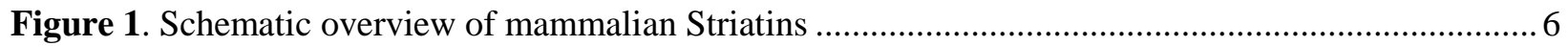

Figure 2. Mammalian STRIPAK complex(es) with its core components and additional proteins ............ 11

Figure 3. 3D structure of the heterotrimeric PP2A complex.............................................................. 14

Figure 4. Sequence alignment of human MOB1 with homologs from other species .............................. 15

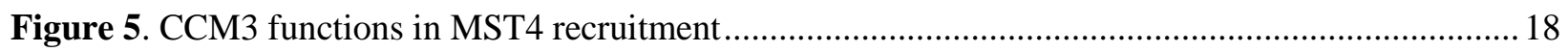

Figure 6. Aa sequence alignment, of mammalian GC III kinases..................................................... 20

Figure 7. Function of SIKE in IKK $\varepsilon$ and TBK1-mediated innate immune response................................26

Figure 8. Schematic overview about the STRIPAK core complex in signaling ...................................... 28

Figure 9. Schematic model of GPI-anchored protein precursors ....................................................... 31

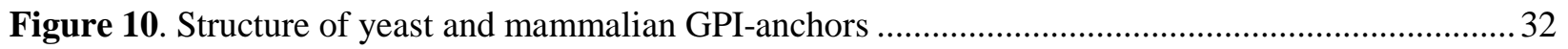

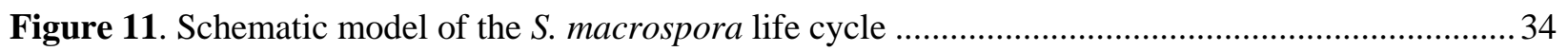

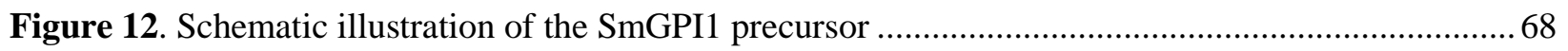

Figure 13. Multiple sequence alignment and aa identity of SmGPI1 with putatively homologue

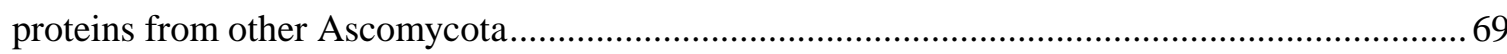

Figure 14. Yeast two-hybrid analysis of the interaction of SmGPI1 and SmMOB3 .............................. 70

Figure 15. Co-Immunoprecipitation of SmGPI1 and SmMOB3 with anti-FLAG and anti-eGFP

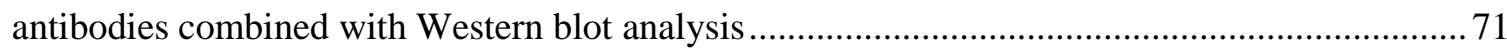

Figure 16. Western blot analysis of SmGPI1 after differential centrifugation of cellular components ..... 72

Figure 17. Schematic overview of SmGPI1 versions used for Western blot .......................................... 73

Figure 18. Western blot analysis of SmGPI1 aa 1-492 and aa 28-492 using cell-free supernatants

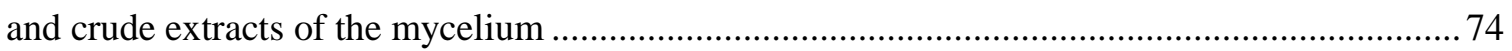

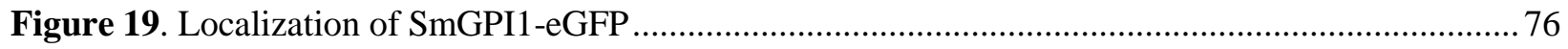

Figure 20. Localization of SmMOB3 in $\triangle$ Smgpi1 and SmGPI1 in $\Delta$ Smmob3 ..................................... 77

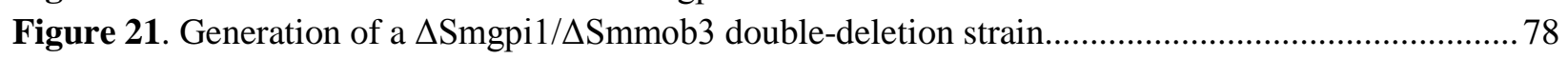

Figure 22. Verification of $\Delta$ Smgpi1/ $\Delta$ Smmob3 via PCR and Southern blot ........................................... 79

Figure 23. Sexual development of $\Delta$ Smgpi1, $\Delta$ Smmob3 and $\Delta$ Smgpi $1 / \Delta$ Smmob3 ................................ 81

Figure 24. Phenotypic analysis of $\Delta$ Smgpi1/ $\Delta$ Smmob3 complemented with full-length Smgpi1 or

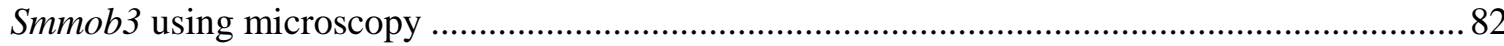

Figure 25. Generation of a $\Delta$ Smgpi1/ $\Delta$ pro11 double-deletion strain..................................................... 83

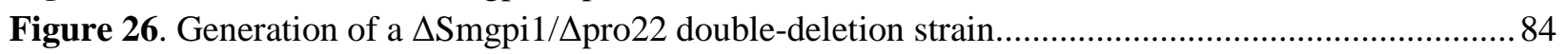

Figure 27. Generation of a $\Delta$ Smgpi1/ $\Delta$ pro45 double-deletion strain....................................................... 85

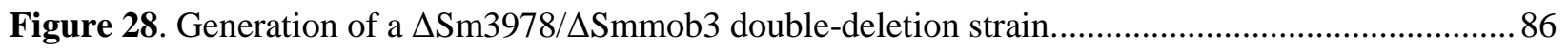

Figure 29. Deletion of Smgpi1 in a sterile $\Delta$ Smmob3 background restores hyphal fusion........................ 87

Figure 30. Number of fruiting bodies produced by $\Delta$ Smgpi1, $\Delta$ Smmob3 and $\Delta$ Smgpi $1 / \Delta$ Smmob3

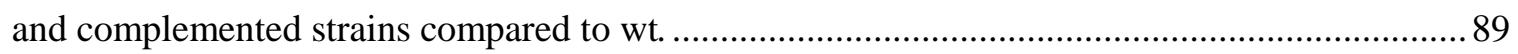

Figure 31. Deletion of Smgpi1 results in smaller mature fruiting bodies............................................... 90

Figure 32. Quantitative evaluation of perithecia size from wt, $\Delta$ Smgpil and $\Delta$ Smgpil expressing the full length Smgpi1, $\Delta$ Smgpi1/ $\Delta$ Smmob3 and wt expressing an additional copy of Smgpi1............... 91

Figure 33. Multiple sequence alignment and aa identity of mammalian kinases identified as STRIPAK members with putative homologues from Ascomycota 
Figure 34. RT-PCR analysis of Smkin3. (A) Schematic illustration of Smkin3 .................................... 95

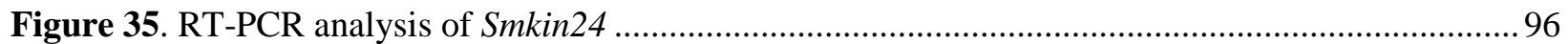

Figure 36. Alignment of aa sequences encoded by alternatively spliced Smkin24 transcripts ................. 97

Figure 37. Identity of aligned aa sequences of mammalian kinases identified as STRIPAK members with putative homologues from Ascomycota in pair-wise comparison. ........................ 98

Figure 38. Unrooted neighbor-joining tree of human GCKs MST4, STK24, STK25, MINK1

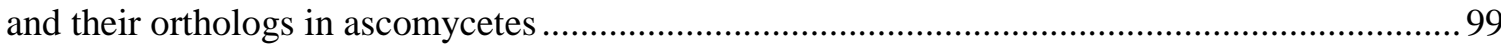

Figure 39. SmKIN3 and SmKIN24 interact physically with PRO11................................................. 101

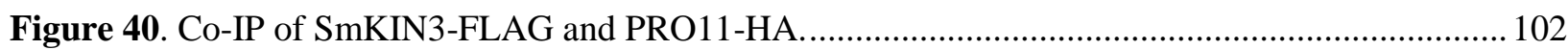

Figure 41. Generation of $\Delta$ Smkin3 and $\Delta$ Smkin24 deletion strains................................................ 103

Figure 42. Macroscopic and microscopic analysis of the sexual development of wt, $\Delta$ Smkin3, $\Delta$ Smkin24 and $\Delta$ Smkin3/ $\Delta$ Smkin24.

Figure 43. Macroscopic and microscopic analysis of the sexual development of wt, complemented $\Delta$ Smkin3, complemented $\Delta$ Smkin 24 and partially complemented $\Delta$ Smkin3/ $\Delta$ Smkin24.......... 105

Figure 44. Microscopic investigation of hyphal fusion in wt, $\Delta$ Smkin3, $\Delta$ Smkin24 and $\Delta$ Smkin3/ASmkin24.

Figure 45. Localization of SmKIN3-eGFP and SmKIN24-eGFP in S. macrospora.

Figure 46. Analysis of septal development in wt, $\Delta$ Smkin $3, \Delta$ Smkin 24 and $\Delta$ Smkin $3 / \Delta$ Smkin 24 and complemented mutants

Figure 47. Quantitative analysis of septal development in wt, $\Delta$ Smkin3, $\Delta$ Smkin 24 and $\Delta$ Smkin3/ $\Delta$ Smkin24 and complemented mutants.

Figure 48. Investigation of protoplast recovery and vegetative growth of $\Delta \operatorname{Smkin} 3, \Delta \operatorname{Smkin} 24$ and $\Delta$ Smkin $3 / \Delta$ Smkin24

Figure 49. SmGPI1 exhibits regions of disorder. 115

Figure 50. Dual targeting of SmGPI1 in S. macrospora

Figure 51. Schematic model of the genetic interplay between SmGPI1 and SmMOB3 and the STRIPAK complex in wt, single and double mutants 121

Figure 52. Schematic model for the interplay between STRIPAK and SIN in S. macrospora................ 124

Figure 53. Schematic model of STRIPAK complex in S. macrospora. 


\section{List of Abbreviations}

\begin{tabular}{|c|c|}
\hline aа & amino acids \\
\hline a. dest. & aqua destillata \\
\hline CMS & complete medium with saccharose \\
\hline CO-IP & co-immunoprecipitation \\
\hline bp & base pair \\
\hline BMM & biomalt maize medium \\
\hline BLAST & basic local alignment search tool \\
\hline cDNA & complementary DNA \\
\hline d & days \\
\hline DIC & differential interference contrast \\
\hline DsRED & encodes red fluorescence protein of Discosoma sp \\
\hline ER & endoplasmic reticulum \\
\hline eGFP & enhanced green fluorescence protein of Aequorea Victoria \\
\hline gDNA & genomic DNA \\
\hline GPI & glycosylphosphatidylinositol \\
\hline $\mathrm{kDa}$ & kilo Dalton \\
\hline ORF & open-reading frame \\
\hline PAGE & polyacrylamide gel electrophoresis \\
\hline PBS & phosphate buffered saline \\
\hline PCR & polymerase chain reaction \\
\hline RT-PCR & real-time PCR \\
\hline SD & selective dropout \\
\hline ssi & single spore isolate \\
\hline SWG & Sordaria Westergaards medium \\
\hline RT & Room temperatur \\
\hline $\mathrm{Y} 2 \mathrm{H}$ & yeast two-hybrid \\
\hline wt & wild type \\
\hline
\end{tabular}

Common abbreviations and units of measurement are not enlisted 


\section{Summary}

The mammalian Striatin-interacting phosphatase and kinase (STRIPAK) complex consist of many proteins, among them Striatin as scaffold, the putative kinase activator monopolar spindleone-binder 3 (MOB3), serine/threonine phosphatase PP2A subunits A and C, the Striatininteracting protein (STRIP)1 and STRIP2, sarcolemmal membrane-associated protein (SLMAP) and the germinal center kinases (GCK) MST4, STK24, STK25 and MINK1. In this study, we used the filamentous ascomycete Sordaria macrospora as model organism to analyze the role of the STRIPAK complex in fruiting-body development. S. macrospora is coprophytic fungus which solely undergoes a sexually lifecycle and does not require a mating partner for sexual reproduction.

In S. macrospora, the STRIPAK complex is required for fruiting-body development and hyphal fusion. Hyphal fusion is a process that results in mixed cell contents of involved cells without lysis. In filamentous ascomycetes, hyphal fusion occurs at different stages of vegetative growth and sexual reproduction.

The STRIPAK complex in S. macrospora contains homologs to mammalian Striatin (PRO11), MOB3 (SmMOB3), subunits A and C of PP2A (SmPP2AA and C), STRIP1/2 (PRO22) and SLMAP (PRO45). However, fungal STRIPAK-associated kinases have not been characterized to date.

This study is divided into two parts, one comprises the characterization of SmGPI1, a GPIanchored protein, identified as interaction partner of SmMOB3 in cross-species microarrays, and the other part is about identification of potential STRIPAK-associated kinases. Interaction between STRIPAK-associated SmMOB3 and SmGPI1 was successfully verified by coImmunoprecipitation (co-IP) and yeast two-hybrid (Y2H) using S. macrospora cDNA. Deletion of Smgpi1 was the next step to investigate its impact on fruiting-body development; in contrast to $\Delta$ pro11, $\Delta$ pro22, $\Delta$ SmMOB3 and $\Delta$ pro45, $\Delta$ Smgpil underwent hyphal fusion and was fertile, but generated more fruiting bodies, which were smaller but normal in shape compared to wt. Interestingly deletion of Smgpi1 in a sterile $\Delta$ Smmob3 deletion background restored the phenotypes caused by $S m m o b 3$ deletion. As already mentioned, $\Delta$ Smmob3 is sterile and not capable of hyphal fusion. In contrast, the double-deletion strain $\Delta$ Smgpi $1 / \Delta$ Smmob3 is fertile and underwent hyphal fusion. This effect was Smmob3 specific and did not occur in other 
STRIPAK-specific double-deletion strains, e.g. $\Delta$ Smgpi1/ $\Delta$ pro11, $\Delta$ Smgpi1/ $/ \Delta$ pro22 and $\Delta$ Smgpi $1 / \Delta$ pro45. Moreover, fluorescence microscopy and differential centrifugation of SmGPI1 revealed a dual targeting; SmGPI1 localizes at the cell wall and the mitochondria.

Regarding the identification of STRIPAK-associated kinases in S. macrospora, two kinases were identified to be homologous to the mammalian STRIPAK-associated kinases MST4, STK24, STK25 and MINK1 by BLASTP search and were named SmKIN3 and SmKIN24. Phylogenetic analysis revealed a conservation of these kinases among ascomycetes. Interaction of SmKIN3 and SmKIN24 with S. macrospora Striatin homolog PRO11 was shown via Y2H and for SmKIN3 and PRO11 also by means of co-IP. Fluorescence microscopy of SmKIN3 and SmKIN24 revealed localization to the septa, whereas SmKIN3 localizes at the outer part of the septum and SmKIN24 to the septal pore.

Deletion of Smkin3 or Smkin24 to analyze their impact on fruiting-body development showed only $\Delta$ Smkin 24 to be sterile. However, $\Delta$ Smkin3 and $\Delta$ Smkin24 were reduced in vegetative growth and exhibited impaired septa formation; whereas $\Delta$ Smkin3 displayed greater distances between adjacent septa compared to wt, deletion of Smkin24 resulted in numerous closelypacked septal bundles of abnormal shape. Although phenotypically distinct, both kinases appear to function independently because the double-knockout strain $\Delta$ Smkin $3 / \Delta \operatorname{Smkin} 24$ displayed the combined phenotypes of each single-deletion strain. Moreover, we discovered that protoplasts harboring the $\Delta$ Smkin3 deletion background recover faster than protoplasts obtained from wt. Based on the results of this study and findings in N. crassa that homologs to SmKIN3 and SmKIN24 are implicated in septation initiation network (SIN) we assume STRIPAK to function aside from sexual development and hyphal fusion, also in regulation of fruiting-body number via SmMOB3-SmGPI1 interaction and suggest a crosstalk between SIN and STRIPAK in S. macrospora. 


\section{Zusammenfassung}

Der STRIPAK (für engl. „, Striatin-interacting phosphatase a nnd kinase”)-Komplex in Säugetieren umfasst unter anderem die Proteine Striatin als Gerüsteinheit, Phosphatase 2A, Untereinheit A und C, STRIP (für engl „ㅌtriatin-interacting protein“) 1 und 2, den putativen Kinaseaktivator MOB3 und die GC (für engl. „germinal center“) Kinasen MST4, STK24, STK25 und MINK1, sowie SLMAP (für engl. „șarcolemmal membrane-ạssociated protein“). Im Rahmen dieser Arbeit wird S. macrospora als Modellorganismus für die Analyse der Funktion des STRIPAK Komplexes auf die Entwicklung von Fruchtkörpern verwendet. S. macrospora ist ein koprophiler selbstfertiler Schlauchpilz (Ascomyzet), der lediglich einen sexuellen Lebenszyklus aufweist. Der STRIPAK-Komplex in S. macrospora ist beteiligt an verschiedenen Prozessen, darunter die sexuelle Entwicklung und die Hyphenfusion. Die Hyphenfusion beschreibt die Verbindung von zwei Zellen, ohne dabei zu lysieren. In S. macrospora wurden bisher Homologe zu den in Säugern identifizierten Proteinen Striatin (PRO11), MOB3 (SmMOB3), PP2AA und C (SmPP2AA und C), STRIP1/2 (PRO22) und SLMAP (PRO45) identifiziert, jedoch wurden bisher noch keine beteiligten Kinasen identifiziert.

Diese Arbeit befasst sich mit zwei Hauptthemen: Der Charakterisierung von SmGPI1, ein GPIgeankertes Protein, welches als Interaktionspartner von SmMOB3 identifiziert wurde und der Identifizierung und Charakterisierung potentieller STRIPAK-Kinasen.

Im Rahmen dieser Arbeit konnte die Interaktion zwischen SmGPI1 und SmMOB3 erfolgreich mittels Y2H und co-IP bestätigt werden. Zur Untersuchung des Einflusses von Smgpi1 auf die Fruchtkörperentwicklung, wurde das Gen deletiert. Im Unterschied zu Stämmen, die aus der Deletion von Genen, welche für Proteine des STRIPAK-Komplexes codieren, hervorgingen, ist $\Delta$ Smgpil fertil und fähig zur Hyphenfusion. Im Vergleich zum Wildtyp, sind die Fruchtkörper von $\Delta$ Smgpil zwar kleiner, aber normal geformt.

Interessanterweise, führt die Deletion von Smgpi1 in der sterilen Deletionsmutante $\Delta \mathrm{Smmob} 3 \mathrm{zu}$ einer Unterdrückung des $\Delta$ Smmob3 Phänotyps. Während $\Delta$ Smmob3 steril ist und keine Hyphenfusion zeigt, ist $\Delta$ Smmob3/ $\Delta$ Smgpi1 fertil und befähigt zur Hyphenfusion. Dieser Effekt ist spezifisch und tritt nur in der Deletionsmutante $\Delta$ Smmob3/ $\Delta$ Smgpil auf.

Des Weiteren wurde durch Fluoreszenzmikroskopie und differenzieller Zentrifugation gezeigt, daß SmGPI1 dual lokalisiert ist: SmGPI1 wurde sowohl an der Zellwand, als auch in den Mitochondrien gefunden. 
Bezüglich der Identifikation von STRIPAK-Kinasen, wurden zwei potentielle Homologe zu den in Säugern identifizierten STRIPAK-Kinasen mittels BLASTP-Suche identifiziert, namentlich SmKIN3 und SmKIN24. Die Interaktion von SmKIN3, SmKIN24 und dem S. macrospora Striatin-Homolog PRO11 wurde mittels Y2H bestätigt, die Interaktion von SmKIN3 mit PRO11 zusätzlich auch durch co-IP. Fluoreszenzmikroskopie der eGFP markierten Proteine SmKIN3 und SmKIN24 ergab eine Lokalisierung dieser an den Septen. Die Deletion der beiden Gene Smkin3 und Smkin24 zeigte, daß lediglich SmKIN24 an der Fruchtkörperentwicklung beteiligt ist, was sich in Sterilität der Deletionsmutante $\Delta$ Smkin24 zeigte. Der Stamm $\Delta$ Smkin3 war weiterhin fertil. Interessanterweise zeigten beide Stämme einen Defekt in der Entwicklung von Septen und dem vegetativen Wachstum. Während die Abstände zwischen nebeneinanderliegenden Septen in $\Delta$ Smkin3 im Verhältnis zum Wildtyp vergrößert waren, führte die Deletion von Smkin24 zu vielen, dicht gepackten und deformierten Septen. Dennoch scheinen beide Proteine unabhängig zu fungieren, da der Doppel-Knockout $\Delta \operatorname{Smkin3/\Delta Smkin24}$ die Phänotypen beider Einzelnockouts zeigt. Des Weiteren zeigten Protoplasten, welche den $\Delta$ Smkin3 Deletionshintergrund besaßen, einen deutlich erhöhten Regenerationseffekt.

Basierend auf den Ergebnissen dieser Studie kann dem STRIPAK-Komplex in S. macrospora neben der sexuellen Entwicklung und der Hyphenfusion auch eine Funktion in der Regulierung der Fruchtkörperanzahl zugewiesen werden. Zusätzlich vermuten wir eine Verbindung zwischen STRIPAK-Komplex und des Netzwerkes, welches die Septierung einleitet (SIN, für engl. „septation initiation network“). 


\section{Introduction}

Organisms underlie many environmental changes and pressures, such as different salt concentrations, changing nutrient conditions, pheromones, oxidative stress or $\mathrm{pH}$ values (Maller, 2003, Martindale \& Holbrook, 2002). Thus, it is necessary to respond appropriately to the incoming stimuli and to generate respective responses. On molecular level, the response to different stimuli is facilitated by signaling pathways. To date, many signal transduction pathways have been identified, partially connected to each other. Normally, incoming signals are detected by receptors on the cell surface, which modulate the signal and transmit it to the cell lumen. These signals can be enhanced and transduced by many modifications, for example phosphorylation or protein cleavage (Li \& Hristova, 2006, Lieber et al., 1993). In general, signal transduction ends in changed translation pattern of genes, needed for the appropriate response to the stimulus (Lalli \& Sassone-Corsi, 1994). Dysfunctions in signal transduction and responses to environmental changes can cause severe diseases such as cancer (Wu et al., 2010).

In the recent years, Striatins have been identified to be regulators of various differentiation processes and thus, it might have a key role in these processes (Benoist et al., 2006).

\subsection{Striatins in mammals}

In mammals, the group of Striatins comprises the three proteins Striatin, $\mathrm{SG}_{2} \mathrm{NA}$ and Zinedin (Figure 1). These proteins are highly similar in their protein domain structure and localization. Functional and structural homologs were discovered in many other organisms like Drosophila melanogaster, Schizosaccharomyces pombe, Neurospora crassa, Saccharomyces cerevisiae and Sordaria macrospora (Bloemendal et al., 2012, Lisa-Santamaria et al., 2012, Pöggeler \& Kück, 2004, Simonin et al., 2010, Tanabe et al., 2001). Mammalian Striatins are characterized by a caveolin-binding side, a $\mathrm{Ca}^{2+}$-Calmodulin binding side, a coiled-coil domain and tryptophanaspartate (WD) repeat (hereafter WD40) repeats (Benoist et al., 2006).

Caveolins are small integral membrane proteins and the main component of the caveolae; cholesterol-rich, sack shaped, invaginated lipid rafts in the plasma membrane of cells (Parton \& Simons, 2007). Caveolins interact with many signaling proteins. This is facilitated by an about 20 aa comprising motif at their N-terminus ( $\mathrm{Li}$ et al., 1996). The consensus sequence of 
caveolin-interaction motifs as present in Striatins is $\Phi \mathrm{XXXXФХХФ,} \mathrm{where} \Phi$ represents an aromatic aa and X represents random aa (Couet et al., 1997, Benoist et al., 2006).

Calmodulin-binding motifs are protein domains necessary for interaction of proteins with Calmodulin. Calcium-modulated protein (Calmodulin), is a calcium-binding messenger protein expressed in all eukaryotic cells. Interaction with its target proteins is modulated by calcium-ion binding and thus, Calmodulin (CaM) converts calcium concentrations into further signals (Chin \& Means, 2000, Stevens, 1983). CaM is highly conserved and consists of approximately 148 aa with a molecular weight of $16.7 \mathrm{kDa}$. It contains four motifs for $\mathrm{Ca}^{2+}$-ion binding. The tertiary structure shows two globular domains representing the N- and C-terminal domain, separated by a flexible linker region (Chin \& Means, 2000)

Striatin $\mathrm{N}||$\begin{tabular}{|l|l|l|l|}
\hline & $\mathrm{cc}$ & & \\
\hline
\end{tabular}
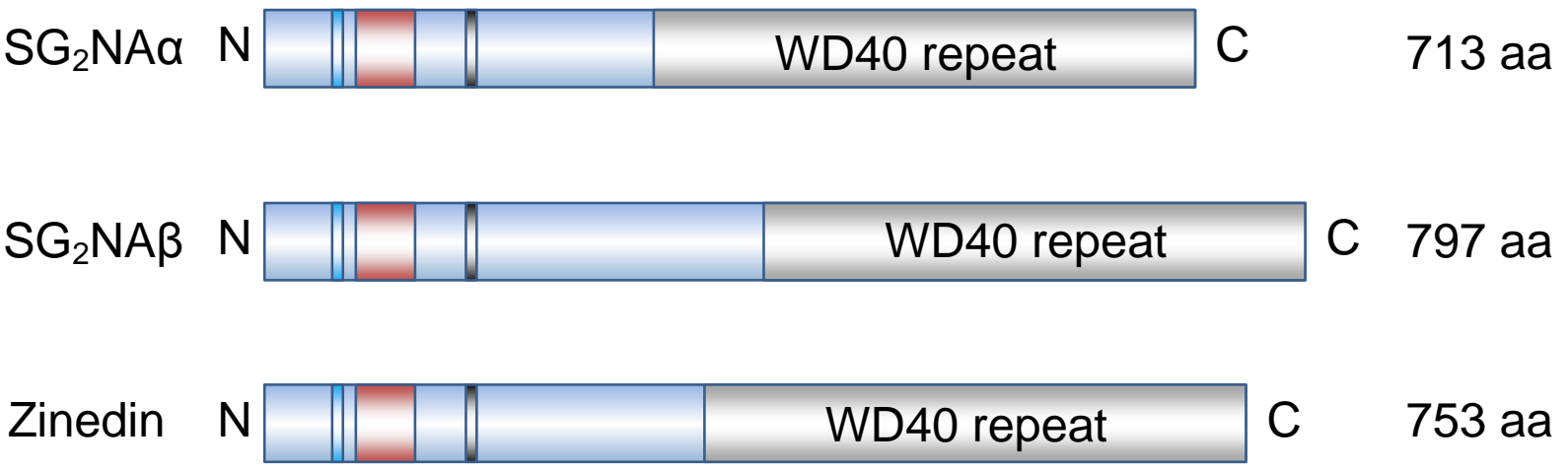

Figure 1. Schematic overview of mammalian Striatins; shown are the structural domains of Striatin, $\mathrm{SG}_{2} \mathrm{NA}$ isoform $\alpha$ and $\beta$, and Zinedin. $\mathrm{cv}=$ caveolin binding domain, $\mathrm{cc}=$ coiled-coil domain, $\mathrm{cm}=\mathrm{Ca}^{2+}$ calmodulin binding domain, $\mathrm{N}=\mathrm{N}$-terminus, $\mathrm{C}=\mathrm{C}$-terminus. Domains shown for $\mathrm{SG}_{2} \mathrm{NA}$ and Zinedin are predicted and not experimentally proved. Aa in total is given at the end of each protein (according to Hwang \& Pallas (2014)).

Coiled-coil domains are motifs of 2-7 alpha helices that are coiled together like rope strands. The common types are dimers and trimers (Liu et al., 2006). Coiled-coil motifs facilitate oligomerization of many proteins (Burkhard et al., 2001). The consensus sequence of coiled-coil motifs is the repeated pattern HxxHCxC, with $\mathrm{H}=$ hydrophobic aa and $\mathrm{C}=$ charged aa, referred 
Introduction

to as heptad repeat (Mason \& Arndt, 2004). For Striatin, oligomerization was shown to be crucial for some interactions with other proteins (Chen et al., 2012, Gaillard et al., 2006).

The WD40 domain is formed by 4 to 16 structurally conserved WD40 repeats (Li \& Roberts, 2001, Smith et al., 1999). WD40 repeats consist of 44-60 aa, containing tryptophan-aspartic acid (W-D) dipeptides at the C-terminus and a glycine-histidine pair at 11-24 aa positions from their N-terminus (Neer et al., 1994, van der Voorn \& Ploegh, 1992). The WD40 domain forms a propeller-like structure of interlocked beta sheets that serves for protein complex assembly (Hwang \& Pallas, 2014, Li \& Roberts, 2001).

\subsubsection{Striatin}

Striatin was first identified in rat brain and named after the striatum, a part of the cerebrum, where it was found most abundantly (Castets et al., 1996). In neural tissues, Striatin was also identified throughout the central and peripheral nervous system but mostly in the striatum and motoneurons. Moreover, Striatin is also expressed in many other tissues, among them fibroblasts, lymphocytes, lung, liver, kidney, skeletal and cardiac muscles (Benoist et al., 2006, Castets et al., 1996, Castets et al., 2000, Moqrich et al., 1998, Moreno et al., 2000). Striatin full-length protein comprises 780 aa and consists of the 4 characteristic domains for interaction; a caveolinbinding motif, a coiled-coil region, a CaM-binding motif and a WD40 repeat domain (Figure 1). Until today only little is known about the function of Striatin-caveolin interaction. Since Striatin was shown to interact with Caveolin-1 (CAV-1), which may act as a scaffolding protein within caveolar membranes, this interaction was hypothesized to facilitate Striatin localization to Caveolin-1 rich domains in dendric spines (Gaillard et al., 2001).

The $\mathrm{Ca}^{2+}-\mathrm{CaM}$ binding domain of Striatin comprises aa 149-166 and is crucial for Striatin-CaM interaction in a $\mathrm{Ca}^{2+}$-dependent manner. Until today, it is still unknown, how Striatins function in $\mathrm{Ca}^{2+}$ signaling. One hypothesis is that Striatin functions as $\mathrm{Ca}^{2+}$ sensor that reacts to changing $\mathrm{Ca}^{2+}$ concentrations (Benoist et al., 2006). This is among others based on results showing that physiologically relevant calcium concentrations increase presence of cytosolically located Striatin as well as findings, that Striatin distribution in cytosolic, detergent soluble and insoluble fractions of brain tissue depends on calcium presence or absence during lysis (Bartoli et al., 1998, Gaillard et al., 2001). Furthermore, the CaM-binding motif of Striatin appears to function 
Introduction

in protein-protein interaction of Striatin; deletion of its CaM-binding motif enhances interaction of Striatin with germinal center kinases (GCK) mammalian STE20-like protein kinase 4 (MST4) and serine/threonine-protein kinase (STK) 24. By this, $\mathrm{Ca}^{2+}$-CaM binding could regulate MST4 and STK24 binding to Striatin by modifying the subcellular localization of Striatin. However, interaction with other tested proteins was not affected (Gordon et al., 2011).

Additionally, it was shown that Striatin hetero-oligomerizes through its coiled-coil domain with Zinedin, but also homo-oligomerizes (Gaillard et al., 2006). The coiled-coil domain of Striatin comprises aa 64-120, but with the possibility that even parts of the caveolin binding domain belongs to it. Moreover, a trigger sequence necessary for successful coiled-coil interaction is assumed to be located at the N-terminus of Striatin (Gordon et al., 2011).

Striatin contains a WD40 domain composed of 6 or 7 WD40 repeats. It was shown that deletion of this region abolishes interaction of Striatin with other proteins, among them the kinase activator monopolar spindle-one-binder 3 (MOB3)/phocein (Baillat et al., 2001, Moreno et al., 2001).

Striatin is absent from axons but highly concentrated in dendric spines. Based on the $\mathrm{Ca}^{2+}-\mathrm{CaM}$ binding motif, it is likely regulated by $\mathrm{Ca}^{2+}$-dependent signaling in postsynaptic neurons (Castets et al., 1996).

\subsection{2 $\quad \mathrm{SG}_{2} \mathrm{NA}$}

$\mathrm{SG}_{2} \mathrm{NA}$ was first reported to localize to the nucleus. It is named after its expression levels which have their maximum during the $S$ and the $G_{2}$ phase of the cell cycle (Muro et al., 1995). Later it was shown to localize predominantly to the cytosol or membranes. $\mathrm{SG}_{2} \mathrm{NA}$ displays the highest expression in cerebellum and cortex where it, similar to Striatin, localizes to somato-dendritic spines with high concentration in dendric spines. However, it is also present in other tissues (Castets et al., 2000, Moreno et al., 2001). As shown in Figure 1, $\mathrm{SG}_{2} \mathrm{NA}$ protein domains resemble Striatin. Similarly, it consists of a caveolin-binding domain from aa 71-79, a $\mathrm{Ca}^{2+}-\mathrm{CaM}$ binding domain from aa 166-183, a coiled-coil domain ranging from aa 77-136 and a WD40 domain of 6 WD40 repeats. $\mathrm{SG}_{2} \mathrm{NA}$ was shown bind $\mathrm{CaM}$ in a $\mathrm{Ca}^{2+}$-dependent manner. This Striatin variant homo-oligomerizes and hetero-oligomerizes with Zinedin and Striatin by their coiled-coil domains. The coiled-coil domain of $\mathrm{SG}_{2} \mathrm{NA}$ was shown to be necessary but not 
Introduction

sufficient to target the protein to dendric spines (Gaillard et al., 2006). Two major isoforms of $\mathrm{SG}_{2} \mathrm{NA}$ are known; $\mathrm{SG}_{2} \mathrm{NA} \alpha$ with 713 aa and $\mathrm{SG}_{2} \mathrm{NA} \beta$ with a length of 797 aa (Figure 1) (Benoist et al., 2006). However, minor isoforms do also exist; $\mathrm{SG}_{2} \mathrm{NA} \gamma$ was identified previously in rat brain tissue. It lacks all but one WD40 repeats and localizes to the nucleus. $\mathrm{SG}_{2} \mathrm{NA} \gamma$ was shown to organize an estrogen-inducible complex of protein phosphatase $2 \mathrm{~A}$ (PP2A) and estrogen receptor $\alpha(\mathrm{ER} \alpha)$ (Tan et al., 2008). Moreover, $\mathrm{SG}_{2} \mathrm{NA}$ exhibits transcriptional activation activity (Zhu et al., 2001). Among Striatins, $\mathrm{SG}_{2} \mathrm{NA}$ is the most conserved one (Tanti et al., 2014).

\subsubsection{Zinedin}

Zinedin was first identified by search for Striatin homologs (Castets et al., 2000). The canonical full-length isoform comprises 753 aa. Similar to Striatin and $\mathrm{SG}_{2} \mathrm{NA}$, Zinedin exhibits the four characteristic domains for protein interaction (Figure 1). Zinedins caveolin-binding domain comprises aa 71-79, its $\mathrm{Ca}^{2+}$-CaM binding domain aa 165-182. Zinedin hetero-oligomerizes with $\mathrm{SG}_{2} \mathrm{NA}$ and binds similar to Striatin and $\mathrm{SG}_{2} \mathrm{NA} \mathrm{CaM}$ in a $\mathrm{Ca}^{2+}$-dependent manner. Zinedin exhibits 7 WD40 repeats ranging from aa 436 to 752 . It is most abundantly expressed in the hippocampus and localizes to somato-dendritic spines with high concentration in dendric spines but is also found in various other tissues (Benoist et al., 2008, Gaillard et al., 2006, Gordon et al., 2011). Within the cell, Zinedin localizes cytosolically (Blondeau et al., 2003, Castets et al., 2000).

\subsection{Striatin homologs in other eukaryotes}

Additionally to the mammalian Striatins, homologs have been identified in other animals and lower eukaryotes, among them the isoforms $\mathrm{SG}_{2} \mathrm{NA \alpha}$ and $\mathrm{SG}_{2} \mathrm{NA} \alpha+$ from goldfish, D. melanogaster "connector of kinase to AP-1" (CKA), Caenorhabditis elegans "CKA and Striatin homolog family member" (CASH-1), S. cerevisiae "Factor arrest protein 8” (FAR8), S. pombe FAR8/CSC3p, N. crassa "hyphal anastomosis mutant 3" (HAM-3), S. macrospora PRO11 (protoperithecia mutant 11), Fusarium graminearum "Fusarium verticillioides Striatin ortholog 1” (FSR1) and Aspergillus nidulans “A nidulans Striatin” 
Introduction

(STRA). All these proteins harbor characteristic domains as described for Striatins (Bloemendal et al., 2012, Chen et al., 2002, Ma et al., 2009, Pöggeler \& Kück, 2004, Simonin et al., 2010, Shim et al., 2006).

Deletion of genes encoding for Striatin homologs have a high impact on developmental processes; deletion of pro11 in S. macrospora, fsr1 in F. graminearum, strA in A. nidulans and ham-3 in N. crassa lead to sterility. Moreover, the deletion strains S. macrospora $\Delta$ pro11 and N. crassa $\Delta$ ham-3 and were not capable of hyphal fusion (Bernhards \& Pöggeler, 2011, Bloemendal et al., 2012, Shim et al., 2006, Simonin et al., 2010, Wang et al., 2010). Furthermore, decreases deletion of $f s r 1$ in $F$. graminearum its virulence. Cell fusion, which is also named hyphal fusion in filamentous fungi, occurs in organisms from eukaryotic microbes to multicellular plants and animals and results in mixed cell contents of involved cells without lysis. In filamentous ascomycetes, hyphal fusion occurs at different stages of vegetative growth and sexual reproduction (Rech et al., 2007, Fleißner et al., 2008, Read et al., 2010, Bloemendal et al., 2012). This emphasizes the importance of Striatins (Hwang \& Pallas, 2014). Interestingly, sterility of the $S$. macrospora pro11 mutant can be complemented with Striatin cDNA from mouse, showing an evolutionary conservation of Striatins function in signaling (Pöggeler \& Kück, 2004). Recent studies revealed that Striatins have evolved from prokaryotic counterparts, but acquired domains exclusive for metazoans. Within this process, $\mathrm{SG}_{2} \mathrm{NA}$ might be the earliest evolved Striatin (Tanti et al., 2014).

\subsection{Striatin-family complexes}

Striatins have been shown to interact with a broad number of proteins, among them $\mathrm{Ca}^{2+}$-CaM, CAV-1 and monopolar spindle-one-binder 3/phocein (MOB3) (Baillat et al., 2001, Castets et al., 2000, Gaillard et al., 2006, Moreno et al., 2001). Moreover, based on their WD40 domain, Striatins are assumed to function as a scaffolding unit for protein interactions (Moreno et al., 2000, Pöggeler \& Kück, 2004). Striatin-family members have been shown to interact with the structural (A) and catalytic (C) subunit of PP2A, germinal center kinases (GCK) and other proteins. This led to the name Striatin-interacting phosphatase and kinase (STRIPAK) for protein complexes consisting of Striatins as scaffolding unit, PP2A and kinases (Goudreault et al., 2009, Hyodo et al., 2012). Moreno et al. (2001) postulated regarding the PP2A-Striatin interaction that 
Striatin functions as novel B"', family of PP2AB-type regulatory subunits by interaction with the PP2A heterodimer. The mammalian STRIPAK complex is a multi-protein complex. Its core components are Striatin as scaffold, PP2A subunit A and C, MOB3, the Striatin-interacting proteins (STRIP)1 and STRIP2 (formerly named Fam40a and Fam40b), cerebral cavernous malformation 3 (CCM3; also called programmed cell death 10, PDCD10) and the mammalian sterile 20-like (MST) kinase, subclass GCK III, MST4,_serine/threonine-protein kinase (STK) 24 and STK25 (Goudreault et al., 2009) (Table 1).

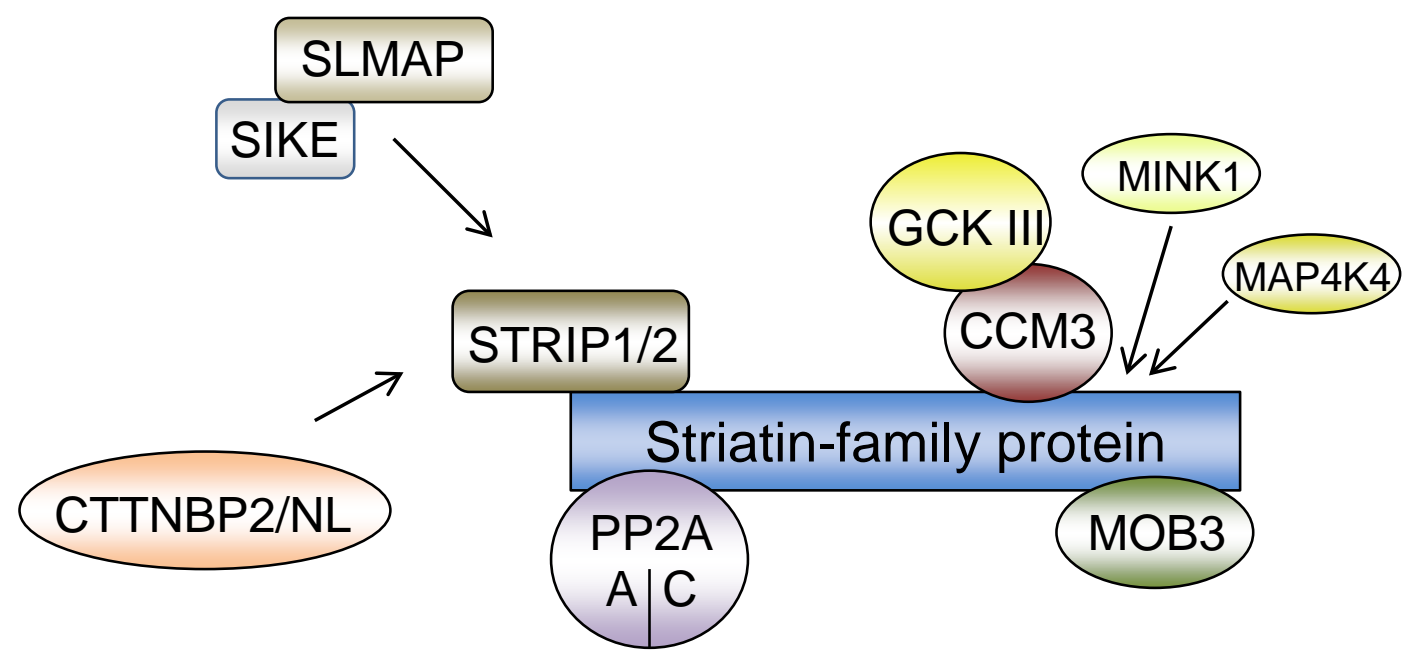

Figure 2. Mammalian STRIPAK complex(es) with its core components and additional proteins. The Striatin-family proteins comprise Striatin, $\mathrm{SG}_{2} \mathrm{NA}$ or Zinedin. Core components are the PP2A heterodimer with subunit A and C, MOB3, STRIP1 and STRIP2 as well as kinases of the GC III class, MST4, STK24 and STK25 recruited by CCM3. It is still unknown weather Mishappen-like kinase $\underline{1}$ (MINK1) and Mitogen-ạctivated protein kinase kinase kinase kinase $\underline{4}$ (MAP4K4) are also recruited by CCM3. Some proteins bind mutually exclusive to Striatin-family members (e.g. CTTNBP2/NL, SLMAP and SIKE) resulting in STRIPAK subcomplexes. These interactions are indicated by arrows. The simultaneous interaction of MINK1, MAP4K4 and GCK III has not been proven and thus is assumed to be bind in a mutually exclusive manner. The interaction partners of Striatins are described in the text (according to Hwang \& Pallas (2014)).

Table 1. Mammalian STRIPAK components that associate with the complex or subcomplexes

\begin{tabular}{l|l|l}
\hline \multicolumn{1}{c}{ Protein name } & \multicolumn{1}{c}{ Full name/description } & \multicolumn{1}{c}{ Reference } \\
\hline $\begin{array}{l}\text { Striatin, } \\
\mathrm{SG}_{2} \mathrm{NA}, \\
\text { Zinedin }\end{array}$ & $\begin{array}{l}\text { Striatin, putative regulatory subunit B', of } \\
\text { protein phosphatase 2A }\end{array}$ & (Moreno et al., 2000) \\
\hline PP2AA, PP2AC & $\begin{array}{l}\text { Structural (A) and catalytic (C) subunits of } \\
\text { protein phosphatase 2A }\end{array}$ & (Moreno et al. 2000) \\
\hline
\end{tabular}


Introduction

\begin{tabular}{|c|c|c|}
\hline MOB3 & 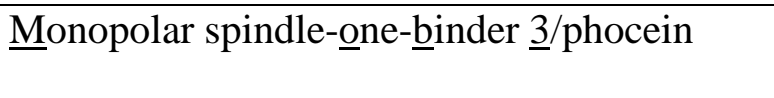 & $\begin{array}{l}\text { (Baillat et al., 2001, Moreno et } \\
\text { al., 2001) }\end{array}$ \\
\hline CCM3 & $\begin{array}{l}\text { Cerebral } \underline{\text { cavernous }} \text { malformation } \underline{3} / \\
\text { programmed cell death } 10\end{array}$ & \multirow[t]{9}{*}{ (Goudreault et al., 2009) } \\
\hline MST4 & $\begin{array}{l}\text { Mammalian sterile } 20 \text {-like kinase } \underline{4} \text {, subclass } \\
\text { of GC III kinases }\end{array}$ & \\
\hline STK24 & 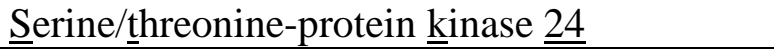 & \\
\hline STK25 & 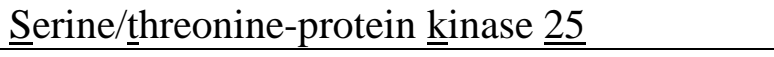 & \\
\hline STRIP1/2 & $\begin{array}{l}\text { Striatin-interacting protein } \underline{1} \text { and } \underline{2} \text {, formerly } \\
\text { Fam40a and Fam40b }\end{array}$ & \\
\hline SLMAP & Sarcolemmal membrane-a associated protein & \\
\hline CTTNBP2/NL & $\begin{array}{l}\text { Cortactin-binding protein } \underline{2} \text { /cortactin-binding } \\
\text { protein } \underline{2}, \underline{\text { N-terminal-like }}\end{array}$ & \\
\hline SIKE & 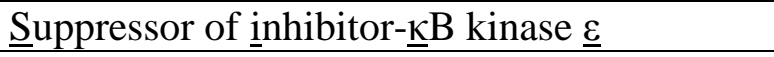 & \\
\hline FGFR1OP2 & $\begin{array}{l}\text { Fibroblast growth factor receptor } \underline{1} \text { oncogene } \\
\text { partner } \underline{2}\end{array}$ & \\
\hline MINK1 & Misshapen-like kinase $\underline{1}$ & (Hyodo et al., 2012) \\
\hline
\end{tabular}

As shown in Figure 2, several interaction partners bind mutually exclusive to the Striatin core complex. Goudreault et al. (2009) showed that either a cortactin-binding protein 2 family members (CTTNBP2 or CTTNBNL) or sarcolemmal membrane-asssociated protein (SLMAP) with suppressor of inhibitor-ㅌB kinase $\underline{\varepsilon}$ (SIKE) bind simultaneously to Striatin. This binding behavior results in STRIPAK subcomplexes, which are similar in its core components but differ in its additional interaction partners (Hwang \& Pallas, 2014).

Moreover, STRIPAK-like complexes have been identified. These complexes contain at least Striatin as a scaffolding unit and the PP2A subunits PP2AA and PP2AC; however, in most cases, the presence of kinases has not been demonstrated. STRIPAK-like complexes have been identified in mammals (see 1.3.2), D. melanogaster, yeast and filamentous ascomycetes. (Frost et al., 2012, Simonin et al., 2010, Xiang et al., 2002, Ma et al., 2009, Chen et al., 2002, Ribeiro et al., 2010, Singh et al., 2011, Hwang \& Pallas, 2014, Bloemendal et al., 2012, Dettmann et al., 2013).

The D. melanogaster STRIPAK complex contains homologs of Striatin, PP2A, MOB3, STRIP, fibroblast growth factor receptor $\underline{1}$ oncogene partner $\underline{2}$ (FGR10P2)/SIKE and CCM3. Additionally, the GC kinase Hippo (HPO) was identified as part of this complex. HPO is a homolog to mammalian MST1 and MST2, which are not STRIPAK-associated in mammals (Ribeiro et al., 2010). However, STRIPAK-like complexes have also been identified in $D$. 
melanogaster, involved in processes, such as activation of mitogen-activated protein kinases in the c-Jun N-terminal kinase (JNK) pathway (Ribeiro et al., 2010, Chen et al., 2002). JNKs are mitogen-activated protein kinase that respond to stress stimuli (Ip \& Davis, 1998).

The STRIPAK-like complex in S. cerevisiae is named Factor arrest (FAR) complex and consists of proteins which are homologous to Striatin, SLMAP, STRIP and PP2As subunits A and C (Frost et al., 2012, Lisa-Santamaria et al., 2012). S. cerevisiae does not encode a MOB3 homolog.

In filamentous fungi, such as $N$. crassa and S. macrospora, STRIPAK-like complexes without homologs of mammalian kinases have been identified. These complexes contain proteins homologous to Striatin, PP2AA, PP2AC STRIP, SLMAP and MOB3 (Bloemendal et al., 2012, Dettmann et al., 2013, Nordzieke et al., 2014, Simonin et al., 2010, Xiang et al., 2002).

\subsubsection{Function of STRIPAK components}

Numerous proteins have been identified to be members of the mammalian STRIPAK complex or to interact with its subunits. Proteins that have been identified as part of the complex or subcomplexes are enlisted in Table 1 and explained in detail in this section.

\subsubsection{Protein phosphatase 2A}

Mammalian PP2A is a herotrimeric serine/threonine phosphatase that contains a $65 \mathrm{kDa}$ scaffolding A subunit, a $36 \mathrm{kDa}$ catalytic C subunit and a regulatory B (separated in B, B', B', and B',') subunit (Dettmann et al., 2013). Subunit A of PP2A contains 15 tandem repeats of a conserved 39-residue sequence called HEAT (ㅂuntingtin, elongation factor 3 (EF3), PP2르, and the yeast kinase TOR1) that forms rod-like helical structures which function in intracellular transport (Andrade \& Bork, 1995). The C subunit is postranslationally methylated or phosphorylated. The methylation of the $\mathrm{C}$ subunit alteres binding of the regulatory $\mathrm{B}$ subunit but does not affect protein association (Yu et al., 2001). As mentioned before, Striatins appear to function as B',' family of PP2AB-type regulatory subunits. This is deducted from the lack of other regulatory PP2A subunits in the Striatin-PP2A complex and an altered substrate specify of STRIPAK-associated PP2A. To date, 17 regulatory subunits and 2 isoforms of the scaffolding 
and the catalytic subunit of PP2A are known (Cho \& Xu, 2007, Xu et al., 2006). In general, PP2A functions in various cellular processes e.g. translation and transcription, cell signaling and cell-cycle regulation (Lechward et al., 2001). This variety of functions is mediated by its regulatory B subunits, which guide the phosphatase to its target complex and modulate its activity (Moreno et al., 2000). To date, nearly all Striatin-family complexes contain PP2A subunits A and C, whereas other components are additional. Many of the STRIPAK components are phosphoproteins (Goudreault et al., 2009; Moreno et al., 2001). Moreno et al., (2001) demonstrated that among others Striatin, $\mathrm{SG}_{2} \mathrm{NA}$ and MOB3 are highly phosphorylated if PP2A is inactivated. This led to the assumption, that STRIPAK-bound PP2A is the relevant phosphatase. This hypothesis is supported by data of Gordon et al. (2011); a point mutation in the striatin gene that decreases Striatin-PP2A binding causes hyperphosphorylation of the STRIPAK-associated GCK III STK24 (Goudreault et al., 2009) (1.3.1.4). Additionally, STK25 was shown to be dephosphorylated and partially inactivated by PP2A in vitro as well as MST4 showed a gel-shift pattern similar to hyperphosphorylation after PP2A inactivation (Gordon et al., 2011, Pombo et al., 1996).

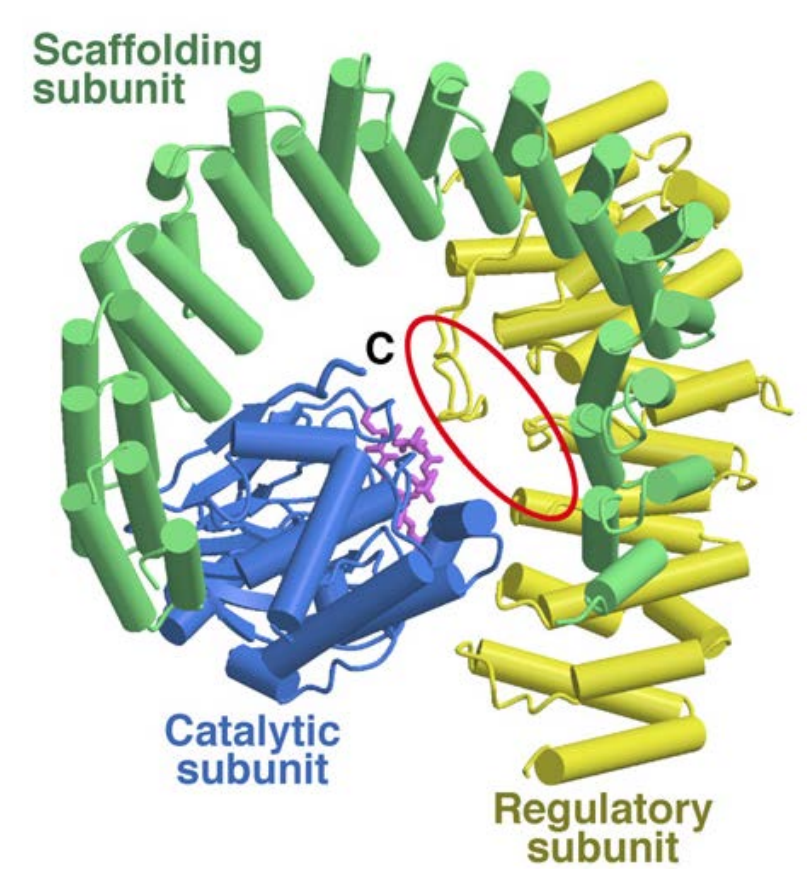

Figure 3. 3D structure of the heterotrimeric PP2A complex. Shown are the scaffolding subunit (green), the regulatory subunit (yellow) and the catalytic subunit (blue). The red circle in the center marks the absence of interactions involving the C-terminus of subunit C, shown are aa 1-294. (according to Cho and $\mathrm{Xu}$ (2007). 
Introduction

\subsubsection{MOB3}

One of the first identified interaction partners of Striatins was the 225 aa comprising MOB3 in Rattus norvegicus (Baillat et al., 2001). Similar to Striatins, MOB3 localizes primarily to somato-dendritic spines (Baillat et al., 2001, Bailly \& Castets, 2007, Trammell et al., 2008). MOB3 belongs to the group of MOB (monopolar spindle one binder) proteins. This protein group is characterized by a Mob domain, which is highly conserved and comprises 180-200 aa of the MOB protein (Baillat et al., 2001, Luca et al., 2001, Ponchon et al., 2004) (Figure 4). Crystal-structure analysis of human MOB1 protein revealed the tertiary setup of Mob domains in general (Stavridi et al., 2003). The conserved Mob domain consists of 9 helices and a hairpin, which form 2 antiparallel beta sheets. The protein forms a globular structure with a core of 4 bundled helices and the hairpin, accompanied by tetrahedrally coordinated zinc atom (Stavridi et al., 2003).

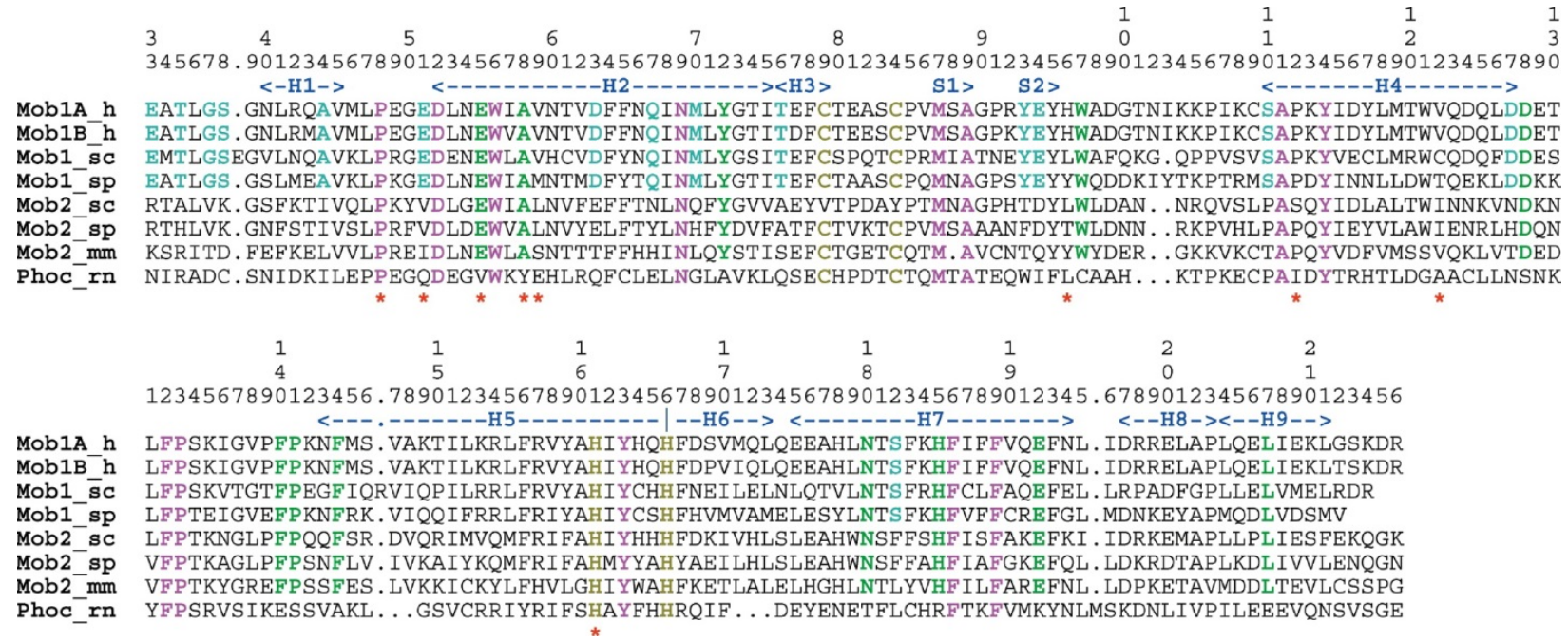

Figure 4. Sequence alignment of human MOB1 with homologs from other species. Aa-sequence alignment of human MOB1A (Mob1A_h, accession Q9H8S9) and MOB1B (Mob2B_h, Q7L9L4) aa 33216 with S. cerevisiae MOB1p (Mob1_sc, E7Q517) aa 132-314, S. pombe MOB1p (Mob1_sp, O94360) aa 31-210 and MOB2p (Mob2_sp, O74558) aa 62-243, mouse Mob2 (Mob2_mm, Q8VI63) aa 32-212 and rat phocein (Phoc_nn, Q9QYW3) aa 46-222. Human MOB1 secondary structures are displayed above the aa sequences. Residues conserved in all MOB proteins are colored in magenta, residues conserved in MOB1 and MOB2, but not MOB3 family members are colored green, MOB1-specific residues are shown in blue. The cysteine and histidine residues that surround the zinc atom are shown in gold. The asterisks below the aa sequences indicate the residues targeted by the $S$. cerevisiae MOB1 mutant alleles (modified according to Stavridi et al. (2003)). 
Introduction

One function of the Mob domain is to activate NDR (nuclear Dbf2-related) kinases. NDR kinases are essential components of cellular processes, such as cytokinesis, cell proliferation, mitotic exit and apoptosis (Chow et al., 2010). In particular, each MOB protein interacts with NDR kinases through a NDR-kinase specific, conserved N-terminal regulatory domain. This has been shown in yeast, D. melanogaster and human cell lines (Jones \& Varela-Nieto, 1998). Moreover, MOB proteins are necessary for localization of the kinases NDR1/2 in yeast and "large kinase suppressor kinase 1" (LATS 1) in D. melanogaster (Hergovich et al., 2005, Robinson, 1997). According to phylogenetic analysis of Vitulo et al. (2007) are MOB proteins divided into the 5 groups MOB1, MOB2, MOB3, MOB4 and MOBp proteins. The fruitfly $D$. menalogaster and the filamentous ascomycete $N$. crassa exhibit genes coding for $4 \mathrm{MOB}$ proteins, the human genome contains genes encoding for 7 MOB proteins (Chow et al., 2010, Maerz et al., 2009, Trammell et al., 2008). Each of them exhibits a gene that encodes a MOB3 protein, whereas a MOB3 homolog is absent in yeast. In contrast to proteins of the other MOB subgroups has MOB3 a versatile function. This is mainly based on its identified interaction partners; aside from Striatins and NDR kinases, MOB3 also interacts with epidermal growth factor receptor substrate $\underline{15}$ (EPS15) and GTPase Dynamin I involved in endocytosis and vesicular trafficking (Baillat et al., 2002). Thus, MOB3 is assumed to has a regulatory function in these processes (Hwang \& Pallas, 2014). Additionally was shown, that the MOB3 homolog in D. melanogaster is involved in spindle focusing, microtubule organization, neuronal transport and formation of synapses (Schulte et al., 2010, Trammell et al., 2008). Moreover, deletion of mob3 in the filamentous ascomycetes S. macrospora and N. crassa, led to sterility and hyphal fusion defects (Bernhards \& Pöggeler, 2011, Dettmann et al., 2013, Maerz et al., 2009). The phenotype of $\Delta$ mob3 in $N$. crassa was independent from NDR-kinases activity (Maerz et al., 2009). Thus, MOB3 has a fundamental role in developmental processes.

\subsubsection{CCM3}

CCM3 (çerebral cavernous malformation $\underline{3}$ ) also named PDCD10 (programmed cell death $\underline{10}$ ) was first identified in a premyeloid cell line and was upregulated after induced apoptosis (Bergametti et al., 2005). The name CCM3 is deducted from previous findings that mutations in this gene can cause cerebral cavernous malformations (CCM). CCMs are vascular lesions in the 
brain that are characterized by dilated vessels, abnormal in shape. These vessels lack intervening brain parenchyma and thus contain only a monolayer of endothelial cells. The symptoms caused by this malformation vary from headache to stroke (Siegel et al., 2005, Verlaan et al., 2005). The prevalence of these symptoms have been estimated to be $0.1 \%-0.5 \%$ (Otten et al., 1989). Three CCM-related genes have previously been identified, named CCM1, CCM2 and CCM3. All three genes are connected to CCM lesions (Bergametti et al., 2005). The ccm3 gene encodes 3 isoforms that only differ in their 5' region (Li et al., 2010). CCM3 is an adaptor protein of 25 $\mathrm{kDa}$ and functions among others in response to oxidative stress, vascular development, vascular endothelial growth factor signaling (VEGF) and apoptosis (Guclu et al., 2005, He et al., 2010). VEGF is a crucial factor for embryonic circulatory system development (Yla-Herttuala et al., 2007, Fidalgo et al., 2012). CCM3 is optionally acetylated at lysine residue Lys179 and exhibits a C-terminal focal adhesion targeting (FAT)-homology domain for protein-protein interaction (Choudhary et al., 2009, Li et al., 2010). CCM3 interacts with CCM2 and forms a ternary complex with CCM1 (Voss et al., 2007). Moreover, CCM3 interacts with proteins involved in cell adhesion and bound to membranes such as VEGF receptor 2 (He et al., 2010, Li et al., 2010). Furthermore, CCM3 interacts with the mammalian kinases MST4, STK24 and STK25 (Goudreault et al., 2009). MST4, STK24 and STK25 are members of the GC III kinases, a subgroup of sterile-20-like kinases (Pombo et al., 2007) (Figure 5). The interaction between the GC III kinases and CCM3 are important to prevent CCM lesions (Zheng et al., 2010). The GC III kinases function in important cellular processes such as modulation of cell death, proliferation and regulation of the cytoskeleton and Golgi morphology (Dan et al., 2002, Huang et al., 2002, Nogueira et al., 2008, Preisinger et al., 2004). CCM3 is assumed to be important for the shift of GC III kinases from the cis Golgi to the STRIPAK complex, because silencing of ccm3 reduces GCK III binding to the STRIPAK complex and increases their binding to the Golgi matrix protein GM130 (Preisinger et al., 2004). Moreover, CCM3 is essential for MST4 activation after oxidative stress; MST4 phosphorylates ERM proteins (named after their close relation to the paralogs ezrin, radixin and moesin), that crosslink actin filaments with plasma membranes, to protect cells from death. This process is impaired by inactivation of CCM3 (Lankes et al., 1988, Tsukita et al., 1997, Fidalgo et al., 2012). CCM3 was shown to be phosphorylated by STK25 and dephosphorylated by FAS-associated phosphatase FAP-1 in vitro (Voss et al., 2007). CCM3 interacts aside of Striatin and the STRIPAK-associated kinases 
MST4, STK24 and STK25 also with other proteins of this complex such as MOB3, CTTNBP2NL and STRIP1 (Goudreault et al., 2009) (Figure 2 and Figure 5). CCM3 is part of the mammalian STRIPAK core complex (Kean et al., 2011) .

A

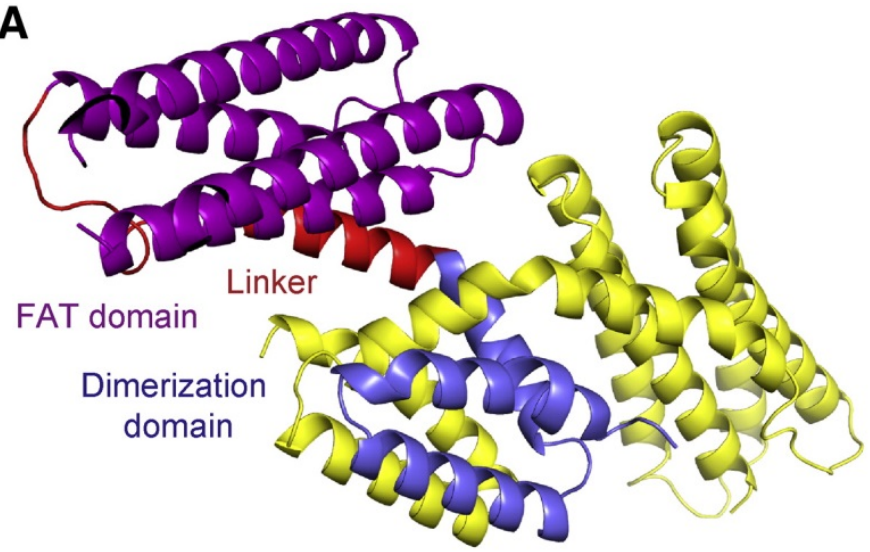

CCM3 homodimer
B

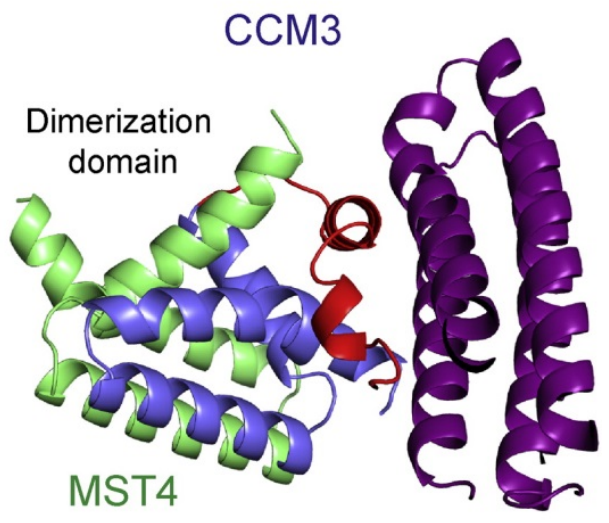

CCM3-MST4 heterodimer

C

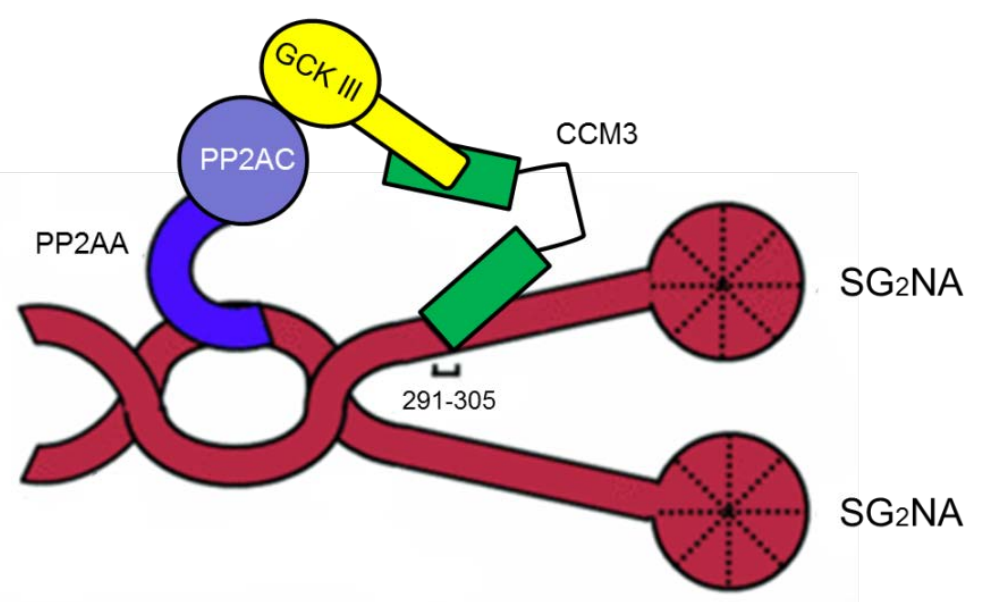

Figure 5. CCM3 functions in MST4 recruitment. (A) 3D structure of CCM3 forms a homodimer through its dimerization domain (blue). The FAT domain of CCM3 is shown in purple, the linker (aa 71-97) between these domains folds into a helix and is colored in red. The second CCM3 protein is colored in yellow. (B) CCM3 forms a heterodimer with MST4, mediated by their dimerization domains. CCM3s linker region undergoes a conformational change; its helical structure partially changes into a flexible loop in the CCM3-MST4 heterodimer. The dimerization domain of MST4 is shown in light green (according to Zhang et al. (2013)). (C) GC III kinases are bound to SG $_{2}$ NA via CCM3. PP2AA and the FAT domain of CCM3/PDCD10 interact with homo-oligomerized SG $_{2}$ NA. SG ${ }_{2}$ NA-PP2AA interaction requires the caveolin-binding domain and some of the coiled-coil domain of $\mathrm{SG}_{2} \mathrm{NA}$; CCM3 interacts with $\mathrm{SG}_{2} \mathrm{NA}$ at aa 291-305. Although $\mathrm{SG}_{2} \mathrm{NA}$ is shown, essentially the same findings apply to Striatin and presumably Zinedin (according to Kean et al. (2011)). 
Introduction

\subsubsection{The GC III kinases MST4, STK24 and STK25}

Mammalian GCKs regulate various cellular processes, such as polarization, migration, cell growth, neuronal differentiation, apoptosis and stress response (Sugden et al., 2013, Delpire, 2009). Moreover, fungal GCKs are involved in the regulation of cytokinesis, hyphal growth and differentiation of asexual structures (Boyce \& Andrianopoulos, 2011). Based on their structural similarity to the $S$. cerevisiae Sterile-20 protein kinase, GCKs are members of the Ste20-related group of protein kinases. Ste20-like kinases are subclassified into p21-ạctivated kinases (PAK) family and a GCK family depending on the location of their kinase domain (Hanks \& Hunter, 1995). The kinase domain of PAKs is located C-terminally to the regulatory domain, whereas the catalytic domain of GCKs is at the N-terminus (Dan et al., 2001). GCKs share a highly conserved catalytic domain and a poorly conserved regulatory C-terminus (Hanks \& Hunter, 1995, Dan et al., 2001). The GCK family can be further subdivided into eight families, GCK I to GCK VIII (Dan et al., 2001).

In mammals, the GC III kinases MST4, STK24, STK25 are identified as components of the STRIPAK complex (Kean et al., 2011, Gordon et al., 2011, Goudreault et al., 2009) (Figure 2). CCM3 has recently been shown to act as an adaptor to recruit GC III kinases to the Striatins (Kean et al., 2011, Gordon et al., 2011, Goudreault et al., 2009). The role of CCM3 is to bring GC III kinases in proximity to Striatin-connected phosphatase PP2A (Gordon et al., 2011) (Figure 5) It is thought that the PP2A holoenzyme including Striatin dephosphorylate GCKs and thereby reducing the catalytic activity of GCKs associated with the STRIPAK complex (Gordon et al., 2011). MST4 and STK24 have been shown to possess high basal activity (Qian et al., 2001, Schinkmann \& Blenis, 1997) but are also activated by phosphorylation. This process is facilitated by autophosphorylation and/or mitogen activated kinases cascades of a threonine residue at the activation loop (Dan et al., 2002, Huang et al., 2002, Lu et al., 2006), but only little is known about their entire regulation so far. Moreover, deletion of the $\mathrm{Ca}^{2+}$-CaM binding domain of Striatin increases the binding of MST4, STK24 and STK25, whereas binding of MOB3 was unaffected (Gordon et al., 2011). 

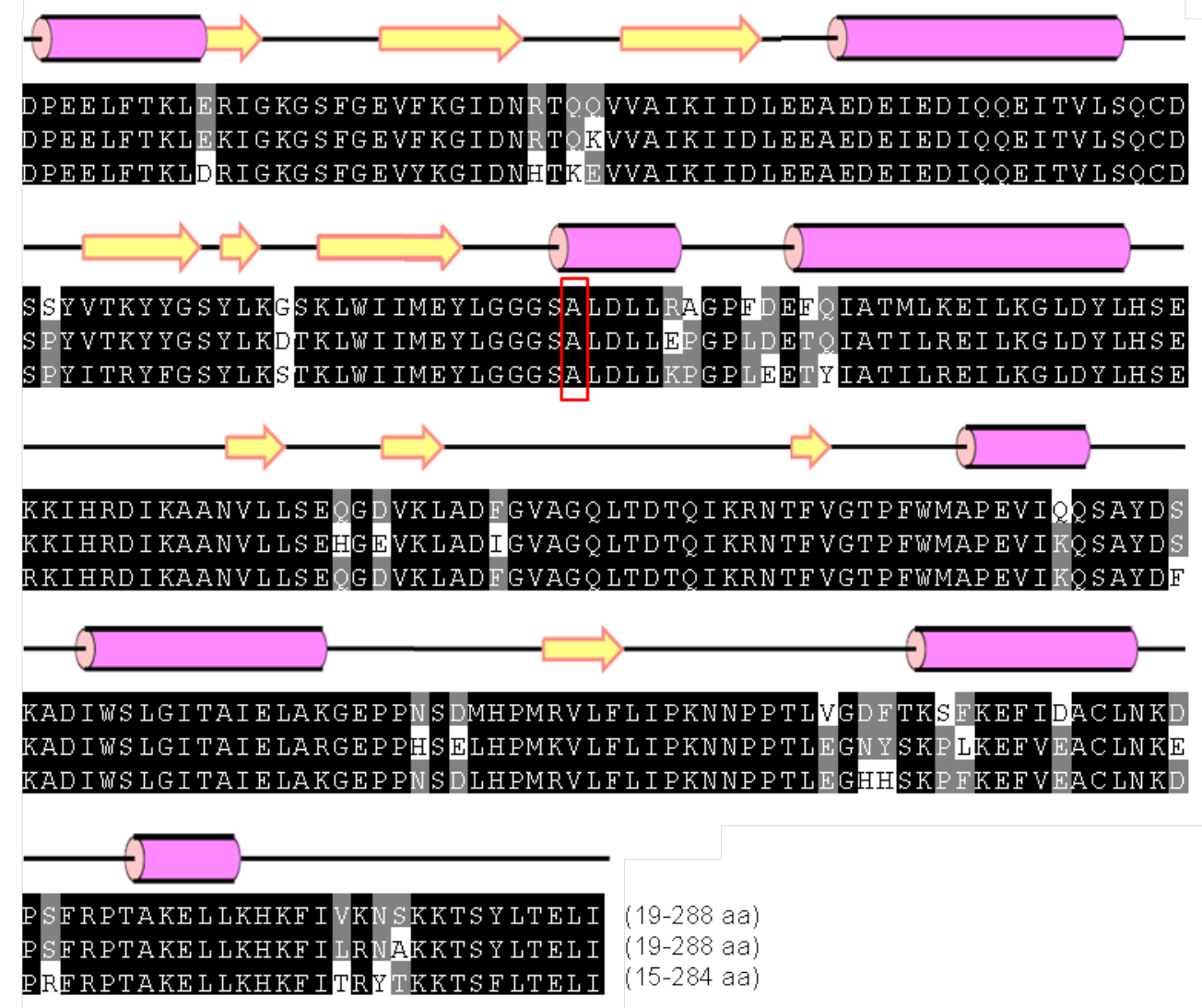

Figure 6. Aa sequence alignment, of mammalian GC III kinases. Shown are MST4 (accession number: Q9P289), MST3/STK24 (Q9Y6E0) and STK25 (O00506). The respective tertiary structure elements are shown under the aa sequence. The pink barrels mark alpha-helices; the yellow arrows represent betasheeds. The red box frames the conserved Asp residue that is involved in in ATP coordination (according to Record et al. (2010)).

\subsection{MST4}

The canonical isoform of human serine/threonine-protein kinase comprises 416 aa with a molecular weight of approximately $26.5 \mathrm{kDA}$. The mst4 gene is alternatively spliced resulting in 3 isoforms. The kinase domain of the canonical isoform is located at position 24-274, with its active side at aa 144. The ATP binding is mediated by aa 30-38. MST4 is autophosphorylated at Thr178 (Preisinger et al., 2004) (Figure 6). MST4 localizes to the Golgi apparatus and is activated by binding of Golgi matrix protein GM130, possibly as a consequence of autophosphorylation caused by stabilization of dimer formation (Preisinger et al., 2004). Moreover, MST4 is activated by the adaptor molecule MO25 (Hao et al., 2014). MO25 is conserved from yeast to man. In S. pombe, MO25 has been shown to play an essential role in 
Introduction

polarized growth and accumulation of $\mathrm{F}$-actin at the cell tip during $\mathrm{S}$ and $\mathrm{G}_{2}$ phases (Mendoza et al., 2005).

Additionally, MST4 is assumed to function in apoptotic pathways because it is cleaved by caspase-3 in vitro. MST4 functions with CCM3 in cell growth via modulating the ERK pathway (Lin et al., 2001, Ma et al., 2007) and interacts with Striatin, CCM3, CTTNBP2, SG 2 NA, CTTNBP2NL and STRIP1 (Goudreault et al., 2009).

\subsection{STK24}

Human serine/threonine-protein kinase $\underline{24}$ (STK24) is a protein of approximately $49 \mathrm{kDa}$. stk24 is alternatively spliced resulting in 2 isoforms with only slight differences. The canonical form (isoform A) comprises 443 aa in total. The kinase domain is located at position 36-286, the residues responsible for $\mathrm{Mg}^{2+}$-binding are Ala161 and Ala174.

STK24 is activated by phosphorylation either via cAMP-dependend protein kinase A (PKA) at aa 320 or autophosphorylation at aa 190. Solely, isoform B is activated by PKA (Zhou et al., 2000). These residues were verified by mutagenesis (Zhou et al., 2000, Lu et al., 2006, Olsen et al., 2006). STK24 exhibits a nuclear export signal and a bipartite nuclear localization signal (Huang et al., 2002).

Moreover, STK24 is processed by caspases; this results in kinase activation, nuclear translocation of the processed protein and induction of apoptosis (Huang et al., 2002). Similar to MST4, STK24 localizes to the Golgi apparatus where it appears to regulate cell adhesion, protein transport and neuronal migration (Matsuki et al., 2013). Furthermore, STK24 act as a major regulator of axon regeneration (Lorber et al., 2009) and interact with STRIPAK proteins Striatin, $\mathrm{SG}_{2} \mathrm{NA}$ and Zinedin, CCM3, MOB3, SLMAP, PP2A, CTTNBP2NL and STRIP1 (Ewing et al., 2007, Goudreault et al., 2009).

\subsection{STK25}

Human serine/threonine-protein kinase $\underline{25}$ is a protein of 426 aa with a molecular mass of 48 $\mathrm{kDa}$. The kinase is activated by autophosphorylation at Thr178 and ATP binding is mediated by residue 26-34 (Figure 6). Further has been shown that mutagenesis of aa 49 and 159 impairs its kinase activity (Preisinger et al., 2004). Similar to MST4, STK25 is assumed to be activated by 
Introduction

MO25 (Hao et al., 2014). STK25 is activated by oxidative stress and thus, might play a role in response to environmental stress conditions. Similar to MST4 and STK24, STK25 localizes to the Golgi apparatus where it appears to regulate cell adhesion, protein transport and neuronal migration (Matsuki et al., 2013).

\subsubsection{MINK1}

Misshapen-like kinase $\underline{1}$ (MINK1) is a serine/threonine kinase of the class of GC IV kinases. The canonical isoform of human MINK1 comprises 332 aa with a molecular weight of about 150 kDa. 5 isoforms are known, resulting from alternative splicing (Bechtel et al., 2007, Ota et al., 2004). All isoforms were found in brain tissue but with different amounts. However, isoform 1 is most abundant in skeletal muscles, isoform 2 is more abundant in brain tissue and isoform 3 and 4 are ubiquitously expressed (Hu et al., 2004). Mammalian MINK1 functions in fundamental biological processes, such as activation of mitogen-activated protein kinases in the JNK pathway, Ras-mediated p38 MAPK activation, cytoskeletal organization, cell motility and regulation of senescence (Dan et al., 2000, Hu et al., 2004, Kaneko et al., 2011, Nonaka et al., 2008). However, the explicit function of MINK1 is still unknown. Knockdown of MINK1 or Zinedin in HeLa cells resulted in multinucleated cells, caused by abnormal abscission. Moreover, PP2Amediated dephosphorylation of MINK1 is enhanced by Zinedin in vitro (Hyodo et al., 2012). Thus, Zinedin may regulate MINK1 inactivation by PP2A, similar to GC III kinases by Striatin (Gordon et al., 2011, Hwang \& Pallas, 2014). Based on gel-shift experiments were hypothesized that this process might be required for proper MINK1 function in abscission. Furthermore, the D. melanogaster homolog of MINK1 and the Striatin homolog CKA were shown to function among other biological processes in dorsal closure (Su et al., 2000, Chen et al., 2002).

\subsubsection{STRIP1/2}

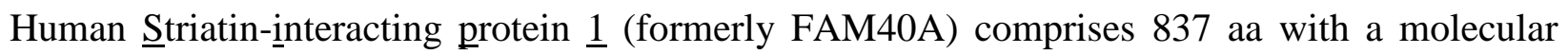
weight of approximately $96 \mathrm{kDa}$. The respective gene is alternatively spliced resulting in 4 isoforms (Bechtel et al., 2007, Ota et al., 2004). Its paralog STRIP2 (FAM40B) comprises 834 aa with a molecular weight of about $95 \mathrm{kDa}$. The strip2 gene is alternatively spliced and to date, 
Introduction

2 isoforms of STRIP2 are known (Bechtel et al., 2007, Ota et al., 2004). STRIP1/2 are membrane bound and localize to the Golgi, where they are assumed to bridge (in combination with SLMAP) the centrosome and the nuclear envelope (Frost et al., 2012). In mammals, STRIP1 and 2 were shown to interact with STRIPAK components Striatin, CTTNBP2NL and MST4 (Goudreault et al., 2009).

STRIP1/2 have a role in cytoskeletal organization, cell morphology and migration (Bai et al., 2011). Knockdown of strip1 and strip2 genes in PC3 prostate cancer cells showed different phenotypes; strip1 knockdown resulted in changed actin formation and reduced cell spreading, strip2 knockdown resulted in altered microtubule organization and induced cell elongation. Thus, STRIP1 and 2 might function in targeting STRIPAK complexes to regulate cytoskeletal function and organization (Hwang \& Pallas, 2014). Moreover, knockdown of strip1 in HeLa cells caused increased DNA content and resulted in binuclear cells. Furthermore, these nuclei were dysmorphic and fragmented. These findings indicate that STRIP1/2 function with STRIPAK components in mitotic progression and cytokinesis (Frost et al., 2012).

In $N$. crassa, the STRIP1/2 homolog HAM-2 functions in sexual development and hyphal fusion (Xiang et al., 2002). Deletion or mutation of ham-2 resulted in decreased growth velocity, shorter aerial hyphae, female sterility, and hyphal fusion defects. Furthermore was hypothesized that functional HAM-2 is needed to produce a yet unknown chemical attractant to attract conidial anastomosis tubes for hyphal fusion (Roca et al., 2005). In $N$. crassas close relative S. macrospora, STRIP1/2 homolog PRO22 was shown, aside from sexual development and hyphal fusion, to be necessary for septa formation in protoperithecia (Bloemendal et al., 2010, Rech et al., 2007).

\subsubsection{SLMAP}

The canonical isoform of human sarcolemmal membrane-âssociated protein comprises 828 aa with a molecular mass of $95 \mathrm{kDa}$. It contains a transmembrane domain, three coiled-coil domains and a forkhead-associated domain, which is a phosphopeptide recognition domain found in many regulatory proteins (Hofmann \& Bucher, 1995). To date, 8 isoforms of SLMAP are known (Wielowieyski et al., 2000, Bechtel et al., 2007, Ota et al., 2004). Isoform 1 and 2 are predominantly found in cardiac slow twitch and smooth muscles (Guzzo et al., 2004, 
Introduction

Wielowieyski et al., 2000). Each SLMAP isoforms contain one or two transmembrane domains, which are crucial for subcellular targeting (Byers et al., 2009). It is not known, which of these isoforms are STRIPAK-associated. However, based on their localization, several SLMAP isoforms have the potential to be STRIPAK-associated (Hwang \& Pallas, 2014). Guzzo et al. (2004) showed that SLMAP functions in myoblast fusion. Myoblasts are embryonic preliminary skeletal muscles. Differentiation of myoblasts induces expression of a new SLMAP isoform, whereas SLMAP isoform 1 and 3 inhibit myoblast fusion. SLMAP localization is isoformdependent (Frost et al., 2012, Guzzo et al., 2005). Moreover, a SLMAP isoform is assumed to be involved in glucose uptake (Chen \& Ding, 2011). SLMAP localizes primarily to the outer nuclear envelope, some endoplasmatic reticulum structures and to centrosomes (Frost et al., 2012, Guzzo et al., 2005, Guzzo et al., 2004). Further has been speculated, that STRIPAK components, including SLMAP have an important role in proper subcellular localization of mammalian tumor suppressor addenomatous polyposis coli (APC) (Breitman et al., 2008, Tran et al., 2013). SLMAP knockdown in HeLa cells causes slightly increased cellular DNA content and increased the amount of pericentrin foci in cells during interphase (Frost et al., 2012). Pericentrin is a component of the centrosome that serves as a multifunctional scaffold (Delaval \& Doxsey, 2010). Thus, SLMAP might function in coordination of mitotic progression and cytokinesis.

Moreover, yeast SLMAP homolog FAR9 is required for secretion of pheromone factor $\alpha$ and has been shown to function in proper sorting of proteins to the vacuole (Bonangelino et al., 2002). SLMAP has been shown to interact with Striatin, SG 2 NA, subunit A of PP2A, MST4, STK25 SIKE, STRIP1/2 and FGFR1OP2 (Goudreault et al., 2009).

\subsubsection{CTTNBP2/NL}

Human cortacțin- $\underline{b}$ inding protein $\underline{2}$ exists in two natural variants (Cheung et al., 2001). CTTNBP2 consists of 1663 aa with a molecular weight of $181 \mathrm{kDa}$. It contains a coiled-coil domain for oligomerization and 6 ankyrin repeats. These domains are common for proteinprotein interaction in nature (Mosavi et al., 2004). CTTNBP2 N-terminal-like (CTTNBP2NL) consists of 639 aa with a molecular weight of about $70 \mathrm{kDa}$ and contains a coiled-coil domain. CTTNBP2/NL are cortactin-binding proteins. Cortactin, named after cortical actin-binding protein, is a monomeric, cytoplasmatically localized protein that promotes polymerization and 
rearrangement of the actin cytoskeleton (Cosen-Binker \& Kapus, 2006). CTTNBP2 and CTTNBP2NL target cortactin to different populations of actinfibers; CTTNBP2 co-localizes with cortactin at the cell cortex, whereas CTTNBP2NL localizes to cortactin at stress fibers, with higher affinity than CTTNBP2 does (Chen et al., 2012). In contrast to CTTNBP2NL, CTTNBP2 is highly expressed in brain tissues where it regulates dendric spine density and targeting of Striatin and Zinedin to dendric spines (Chen et al., 2012). Furthermore was shown, that overexpression of cortactin but not of CTTNBP2NL or mutated CTTNBP2 impaired in cortactin binding, rescues the CTTNBP2 knockdown phenotype and thus, both proteins might have different functions (Chen et al., 2012, Chen \& Hsueh, 2012). It is further assumed that CTTNBP2 and CTTNBP2NL might guide the STRIPAK complexes to different subcellular compartments. Moreover was shown, that N-Methyl-ㅁ-aspartate (NMDA) receptor-based synaptic signaling alters the localization of Striatin and Zinedin to dendric spines but not of CTTNBP2. NMDA is an aa derivate that binds to NMDA receptors where it mimics results of glutamate binding. NMDA receptors are ion channels, that facilitate flow of small amounts of $\mathrm{Ca}^{2+}$ ions into the cell (Dingledine et al., 1999).

Treatment with NMDA resulted in decreased amounts of Striatin and Zinedin complexed with CTTNBP2, but did not affect CTTNBP2 concentrations in total (Chen et al., 2012). Activation of NMDA receptors leads to an increase of calcium concentrations in the cell. Hwang \& Pallas (2014) hypothesized on these data, that this might also cause increased CaM-Ca ${ }^{2+}$ Striatin binding. CTTNBP2 and CaM-Ca ${ }^{2+}$ share the N-terminus of Striatins for interaction and thus, $\mathrm{CaM}_{-} \mathrm{Ca}^{2+}$ might reduce CTTNBP2 binding to Striatin. CTTNBP2 and CTTNBP2NL associate with the APC-deubiquitinating enzyme Trabid (Tran et al., 2013). In addition, Striatins have been shown to be necessary for proper subcellular localization of APC (Breitman et al., 2008, Tran et al., 2013). Thus, STRIPAK might regulate APC localization and function together with Trabid (Hwang \& Pallas, 2014). CTTNBP2NL interacts with kinase MAP4K4 (Herzog et al., 2012). MAP4K4 is a negative regulator of myoblast differentiation and fusion (Wang et al., 2013). $S_{2}$ NA and Zinedin were also shown to interact with MAP4K4. Thus, CTTBNBP2NL was speculated to regulate MAP4K4 function in complex with Striatins (Hwang \& Pallas, 2014). Furthermore, CTTNBP2/NL were shown to interact with each other, Striatin, MOB3, MST4, STK24, STRIP1 and PP2A subunit A and C (Goudreault et al., 2009). 
Introduction

\subsubsection{SIKE}

The canonical isoform of human SIKE comprises 207 aa with a molecular weight of approximately $24 \mathrm{kDa}$. To date, 2 slightly different isoforms are known, both contain 2 coiledcoil domains for oligomerization. SIKE is named after suppressor of $\underline{\mathrm{IKK}} \underline{\varepsilon}$, IKKe stands for inhibitor- $\underline{\kappa} B$ kinase $\underline{\varepsilon}$ (Huang et al., 2005). IKKe and TANK-binding kinase $\underline{1}$ (TBK1) are kinases that phosphorylates interferon regulatory factor 3 (IRF-3), which is important for systemic responses to viral infections. SIKE binds to the IKK-related kinases, IKK $\varepsilon$ and TBK1, and inhibits their ability to interact with the adaptor proteins TRIF and interferon regulatory factor 3 (IRF-3), and the sensor protein retinoic acid-inducible gene $\underline{1}$ (RIG-1). TRIF is a signaling component that acts upstream of IKKe and TBK1 in toll-like receptor $\underline{3}$ (TLR3)mediated signaling (Huang et al., 2005).

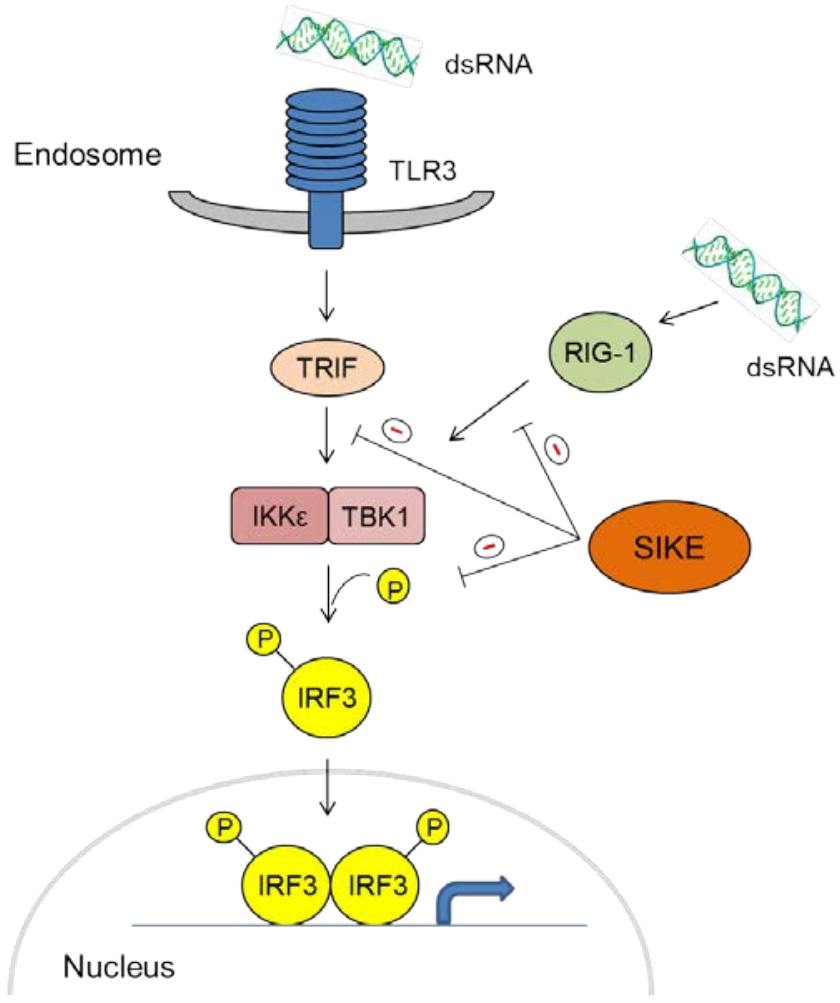

Figure 7. Function of SIKE in IKKe and TBK1-mediated innate immune response. TLR3 is activated by double-stranded RNA (dsRNA), which is generated during virus replication. This leads to interaction between TRIF and the kinases IKKe and TBK1, which phosphorylate IRF3. Phosphorylated IRF3 translocates into the nucleus, where it activates as dimer a special set of promotors. SIKE inhibits interaction between TRIF and IRF3 with IKKe and TBK1; moreover it inhibits interaction between RIG-1 and both kinases. RIG-1 functions in intracellular dsRNA recognition and activates similar to TRIF, IKK $\varepsilon$ and TBK1 (according to Cardenas (2010) and Ariumi (2014)). 
Introduction

RIG-1 functions as recognition receptor for virus detection (Pichlmair et al., 2006). Activation of TLR3 significantly reduces interaction between SIKE and TBK1 interaction (Huang et al., 2005). It is not known, if interaction of SIKE with Striatin works independently to inhibition of IKKE and TBK1. SIKE is present in various tissues, among them brain, colon, heart, lung and kidney (Huang et al., 2005).

\subsubsection{FGFR1OP2}

The canonical isoform of human fibroblast growth factor receptor $\underline{1}$ oncogene partner $\underline{2}$ (FGFR1OP2) comprises 253 aa with a molecular mass of $29.5 \mathrm{kDa}$ and contains 2 coiled-coil domains. To date, 3 isoforms are known, resulting from alternative splicing (Bechtel et al., 2007, Ota et al., 2004). FGFR1OP2 is a paralog of SIKE and was first identified in gingiva wound healing (Sukotjo et al., 2002). FGFR1OP2 is induced in wounded oral fibroblasts (Sukotjo et al., 2002). Further was shown, that FGFR1OP2 generally contributes to the ability of wound healing in fibroblasts and is important for fibroblast-cell migration (Lin et al., 2010). FGFR1OP2 is not induced in skin wounding but was shown to contribute, if endogenously expressed, to this process (Lin et al., 2010). To date, it is not known, if the STRIPAK complex functions in this process. SIKE and FGFR1OP2 are likely mutually exclusive STRIPAK members (Hwang \& Pallas, 2014).

\subsubsection{STRIPAK in signaling}

The STRIPAK complex functions in various processes among them apoptosis, cell-cycle control, signaling, Golgi assembly and vesicular trafficking, cell migration and polarity, cardiac function as well as neural and vascular development (Hwang \& Pallas, 2014). The STRIPAK core complex consists of several proteins, which function in many signaling pathway ( see 1.3.1) and interact with proteins, that in turn, functions in further pathways. Based on broad range interaction studies of the recent years, a complex model has developed (Hwang \& Pallas, 2014). Figure 8 sums up STRIPAK functions in signaling, as partially discussed under 1.3.1. Additional pathways are described in the text. 


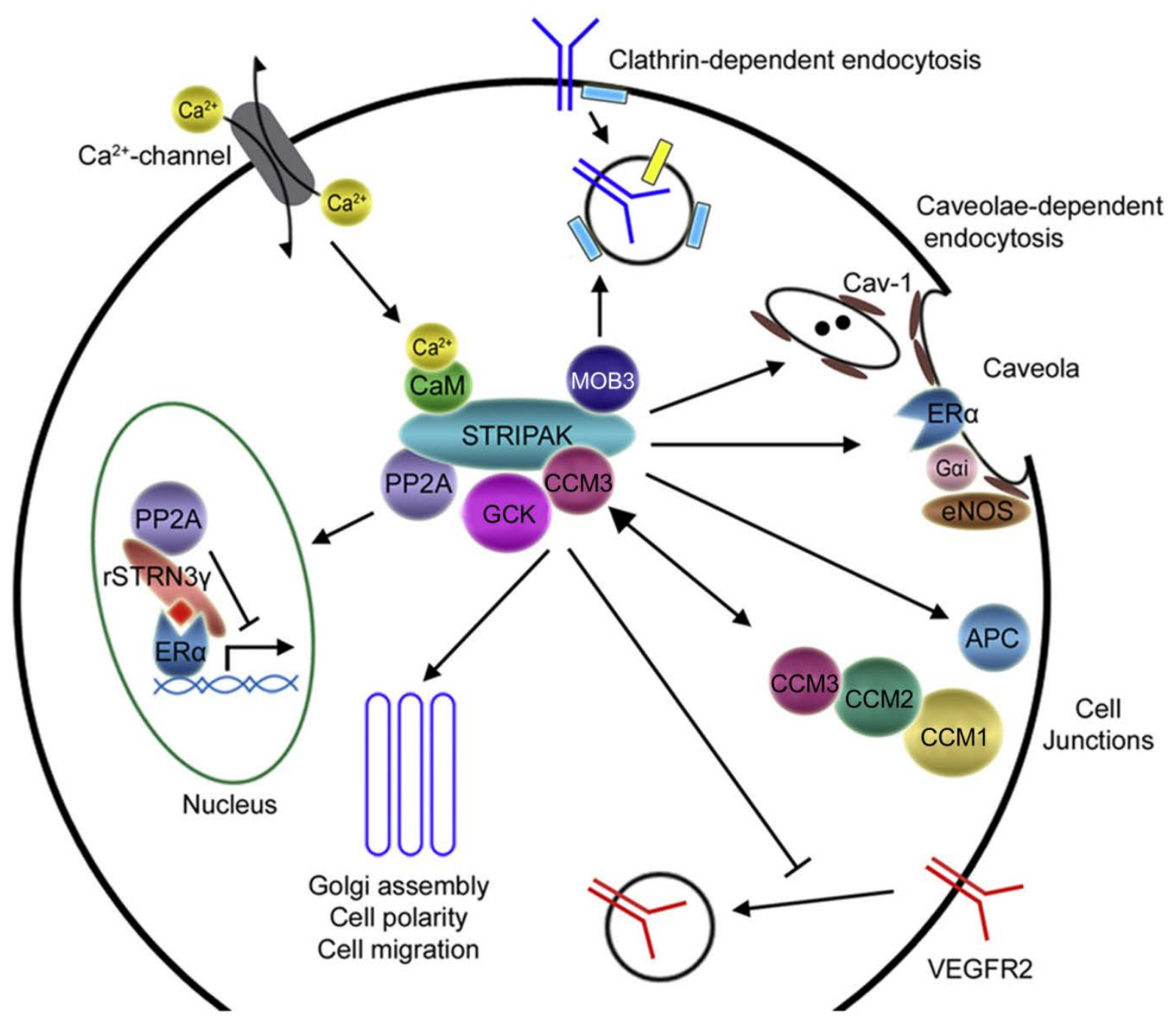

Figure 8. Schematic overview about the STRIPAK core complex in signaling. Shown is the core complex in the center and the signaling pathways it functions in indicated with arrows. The pathways are described in the following text or have been described above (according to Hwang and Pallas (2014)).

The STRIPAK-associated protein MOB3 was shown to interact with components of the clathrindependent endocytosis, such as epidermal growth factor receptor substrate $\underline{15}$ (EPS15), nucleoside-diphosphate kinase (NDPK) and Dynamin I. MOB3 was shown to co-localize with Dynamin I in neurons (Baillat et al., 2002). Generally, NDPK, EPS15, and Dynamin I function in membrane dynamics and thus, in endocytosis. EPS15 is an adaptor protein that has a role in ligand-induced receptor endocytosis of receptor-tyrosine kinases (Fazioli et al., 1993). Further was shown, that EPS15 interacts with Dynamin I physically and genetically (Baillat et al., 2002, Salcini et al., 2001). Dynamin I is a GTPase that functions in secession of clathrin-coated 
vesicles from the plasma membrane during endocytosis (Hwang \& Pallas, 2014). NDPK also physically interacts with Dynamin I (Baillat et al., 2002). Based on these findings and further research in $D$. melanogaster, NDPK is assumed to function in endocytosis by generating GTP for Dynamin I (Krishnan et al., 2001). Thus, the STRIPAK complex is hypothesized to be involved in endocytosis via MOB3 interactions. This assumption is further supported by findings in D. melanogaster, where the MOB3 homolog has a role in axonal transport, microtubule organization, neurite growth and branching and synapse assembly (Schulte et al., 2010, Sepp et al., 2008). Furthermore, the STRIPAK complex is also suggested to function in caveolaedependent endocytosis. This has already been described in section 1.1.1.

Moreover, the STRIPAK complex is implicated in regulating and targeting estrogen receptor $\underline{\alpha}$ $(\mathrm{ER} \alpha)$ to membranes. This is based identification of a STRIPAK-like complex containing ER $\alpha$, the heterotrimeric guanine nucleotide binding protein subunit Gai and endothelial nitric oxide synthetase (eNOS). Estrogens mediate cellular functions via receptor signaling (O'Lone et al., 2004). Genomic, ER $\alpha$-mediated signaling takes place at the nucleus, where ER $\alpha$ functions in transcription. Nongenomic signaling of estrogens is facilitated by caveolae-associated ER $\alpha$ and in endothelial cells including enzymes such as eNOS for rapid activation (Chambliss et al., 2000, Raz et al., 2008).

The STRIPAK complex serves as scaffolding unit for PP2A and ER $\alpha$ in nongenomic and genomic estrogen-mediated $\mathrm{ER} \alpha$ signaling (Tan et al., 2008). In rat was shown, that a $\mathrm{SG}_{2} \mathrm{NA}$ splice variant localizes to the nucleus (see also 1.1.2). This isoform forms an STRIPAK-like complex containing PP2A and ER $\alpha$ and is induced by estrogens. This complex regulates ER $\alpha$ activity by PP2A-dependent dephosphorylation (Tan et al., 2008). However, a STRIPAK complex involved in nongenomic ER $\alpha$ signaling is facilitated by direct Striatin-ER $\alpha$ interaction. Lu et al. (2004) showed that aa 1-203 of Striatin and aa 183-253 of ER $\alpha$ are sufficient for Striatin-ER $\alpha$ complex formation and aa 176-253 of Striatin are needed for estrogen-induced nongenomic eNOS activation.

Additionally, the STRIPAK complex is involved in APC-mediated organization of tight junctions via SIKE and CTTNBP2NL as described previously under 1.3.1.9 and 1.3.1.8. Tight junctions are small ribbons of membrane proteins especially found in epithelial cells, which function mainly as diffusion barrier (Bauer et al., 2014). Moreover, observation in many CCM lesions led to the hypothesis, that CCM1, CCM2 and CCM3 are important for stability or 
Introduction

assembly of cell-junctions of endothelial cells (Burkhardt et al., 2010, Clatterbuck et al., 2001, Schneider et al., 2011). Mammalian Striatin and $\mathrm{SG}_{2} \mathrm{NA}$ have been identified as interaction partners of $\underline{G A I P-i n t e r a c t i n g ~ p r o t e i n, ~} \underline{C}$ terminus (GIPC) (Varsano et al., 2006). The GTPase activating protein GAIP functions in vascular endothelial growth factor receptors $\underline{2}$ (VEGFR2) signaling, endocytosis and trafficking (Varsano et al., 2006). VEGFR2 in turn, is necessary for formation of the circulatory system (Holmes et al., 2007). Thus, the STRIPAK complex is assumed to function similar to MOB3-EPS15 interaction, in endocytosis of cell surface receptors (Hwang \& Pallas, 2014). The signaling pathways including GC III kinases and $\mathrm{Ca}^{2+}$ have already been mentioned in section 1.1 and 1.3.1.4.

The STRIPAK complex was shown to modulate signaling in the cell lumen in many ways, depending on the incoming signal, such as changed $\mathrm{Ca}^{2+}$ concentrations (Figure 8). However, incoming signals from environmental changes are first detected by receptors, which are localized at the exterior of the cell. Thus, cellular mechanisms are required that mediate signal transduction from the recognized signal at the cell exterior to the cell lumen. This process could be mediated by glycosylphosphatidylinositol (GPI)-anchored proteins, a group of proteins that are attached to the outer leaflet of the cell by a glycolipid anchor (Kinoshita, 2014). To function in this process, the GPI-anchor act as an intermediary for communication between cell exterior and internal signaling (Robinson, 1997, Jones \& Varela-Nieto, 1998). Using antibody crosslinking, some GPI-anchored proteins have been shown to effect activating or inhibitory signals, such as phosphorylation or $\mathrm{Ca}^{2+}$ influx (Robinson, 1997, Jones \& Varela-Nieto, 1998). Even though the GPI-anchored protein might not pass the cell membrane completely, it transduces incoming signals by interaction with other transmembrane proteins, such as, integrins or protein kinases (Simons \& Toomre, 2000).

\subsection{GPI-anchoring}

Eisenhaber et al. (2001) previously calculated that $0.5-1 \%$ of the total number of proteins encoded in the eukaryotic genome are GPI-anchored. Further is known that $10-20 \%$ of eukaryotic membrane proteins are attached to the cell surface by GPI-anchoring (Orlean \& Menon, 2007). These proteins are various in function such as enzymatic reactions, signal transduction, bacterial infection and cell to cell interaction (Ilangumaran et al., 2000). Further has been shown that metabolites of GPI-anchored proteins serves as second messenger in 
hormonal pathways (Young \& Moss, 2000) or mediate protein and glycoprotein endocytosis and turnover (Guo, 2013). GPI-anchors are complex glycolipids posttranslationally linked to a certain aa at the C-terminus of a group of specifically structured proteins (Eisenhaber et al., 1999) (Figure 9).

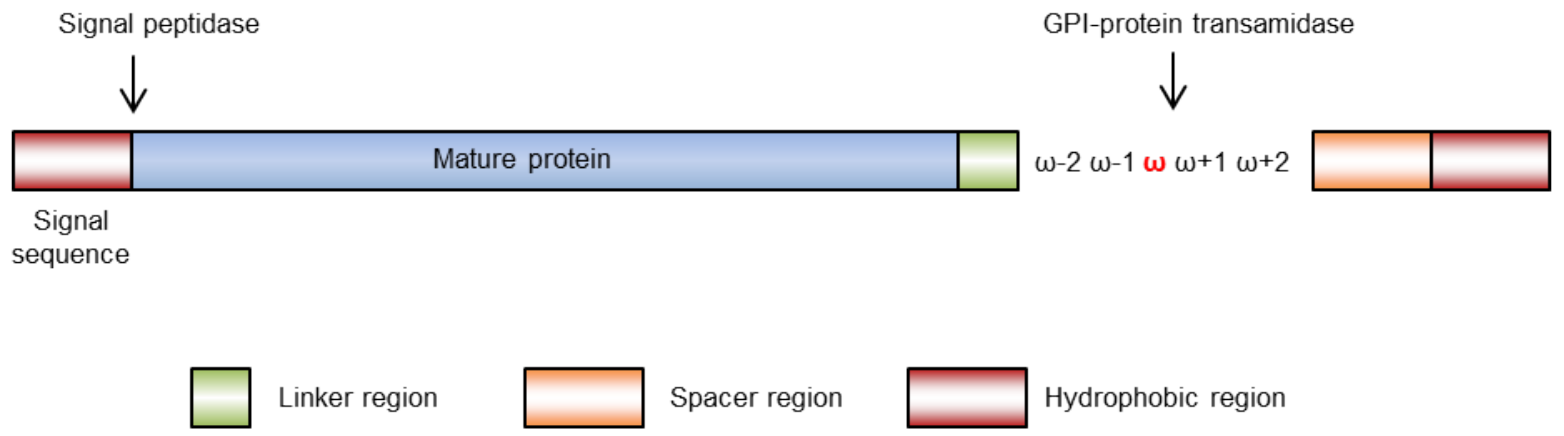

Figure 9. Schematic model of GPI-anchored protein precursors. GPI-anchored proteins are synthesized as precursors and consists of two characteristic domains, an N-terminal signal sequence and a region for GPI-anchor attachment. The signal sequence consists of hydrophobic aa and is removed by cleavage. The region for GPI-anchor attachment consists of a linker region, the $\omega$-residue, followed by a polar spacer and a hydrophobic region to the C-terminal end. The $\omega$-residue is the aa, where the GPI-anchor, a glycolipid for membrane anchoring, is attached to. GPI-anchor attachment requires transamidase cleavage of the precursor between position $\omega$ and $\omega+1$. The residues $\omega+1$ and $\omega+2$ are crucial but unknown in function. $\omega-1$ and $\omega-2$ are assumed to function in cell-wall anchoring. The Linker region separates the mature protein from the GPI-anchor (modified according to Mayor and Riezman (2004)).

The precursor of a GPI-anchored protein consists of two characteristic domains; an N-terminal signal sequence and a C-terminally located region for GPI-anchor attachment (Mayor \& Riezman, 2004) (Figure 9). The signal sequence commonly comprises 5-30 aa (Blobel \& Dobberstein, 1975) and serves for the transport to the ER (Rapoport, 2007). In the ER the protein precursor is processed (Orlean \& Menon, 2007) and the synthesis as well as the covalent attachment of the glycolipid anchor to the target proteins takes place (Pittet \& Conzelmann, 2007). The glycolipid is attached to a special residue within the sequence of the protein precursor, named $\omega$-residue. Prior to the attachment, the $\omega$-residue is exposed by transamidase cleavage (Eisenhaber et al., 1999). According to present-day research, the region for GPI-anchor attachment comprises approximately aa $\omega-10$ up to the C-terminal end of the protein precursor (Pierleoni et al., 2008). As already mentioned, the $\omega$-residue is exposed by cleavage. It has been shown that aa $\omega-1$ to $\omega+2$ are part of the transamidase cleavage side (Eisenhaber et al., 1998, 
Paladino et al., 2008). Efficient cleavage depends also on the polar spacer region and the hydrophobic tail. The spacer region encompasses the aa from position $\omega+3$ to $\omega+9$ and consists of polar residues (Pierleoni et al., 2008), the hydrophobic tail comprises aa $\omega+10$ to the $\mathrm{C}$ terminal end, represented by hydrophobic aa (Mayor \& Riezman, 2004). According to Galian et al. (2012) the hydrophobic tail was described to be "less hydrophobic than type II transmembrane anchors and more hydrophobic than the most hydrophobic segments found in secreted proteins”. After successful cleavage and glycolipid attachment to the proteins $\omega$-residue, the aa $\omega-12$ to $\omega-1$ are assumed to serve as linker between the GPI-anchor and the protein and are characterized by a low amount of secondary structure (Pierleoni et al., 2008). The region for GPI-anchor attachment might also influence the destination of the protein; aa $\omega-1$ and $\omega-2$ were previously shown to be necessary for optional cell-wall attachment in yeast (Frieman \& Cormack, 2003) and Aspergillus fumigatus (Ouyang et al., 2013). Various structures of GPIanchors have been identified (Thomas et al., 1990, Paulick \& Bertozzi, 2008, Guo, 2013) sharing the core structure of 3 mannose residues, ethanolamine, glucosamine and phosphoinositol bound to fatty acids (Paulick \& Bertozzi, 2008) (Figure 10).

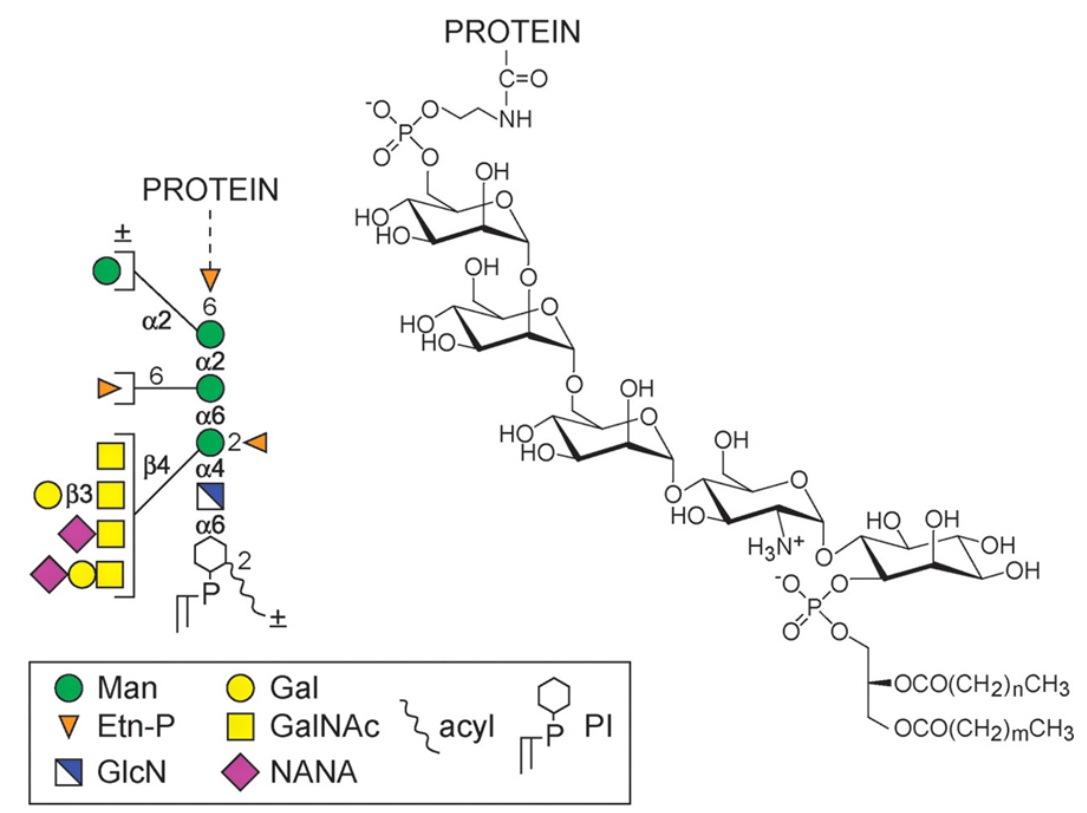

Figure 10. Structure of yeast and mammalian GPI-anchors. GPI-anchors in chemical detail are shown on the right, the schematic model is shown on the left. GPI encompasses a core of 3 mannose residues, glucosamine (GlcN), and Phosphatidylinositol (PI). The mannose residues are named Man. Man-1 is the residue linked to glucosamin $(\mathrm{GlcN})$. The third mannose residue is modified with an ethanolamine phosphate $($ Etn-P). GalNAc $=\mathrm{N}$-acetylglucosamin, NANA $=\mathrm{N}$-acetylneuraminic acid (according to Orlean \& Menon (2007)). 
Introduction

\subsection{Sordaria macrospora: A model for fruiting-body development}

The haploid filamentous ascomycete S. macropsora is an established model organism to study multicellular fruiting-body development (Teichert et al., 2014). Under natural conditions, S. macrospora grows on herbivore dung (Esser, 1992). It is self-fertile (homothallic) and lacks an asexual life cycle, meaning it solely undergoes a sexual life cycle without need for a mating partner (Pöggeler et al., 1997, Lord \& Read, 2011). Thus, mutations can be directly tested on their influence on fruiting-body formation.

Its $39.8 \mathrm{Mb}$ comprising genome is entirely sequenced, allowing easy identification of orthologs known from other organisms (Kück et al., 2009, Nowrousian et al., 2010). Further advantages are its haploidy and its largely-sized ascospores, which allow classical genetic experiments and the isolation of homokaryotic mutants after mutagenesis. The formation of multicellular fruiting bodies is an essential step in sexual reproduction of filamentous fungi and involves highly controlled cellular differentiation programs (Pöggeler et al., 2006). After the ascospores germinate, the fungus grows as two-dimensional mycelium. At day 3 of development the sexual life cycle starts with formation of ascogonia, representing female gametangia. At day 4 , these hyphal coils develop into pre-fruiting bodies, called protoperithecia. Karyogamy, meiosis, and postmeiotic mitosis occur in mature fruiting bodies (perithecia) resulting in asci with 8 linearly ordered ascospores. These spores are released from the perithecia by increased turgor pressure at day 7 as shown in Figure 11 (Engh et al., 2010, Lord \& Read, 2011).

Several PRO proteins essential for fruiting-body formation were identified in a forward genetic screen (Bloemendal et al., 2010, Dirschnabel et al., 2014, Engh et al., 2007, Masloff et al., 1999, Nowrousian et al., 2007, Nowrousian et al., 2012, Pöggeler \& Kück, 2004). Pro mutants, including pro11, are characterized by a life-cycle arrest at the stage of protoperithecia formation. The S. macrospora pro11 gene encodes a homolog to mammalian Striatin (Pöggeler \& Kück, 2004). Striatin proteins are scaffolding units of the recently identified supramolecular STRIPAK complex (Goudreault et al., 2009) (1.3). In filamentous ascomycetes, several of the yet identified homologs of mammalian STRIPAK components have been identified and are essential for fruiting-body development and cell fusion (Bloemendal et al., 2010, Maerz et al., 2009, Pöggeler \& Kück, 2004, Shim et al., 2006, Simonin et al., 2010, Xiang et al., 2002) 


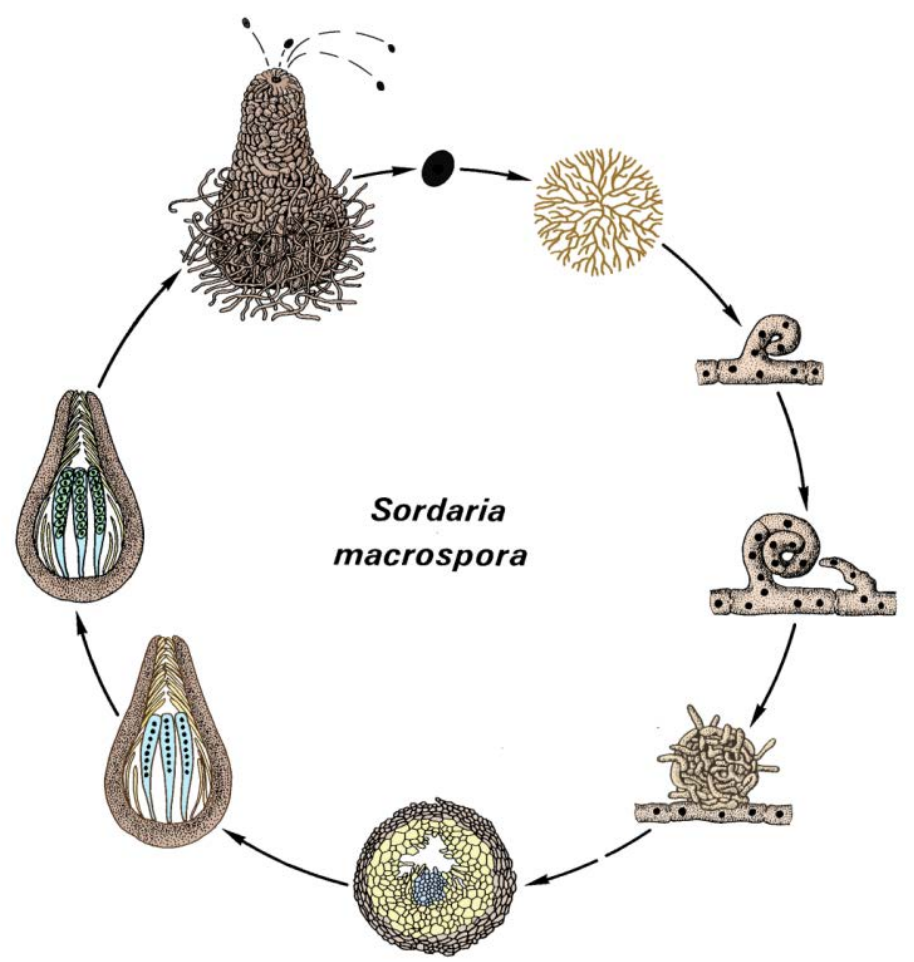

Figure 11. Schematic model of the $S$. macrospora life cycle. The life cycle starts with ascospore germination which further develops to a two-dimensional mycelium. At day 3 of development the sexual life cycle starts with formation of ascogonia. These hyphal coils develop into pre-fruiting bodies, called protoperithecia at day 4. The mature fruiting body, named perithecium contains asci with 8 linearly ordered ascospores, resulting from karyogamy, meiosis, and postmeiotic mitosis. Under laboratory conditions, the life cycle is completed after 7 day (according to Kück et al. (2009)). 
Introduction

\subsection{Aim of this study}

The central aim of this thesis was to get more insight in the role of the STRIPAK complex in the filamentous ascomycete S. macrospora. This comprised the characterization of Smgpi1, gene coding for a GPI-anchored protein which was first identified as interaction partner of SmMOB3, and the identification of potential STRIPAK-associated kinases. The potential kinases should be identified by a BLAST search against the $S$. macrospora genome using the respective mammalian kinases MST4, STK24, STK25 and MINK1 as query. Subsequently, the potential homologs should be tested for interaction with Striatin homolog PRO11. Moreover, the genes Smgpi1, and these encoding for the putative homologs of STRIPAK-associated kinases should be replaced by an $h p h$ resistance cassette to investigate their impact on sexual development and vegetative growth. The respective strains are supposed to be complemented with full-length genes and in case of $\Delta$ Smgpil, also with truncated gene versions encoding proteins comprising or lacking the signal sequence for ER targeting and the region for GPI-anchor attachment. Moreover, localization studies with fluorescence marker eGFP should be used to identify their cellular localizations. Localization of SmGPI1 should be performed with several constructs, similar to the complementation analysis but tagged with eGFP. Furthermore, generation of double-knockout strains are supposed to be generated including deletion of Smgpi1 in combination with other genes coding for STRIPAK-associated proteins PRO11 (Striatin homolog), PRO22 (STRIP1/1 homolog, SmMOB3 and PRO45 (SLMAP homolog). 


\section{Material and Methods}

\subsection{Strains}

Table 2 contains the strains used and generated for this study, ordered by organism.

Table 2. Strains generated and used for this study

\begin{tabular}{|c|c|c|}
\hline Name & Genotype & Source \\
\hline \multicolumn{3}{|c|}{ Escherichia coli } \\
\hline Mach1 & $\begin{array}{l}\Delta r e c A 139 \text { endA1 tonA } \Phi 80(\text { lacZ) } \Delta \mathrm{M} 15 \Delta l a c X 74 h s d R(r K- \\
m K+) \text {, recipient strain for vector amplification }\end{array}$ & Invitrogen, Germany \\
\hline \multicolumn{3}{|c|}{ Saccharomyces cerevisiae } \\
\hline PJ69-4A & $\begin{array}{l}\text { MATa trp1-901 leu2-3_112 ura3-52 his3_200 gal44 } \\
\text { ga18OA LYS2::GALl-HIS3 GAL2-ADE2 met2::GAL7- } \\
\text { lacZ }\end{array}$ & James et al.,1996 \\
\hline Y187 & $\begin{array}{l}\text { MATa; ura3-52; his3-200; ade2-101; trp1-901; leu2-3, } \\
\text { 112; gal4A; metA; gal80A; MEL1; URA3::GAL1 }{ }_{\text {UAS- }} \\
\text { GAL1 }{ }_{\text {TATA-lacZ }}\end{array}$ & $\begin{array}{c}\text { Clontech, Mountain View, } \\
\text { USA }\end{array}$ \\
\hline AH109 & $\begin{array}{l}\text { MATa; trp1-901; leu2-3, 112; ura3-52; his3-200; ade2- } \\
\text { 101; gal4A; gal80A; LYS2::GAL1 } 1_{U A S}-G A L 1_{\text {TATA }} \text {-HIS3; } \\
\text { GAL2 } 2_{U A S}-G A L 2_{\text {TATA }}-A D E 2 ; \text { URA3::MEL1 } 1_{U A S}-M E L 1_{\text {TATA }}- \\
\text { lacZ; MEL1 }\end{array}$ & $\begin{array}{c}\text { Clontech, Mountain View, } \\
\text { USA }\end{array}$ \\
\hline $\begin{array}{l}\text { Y187 + pBD-SmGPI1 aa } \\
1-253\end{array}$ & $\begin{array}{l}\text { MATa; ura3-52; his3-200; ade2-101; trp1-901; leu2- } \\
\text { 3,112; gal4A; met4; gal804;MEL1;URA3::GAL1 } \text { UAS }^{-} \\
\text {GAL1 }{ }_{\text {TATA-lacZ; GAL4-binding domain N-terminally }} \\
\text { fused SmGPI1 from Smgpi1 cDNA encoding aa 1-253 } \\
\text { under control of adh promoter }\end{array}$ & this study \\
\hline $\begin{array}{l}\text { Y187 + pBD-SmGPI1 aa } \\
28-227\end{array}$ & $\begin{array}{l}\text { MATa; ura3-52; his3-200; ade2-101; trp1-901; leu2- } \\
\text { 3,112; gal4A; met4; gal804;MEL1;URA3::GAL1 }{ }_{\text {UAS }}^{-} \\
\text {GAL1 }{ }_{\text {TATA-lacZ; GAL4-binding domain N-terminally }} \\
\text { fused SmGPI1 from Smgpi1 cDNA encoding aa 28-227 } \\
\text { under control of adh promoter }\end{array}$ & this study \\
\hline $\begin{array}{l}\text { Y187 + pBD-SmGPI1 aa } \\
1-100\end{array}$ & 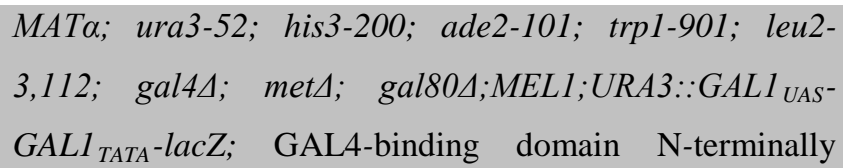 & this study \\
\hline
\end{tabular}




\begin{tabular}{|c|c|c|}
\hline & $\begin{array}{l}\text { fused SmGPI1 from Smgpi1 cDNA encoding aa 1-100 } \\
\text { under control of } a d h \text { promoter }\end{array}$ & \\
\hline $\begin{array}{l}\text { Y187 + pBD-SmGPI1 aa } \\
101-253\end{array}$ & $\begin{array}{l}\text { MATa; ura3-52; his3-200; ade2-101; trp1-901; leu2- } \\
\text { 3,112; gal4A; met4; gal804;MEL1;URA3::GAL1 }{ }_{\text {UAS }} \\
\text { GAL1 } \\
\text { fATA-lacZ; GAL4-binding domain N-terminally } \\
\text { fused SmGPI1 from Smgpi1 cDNA encoding aa 101-253 } \\
\text { under control of adh promoter }\end{array}$ & this study \\
\hline $\begin{array}{l}\text { AH109 + pAD- } \\
\text { SmMOB3 }\end{array}$ & $\begin{array}{l}\text { MATa; trp1-901; leu2-3, 112; ura3-52; his3-200; ade2 - } \\
\text { 101; gal4A; gal80A; LYS2::GAL1 } 1_{\text {UAS }}-G A L 1_{\text {TATA }} \text {-HIS3; } \\
\text { GAL2 } 2_{\text {UAS }}-G A L 2_{\text {TATA }}-A D E 2 ; \text { URA3::MEL1 } 1_{\text {UAS }}-M E L 1_{\text {TATA }} \\
\text { lacZ; MEL1; GAL4-activation domain N-terminally fused } \\
\text { to SmMOB3 from Smmob3 cDNA coding for SmMOB3 } \\
\text { full-length under control of adh promoter }\end{array}$ & this study \\
\hline $\begin{array}{l}\text { AH109 + pAD- } \\
\text { SmMOB3 aa 1-144 }\end{array}$ & $\begin{array}{l}\text { MATa; trp1-901; leu2-3, 112; ura3-52; his3-200; ade2- } \\
\text { 101; gal4A; gal80A; LYS2::GAL1 } 1_{U A S}-G A L 1_{\text {TATA }} \text {-HIS3; } \\
\text { GAL2 } 2_{U A S}-G A L 2_{\text {TATA }}-A D E 2 ; \text { URA3::MEL1 } 1_{U A S}-M E L 1_{\text {TATA }} \\
\text { lacZ; MEL1; GAL4-activation domain N-terminally fused } \\
\text { to SmMOB3 from Smmob3 cDNA coding for SmMOB3 } \\
\text { aa 1-144 under control of adh promoter }\end{array}$ & this study \\
\hline $\begin{array}{l}\text { AH109 + pAD11 aa } \\
282-845\end{array}$ & $\begin{array}{l}\text { MATa; trp1-901; leu2-3, 112; ura3-52; his3-200; ade2- } \\
\text { 101; gal4A; gal80A; LYS2::GAL1 }{ }_{\text {UAS }}-G A L 1_{\text {TATA }} \text {-HIS3; } \\
\text { GAL2 } 2_{U A S}-G A L 2_{\text {TATA }}-A D E 2 ; \quad U R A 3:: M E L 1_{U A S}-M E L 1_{\text {TATA }} \\
\text { lacZ; MEL1; GAL4-activation domain N-terminally fused } \\
\text { to PRO11 from pro11 cDNA encoding aa 282-845 under } \\
\text { control of adh promoter }\end{array}$ & this study \\
\hline AH109 + pAD11FL & $\begin{array}{l}\text { MATa; trp1-901; leu2-3, 112; ura3-52; his3-200; ade2- } \\
\text { 101; gal4A; gal804; LYS2::GAL1 } 1_{U A S}-G A L 1_{\text {TATA }} \text {-HIS3; } \\
\text { GAL2 } 2_{U A S}-G A L 2_{\text {TATA }}-A D E 2 ; \text { URA3::MEL1 } 1_{U A S}-M E L 1_{\text {TATA }} \\
\text { lacZ; MEL1; GAL4-activation domain N-terminally fused } \\
\text { to PRO11 from pro11 full-length cDNA under control of } \\
\text { adh promoter }\end{array}$ & this study \\
\hline Y187 + pBD-SmKIN3 & 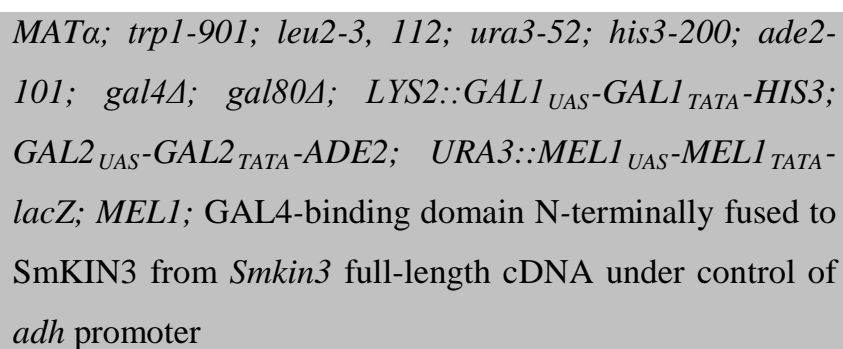 & this study \\
\hline
\end{tabular}




\begin{tabular}{|c|c|c|}
\hline Y187 + pBD-SmKIN24 & 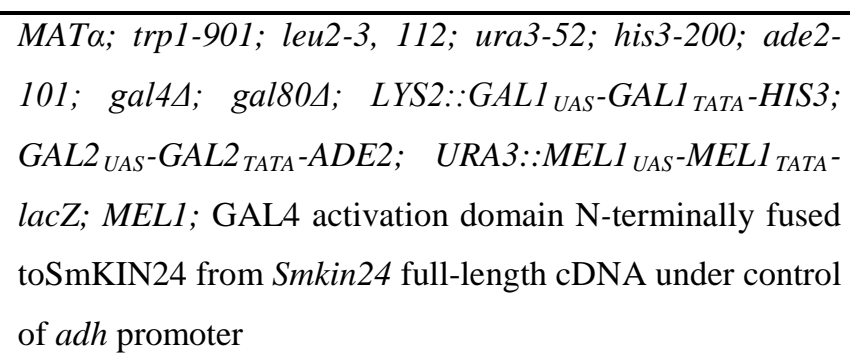 & this study \\
\hline \multicolumn{3}{|c|}{ Sordaria macrospora } \\
\hline S48977 & wild type & U. Kück, Bochuma \\
\hline S66001 & $\Delta k u 70:: n a t^{\mathrm{R}}$; fertile & Pöggeler \& Kück, 2006 \\
\hline S23442 & mutation in fus1-1 gene; brownish ascospores & Nowrousian et. al., 2012 \\
\hline S67813 & mutation in gene $r$; pink ascospores & U. Kück, Bochum ${ }^{\mathrm{a}}$ \\
\hline$\Delta$ Smkin3 & $\Delta$ Smkin3::hyg ${ }^{\mathrm{R}}$, ssi, fertile & this study \\
\hline$\Delta$ Smkin3/R2 & $\Delta$ Smkin3::hyg ${ }^{\mathrm{R}}$, pink ascospores, ssi, fertile & this study \\
\hline $\begin{array}{l}\Delta \text { SmKIN3::pRS- } \\
\text { SmKIN3+ }\end{array}$ & $\Delta$ Smkin3::hyg ${ }^{\mathrm{R}}$, pRS-SmKIN3+ ${ }^{\mathrm{ect}}$, ssi, fertile & this study \\
\hline $\begin{array}{l}\Delta \text { Smkin3::pDS- } \\
\text { SmKIN3ngfp }\end{array}$ & $\Delta$ Smkin3::hyg ${ }^{\mathrm{R}}$, pDS-SmKIN3ngfp ${ }^{\mathrm{ect}}$, ssi, fertile & this study \\
\hline$\Delta$ Smkin24 & $\Delta$ Smkin24::hyg ${ }^{\mathrm{R}}$, ssi, sterile & this study \\
\hline$\Delta$ Smkin24/R2 & $\Delta$ Smkin24::hyg ${ }^{\mathrm{R}}$, pink ascospores, ssi, sterile & this study \\
\hline $\begin{array}{l}\Delta \text { Smkin24::pRS- } \\
\text { SmKIN24+ }\end{array}$ & $\Delta$ Smkin24::hyg ${ }^{\mathrm{R}}$, pRS-SmKIN24+ ${ }^{\mathrm{ect}}$, ssi, fertile & this study \\
\hline $\begin{array}{l}\Delta \text { Smkin24::pDS- } \\
\text { SmKIN24ngfp }\end{array}$ & ASmkin24:: hyg ${ }^{\mathrm{R}}$, pDS-SmKIN24ngfp ${ }^{\text {ect }}$, ssi, fertile & this study \\
\hline$\Delta$ Smkin3/ $\Delta$ Smkin24 & $\Delta$ Smkin3::hyg ${ }^{\mathrm{R}}, \Delta$ Smkin24::hyg ${ }^{\mathrm{R}}$, ssi, sterile & this study \\
\hline S48977::pHA11 & nat $^{\mathrm{R}}$, pHA11 ${ }^{\text {ect }}$, ssi, fertile & this study \\
\hline $\begin{array}{l}\text { S67813::pFLAG- } \\
\text { SmKIN3 }\end{array}$ & pink ascospores, hyg $^{\mathrm{R}}$, pFLAG-SmKIN3 ${ }^{\text {ect }}$, ssi, fertile & this study \\
\hline $\begin{array}{l}\text { S48977::pFLAG- } \\
\text { SmKIN3, } \\
\text { S48977::pHA11 }\end{array}$ & pFLAG-SmKIN3 ${ }^{\text {ect }}$, pHA11 ${ }^{\text {ect }}$, nat ${ }^{\mathrm{R}}$, hyg ${ }^{\mathrm{R}}$, ssi, fertile & this study \\
\hline$\Delta$ Smgpil & $\Delta$ Smgpi1::hyg ${ }^{\mathrm{R}}$, ssi, fertile & Bernhards 2010 \\
\hline$\Delta$ Smgpi1/r2 & $\Delta$ Smgpi1::hyg ${ }^{\mathrm{R}}$, pink ascospores, ssi, fertile & this study \\
\hline$\Delta$ Smmob3 & $\Delta$ Smmob3::hyg ${ }^{\mathrm{R}}$, ssi, sterile & $\begin{array}{c}\text { Bernhards \& Pöggeler, } \\
2011\end{array}$ \\
\hline$\Delta \mathrm{Smmob} 3 / \mathrm{r} 2$ & $\Delta$ Smmob3:: ygg $^{\mathrm{R}}$, pink ascospores, ssi, sterile & this study \\
\hline$\Delta$ Smgpi1/ $\Delta$ Smmob3 & $\Delta$ Smgpi1::hyg ${ }^{\mathrm{R}}, \Delta$ Smmob3::hyg ${ }^{\mathrm{R}}$, ssi, fertile & this study \\
\hline
\end{tabular}




\begin{tabular}{|c|c|c|}
\hline$\Delta$ Smgpi1/ $\Delta$ Smmob3/r2 & $\begin{array}{l}\Delta \text { Smgpi1::hyg }{ }^{\mathrm{R}}, \Delta \text { Smmob3::hyg } \\
\text {, }, \text { pink ascospores, ssi, } \\
\text { fertile }\end{array}$ & this study \\
\hline$\Delta \mathrm{Sm} 3978$ & $\Delta$ Sm3978:: $h y g^{\mathrm{R}}$, ssi, fertile & this study \\
\hline$\Delta \mathrm{Smmob} 3 / \Delta \mathrm{Sm} 3978$ & $\Delta$ Smmob3:: hyg $^{\mathrm{R}}, \Delta$ Sm3978::hyg ${ }^{\mathrm{R}}$, ssi, sterile & this study \\
\hline$\Delta$ prol1 & $\Delta$ pro11::hyg ${ }^{\mathrm{R}}$, ssi, sterile & $\begin{array}{l}\text { Bernhards \& Pöggeler, } \\
2011\end{array}$ \\
\hline$\Delta$ Smgpil/ $\Delta$ pro11 & $\Delta$ Smgpi1:: yyg $^{\mathrm{R}}, \Delta$ pro11::hyg ${ }^{\mathrm{R}}$, ssi, sterile & this study \\
\hline$\Delta$ pro22 & $\Delta$ pro22::hyg ${ }^{\mathrm{R}}$, ssi, sterile & Bloemendal et al., 2012 \\
\hline$\Delta$ Smgpi1/Apro22 & $\Delta$ Smgpi1:: yyg $^{\mathrm{R}}, \Delta$ pro22::hyg ${ }^{\mathrm{R}}$, ssi, sterile & this study \\
\hline$\Delta$ pro45 & $\Delta$ pro45::hyg ${ }^{\mathrm{R}}$, ssi, sterile & U. Kück, Bochum ${ }^{\mathrm{a}}$ \\
\hline$\Delta$ Smgpi1/ $\Delta$ pro45 & $\Delta$ Smgpi1::hyg ${ }^{\mathrm{R}}, \Delta$ pro45::hyg ${ }^{\mathrm{R}}$, ssi, sterile & this study \\
\hline $\begin{array}{l}\Delta \text { Smgpi1:: } \\
\text { pRS-SmGPI1-egfp aa } 1- \\
492\end{array}$ & $\begin{array}{l}\Delta \text { Smgpi1::hyg }{ }^{\mathrm{R}}, \text { pRS-SmGPI1-egfp aa } 1-492^{\mathrm{ect}}, \text { nat }^{\mathrm{R}} \text {, ssi, } \\
\text { fertile }\end{array}$ & this study \\
\hline $\begin{array}{l}\triangle \text { Smgpi1::pRS- } \\
\text { SmGPI1-egfp aa 28-492 }\end{array}$ & $\begin{array}{l}\triangle \text { Smgpi1::hyg }{ }^{\mathrm{R}}, \mathrm{pRS}-\text { SmGPI1-egfp aa } 28-492^{\mathrm{ect}}, \text { nat }^{\mathrm{R}}, \text { ssi, } \\
\text { fertile }\end{array}$ & this study \\
\hline $\begin{array}{l}\Delta \text { Smgpil::pRS- } \\
\text { SmGPI1-egfp aa 1-466 }\end{array}$ & $\begin{array}{l}\Delta \text { Smgpi1::hyg }{ }^{\mathrm{R}}, \text { pRS-SmGPI1-egfp aa } 1-466^{\mathrm{ect}}, \text { nat }^{\mathrm{R}} \text {, ssi, } \\
\text { fertile }\end{array}$ & this study \\
\hline $\begin{array}{l}\Delta \text { Smgpi1::pRS- } \\
\text { SmGPI1-egfp aa 28-466 }\end{array}$ & $\begin{array}{l}\Delta \text { Smgpi1::hyg }{ }^{\mathrm{R}}, \mathrm{pRS}-\text { SmGPI1-egfp aa } 28-466^{\mathrm{ect}}, \text { nat }^{\mathrm{R}}, \text { ssi, } \\
\text { fertile }\end{array}$ & this study \\
\hline $\begin{array}{l}\Delta \text { Smgpi1::pRS- } \\
\text { SmGPI1-egfp aа } 1-27\end{array}$ & $\begin{array}{l}\Delta \text { Smgpi1::hyg }{ }^{\mathrm{R}}, \text { pRS-SmGPI1-egfp aa } 1-27^{\mathrm{ect}}, \text { nat }^{\mathrm{R}} \text {, ssi, } \\
\text { fertile }\end{array}$ & this study \\
\hline $\begin{array}{l}\triangle \text { Smgpil::pRS- } \\
\text { SmGPI1-egfp aa } 1-27- \\
\text { KDEL }\end{array}$ & $\begin{array}{l}\triangle \text { Smgpi1::hyg }{ }^{\mathrm{R}}, \text { pRS-SmGPI1-egfp aa 1-27-KDEL }{ }^{\mathrm{ect}} \text {, } \\
\text { nat }^{\mathrm{R}} \text {, ssi, fertile }\end{array}$ & this study \\
\hline $\begin{array}{l}\Delta \text { Smgpi1::pRS-SmGPI1 } \\
\text { aa } 1-253\end{array}$ & $\Delta$ Smgpi1::hyg ${ }^{\mathrm{R}}$, pRS-SmGPI1 aa $1-253^{\mathrm{ect}}$, nat ${ }^{\mathrm{R}}$, ssi, fertile & this study \\
\hline $\begin{array}{l}\Delta \text { Smgpi1::pRS-SmGPI1 } \\
\text { aa } 28-253\end{array}$ & $\begin{array}{l}\Delta \text { Smgpi1::hyg }{ }^{\mathrm{R}}, \mathrm{pRS}-\mathrm{SmGPI} 1 \text { aa } 28-253^{\mathrm{ect}}, \text { nat }^{\mathrm{R}} \text {, ssi, } \\
\text { fertile }\end{array}$ & this study \\
\hline $\begin{array}{l}\Delta \text { Smgpi1::pRS-SmGPI1 } \\
\text { aa } 1-227\end{array}$ & $\Delta$ Smgpi1::hyg ${ }^{\mathrm{R}}$, pRS-SmGPI1 aa $1-227^{\text {ect }}$, nat ${ }^{\mathrm{R}}$, ssi, fertile & this study \\
\hline $\begin{array}{l}\Delta \text { Smgpi1::pRS-SmGPI1 } \\
\text { aа 28-227 }\end{array}$ & $\begin{array}{l}\Delta \text { Smgpi1::hyg }{ }^{\mathrm{R}}, \text { pRS-SmGPI1 aa } 28-227^{\mathrm{ect}}, \text { nat }^{\mathrm{R}} \text {, ssi, } \\
\text { fertile }\end{array}$ & this study \\
\hline S48977::p1783 & S48977, p1783 ${ }^{\mathrm{ect}}, h_{y g^{\mathrm{R}}}$, ssi, fertile & Voigt \& Pöggeler, 2013 \\
\hline $\begin{array}{l}\text { S48977::pMOB3-FLAG } \\
\text { S48977::pRS-SmGPI1- } \\
\text { egfp aa } 1-492\end{array}$ & $\begin{array}{l}\text { S48977, pMOB3-FLAG }{ }^{\text {ect }} \text {, pRS-SmGPI1-egfp aa 1-492 }{ }^{\text {ect }} \text {, } \\
\text { hyg }^{\mathrm{R}}, \text { nat }^{\mathrm{R}} \text {, ssi, fertile }\end{array}$ & this study \\
\hline S48977::pMOB3-FLAG & S48977, pMOB3-FLAG ${ }^{\mathrm{ect}}$, hyg $^{\mathrm{R}}$, ssi & this study \\
\hline $\begin{array}{l}\text { S48977::pRS-SmGPI1- } \\
\text { egfp aa } 1-492\end{array}$ & S48977, pRS-SmGPI1-egfp aa 1-492 ${ }^{\text {ect }}$, nat ${ }^{\mathrm{R}}$, ssi, fertile & this study \\
\hline $\begin{array}{l}\text { S48977::p1783-1 } \\
\text { S48977 ::pFLAG Mob3 }\end{array}$ & S48977, p1783-1 ${ }^{\text {ect }}$, pFLAG Mob3, hyg ${ }^{\mathrm{R}}$, ssi & this study \\
\hline $\begin{array}{l}\text { S48977::pFLAGN1 } \\
\text { S48977::pRS-SmGPI1- }\end{array}$ & S48977, pFLAGN1, hyg $^{\mathrm{R}}$, pRS-SmGPI1-egfp aа $1-492^{\mathrm{ect}}$, & this study \\
\hline
\end{tabular}




\begin{tabular}{|c|c|c|}
\hline egfp aа 1-492 & $n a t^{R}$, ssi, fertile & \\
\hline $\begin{array}{l}\Delta \text { Smgpil/ } \Delta \text { Smmob3:: } \\
\text { pRS-SmGPI1 aa } 1-253\end{array}$ & $\begin{array}{l}\Delta \text { Smgpi1::hyg }{ }^{\mathrm{R}}, \Delta \text { Smmob3::hyg }{ }^{\mathrm{R}}, \text { pRS-SmGPI1 aa 1- } \\
253^{\text {ect }}, \text { nat }^{\mathrm{R}} \text {, ssi, sterile }\end{array}$ & this study \\
\hline $\begin{array}{l}\Delta \text { Smgpil/ } \triangle \text { Smmob3:: } \\
\text { pRS-SmGPI1 aa 28-253 }\end{array}$ & $\begin{array}{l}\Delta \text { Smgpil::hyg }{ }^{\mathrm{R}}, \Delta \text { Smmob3::hyg } \\
\end{array}$ & this study \\
\hline $\begin{array}{l}\Delta \text { Smgpil/ } \Delta \text { Smmob3:: } \\
\text { pRS-SmGPI1 aa } 1-227\end{array}$ & $\begin{array}{l}\Delta \text { Smgpi1::hyg }{ }^{\mathrm{R}}, \Delta \text { Smmob3::hyg }{ }^{\mathrm{R}}, \text { pRS-SmGPI1 aa 1- } \\
227^{\text {ect }} \text {, nat }{ }^{\mathrm{R}} \text {, ssi, sterile }\end{array}$ & this study \\
\hline $\begin{array}{l}\Delta \text { Smgpil/ } \triangle \text { Smmob3:: } \\
\text { pRS-SmGPI1 aa } 28-227\end{array}$ & $\begin{array}{l}\Delta \text { Smgpil::hyg }{ }^{\mathrm{R}}, \Delta \text { Smmob3::hyg }{ }^{\mathrm{R}}, \text { pRS-SmGPI1 aa 28- } \\
227^{\text {ect }} \text { nat }^{\mathrm{R}} \text {, ssi, sterile }\end{array}$ & this study \\
\hline $\begin{array}{l}\Delta \text { Smgpil/ } \Delta \text { Smmob3:: } \\
\text { pRS-SmGPI1 aa } 1-253\end{array}$ & $\begin{array}{l}\Delta \text { Smgpi1::hyg }{ }^{\mathrm{R}}, \Delta \text { Smmob3::hyg }{ }^{\mathrm{R}}, \text { pRS-SmGPI1 aa 1- } \\
253^{\text {ect }}, \text { nat }^{\mathrm{R}} \text {, ssi, sterile }\end{array}$ & this study \\
\hline $\begin{array}{l}\Delta \text { Smgpil/ } \triangle \text { Smmob3:: } \\
\text { pMobFL }\end{array}$ & $\begin{array}{l}\Delta \text { Smgpil::hyg }{ }^{\mathrm{R}}, \Delta \text { Smmob3::hyg }{ }^{\mathrm{R}}, \mathrm{pMobFL}^{\mathrm{ect}}, \text { nat }^{\mathrm{R}}, \text { ssi, } \\
\text { fertile }\end{array}$ & this study \\
\hline$\Delta$ pro11/ $\Delta$ Smkin3 & $\Delta$ pro11::hyg ${ }^{\mathrm{R}}, \Delta$ Smkin3::hyg ${ }^{\mathrm{R}}$, ssi, sterile & this study \\
\hline$\Delta$ pro11/ $\Delta$ Smkin 24 & $\Delta$ pro11::hyg ${ }^{\mathrm{R}}, \Delta$ Smkin24::hyg ${ }^{\mathrm{R}}$, ssi, sterile & this study \\
\hline
\end{tabular}

\subsection{Plasmids}

Table 3 contains the plasmids used and generated for this study with the respective features.

Table 3. Plasmids generated and used for this study

\begin{tabular}{|c|c|c|}
\hline Plasmid & Feature & Source \\
\hline pDS23-egfp & $\begin{array}{l}\text { egfp under control of gpd promoter } \\
\text { and } \operatorname{trpC} \text { terminator, ura3, nat- } \\
\text { cassette }\end{array}$ & Teichert et al., 2012 \\
\hline pRSnat & ura3, nat cassette, amp ${ }^{\mathrm{R}}$ & Klix et al., 2010 \\
\hline pRShyg & ura3, hph cassette, amp ${ }^{\mathrm{R}}$ & Bloemendal et. al 2012 \\
\hline pFLAGN1 & his-3::ccg-1(p)::3xFLAG & Kawabata \& Inoue, 2007 \\
\hline pHAN1 & his-3::ccg-1(p)::HA & Kawabata \& Inoue, 2007 \\
\hline pGBKT7 & $\operatorname{trp} 1, G A L 4-B D, k^{2} n^{\mathrm{R}}$ & Clonetech \\
\hline pGADT7 & leu2, GAL4-AD, $a m p^{\mathrm{R}}$ & Clonetech \\
\hline p1783-1 & $\begin{array}{l}\text { egfp under control of gpd } \\
\text { promoter and trpC terminator, } h p h \text { - } \\
\text { cassette }\end{array}$ & Pöggeler et. al, 2003 \\
\hline pRS-Smkin3+ & Smkin3 bp -1038 to 3811 in pRSnat & this study \\
\hline pDS-Smkin3ngfp & $\begin{array}{l}\text { Smkin3 bp } 1 \text { to } 2755 \text { in pDS23, egfp } \\
\text { is fused upstream to Smkin3 }\end{array}$ & this study \\
\hline pRS-Smkin3cgfp & $\begin{array}{l}\text { Smkin3 bp } 1 \text { to } 2755 \text { in pRSnat, egfp } \\
\text { is fused downstream to Smkin3 }\end{array}$ & this study \\
\hline pRS-Smkin24+ & Smkin24 bp -1036 to 4023 in pRSnat & this study \\
\hline pDS-Smkin24ngfp & Smkin24 bp 1 to 2947 in pDS23, & this study \\
\hline
\end{tabular}




\begin{tabular}{|c|c|c|}
\hline & egfp is fused upstream to Smkin24 & \\
\hline pRS-Smkin24cgfp & $\begin{array}{l}\text { Smkin24 bp } 1 \text { to } 2947 \text { in pRSnat, } \\
\text { egfp is fused downstream to } \\
\text { Smkin24 }\end{array}$ & this study \\
\hline pRS-KoSmkin3 & $\begin{array}{l}1038 \text { bp of the upstream region and } \\
1016 \text { bp of the downstream region of } \\
\text { Smkin3 interrupted by the } h p h- \\
\text { cassette in pRSnat }\end{array}$ & this study \\
\hline pRS-KoSmkin24 & $\begin{array}{l}1036 \text { bp of the upstream region and } \\
756 \text { bp of the downstream region of } \\
\text { Smkin24 interrupted by the } h p h- \\
\text { cassette in pRSnat }\end{array}$ & this study \\
\hline pKOSm3978 & $\begin{array}{l}988 \text { bp of the downstream region } \\
\text { and } 962 \text { bp of the upstream region of } \\
\text { Sm3978 interrupted by the } \\
\text { hph-cassette in pRSnat }\end{array}$ & this study \\
\hline pRS-SmGPI1_pre & $\begin{array}{l}\text { Smgpi1 bp -986 to } 903 \text { fused to egfp, } \\
\text { followed by Smgpi1 bp } 904 \text { to } 980 \\
\text { under control of trpC terminator in } \\
\text { pRSnat }\end{array}$ & this study \\
\hline pRS-SmGPI1-egfp aa 1-492 & $\begin{array}{l}\text { Smgpi1 bp -986 to } 869 \text { fused to egfp, } \\
\text { followed by Smgpi1 bp } 870 \text { to } 980 \\
\text { under control of trpC terminator in } \\
\text { pRSnat }\end{array}$ & this study \\
\hline pRS-SmGPI1-egfp aа 28-492 & $\begin{array}{l}\text { Smgpi1 bp }-986 \text { to }+3 \text { fused to } \\
\text { Smgpi1 bp } 82 \text { to } 901 \text { fused to egfp, } \\
\text { followed by Smgpi1 bp } 902 \text { to } 980 \\
\text { under control of trpC } C \text { terminator in } \\
\text { pRSnat }\end{array}$ & this study \\
\hline pRS-SmGPI1-egfp aa 1-466 & $\begin{array}{l}\text { Smgpi1 bp - } 986 \text { to } 901 \text { fused to egfp, } \\
\text { followed by Smgpi1 bp } 902 \text { to } 980 \\
\text { under control of trpC terminator in } \\
\text { pRSnat }\end{array}$ & this study \\
\hline pRS-SmGPI1-egfp aа 28-466 & $\begin{array}{l}\text { Smgpi1 bp -986 to } 901 \text { fused to egfp, } \\
\text { under control of } \operatorname{trp} C \text { terminator in } \\
\text { pRSnat }\end{array}$ & this study \\
\hline pDS23-SmGPI1ngfp & $\begin{array}{l}\text { Smgpi1 full-length in pDS23, egfp is } \\
\text { fused upstream to Smgpi1 }\end{array}$ & this study \\
\hline pRS-SmGPI1-egfp aa 1-27 & $\begin{array}{l}\text { Smgpi1 bp } 1 \text { to } 81 \text { fused to egfp, } \\
\text { under control of } g p d \text { promotor and } \\
\operatorname{trpC} \text { terminator in pRSnat }\end{array}$ & this study \\
\hline pRS-SmGPI1-egfp aа 1-27-KDEL & $\begin{array}{l}\text { Smgpi1 bp } 1 \text { to } 81 \text { fused to egfp, } \\
\text { followed by a short sequence } \\
\text { encoding the ER retention signal } \\
\text { KDEL under control of } g p d \\
\text { promotor and trpC terminator in } \\
\text { pRSnat }\end{array}$ & this study \\
\hline pRS-SmGPI1 aa 1-253 & Smgpi1 bp -986 to1535 in pRSnat & this study \\
\hline pRS-SmGPI1 aa 28-253 & $\begin{array}{l}\text { Smgpi } 1 \text { bp }-986 \text { to } 1 \text { fused to Smgpi1 } \\
\text { bp } 82 \text { to } 1535 \text { in pRSnat }\end{array}$ & this study \\
\hline pRS-SmGPI1 aa 1-227 & $\begin{array}{l}\text { Smgpi1 bp }-986 \text { to } 681 \text { fused to } \\
\text { Smgpi1 bp } 981 \text { to } 1535 \text { in pRSnat }\end{array}$ & this study \\
\hline pRS-SmGPI1 aa 28-227 & $\begin{array}{l}\text { Smgpi1 bp }-986 \text { to } 1 \text { fused to Smgpi1 } \\
\text { bp } 82 \text { to } 961 \text { fused to Smgpi1 bp } 981 \\
\text { to } 1535 \text { in pRSnat }\end{array}$ & this study \\
\hline pMOBFlag & 3xFLAG fused to Smmob3 under & Bloemendal et al., 2012 \\
\hline
\end{tabular}




\begin{tabular}{|c|c|c|}
\hline & control of $c c g 1$ promotor in pRShyg & \\
\hline pMOBFL & Full-length Smmob3 in pRSnat & Bernhards \& Pöggeler, 2011 \\
\hline pBD-SmGPI1 aa 1-253 & $\begin{array}{l}\text { Smgpi1 cDNA bp } 1 \text { to } 762 \text { fused to } \\
\text { GAL4-BD in pGBKT7 }\end{array}$ & this study \\
\hline pBD-SmGPI1 aa 28-227 & $\begin{array}{l}\text { Smgpi1 cDNA bp } 82 \text { to } 762 \text { fused to } \\
\text { GAL4-BD in pGBKT7 }\end{array}$ & this study \\
\hline pBD-SmGPI1 aa 1-100 & $\begin{array}{l}\text { Smgpi1 cDNA bp } 1 \text { to } 300 \text { fused to } \\
\text { GAL4-BD in pGBKT7 }\end{array}$ & this study \\
\hline pBD-SmGPI1 aа 101-253 & $\begin{array}{l}\text { Smgpi1 cDNA bp } 301 \text { to } 762 \text { fused } \\
\text { to GAL4-BD in pGBKT7 }\end{array}$ & this study \\
\hline pBD-SmKIN3 & $\begin{array}{l}\text { SmKIN3 cDNA bp 1-2463 fused to } \\
\text { GAL4-BD in pGBKT7 }\end{array}$ & this study \\
\hline pBD-SmKIN24 & $\begin{array}{l}\text { SmKIN24 cDNA bp 1-2616 fused to } \\
\text { GAL4-BD in pGBKT7 }\end{array}$ & this study \\
\hline pBD11 aa 282-845 & $\begin{array}{l}\text { pro11 cDNA bp } 843 \text { to } 2538 \text { fused } \\
\text { to GAL4 BD in pGBKT7 }\end{array}$ & this study \\
\hline pAD-SmMOB3 aа 1-144 & $\begin{array}{l}\text { Smmob3 cDNA bp } 1 \text { to } 432 \text { fused to } \\
\text { GAL4-AD in pGADT7 }\end{array}$ & this study \\
\hline pAD-SmMOB3 & $\begin{array}{l}\text { Smmob3 cDNA bp } 1 \text { to } 1992 \text { fused } \\
\text { to GAL4-AD in pGADT7 }\end{array}$ & this study \\
\hline pAD11FL & $\begin{array}{l}\text { pro11 full-length cDNA fused to } \\
\text { GAL4-AD in pGADT7 }\end{array}$ & this study \\
\hline
\end{tabular}

\subsection{Primers}

Table 4 contains the oligonucleotides used for amplification, verification or RT-PCR for this study. Primer sequences are stated in 5'-3' direction, restriction restriction recognition sequences are shown in italics.

Table 4. Primers used for this study

\begin{tabular}{|c|c|c|}
\hline Name & Sequence (5’-3’) & Binding position \\
\hline \multicolumn{3}{|c|}{ Localization, overexpression or multiple usage } \\
\hline Smkin3_5F & GACTGCCCCGGCGCGGCAGC & Smkin3 bp -929 to -910 \\
\hline Smkin3_3R & CAACGTAGGTATGTACGTAG & Smkin3 bp 3641 to 3660 \\
\hline Smkin24_F & ATGGCCGACCGCGAATATGA & Smkin24 bp 1 to 20 \\
\hline Smkin24_R & TCATGTTCCTTGTTTCATTC & $\begin{array}{l}\text { Smkin24 bp } 2928 \text { to } \\
2947\end{array}$ \\
\hline Smkin3_F & ATGGCCGACGAAGGAGTCGC & Smkin3 bp 1 to 20 \\
\hline Smkin3_R & CTAAGATCCGGCAACAGCCC & Smkin3 bp 2736 to 2755 \\
\hline 3int 1-3_R & TGCTTAATGACCTCGGGAGCCA & Smkin3 bp 822 to 841 \\
\hline 24int 1-3_R & TGCTTAATGACCTCGGGAGCCA & Smkin24 bp 540 to 563 \\
\hline 24int 4_F & CCTTCGATGCTCTATCACCAGC & $\begin{array}{l}\text { Smkin24 bp } 1801 \text { to } \\
1822\end{array}$ \\
\hline 24int 4_R & CCAGCTTATACACCAACTTGCGTATC & $\begin{array}{l}\text { Smkin24 bp } 2436 \text { to } \\
2458\end{array}$ \\
\hline Smkin3ngfp_F & $\begin{array}{l}\text { TCACTCTCGGCATGGACGAGCTGTACAAGATGGCCGAC } \\
\text { GAAGGAGTCGC }\end{array}$ & $\begin{array}{l}\text { Smkin3 bp } 1 \text { to } 20 \text {, } \\
\text { overhang to egfp }\end{array}$ \\
\hline
\end{tabular}




\begin{tabular}{|c|c|c|}
\hline Smkin3ngfp_R & $\begin{array}{l}\text { GTTTGATGATTTCAGTAACGTTAAGTGGATCATCTGTTC } \\
\text { ACCTTCTCTT }\end{array}$ & $\begin{array}{l}\text { Smkin3 bp } 2736 \text { to } 2755 \text {, } \\
\text { overhang to trpC } \\
\text { terminator }\end{array}$ \\
\hline Smkin24ngfp_F & $\begin{array}{l}\text { TCACTCTCGGCATGGACGAGCTGTACAAGATGGCCGAC } \\
\text { CGCGAATATGA }\end{array}$ & $\begin{array}{l}\text { Smkin24 bp } 1 \text { to } 20 \text {, } \\
\text { overhang to egfp }\end{array}$ \\
\hline Smkin24ngfp_R & $\begin{array}{l}\text { GTTTGATGATTTCAGTAACGTTAAGTGGATCATGTTCCT } \\
\text { TGTTTCATTC }\end{array}$ & $\begin{array}{l}\text { Smkin24 bp } 2928 \text { to } \\
\text { 2947, overhang to trpC } \\
\text { terminator }\end{array}$ \\
\hline kin3_pBD_inf_F & AGGAGGACCTGCATATGGCCGACGAAGGAGTCGC & $\begin{array}{l}\text { Smkin3 bp } 1 \text { to } 20 \text {, } \\
\text { overhang to pGBKT7 }\end{array}$ \\
\hline kin3_pBD_inf_R & GGATCCCCGGGAATTCTCATCTGTTCACCTTCTCTT & $\begin{array}{l}\text { Smkin3 bp } 2736 \text { to } 2755 \text {, } \\
\text { overhang to pGBKT7 }\end{array}$ \\
\hline kin24_pBD_inf_F & AGGAGGACCTGCATATGGCCGACCGCGAATATGA & $\begin{array}{l}\text { Smkin24 bp } 1 \text { to } 20 \text {, } \\
\text { overhang to pGADT7 }\end{array}$ \\
\hline kin24_pBD_inf_R & GGATCCCCGGGAATTCTCATGTTCCTTGTTTCATTCCC & $\begin{array}{l}\text { Smkin24 bp } 2928 \text { to } \\
\text { 2947, overhang to } \\
\text { pGADT7 }\end{array}$ \\
\hline kin3_FLAG_F & $\begin{array}{l}\text { ATTACAAGGATGACGATGACAAGGGTTCAATGGCCGAC } \\
\text { GAAGGAGTCGC }\end{array}$ & $\begin{array}{l}\text { Smkin3 bp } 1 \text { to } 20 \text {, } \\
\text { overhang to FLAG }\end{array}$ \\
\hline TtrpC_F & TCCACTTAACGTTACTGAAAT & $\begin{array}{l}\operatorname{trpC} \text { terminator bp } 1 \text { to } \\
21\end{array}$ \\
\hline pRS426GFPrev & $\begin{array}{l}\text { GCGGATAACAATTTCACACAGGAAACAGCTCGAGTGGA } \\
\text { GATGTGGAGTG }\end{array}$ & $\begin{array}{l}\text { trpC terminator bp } 749 \\
\text { to } 768 \text {, overhang to } \\
\text { pRS426 }\end{array}$ \\
\hline pRSccg1 & $\begin{array}{l}\text { GTAACGCCAGGGTTTTCCCAGTCACGACG } \\
\text { TAGAAGGAGCAGTCCA }\end{array}$ & $\begin{array}{l}\text { ccg1 promotor bp } 1 \text { to } \\
21 \text {, overhang to pRS426 }\end{array}$ \\
\hline Gpi1_5_inf_F2 & CGGGCCCCCCCTCGAGGGTCTCTGCTGCGAACCTTT & $\begin{array}{l}\text { Smgpi1 bp }-967 \text { to }-948 \text {, } \\
\text { overhang to pRS426 }\end{array}$ \\
\hline pRS4269375-4F & $\begin{array}{l}\text { GTAACGCCAGGGTTTTCCCAGTCACGACGAGGTACAAG } \\
\text { TAGTCGGCGTG }\end{array}$ & $\begin{array}{l}\text { Smgpi1 bp }-1123 \text { to } \\
1103 \text {, overhang to } \\
\text { pRS426 }\end{array}$ \\
\hline 5utr-GPI1oh-R & $\begin{array}{l}\text { GGGAATGCGACTGCTGATTATCATGACGGCAAATCTGT } \\
\text { ATTGCT }\end{array}$ & $\begin{array}{l}\text { Smgpi1 bp }-20 \text { to }-1 \text {, } \\
\text { overhang to Smgpi1 } \\
\text { ORF }\end{array}$ \\
\hline $\begin{array}{l}\text { SmGPI1core5'oh_ } \\
\text { F }\end{array}$ & $\begin{array}{l}\text { CACCTTCCAAGCAATACAGATTTGCCGTCATGCAACAA } \\
\text { CCGTTCCTGCCCG }\end{array}$ & $\begin{array}{l}\text { Smgpi1 bp } 82 \text { to } 101 \text {, } \\
\text { contains an additional } \\
\text { start codon, overhang to } \\
\text { Smgpi1 promotor }\end{array}$ \\
\hline GFP_r_TtrpCoh_R & $\begin{array}{l}\text { GTTTGATGATTTCAGTAACGTTAAGTGGATTACTTGTAC } \\
\text { AGCTCGTCCAT }\end{array}$ & $\begin{array}{l}\text { egfp bp } 694 \text { to } 713 \\
\text { (lacks a stop codon), } \\
\text { overhang to } \operatorname{trp} C \\
\text { terminator }\end{array}$ \\
\hline Omega-gfp_inf_F & GACGAGCTGTACAAGAGCGGCTTCTACTTTGGCA & $\begin{array}{l}\text { egfp bp } 1 \text { to } 20 \text {, } \\
\text { overhang to Smgpi1 } 870 \\
\text { to } 888\end{array}$ \\
\hline Core-gfp_inf_R2 & GCCCTTGCTCACCATAGGCGCACCCGTGCCAAAGT & $\begin{array}{l}\text { Smgpi1 bp } 879 \text { to } 899 \text {, } \\
\text { overhang to egfp }\end{array}$ \\
\hline Gpi1_5_R2 & GACGGCAAATCTGTATTGCT & Smgpi1 bp -20 to -1 \\
\hline GFP_F & ATGGTGAGCAAGGGCGAGGAGC & egfp bp 1 to 22 \\
\hline GFP_R & CTTGTACAGCTCGTCCATGCCGAGAGTG & egfp bp 717 to 689 \\
\hline TtrpCKDEL-F & $\begin{array}{l}\text { TGGACGAGCTGTACAAGGACGAGCTCTAAGATCCACTT } \\
\text { AACGTTAC }\end{array}$ & $\begin{array}{l}\text { trpC terminator bp } 749 \\
\text { to } 768 \text {, contains bases } \\
\text { encoding for ER } \\
\text { retention signal KDEL }\end{array}$ \\
\hline GFP9375ss-R & $\begin{array}{l}\text { GTGAACAGCTCCTCGCCCTTGCTCACCATAGCGTGGACG } \\
\text { GACAAGACCA }\end{array}$ & $\begin{array}{l}\text { Smgpi1 bp } 62 \text { to } 81 \text {, } \\
\text { overhang to egfp }\end{array}$ \\
\hline
\end{tabular}


9375_o_R

TTGATGATTTCAGTAACGTTAAGTGGATCTCAGAGAAG ACCTGACACCG
Smgpi1 bp 961 to 980 , overhang to $\operatorname{trp} C$ terminator

\section{Knockout and verification}

\begin{tabular}{ll}
\hline Smkin24_1k_5F & $\begin{array}{l}\text { GTAACGCCAGGGTTTTCCCAGTCACGACGCAGTGAGCT } \\
\text { AAGTGCTAACC }\end{array}$ \\
Smkin24_5F & GACATGCCTGCCCCACAAAT \\
Smkin24_5R & GTAACGCCAGGGTTTTCCCAGTCACGACGCGATTAAGG \\
Smkin24_3F & GAGCTGGCCTG \\
Smkin24_1k_3R & GACTTGCATAT \\
& GCGGATAACAATTTCACACAGGAAACAGCGACAGTGTA \\
& AGGGTACCTAC
\end{tabular}

$\begin{array}{ll}\text { Smkin24_2k_3R } & \text { ACTTTGATGGAAGGCTTGGTG } \\ \text { Smkin3_1k_5F } & \begin{array}{l}\text { GTAACGCCAGGGTTTTCCCAGTCACGACGCGACTCGAC } \\ \text { AGGCATGCGAA }\end{array}\end{array}$

\begin{tabular}{|c|c|c|}
\hline & & \\
\hline Smkin3_5R & $\begin{array}{l}\text { CAAAAAATGCTCCTTCAATATCAGTTAACCTTTTGGTTA } \\
\text { CAGAAGGGTG }\end{array}$ & $\begin{array}{l}\text { Smkin3 bp }-20 \text { to }-1 \text {, } \\
\text { overhang to } h p h\end{array}$ \\
\hline Smkin3_3F & $\begin{array}{l}\text { GAGTAGATGCCGACCGGGAACCAGTTAACTAGTGAGGT } \\
\text { GATGAATGGTG }\end{array}$ & $\begin{array}{l}\text { Smkin3 bp } 2756 \text { to } 2775 \text {, } \\
\text { overhang to } h p h\end{array}$ \\
\hline Smkin3_1k_3R & $\begin{array}{l}\text { GCGGATAACAATTTCACACAGGAAACAGCATCGCTTCA } \\
\text { TGACTCCCCGG }\end{array}$ & $\begin{array}{l}\text { Smkin3 bp } 3792 \text { to } 3811 \text {, } \\
\text { overhang to pRS426 }\end{array}$ \\
\hline Smkin3_2k_3R & СТTTТСССТТТTСТТСААССА & Smkin3 bp 4736 to 4755 \\
\hline Hph_F & GTTAACTGATATTGAAGGAGCATTTTTTGG & hph bp 1 to 29 \\
\hline Hph_R & GTTAACTGGTTCCCGGTCGGCATCTACTC & hph bp 1414 to 1386 \\
\hline TC1 & CACCGCCTGGACGACTAAACC & hph bp 273 to 292 \\
\hline H3 & GTACTCGCCGATAGTGGAAAC & hph bp 955-974 \\
\hline Smgpi1_3R & $\begin{array}{l}\text { GCGGATAACAATTTCACACAGGAAACAGCTTCGTTGTC } \\
\text { AGTCTAGATGG }\end{array}$ & $\begin{array}{l}\text { Smgpi1 bp } 1516 \text { to } 1535 \text {, } \\
\text { overhang to pRS426 }\end{array}$ \\
\hline Smgpi1_5F & $\begin{array}{l}\text { GTAACGCCAGGGTTTTCCCAGTCACGACGGGTCTCTGCT } \\
\text { GCGAACCTTT }\end{array}$ & $\begin{array}{l}\text { Smgpi1bp -986 to }-967 \text {, } \\
\text { overhang to pRS426 }\end{array}$ \\
\hline Pho1-14F & CCCCGACATATCGAATCCAGC & Smmob3 bp -225 to -205 \\
\hline Pho1-2R & CСССТAATGATGCСТCTACGC & Smmob3 2166 to 2146 \\
\hline $11-21$ & AAGCGCGCTTGCCAGTCGCTGC & pro11 bp -783 to -762 \\
\hline 11-Kor & ACGATCAGCCTCGGAAAGACCGC & pro11 bp 3564 to 3586 \\
\hline Pro22_ver_F & GAAGTTCGGTGGGCGATGCC & pro22 bp -801 to 820 \\
\hline Pro22_ver_R & CAAGAAGGGTCGAGATAAAG & pro22 bp 4154 to 4184 \\
\hline Pro45_ver_F & GGACCAAAGCAACGGAACGT & pro45 bp -1092 to -1111 \\
\hline Pro45_ver_R & ATACAAACCCGCTGTCGTGA & pro45 bp 3534 to 3553 \\
\hline 3978_5F & $\begin{array}{l}\text { TGGCGCGGCAAGCTGCGAGCGTAACGCCAGGGTTTTCC } \\
\text { CAGTCACGACG }\end{array}$ & $\begin{array}{l}\text { Sm3978 bp }-962 \text { to }-943 \text {, } \\
\text { overhang to pRS426 }\end{array}$ \\
\hline 3978_5R & $\begin{array}{l}\text { CAAAAAATGCTCCTTCAATATCAGTTAACATCGGAAGG } \\
\text { CGGCAGAAA }\end{array}$ & $\begin{array}{l}\text { Sm3978 bp }-20 \text { to }-1 \text {, } \\
\text { overhang to } h p h\end{array}$ \\
\hline 3978_3F & $\begin{array}{l}\text { GAGTAGATGCCGACCGGGAACCAGTTAACAGGCCTGAT } \\
\text { GCCTAGTCTTT }\end{array}$ & $\begin{array}{l}\text { Sm3978 bp } 4213 \text { to } \\
\text { 4232, overhang to } h p h\end{array}$ \\
\hline 3978_3R & $\begin{array}{l}\text { GCGGATAACAATTTCACACAGGAAACAGCCAAAGAGG } \\
\text { GAGAGGAGGTCG }\end{array}$ & $\begin{array}{l}\text { Sm3978 bp } 5201 \text { to } \\
\text { 5220, overhang to } \\
\text { pRS426 }\end{array}$ \\
\hline
\end{tabular}

Smkin24 bp -1019 to 1038 , overhang to pRS426

Smkin24 bp -1053 to $-1072$

Smkin24 bp -20 to -1, overhang to $h p h$ Smkin24 bp 2945 to 2964, overhang to $h p h$ Smkin24 bp 4004 to 4023, overhang to pRS426

Smkin24 bp 4924 to 4945

Smkin3 bp -1038 to 1019 , overhang to pRS426

Smkin3 bp -20 to -1, overhang to $h p h$ Sinkin3 bp 2756 to 2775 , overhang to pRS426 Smkin3 bp 4736 to 4755 hph bp 1 to 29 hph bp 1414 to 1386 hph bp 273 to 292 hph bp 955-974 Smgpi1 bp 1516 to 1535 , verhang to pRS426 overhang to pRS426 Smmob3 bp -225 to -205 Smmob3 2166 to 2146 pro11 bp -783 to -762 pro11 bp 3564 to 3586 pro22 bp -801 to 820 pro22 bp 4154 to 4184 pro45 bp 3534 to 3553 Sm3978 bp -962 to -943 , overhang to pRS426 m3978 bp -20 to -1, overhang to $h p h$ 4232, overhang to $h p h$ Sm3978 bp 5201 to pRS426 


\begin{tabular}{|c|c|c|}
\hline 3978_5F2 & CCAAGGCCGCAAGGCGGGCA & $\begin{array}{l}\text { Sm3978 bp }-1143 \text { to - } \\
1103\end{array}$ \\
\hline 3978_3R2 & TCGAGGGGTGCCTGGGGTCG & Sm3978 bp 5305 to 5324 \\
\hline \multicolumn{3}{|c|}{ Yeast two-hybrid } \\
\hline $\begin{array}{l}\text { Sm9375_Y2H_ges } \\
\text { _F }\end{array}$ & CATATGGATAATCAGCAGTCGCA & $\begin{array}{l}\text { Smgpi1 bp } 1 \text { to } 20, N d e I \\
\text { recognition sequence }\end{array}$ \\
\hline $\begin{array}{l}\text { Sm9375_Y2H_ges } \\
\text { _R }\end{array}$ & GААТTCTCAGAGAAGACCTGACACCG & $\begin{array}{l}\text { Smgpi1 bp } 961 \text { to } 980 \text {, } \\
\text { EcoRI recognition } \\
\text { sequence }\end{array}$ \\
\hline $\begin{array}{l}\text { Sm9375_Y2H_5’ } \\
\text { 2R }\end{array}$ & GAATTCTCAGCCGGTGCAGACGGCAT & $\begin{array}{l}\text { Smgpi1 cDNA bp } 283 \text { to } \\
\text { 303, NdeI recognition } \\
\text { sequence }\end{array}$ \\
\hline $\begin{array}{l}\text { Sm9375_Y2H_3' } \\
\text { F }\end{array}$ & САTATGAATGCCGTCTGCACCGGCAC & $\begin{array}{l}\text { Smgpi1 cDNA bp } 304 \text { to } \\
\text { 323, NdeI recognition } \\
\text { sequence }\end{array}$ \\
\hline $\begin{array}{l}\text { Sm9375_Y2H_ } \\
\text { oSigseq_F }\end{array}$ & CATATGCAACAACCGTTCCTGCCCGT & $\begin{array}{l}\text { Smgpi1 bp } 82 \text { to } 101 \text {, } \\
\text { NdeI recognition } \\
\text { sequence }\end{array}$ \\
\hline $\begin{array}{l}\text { Sm9375_Y2H_- } \\
\text { oSigseq_2R }\end{array}$ & GААТTСТCAAGGCGCACCCGTGCCAA & $\begin{array}{l}\text { Smgpi1 cDNA bp } 665 \text { to } \\
684 \text {, EcoRI recognition } \\
\text { sequence }\end{array}$ \\
\hline Mob3_Y2H_F & CATATGTCGCTTCСTCTAAGC & $\begin{array}{l}\text { Smmob3 bp } 1 \text { to } 19, \text { NdeI } \\
\text { recognition sequence }\end{array}$ \\
\hline Mob3_Y2H_R & GAАTTCCTAGTCCACCTTTGGGGCCT & $\begin{array}{l}\text { Smmob3 bp } 2078 \text { to } \\
\text { 2061, EcoRI recognition } \\
\text { sequence }\end{array}$ \\
\hline Mob3_as1-144_R & GAATTCGCA GGG CGG CTC ATC AAA CA & $\begin{array}{l}\text { Smmob3 bp } 499 \text { to } 518 \text {, } \\
\text { EcoRI recognition } \\
\text { sequence }\end{array}$ \\
\hline Pro11_Y2H_F & CATATGGGCACCAACGGCGTTCA & $\begin{array}{l}\text { pro11 bp } 1 \text { to } 20, \text { NdeI } \\
\text { recognition sequence }\end{array}$ \\
\hline Pro11_Y2H_R & GAАTTCTCACCTCGCATACACCTTGACC & $\begin{array}{l}\text { pro11 bp } 2696 \text { to } 2716 \text {, } \\
\text { recognition sequence }\end{array}$ \\
\hline $\begin{array}{l}\text { Pro11_WD40_Y2H } \\
\text { _R }\end{array}$ & CATATGGGACAACAGCTACACGATATTC & $\begin{array}{l}\text { pro11 bp } 968 \text { to } 986 \text {, } \\
\text { NdeI recognition } \\
\text { sequence }\end{array}$ \\
\hline
\end{tabular}

\section{Southern hybridization}

\begin{tabular}{|c|c|c|}
\hline Pho1-3F & AGCACAGCGAACACAAGAGG & $\begin{array}{l}\text { Smmob3 bp } 2748 \text { to } \\
2762\end{array}$ \\
\hline Pho1-3R & AGCCTAGTCCACCTTTGGGGCCT & $\begin{array}{l}\text { Smmob3 bp } 2748 \text { to } \\
2762\end{array}$ \\
\hline 9375_Sprobe_F & CCGGTCTCGCGGGCACCAAC & Smgpi1 bp -432 to -413 \\
\hline Kin3_probe_F & GGTGATGAATGGTGAAGAGA & Smkin3 bp 2761 to 2780 \\
\hline Kin3_probe_R & GTGATGAGAAAAGCGTGAATAG & Smkin3 bp 448 to 467 \\
\hline Kin24_probe_F & GTTAAAGGGGGACACCGGGG & $\begin{array}{l}\text { Smkin24 bp } 2976 \text { to } \\
2996\end{array}$ \\
\hline Kin24_probe_R & GACAAGAGGACAGTAGGTACACG & Smkin24 bp 559 to 536 \\
\hline
\end{tabular}




\subsection{Chemicals and Materials}

Acetic acid (Roth GmbH, 3738.2), acrylamide (Rotiphorese ${ }^{\circledR}$ Gel 40 37,5:1) (Roth GmbH, 3029.1), adenine (Sigma-Aldrich, 01830-50G), AEBSF (4-(2-aminoethyl)benzensulfonylfluorid) (AppliChem, A1421,0001), agar-agar (Roth GmbH, 5210.2), agar-agar SERVA high gel-strength (SERVA, 11396.03), agarose (Biozym Scientific GmbH, 840004), ammonium chloride (VWR International, BDH0208-500G), ammonium sulfate (AppliChem, A1032,1000), ampicillin (Sigma-Aldrich, A9518-25G), arginine (AppliChem, A3709,0250), ammonium persulfate (APS) (Roth GmbH, 9592.3), aprotinin (AppliChem, A232,0025), bacto-yeast-extract (Oxoid LTD., LP0021), ß-glycerophosphat (AppliChem, A2253,0100), benzamidine (AppliChem, A1380,0005), bio malt maize extract (Brau-Partner Kling, 115), biotin (SigmaAldrich, B4501-1G), bismaleimidohexane (Thermo Scientific, 22330), boric acid (Roth GmbH, 6943.1), bromophenol blue (AppliChem, A3640,0005), caffeine anhydrous (Roth GmbH, N815.3), calcium chloride (Roth GmBH, CN92.1), calcium chloride dihydrate (Roth GmbH, 5239.1), chloroform (Merck Millipore, 1024451000), citric acid monohydrate (Roth GmbH, 3958.1), copper (II) sulfate 5-hydrate (Roth GmbH, P024.1), Corning® Spin-X ${ }^{\circledR}$ UF concentrators (Corning, 431489), coomassie brilliant blue G-250 (Roth GmbH, 9598.1), coomassie brilliant blue R-250 (Roth GmbH, 3862.1) CSM-Ade-His-Leu-Trp-Ura (MP Biomedicals, 4550-122), DAPI (4'-6-diamidino-2-phenylindole) (AppliChem, A1001,0010), deoxycholic acid sodium (Roth GmbH, D6750), desoxynucleotid triphosphate (dNTPs) (Thermo Scientific, R0191), Difco ${ }^{\mathrm{TM}}$ skim milk (BD Biosciences, 232100), Difco ${ }^{\mathrm{TM}}$ Yeast Nitrogen Base w/o amino acids and ammonium sulfate (BD Biosciences, 233520), dimethylformamide (Roth GmbH, T921.1), di-sodium hydrogen phosphate (Merck-Millipore, 1065855000), DMSO (dimethyl sulfoxide) (Merck Millipore, 1029310500), DTT (1,4-dithiothreitol) (AppliChem, A1101,0025), EDTA (ethylenediamine tetraaceticacid disodium salt dihydrate) (Roth GmbH, 8043.2), electroporation cuvettes (VWR International, 732-1137), ethanol (VWR International, 20821.321), ethidium bromide (Sigma-Aldrich, 46065), Flat Optical 8-Cap Strip $0.2 \mathrm{ml}$ (Biozym, 712100), FM 4-64 (Invitrogen, F34653), formaldehyde (Roth GmbH, 4979.2), formamide (Sigma-Aldrich, 47670), formic acid (Merck Millipore, 1002641000), Gene Ruler DNA Ladder Mix (Thermo Scientific, SM0331, GeneScreen Hybridization Transfer Membrane (PerkinElmer Lifesciences, NEF988001PK), GfpTrap_A (Chromotek, gta-20), glass beads $\varnothing$ 0.25-0.5 mm (Roth GmbH, A553.1), glass beads Ø 2.85-3.45 mm (Roth GmbH, A557.1), 
glucose (AppliChem, A3617,1000), glycine (Roth GmbH, 0079.1), glycerine (VWR International, 24388.295), HEPES (4-(2-hydroxyethyl)-1-piperazineethanesulfonicacid) (Roth GmbH, 9105.4), histidine (Merck Millipore, 1.04351.0025), hydrochloric acid (Roth GmbH, 4625.2), hydrogen peroxide 30\% $\left(\mathrm{H}_{2} \mathrm{O}_{2}\right)$ (Merck Millipore, 8.22287.2500), hygromycin B (Merck-Millipore, 400051-10MU), IPTG (isopropyl- $\beta$-D-galactopyranoside) (Roth GmbH, 2316.3), iron (II) chloride (Roth $\mathrm{GmbH}, 231-753-5$ ), iron(II) sulfate heptahydrate (SigmaAldrich, 31236), imidazole (Roth GmbH, X998.1), isopropanol (AppliChem, A0900,2500GL), kanamycin sulfate (Sigma-Aldrich, 60615), leucine (AppliChem, A1426,0100), leupeptin (Nacetyl-L-leucyl-L-leucyl-L-argininal) (AppliChem, A2183,0025), lithium acetate (Roth GmbH, 5447.1), maize flour (Mühle Levers, Bochum, Germany), magnesium chloride hexahydrate (Merck Millipore, 1.05833.1000), magnesium sulfate heptahydrate (Roth GmbH, P027.2), manganese (II) chloride tetrahydrate (Roth GmbH, T881.1), manganese (II) sulfate monohydrate (Roth GmbH, 4487.1), menadione sodium bisulfite (Sigma-Aldrich, M5750), methanol (VWR International, 20864.320), n-dodecyl-ß-D-maltoside (AppliChem, A0819,0500), MitoTracker ${ }^{\circledR}$ Red FM (life technologies) M22425, MOPS (3-(N-Morpholino)-propane sulfonic acid) (AppliChem, A2947,0500), Nitrocellulose Transfer Membrane Protran ${ }^{\circledR}$ BA (Whatman, 10401196), Nonident ${ }^{\circledR}$ P40 (AppliChem A2239,0025), nourseothricin (WernerBioAgents, 5004000), PEG 6000 (Sigma-Aldrich, 81255), pepstatin A (AppliChem, 2205,0025), phenol (AppliChem, A1153,0500), phosphoric acid (Roth GmbH, 6366.1), PMFS (phenylmethylsulfonylfluoride) (Sigma-Aldrich, P-7626), potassium acetate (Merck Millipore, 1.04820.1000), potassium chloride (AppliChem, A3582,1000), potassium dihydrogen phosphate (Merck Millipore, 1.04873.1000), potassium hydroxide (Roth GmbH, 6751.1), potassium nitrate (Merck Millipore, 1.05063.1000), potassium nitrite (Sigma-Aldrich, 31443), RNA Loading Dye (2x) (Thermo Scientific, R0641), Rotiphorese Gel 40 (Roth GmbH, 3030.2), SDS (sodium dodecyl sulfate) (Roth GmbH, 4360.2), sepharose A (GE Healthcare, 17-0780-01), sodium acetate (Roth GmbH, 6773.2), sodium chloride (AppliChem, A3597,1000), sodium dihydrogen phosphate monohydrate (Merck Millipore, 1.06346.1000), sodium fluoride (AppliChem, A0401,0100), sodium hydroxide (VWR International, 28244.295), sodium molybdate-dihydrate (Sigma-Aldrich, 31439), sodium orthovanadate (AppliChem, 2196,0005), sorbitol (Roth GmbH, 6213.1), $\beta$-mercaptoethanol (Roth GmbH, 4227.1), sterile filter 0.45/0.2 $\mu \mathrm{m}$ (Sarstedt, 83.1826/83.1826.001), sucrose (AppliChem, A4734,1000), TEMED (N,N,N',N'- 
tetramethylethylenediamine) (Roth GmbH, 2367.3), theophylline (Sigma-Aldrich, T1633), thiourea (Roth GmbH, HN37.1), Tris (Tris-hydroxymethyl-aminomethane) (Roth GmbH, AE15.2), Tris/HCl (Roth GmbH, 9090.3), Triton X-114 (Sigma-Aldrich, X114), Trizol (Invitrogen,15596026), tryptone/peptone (Roth GmbH, 8952,2), tryptophan (MP Biomedicals, 4061-012), Tween 20® (AppliChem, A4974,0100 ), uracil (MP Biomedicals, 4061-212), urea (Roth GmbH, 2317.3), Whatman Paper B002 580x600 mm (Schleicher \& Schuell, 88-3852), XGal (5-bromo-4-chloro-3-indolyl-beta-D-galacto-pyranoside) (Thermo Scientific, R0404), X-ray films (Fujifilm, 4741019236), xylene Cyanol (AppliChem, A4976,0005), yeast extract (Roth GmbH, 2904.1), zinc chloride (Sigma-Aldrich, 14424), zinc sulfate heptahydrate (Roth GmbH, K301.1)

\subsection{Enzymes}

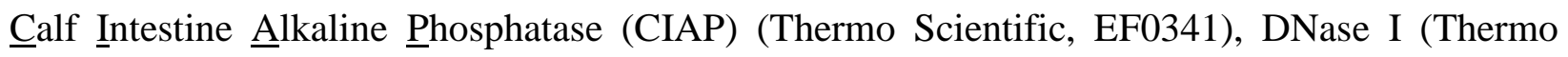
Scientific, EN0521), natuzym (Schliessmann, 5090), HotstarTaq MasterMix (Qiagen, 203443), lysozyme (SERVA, 28262.03), Moltaq (Molzym GmbH and Co, P-010-1000), Phospholipase C (Invitrogen, P6466), Pfu polymerase (Promega GmbH, M7741), Phusion ${ }^{\circledR}$ Hot Start High-

Fidelity DNAPolymerase (New England Biolabs, M0530S), restriction endonucleases (Thermo Scientific), RNase A (AppliChem, A2760,0100), T4 DNA ligase (Thermo Scientific, EL0011)

\subsection{Kits}

CloneJET PCR Cloning Kit (Thermo Scientific, K1231), High Prime DNA Labelling and Detection Starter Kit II (Roche, 1585614), HiSpeed Plasmid Midi Kit (Qiagen, 12643), InFusion ${ }^{\circledR}$ HD Cloning Kit (Clontech, 639648), Protein Deglycosylation Mix (NEB, P6039S), Qiagen PCR Cloning Kit (Qiagen, 231124), QIAprep Spin Miniprep Kit (Qiagen, 27106), QIAquick Gel Extraction Kit (Qiagen, 28704), QIAquick PCR Purification Kit (Qiagen, 28104), Substrat HRP Immobilon Western (Merck Millipore WBKLS0500), Transcriptor High Fidelity cDNA Synthesis Kit (Roche, 05091284001) 


\subsection{Media and Solutions}

\subsubsection{Solutions}

\subsubsection{Amino-acid stock solutions}

Adenine: $\quad 0.02 \%(\mathrm{w} / \mathrm{v})$ adenine in $\mathrm{H}_{2} \mathrm{O}$

Histidine: $\quad 1 \%(\mathrm{w} / \mathrm{v})$ histidine in $\mathrm{H}_{2} \mathrm{O}$

Leucine: $\quad 1 \%(\mathrm{w} / \mathrm{v})$ leucine in $\mathrm{H}_{2} \mathrm{O}$

Tryptophan: $\quad 0.02 \%(\mathrm{w} / \mathrm{v})$ tryptophan in $\mathrm{H}_{2} \mathrm{O}$

Uracil: $\quad 0.02 \%(w / v)$ uracil in $\mathrm{H}_{2} \mathrm{O}$

Trace-element stock solution:

$5 \%$ (w/v) citric acid $\left(\mathrm{C}_{6} \mathrm{H}_{8} \mathrm{O}_{7}\right.$ monohydrate), 5\% (w/v) $\mathrm{ZnSO}_{4}$ heptahydrate, 1\% (w/v) $\mathrm{Fe}\left(\mathrm{NH}_{4}\right)_{2}\left(\mathrm{SO}_{4}\right)_{2}$ hexahydrate, $0.25 \% \quad(\mathrm{w} / \mathrm{v}) \mathrm{CuSO}_{4}$ pentahydrate, $0.05 \% \quad(\mathrm{w} / \mathrm{v}) \quad \mathrm{MnSO}_{4}$ monohydrate, $0.05 \%$ (w/v) $\mathrm{H}_{3} \mathrm{BO}_{3}, 0.05 \%$ (w/v) $\mathrm{Na}_{2} \mathrm{MoO}_{4}$ dihydrate

Biotin stock solution: $\quad 0.01 \%(\mathrm{w} / \mathrm{v})$ biotin, $50 \%(\mathrm{v} / \mathrm{v})$ ethanol

\subsubsection{Transformation}

\section{S. cerevisiae}

Lithium Acetate (10x): $\quad 1$ M lithium acetate, $\mathrm{pH} 7.5$

TE(D) (10x): $\quad 10 \mathrm{mM}$ Tris/HCl, 1 mM EDTA, $\mathrm{pH} 7.2$

DTT: $\quad$ 1M DTT in $\mathrm{H}_{2} \mathrm{O}$

Sorbitol: $\quad 1 \mathrm{M}$ sorbitol in $\mathrm{H}_{2} \mathrm{O}$

\section{S. macrospora}

Protoplast buffer (PPB): $\quad 45 \mathrm{mM} \mathrm{KH}_{2} \mathrm{PO}_{4}, 13 \mathrm{mM} \mathrm{Na}_{2} \mathrm{HPO}_{4}, 0.6 \mathrm{M} \mathrm{KCl}$, pH 6.0

Transformation buffer (TPS): $1 \mathrm{M}$ sorbitol, $80 \mathrm{mM} \mathrm{CaCl}_{2}$, $\mathrm{pH} 7.4$ 


\subsubsection{Solutions regarding DNA}

Plasmid isolation (Birnboim \& Doly, 1979)

B\&D I: $\quad 50 \mathrm{mM}$ glucose, $25 \mathrm{mM}$ Tris/HCl, $10 \mathrm{mM}$ EDTA, 0.2\% lysozyme

B\&D II: $\quad 0.4 \mathrm{M} \mathrm{NaOH}, 2 \%(w / v)$ SDS

B\&D III: $\quad 3 \mathrm{M}$ potassium acetate, $1.8 \mathrm{M}$ formic acid

Southern Blot

Buffer I: $\quad 0.25 \mathrm{M} \mathrm{HCl}$

Buffer II: $\quad 0.5 \mathrm{M} \mathrm{NaOH}, 1.5 \mathrm{M} \mathrm{NaCl}$

Buffer III: $\quad 1.5 \mathrm{M} \mathrm{NaCl}, 0.5 \mathrm{M}$ Tris

Washing buffer I: $\quad 2 \mathrm{M}$ urea, $0.1 \%$ SDS, $50 \mathrm{mM} \mathrm{NaH}_{2} \mathrm{PO}_{4} \times \mathrm{H}_{2} \mathrm{O} \mathrm{pH} 7,150 \mathrm{mM}$

$\mathrm{NaCl}, 1 \mathrm{mM} \mathrm{MgCl} 2,0.2 \%$ blocking reagent

Washing buffer II (20x): $\quad 1 \mathrm{M}$ Tris pH 10, $2 \mathrm{M} \mathrm{NaCl}, 1 \mathrm{mM} \mathrm{MgCl} 2$

DNA-loading dye (6x): $\quad 0.25 \%(w / v)$ xylene cyanol FF, $0.25 \%(w / v)$ bromophenol blue, $40 \%(\mathrm{w} / \mathrm{v})$ sucrose

dNTP mix: $\quad 10 \mathrm{mM} \mathrm{dATP,} \mathrm{dCTP,} \mathrm{dGTP,} \mathrm{dTTP} \mathrm{separately} \mathrm{solved} \mathrm{in} \mathrm{a.} \mathrm{dest.}$

EtBr solution: $\quad 10 \mathrm{mg} / \mathrm{ml}$ ethidium bromide in $\mathrm{H}_{2} \mathrm{O}$

MOPS buffer (10x): $\quad$ 0.2 M MOPS pH 7.0, $50 \mathrm{mM}$ sodium acetate, $10 \mathrm{mM}$ EDTA

TBE (10x): $\quad 1$ M Tris/HCl, 1 M boric acid, 20 mM EDTA, pH 8.3

S.macrospora lysis buffer: $100 \mathrm{mM} \mathrm{NaCl,} 10$ mM Tris/HCl, 1 mM EDTA, 2\% SDS, pH 8.0

Yeast lysis buffer: $\quad 100 \mathrm{mM} \mathrm{NaCl}, 2 \%(\mathrm{v} / \mathrm{v})$ Triton X-100, $1 \%(w / v)$ SDS, $10 \mathrm{mM}$ Tris (pH 8.0), 1 mM EDTA

\subsubsection{Protein regarding solutions}

Coomassie:

0.02\% (w/v) coomassie brilliant blue R250, 0.02\% (w/v) coomassie brilliant blue G250, 42.5\% (v/v) ethanol 0.5\% (v/v) methanol 10\% (v/v) acetic acid

Destaining solution: $\quad 45 \%(\mathrm{v} / \mathrm{v})$ ethanol, $10 \%(\mathrm{v} / \mathrm{v})$ acetic acid 
Loading dye (5x):

Lysis buffer:

PBS (10x):

TAP buffer:

TBE (10x):

Transfer buffer:

SDS-PAGE-running buffer: $1.5 \%(\mathrm{w} / \mathrm{v})$ Tris $\mathrm{pH}$ 8.3, 9.4\% (w/v) glycine, 20\% (w/v) SDS

125 mM Tris/HCl pH 6.8, 50\% (v/v) glycerine, 2\% (w/v) SDS, $0.01 \%$ (w/v) bromophenol blue, $0.01 \%$ (w/v) $\beta$-mercaptoethanol $20 \mathrm{mM}$ imidazole, $300 \mathrm{mM} \mathrm{NaCl}, 50 \mathrm{mM} \mathrm{NaH}_{2} \mathrm{PO}_{4}$ 1.4 M NaCl, $27 \mathrm{mM} \mathrm{KCl,} 101 \mathrm{mM} \mathrm{Na}_{2} \mathrm{HPO}_{4}, 17.6 \mathrm{mM} \mathrm{KH}_{2} \mathrm{PO}_{4}$, $\mathrm{pH} 7.4$

100 mM Tris, 200-300 mM NaCl, 2 mM EDTA, 10\% glycerol, 0,1-0,5\% (v/v) Nonident ${ }^{\circledR}$ P40, 2 mM DTT, 1 mM PMSF $1 \mathrm{M}$ Tris/HCl, 1 M boric acid, 20 mM EDTA, pH 8.3 $192 \mathrm{mM}$ glycine, $25 \mathrm{mM}$ Tris, 20\% (v/v) methanol

\subsubsection{Media}

\section{E. coli}

LB: $1 \%(\mathrm{w} / \mathrm{v})$ tryptone/peptone, $0.5 \% \mathrm{NaCl}, \mathrm{pH} 7.2 ; 0.5 \%$ yeast extract, $1.5 \%(\mathrm{w} / \mathrm{v})$ agar-agar for solid medium; optional ampicillin (100 $\mu \mathrm{g} / \mathrm{ml})$ or kanamycin $(60 \mu \mathrm{g} / \mathrm{ml})$ for selection.

SOB: $2 \%(\mathrm{w} / \mathrm{v})$ tryptone, $0.5 \%(\mathrm{w} / \mathrm{v})$ yeast extract, $10 \mathrm{mM} \mathrm{MgCl}_{2}, 10 \mathrm{mM} \mathrm{MgSO}_{4}, 10 \mathrm{mM}$ $\mathrm{NaCl}, 2.5 \mathrm{mM} \mathrm{KCl}, \mathrm{pH} 7.5$.

TB: $1.86 \%(\mathrm{w} / \mathrm{v}) \mathrm{KCl}, 0.66 \%(\mathrm{w} / \mathrm{v}) \mathrm{MnCl}_{2}$ tetrahydrate, 0.3\% (w/v) HEPES, $0.22 \%(\mathrm{w} / \mathrm{v}) \mathrm{CaCl}_{2}$ dihydrate, $\mathrm{pH} 6.7$.

\section{S. cerevisiae}

YEPD: 2\% (w/v) tryptone, $2 \%(\mathrm{w} / \mathrm{v})$ glucose, $1 \%(\mathrm{w} / \mathrm{v})$ yeast extract, $\mathrm{pH} 5.8 ; 1.5 \%(\mathrm{w} / \mathrm{v})$ agaragar SERVA for solid medium.

YEPDA: YEPD + 0.003\% (w/v) adenine, $\mathrm{pH}$ 6.5; 1.5\% (w/v) agar-agar SERVA for solid medium.

SD: $0.17 \%(\mathrm{w} / \mathrm{v})$ Difco $^{\mathrm{TM}}$ Yeast Nitrogen Base w/o amino acids and ammonium sulfate, $2 \%$ $(\mathrm{w} / \mathrm{v})$ glucose, 0.064\% (w/v) CSM-Ade-His-Leu-Trp-Ura (0.002\% (w/v) L-methionine, 0.005\% (w/v) L-arginine hydrochloride, L-isoleucine, L-lysine hydrochloride, L-phenylalanine, L- 
tryptophan, L-tyrosine, uracil, each 0.008\% (w/v) L-aspartic acid, 0.01\% (w/v) L-leucine and Lthreonine, 0.014\% (w/v) L-valine), $\mathrm{pH}$ 5.8; 1.5\% (w/v) Agar-Agar SERVA for solid medium. Selection of transformants is facilitated by exclusion of respective amino acid(s).

\section{S. macrospora}

BMM: 0.8\% bio malt maize extract and maize flour (25 g/l), $\mathrm{pH} 6.5 ; 1.5 \%$ (w/v) agar-agar for solid medium; optional: addition of hygromycin B (110 U/ml) and/or nourseothricin dihydrogen sulfate $(50 \mu \mathrm{g} / \mathrm{ml})$ for selection.

BMM + sodium acetate: BMM + 0.5\% (w/v) sodium acetate (sporulation induction).

CMS: $1 \%(\mathrm{w} / \mathrm{v})$ glucose, $0.2 \%(\mathrm{w} / \mathrm{v})$ tryptone/peptone, $0.2 \%(\mathrm{w} / \mathrm{v})$ yeast extract, $0.15 \%(\mathrm{w} / \mathrm{v})$ $\mathrm{KH}_{2} \mathrm{PO}_{4}, 0.05 \%(\mathrm{w} / \mathrm{v}) \mathrm{KCl}, 0.05 \%$ (w/v) $\mathrm{MgSO}_{4}$ heptahydrate, 0.37\% (w/v) $\mathrm{NH}_{4} \mathrm{Cl}, 10.8 \%$ (w/v) sucrose, 0.01\% (v/v) trace element-stock solution (10 mg/l ZnSO $4,10 \mathrm{mg} / \mathrm{l} \mathrm{Fe}(\mathrm{II}) \mathrm{Cl}_{2}, 10$ $\mathrm{mg} / \mathrm{l} \mathrm{MnCl}_{2}$ ), $\mathrm{pH}$ 6.5; 1.5\% (w/v) agar-agar for solid medium.

$\mathrm{MM}+$ starch: $0.1 \%(\mathrm{w} / \mathrm{v})$ soluble starch, $1.8 \mathrm{mM} \mathrm{KH}_{2} \mathrm{PO}_{4}, 1.7 \mathrm{mM} \mathrm{K}_{2} \mathrm{HPO}_{4}$ trihydrate, $8.3 \mathrm{mM}$ urea, $1 \mathrm{mM} \mathrm{MgSO}_{4}$ heptahydrate, $0.01 \%$ (v/v) trace element-stock solution, $5 \mathrm{mM}$ biotin, $\mathrm{pH}$ 6.6-6.8; 1.5\% (w/v) SERVA-agar for solid medium.

SWG: 1x Westergaard's (0.1\% (w/v) $\mathrm{KNO}_{3}, \quad 0.1 \%(\mathrm{w} / \mathrm{v}) \mathrm{KH}_{2} \mathrm{PO}_{4}, \quad 0.05 \%$ (w/v) $\mathrm{MgSO}_{4}$ heptahydrate, $0.01 \%(\mathrm{w} / \mathrm{v}) \mathrm{NaCl}, 0.01 \%(\mathrm{w} / \mathrm{v}) \mathrm{CaCl}_{2}, 0.01 \%(\mathrm{v} / \mathrm{v})$ trace element-stock solution [5\% (w/v) citric acid $\left(\mathrm{C}_{6} \mathrm{H}_{8} \mathrm{O}_{7}\right.$ monohydrate) $5 \%(\mathrm{w} / \mathrm{v}) \quad \mathrm{ZnSO}_{4}$ heptahydrate, $1 \%(\mathrm{w} / \mathrm{v})$ $\mathrm{Fe}\left(\mathrm{NH}_{4}\right)_{2}\left(\mathrm{SO}_{4}\right)_{2}$ hexahydrate, $0.25 \% \quad(\mathrm{w} / \mathrm{v}) \quad \mathrm{CuSO}_{4}$ pentahydrate, $0.05 \% \quad(\mathrm{w} / \mathrm{v}) \mathrm{MnSO}_{4}$ monohydrate, $0.05 \%(\mathrm{w} / \mathrm{v}) \mathrm{H}_{3} \mathrm{BO}_{3}, 0.05 \%$ (w/v) $\mathrm{Na}_{2} \mathrm{MoO}_{4}$ dihydrate] 0.1\% (v/v) chloroform), $2 \%(\mathrm{w} / \mathrm{v})$ glucose, $0.1 \%(\mathrm{w} / \mathrm{v})$ arginine, $0.1 \%(\mathrm{v} / \mathrm{v})$ biotin-stock solution $(0.01 \%$ in $50 \%$ ethanol), $\mathrm{pH}$ 6.5; 1.5\% (w/v) agar-agar for solid medium; addition of hygromycin B (110 U/ml) or nourseothricin dihydrogen sulfate $(50 \mu \mathrm{g} / \mathrm{ml})$ for selection. 


\subsection{Strains and culture conditions}

All strains used in this study are enlisted in Table 2.

E. coli Mach1 strain was used for amplification of generated plasmids. The respective strains were grown in liquid LB medium at $37^{\circ} \mathrm{C}$ and $200 \mathrm{rpm}$, supplemented with antibiotics depending on the amplified plasmid.

S. cerevisiae strains were inoculated in liquid or on solid $\operatorname{YEPD(A)}$ or SD minimal medium supplemented with appropriate aa for selection. Strains were incubated at $30^{\circ} \mathrm{C}$. Cultures in liquid medium were shaken at 150 rpm (Amberg et al., 2005).

S. macrospora was grown on BMM, complete medium containing 10.8\% saccharose (CMS) and fruiting-body development inducing SWG medium at $27^{\circ} \mathrm{C}$. For DNA extraction, transformation or protein extraction, S. macrospora strains were grown in liquid medium containing petri dishes (Nowrousian \& Cebula, 2005).

Basic methods have been conducted according to Sambrook et al. (2001) and were not separately described.

\subsubsection{Preparation and transformation procedures}

\section{E. coli}

For preparation of chemo-competent E. coli cells, strain Mach1 was grown in liquid SOB medium at 20-30 rpm to an $\mathrm{OD}_{600}$ of 0.6 . Subsequently, cells were incubated on ice for $10 \mathrm{~min}$ and harvested by centrifugation at $2500 \mathrm{~g}$ at $4^{\circ} \mathrm{C}$. The cell pellet was resuspended in TB and incubated for additional $10 \mathrm{~min}$ on ice, followed by a centrifugation step at $2500 \mathrm{~g}$ for $10 \mathrm{~min}$ at $4^{\circ} \mathrm{C}$. Afterwards the cell pellet was resuspended in TB and DMSO, incubated for 10 min on ice, aliquoted in $150 \mu \mathrm{l}$ samples and frozen in liquid nitrogen. Cells were stored at $-80^{\circ} \mathrm{C}$. Transformation was done according to Sambrook et al. (2001). For transformation, cells were thawed at RT, mixed with plasmid DNA and incubated on ice for $20 \mathrm{~min}$, followed by an incubation for $1 \mathrm{~min}$ at $42^{\circ} \mathrm{C}$ and subsequently again for $10 \mathrm{~min}$ on ice. Then, liquid LB was added to the cells and the samples were incubated for $20-40 \mathrm{~min}$ at $37^{\circ} \mathrm{C}$ at $200 \mathrm{rpm}$. Finally, the cells were transferred to LB medium supplemented with respective antibiotics. 


\section{S. cerevisiae}

For preparation of electro-competent $S$. cerevisiae cells, yeast strains were grown to an $\mathrm{OD}_{600}$ of 1.0-1.2 in YPD medium, centrifuged for $5 \mathrm{~min}$ at $8000 \mathrm{~g}$ and resuspended in a $\mathrm{LiAc} / \mathrm{TE}(\mathrm{D}) / \mathrm{H}_{2} \mathrm{O}$ solution in the ratio $1: 1: 8$. This step was followed by incubation at $30^{\circ} \mathrm{C}$ on a shaker with 100 rpm. After $45 \mathrm{~min}$, DDT was added, and the incubation was prolonged for additional $10 \mathrm{~min}$. Cells were harvested by centrifugation, washed once with $\mathrm{H}_{2} \mathrm{O}$ and once with $1 \mathrm{M}$ sorbitol. After final uptake in $1 \mathrm{M}$ sorbitol, transformation was carried out by electroporation using an Eppendorf Electroporator 2510 (Eppendorf) with $1.5 \mathrm{kV}$. Samples were mixed as followed: $40 \mu \mathrm{l}$ of competent cells and the respective DNA fragments were added to an electroporation cuvette, shocked and resuspended in $600 \mu \mathrm{l}$ sorbitol and plated on solid SD medium lacking the respective aa for selection (Becker \& Lundblad, 2001).

\section{S. macrospora}

Protoplastation of S. macrospora strains was achieved according to Pöggeler et al. (1997). Cultures of S. macrospora were grown for 3-4 days in petri dishes as described above. Strains were harvested, transferred to PPB containing $20 \mathrm{mg} / \mathrm{ml}$ natuzym and incubated for 3 hours at $27^{\circ} \mathrm{C}$ and $125 \mathrm{rpm}$. Protoplasts were isolated using a frit (ROBU glass, pore size 1) into a $50 \mathrm{ml}$ tube. Protoplasts were washed twice with 10 and $5 \mathrm{ml}$ PPB by centrifugation at $4.400 \mathrm{rpm}$ for 5.5 min. Protoplasts were resuspended in $100 \mu \mathrm{l}$ TPS. For each transformation sample, $100 \mu 1$ protoplasts were added to $20 \mu \mathrm{g}$ DNA and incubated on ice for $10 \mathrm{~min}$. A PEG6000 solution (25\% [w/v] PEG6000 in PPB) was added to the transformation samples and gently mixed by inverting the reaction tube. After 20 min incubation at RT, the protoplast suspension was spread on solid CSM medium and incubated for $24 \mathrm{~h}$ at $27^{\circ} \mathrm{C}$. Regenerated protoplasts were covered with hygromycin or nourseothricin supplemented top-agar $(4.7 \%[\mathrm{w} / \mathrm{v}] \mathrm{NaCl}$ and $0.8 \%$ [w/v] SERVA-agar) for selection. The final concentration of antibiotics was $50 \mu \mathrm{g} / \mathrm{ml}$ nourseothricin dihydrogen sulfate or/and $110 \mathrm{U} / \mathrm{ml}$ hygromycin B. 


\subsubsection{DNA methods}

\subsubsection{Plasmid isolation from $E$. coli and $S$. cerevisiae}

\section{E. coli}

Plasmids were isolated using either the QIAprep Spin Miniprep Kit, the HiSpeed Plasmid Midi Kit or the plasmid extraction protocol according to Birnboim and Doly (Birnboim \& Doly, 1979). Plasmid extraction kits were used according to the manufacturer's protocol. Plasmid extraction protocol according to Birnboim \& Doly (1979) was applied to $50 \mathrm{ml}$ LB E. coli cultures. Cells were grown over night at $37^{\circ} \mathrm{C}$ at $200 \mathrm{rpm}$ and harvested by centrifugation at $4000 \mathrm{~g}$ for $15 \mathrm{~min}$. The cell pellet was resuspended in $2 \mathrm{ml}$ B\&D I solution containing $10 \mu \mathrm{g}$ freshly added RNase and incubated at RT for $5 \mathrm{~min}$. Afterwards $2 \mathrm{ml} \mathrm{B \& D}$ II solution were added and the sapmle mixed by vortexing and incubated for $5 \mathrm{~min}$ at RT. After addition of $2 \mathrm{ml}$ B\&D III solution, the sample was mixed by inverting the tube and centrifuged for 15 min at max rpm. The supernatant was transferred to a new tube and mixed with $7.5 \mathrm{ml}$ isopropanol and incubated for $20 \mathrm{~min}$ at $-20^{\circ} \mathrm{C}$ for DNA precipitation. The precipitated DNA was collected by centrifugation and washed with $5 \mathrm{ml}$ of $70 \%$ ethanol. The residual ethanol was removed and the pellet was air dried. The DNA pellet was resuspended in 1-2 $\mathrm{ml}$ a. dest depending on its concentration.

\section{S. cerevisiae}

Plasmids were isolated using either the QIAprep Spin Miniprep Kit or the Smash and Grab protocol according to Hoffman and Winston (1987). Plasmid extraction kits were used according to the manufacturer's protocol with minor changes. Additionally $0.3 \mathrm{~g}$ of glass beads $(\varnothing 0.25-0.5$ $\mathrm{mm}$ ) were added to the P1 buffer in the tube to disrupted cell walls mechanically by vortexing. The Smash and Grab protocol was applied to yeast cultures grown $<16$ h in 5-20 ml liquid medium and harvested by centrifugation for $3 \mathrm{~min}$ at 8000-12000 g. The cell pellet was resuspended in 0.5-1 $\mathrm{ml} \mathrm{H} \mathrm{H}_{2} \mathrm{O}$ and centrifuged again at $12000 \mathrm{~g}$ for $2 \mathrm{~min}$. The supernatant was discarded and the cell pellet resuspended in residual liquid through vortexing. $200 \mu$ lysis buffer, $200 \mu \mathrm{l}$ phenol/chloroform solution and $0.3 \mathrm{~g}$ of glass beads ( $\varnothing 0.25-0.5 \mathrm{~mm})$ were added and the sample was vortexed in a thermomixer (Eppendorf) for $8 \mathrm{~min}$ at $1,400 \mathrm{rpm}$ at $4^{\circ} \mathrm{C}$. The content was centrifuged for $15 \mathrm{~min}$ at $12000 \mathrm{~g}$. Subsequently, $250 \mu \mathrm{l}$ of the upper, aqueous layer were 
transferred in a fresh reaction tube. DNA was precipitated with isopropanol to remove residual phenol.

\subsubsection{Isolation of RNA and genomic DNA from S. macrospora}

RNA isolation: RNA isolation was done according to Elleuche and Pöggeler (2009) with minor changes. The mycilium was harvested, dried and grounded in liquid nitrogen. About $1 \mathrm{~g}$ of powdered mycelium was mixed with $1 \mathrm{ml}$ Trizol and centrifuged for $10 \mathrm{~min}$ at $13000 \mathrm{rpm}$ and $4^{\circ} \mathrm{C}$. The supernatant was transferred to a new tube and mixed with $200 \mu \mathrm{l}$ chloroform. After centrifugation, the chloroform-generated upper phase was transferred to a new tube mixed with the same amount of isopropanol and after $10 \mathrm{~min}$ of incubation at RT centrifuged for $10 \mathrm{~min}$ at $13000 \mathrm{rpm}$ and $4^{\circ} \mathrm{C}$. The obtained pellet was dried under an outlet and resuspended in $120 \mu 1$ RNA free $\mathrm{H}_{2} \mathrm{O}$. Finally, the sample was incubated for $30 \mathrm{~min}$ at $1000 \mathrm{rpm}$ and $60^{\circ} \mathrm{C}$ in a thermomixer (Eppendorf).

DNA extraction: gDNA isolation was done using phenol/chloroform extraction according to Lecellier and Silar (1994). The mycelium was harvested, transferred to a $2.0 \mathrm{ml}$ reaction tube and frozen at $-80^{\circ} \mathrm{C}$ for $30 \mathrm{~min}$. The frozen pellet was taken up in $600 \mu \mathrm{S}$. macrospora lysis buffer. 8-10 glass beads ( $\varnothing 2.85-34.45 \mathrm{~mm}$ ) were added to the tube to (finally) mechanically disrupt the cell wall in a TissueLyser (Eppendorf) for $5 \mathrm{~min}$ at $30 \mathrm{~Hz}$. The sample was mixed with $500 \mu \mathrm{l}$ phenol, briefly vortexed and centrifuged at $13000 \mathrm{rpm}$ for $10 \mathrm{~min}$. The upper (aqueous) phase was transferred to another $2.0 \mathrm{ml}$ reaction tube and mixed with $1 \mathrm{ml}$ phenol/chloroform by vortexing. After another centrifugation step, the aqueous layer was transferred to a new $2.0 \mathrm{ml}$ reaction tube and mixed with $1 \mathrm{ml}$ chloroform followed by centrifugation. Again, the aqueous layer was transferred into a $1.5 \mathrm{ml}$ reaction tube. The DNA was precipitated by adding isopropanol and incubation for $15 \mathrm{~min}$ at RT. After a final centrifugation step, the DNA pellet was dried under an outlet and resuspended in an appropriate amount of $\mathrm{H}_{2} \mathrm{O}$.

\subsubsection{PCR}

Amplification of S. macrospora cDNA and gDNA as well as verification of strains via PCR or colony PCR on E. coli or S. cerevisiae plasmid DNA was achieved with HotStarTaq Master Mix Kit (Qiagen), Molzyme MolTaq polymerase (Molzym GmbH \& Co. KG), Pfu polymerase 
(Promega GmbH) or Phusion High-Fidelity DNA polymerase (New England Biolabs). All polymerases were used according to the manufacturer's protocol.

\subsubsection{Purification of amplified DNA}

\section{Gel extraction}

DNA extraction from agarose gels was done using the QIAquick Gel Extraction Kit (Qiagen) according to the manufacture's protocol.

\section{Micro-dialysis membranes}

Dialysis of nucleic acids after PCR amplification was conducted to get rid of salts present in reaction buffers. Nucleic acids were pipetted on membranes (Millipore, $0.02 \mu \mathrm{m}$ pore size) floating on a. dest. in a petri dish and incubated for $10 \mathrm{~min}$ at RT. The desalted DNA was removed from the membranes via pipetting and used for further experiments.

\subsubsection{5 cDNA synthesis}

To ensure that the template RNA contained no remaining gDNA, it was treated with DNaseI according to the manufacturer's protocol. Reverse transcription reaction was done with Transcriptor High Fidelity cDNA Synthesis kit according to the manufacturer's recommendations. $2 \mu \mathrm{g}$ of RNA were conducted as template for reverse transcriptase reaction. Each reaction was made twice, once with and once without reverse transcriptase. The sample without template served after RNase treatment as control for final exclusion of gDNA remnants by PCR.

\subsubsection{Hydrolysis of nucleic acids.}

DNA for southern blot analysis, ligation after gel extraction or plasmid verification was done by hydrolysis with restriction enzymes according to the manufacturer's protocol. The sample size depended on further use or experimental setup (generally ranging from 5-75 $\mu \mathrm{l}$ ).

\subsubsection{Ligation of DNA fragments}

Fragments were ligated into the respective vectors using either QIAGEN PCR Cloning Kit (Qiagen), T4 ligase (Thermo Scientific) or In-Fusion® HD Cloning Kit (Clontech). QIAGEN 
PCR Cloning Kit and Clontech In-Fusion ${ }^{\circledR}$ HD Cloning Kit were used according to the manufacture's recommendations.

T4 ligase reactions were set up as follows: $1 \mu \mathrm{T} 4$ ligase, $2 \mu \mathrm{l} 10 \mathrm{x}$ ligation buffer, 1-2 $\mu \mathrm{l}$ vector DNA and 15-16 $\mu$ dialyzed PCR fragment. The sample was incubated at 30 or $37^{\circ} \mathrm{C}$ for $30 \mathrm{~min}$ or overnight at $16^{\circ} \mathrm{C}$. Before ligation with $\mathrm{T} 4$ ligase, the hydrolyzed vectors were dephosphorylated with calf intestine alkaline phosphatase (CIAP, Thermo Scientific). The general setup contained $2.5 \mu \mathrm{l}$ 10x CIAP buffer, $1 \mu \mathrm{l}$ CIAP and $21.5 \mu \mathrm{l}$ vector DNA, with incubation at $37^{\circ} \mathrm{C}$ for $10 \mathrm{~min}$ followed by $10 \mathrm{~min}$ at $75^{\circ} \mathrm{C}$ to inactivate the enzyme.

\subsubsection{Separation of nucleic acids by gel electrophoresis}

\section{DNA}

DNA fragments contained after PCR or digestion were mixed with 6x loading dye and separated in $0.8-1 \%$ agarose gels $(1 \%[\mathrm{w} / \mathrm{v}]$ agarose in $1 \mathrm{x}$ TBE buffer) in a gel electrophoresis chamber at a voltage of 50-135 V. 0.5x TBE buffer was used as electrophoresis buffer. The gel, containing the separated DNA was incubation in a $1 \mu \mathrm{g} / \mathrm{ml}$ ethidium bromide solution for $10-20 \mathrm{~min}$ and visualized under UV light.

\section{RNA}

RNA fragments were separated in agarose gels containing 1.2\% 1x MOPS and 5\% formaldehyde at 80-100 V in a gel electrophoresis chamber. Prior, the respective RNA was mixed in a ratio 1:1 with 2x RNA loading dye already containing ethidium bromide and incubated for $10 \mathrm{~min}$ at $65^{\circ} \mathrm{C} .1 \mathrm{x}$ MOPS was used as electrophoresis buffer. Similar to DNA, RNA was visualized under UV light, Gene Ruler DNA Ladder Mix served as marker.

\subsubsection{Southern blotting}

Total gDNA of the respective strains for Southern blotting was isolated as described above. Depending on the final concentrations, about $25 \mu \mathrm{l}$ of the obtained gDNA was hydrolyzed and separated by gel electrophoresis. The gel was washed in buffer I $(0.25 \mathrm{M} \mathrm{HCl})$ for $10 \mathrm{~min}$, in buffer II ( $0.5 \mathrm{M} \mathrm{NaOH})$ for $25 \mathrm{~min}$ and finally in buffer III (1.5 M NaCl, 0.5 Tris, pH 7.4) for 30 min. The DNA was transferred to a GeneScreen Hybridization Transfer Membrane (PerkinElmer) by capillary blotting technique and cross-linked to the membrane via exposure to 
UV light for 5 min. DNA-DNA hybridization and detection reaction were done with High Prime DNA Labeling and Detection Starter Kit II (Roche) according to the manufacturer's protocol. After adding the detection agent to the membrane, the X-ray films were exposed for a minimum of 30 min to a maximum of 6 hours.

\subsubsection{Protein methods}

\subsubsection{S. macrospora protein extraction}

S. macrospora strains were grown in liquid $\mathrm{BMM}$ or SWG medium at $27^{\circ} \mathrm{C}$ for $3-6$ days, optional with additives hygromycin $\mathrm{B}(80 \mathrm{U} / \mathrm{ml})$ and/or nourseothricin dihydrogen sulfate (35 $\mu \mathrm{g} / \mathrm{ml})$. The mycelium was harvested, dried and grounded in liquid nitrogen. $560 \mu \mathrm{l}$ protein buffer were added to $1 \mathrm{~g}$ pulverized mycelium. The samples were centrifuged for $15 \mathrm{~min}$ at 8000-12000 g (depending on the sample size), the supernatant was transferred into a new tube, mixed with an appropriate amount of loading dye and heated up for 5-10 min at $95^{\circ} \mathrm{C}$ (Laemmli, 1970). For analysis of protein secretion, liquid medium was filtered with filter papers (Sartorius FT-3-303-185, $3 \mathrm{hw}$ ) and proteins were concentrated using Spin-X UF concentrator (Corning, Germany).

\subsubsection{Protein concentration measurement}

Protein concentrations were measured by a Bradford assay (Bradford 1976). Prior to adding loading dye to the protein extracts, $10 \mu \mathrm{l}$ of the extraction was mixed with $990 \mu \mathrm{l}$ of Bradford reagent and incubated for $5 \mathrm{~min}$ at RT. The concentration was measured at $595 \mathrm{~nm}$ with a Libra S12 (biochrom, UK) spectrophotometer in $1 \mathrm{ml}$ cuvettes.

\subsubsection{Immuno Blotting}

For detection of proteins S. macrospora protein extracts were separated in 10-15\% SDS-PAGE and transferred onto a PVDF or nitrocellulose membrane by Western blot (Bloemendal et al., 2012, Laemmli, 1970, Towbin et al., 1979). For immunodetection monoclonal mouse anti-eGFP antibody (Santa Cruz Biotechnology, sc-9996, 1:4000), anti-FLAG (Sigma-Aldrich, F3165, 1:12000), anti-HA (Sigma-Aldrich, H9658, 1:3000) or monoclonal anti-Actin antibody (Novus Biochemicals, NB100-74340, 1:2000) combined with a secondary HRP-linked goat anti-mouse antibody (Dianova, 115-035-003, 1:10000) were used (except for eGFP-antibody, this one is 
already labeled). Signals were detected by the Immobilon Western Kit (Millipore, WBKLS0500). Membranes were stripped in a solution of 0.2\% Ponceau S dissolved in 3\% TCA for $1 \mathrm{~h}$ at RT and washed with 5\% skim milk containing PBS or TBS.

\subsubsection{Yeast Two-Hybrid studies}

Two hybrid analysis in S. cerevisiae was conducted by using Matchmaker two-hybrid system 3 (Clontech). For protein interaction studies in S. cerevisiae, strain MAT $\alpha$ Y187 was transformed with plasmids pBD-SmGPI1 aa 1-253, pBD-SmGPI1 aa 28-227, pBD-SmGPI1 aa 1-100, pBDSmGPI1 aa 101-253 and pBD11 aa 282-845, pBD-SmKIN3 or pBD-SmKIN24. To generate pBD-SmGPI1 aa 1-253, Smgpi1 was amplified from cDNA with primer Sm9375_Y2H_ges_f and Sm9375_Y2H_ges_r, subcloned in vector pJET (Thermo Scientific K1232), hydrolyzed with NdeI and EcoRI and ligated into vector pGBKT7. Similarly, plasmid pBD-SmGPI1 aa 28-227 was generated by using primers Sm9375_Y2H_oSigseq_f and Sm9375_Y2H_oSigseq_2r. Plasmids pBD-SmGPI1 aa 1-100 and pBD-SmGPI1 aa 101-253 were constructed by using primer Sm9375_Y2H_ges_f and Sm9375_Y2H_5'_2r or Sm9375_Y2H_3'ff and Sm9375_Y2H_ges_r, respectively. pGBKT7 constructs were transformed into yeast strain Y187. pBD-SmKIN3 or pBD-SmKIN24 were generated by using In-Fusion HD Cloning Kit (Clontech). Smkin3 was amplified from cDNA with primers kin3_pBD_inf_F and kin3_pBD_inf_R, Smkin24 was amplified from cDNA with primers kin24_pBD_inf_F and kin24_pBD_inf_R and inserted into vector pGBKT7. Yeast strain MATa AH109 was transformed with pAD-SmMOB3, pAD-SmMOB3 aa 1-144 or pAD11FL. For construction of plasmid pAD-SmMOB3, Smmob3 was amplified from cDNA using primer Mob3_Y2H_f and Mob3_Y2H_R. Plasmid pAD-SmMOB3 aa 1-144 was generated by ligation of Smmob3 cDNA amplified with primers Mob3_Y2H_f and Mob3_as1-144_r into pGADT7. pAD11FL was constructed by ligation of pro11 full-length cDNA amplified with primers Pro11_Y2H_F and Pro11_Y2H_R into pGADT7. The respective Y187 and AH109 strains were mated as described previously (Bendixen et al., 1994) and selected on solid SD medium lacking leucine (leu) and tryptophan (trp). Positive colonies from SD medium without leu and trp were used for drop plate assays. A serial dilution of cells was spread on SD agar plates without leu, trp and adenine (ade). To prove whether the respective genes in vector pGBKT7 were expressed properly, a test based 
on RanBPM was used (Tucker et al., 2009). Each construct was additionally checked for selfactivation as described by Jacobsen et al. (2002).

\subsubsection{Co-IP}

Co-Immunoprecitation was conducted as described previously by Bloemendal et al. (2012) with minor changes. Interaction studies of SmKIN3 and PRO11 were done with additional use of thiol cross-linking reagent bismaleimidohexane BHM (Thermo Scientific, 22330) solved in DMSO at a concentration of $20 \mathrm{mM}$. The cross-linker was used in a final concentration of $0.2 \mathrm{mM}$. The reaction was stopped after incubation for $2 \mathrm{~h}$ at $4^{\circ} \mathrm{C}$ by adding DTT in a final concentration of 25 $\mathrm{mM}$ as recommended in the manufacturers protocol.

\subsubsection{Differential centrifugation}

Pulverized mycelium was mixed with extraction buffer and centrifuged at $3000 \mathrm{~g}$ for $3 \mathrm{~min}$. The obtained crude extract was centrifuged for additional $15 \mathrm{~min}$ at $8000 \mathrm{~g}$. The remaining pellet was used as sample containing cell detritus, including the cell wall. An aliquot of the supernatant of this sample served as crude extract. The remaining supernatant was centrifuged for $1 \mathrm{~h}$ at $4000 \mathrm{~g}$ followed by an ultracentrifugation step for $1.5 \mathrm{~h}$ at $90000 \mathrm{~g}$. The supernatant containing the cytosol obtained by ultracentrifugation as well as the pellet containing membranes were both used as sample for distribution analysis.

\subsubsection{Crossbreeding of S. macrospora}

The respective S. macrospora strains were crossed, usually with a spore-color mutant, by inoculating them directly towards each other on a petri dish with solid SWG medium. The plates were incubated for $10-12$ days at $27^{\circ} \mathrm{C}$ until the crossing front was formed in the middle of the petri dish. This crossing front represents the genetic assembly of the crossed strains and thus, contained the recombinant crossing perithecia. In case of using spore-color mutant, these contained spores with 2 colors in a typical pattern based on segregation (Esser, 1992). To obtain single spore isolates crossing perithecia were broken on preparation agar ( $5 \%$ agar-agar in $\mathrm{H}_{2} \mathrm{O}$ ). The spores were separated and spread on solid BMM sodium acetate medium optionally supplemented with hygromycin B $(110 \mathrm{U} / \mathrm{ml})$ or nourseothricin dihydrogen sulfate $(50 \mu \mathrm{g} / \mathrm{ml})$ for selection. 


\subsubsection{Generation of $S$. macrospora single-knockout strains}

For generation of the $\Delta$ Smkin3, $\Delta$ Smkin24 and $\Delta$ Sm3978 single-deletion strains, 5' flanking regions and 3' flanking regions of the respective genes were fused to the hph hygromycin resistance cassette and integrated into plasmid backbone pRSnat (Klix et al., 2010) by homologous recombination in the yeast strain PJ69-4A (Colot et al., 2006). 5‘ regions of Smkin3, Smkin24 and Sm3978 were amplified with primer combinations Smkin3_1k_5F x Smkin3_5R, Smkin24_1k_5F x Smkin24_5R and 3978_5F x 3978_5R. The 3' flanking regions of Smkin3, Smkin24 and Sm3978 were amplified with primer pairs Smkin3_3F x Smkin3_1k_3R, Smkin24_3F x Smkin24_1k_3R or Sm3978_3F x Sm3978_3R. Each amplified flanking region had an overhang to hph amplified with primers hph_F x hph_R from plasmid pCB1003 (Carroll et al., 1994). This resulted in deletion constructs consisting of the $h p h$ resistance cassette surrounded by the 5' and the 3' flanking region of the gene of interest. After homologous recombination in yeast, the plasmids were isolated and the deletion constructs were amplified from the respective plasmids, purified and transformed into S. macrospora strain $\Delta$ ku 70 (Pöggeler \& Kück, 2006). The $\Delta$ ku70 background (nourseothricin resistance) was eliminated by crossing primary transformants to spore-color mutant r2 (Teichert et al., 2014). Hygromycin resistant, nourseothricin sensitive single-spore isolates were selected. The constructed single deletion strains were verified by PCR and Southern blot. For PCR verification, gDNA was isolated and presence of the deletion cassette at the correct gene locus was ensured by amplification with primers binding upstream of the 5' region or downstream of the 3' region and within the hph resistance cassette. Thus, primers binding outside of the flanking regions used for the deletion cassette and within the $h p h$ gene verified that the gene of interest was disrupted by the $h p h$ gene. Any deletion cassette was amplified in total with primers binding outside the flanking regions or each flanking region in particular. The complementary primers for flankingregion amplification bound within the $h p h$ cassette. The amplicons obtained from the knockout strains were compared to amplicons with the same primer combinations from wt. Insertion of multiple deletion constructs at various locations was excluded by southern blot. 


\subsubsection{Generation of $S$. macrospora double-knockout strains}

Double-deletion strains were obtained by crossing the respective single-deletion strains as described above. Usually, at least one of the single deletion strains used for crossing was previously crossed with a spore-color mutant to identify crossing perithecia easily. After crossing perithecia were broken, entire asci were separated and all 8 included spores were spread on solid BMM sodium acetate medium. After germination, the isolated single spore isolates were verified by PCR as described for single-knockout strains and via southern blot.

\subsubsection{Generation of $S$. macrospora complementation strains}

To verify whether observed phenotypes were based on the respective gene deletion, each deleted gene was inserted ectopically in the genome of the respective single- or double-knockout strain by transformation. The complementation constructs were generated by using the forward 5' flanking region and the reverse 3' flanking region primer for PCR on wt gDNA. This resulted in amplicons, equal in flanking regions to the deletion constructs but also containing the ORF of the deleted genes. Similar to gene deletion, integration of the complementation constructs was verified by PCR. The obtained, genetically complemented strains were subsequently phenotypically analyzed for phenotypic complementation. By this, phenotypes could be dedicated to gene deletions. Additionally, eGFP-constructs were checked for complementation and thus to be functional.

\subsubsection{Analytic procedures}

\subsubsection{Light and fluorescence microscopy investigations}

To localize SmGPI1 and to verify the predicted signal sequence and the region for GPI-anchor attachment, several eGFP constructs were generated. Plasmid pRS-SmGPI1-eGFP aa 1-492 represents the eGFP-tagged full-length construct of SmGPI1. To generate pRS-SmGPI1-eGFP aa 1-492, the sequence encompassing bp -967 to 869 was amplified using primer Gpi1_5_inf_F2 and Core-gfp_inf_R2. In the next step, egfp was amplified with GFP_f and GFP_r from plasmid p1783-1 (Pöggeler et al., 2003) and the region for GPI-anchor attachment encompassing bp 870980 using primer pair Omega-gfp_inf_F and 9375_o_r. Finally, the trpC terminator of A. nidulans was amplified from plasmid p1783-1 using primer TrpC_F and pRS426GFPrev. All 
fragments were ligated using In-Fusion HD Cloning Kit into pRSnat in the same order, as listed. This resulted in plasmid pRS-SmGPI1-eGFP aa 1-492 encoding for an eGFP-tagged version of SmGPI1 consisting of aa 1-217 of SmGPI1 fused to eGFP, fused to the region for GPI-anchor attachment from $\omega$-10 to the C-terminal end of SmGPI1 under control of the native promoter and the $\operatorname{trp} C$ terminator. For construction of pRS-SmGPI1-eGFP aa 1-466 Smgpi1 was amplified with primer Gpi1_5_inf_F2 and Core-gfp_inf_R2, egfp. The native promoter and the trpC terminator were amplified as described above. These fragments were ligated into pRSnat resulting in a plasmid encoding SmGPI1 aa 1-227 fused to eGFP under control of its native promoter and the trpC terminator. Plasmid pRS-SmGPI1-eGFP aa 28-492 encodes eGFP-tagged SmGPI1 without the signal sequence at position aa 1-27 and was generated by amplifying the promoter of Smgpi1 separately with primer pair Gpi1_5_inf_F2 and 5utr-GPI1oh-r. Fragments consisting of bp 82-900 of the Smgpi1 ORF, egfp, the putative omega region and the $\operatorname{trpC}$ terminator were amplified from pRS-SmGPI1_pre aa 1-492 using Omega-gfp_inf_F and pRS426GFPrev. Plasmid pRS-SmGPI1-eGFP aa 28-466 lacking N-terminal and C-terminal processing sites was generated by ligation of amplified fragments from pRS-SmGPI1-eGFP aa 28-492 with primer pair Gpi1_5_inf_F2 and Core-gfp_inf_R2 to the trpC terminator amplified from pRS-SmGPI1-eGFP aa 1-466 with primer GFP_F and pRS426GFPrev. To localize SmKIN3 and SmKIN24 in S. macrospora, N-terminally eGFP tagged constructs were generated and traced in the respective deletion strain. Plasmids coding for SmKIN3-eGFP and SmKIN24eGFP were constructed by amplifying the full length genes Smkin3 or Smkin24 and inserting them into NotI digested pDS23-egfp vector (Teichert et al., 2012) via homologous recombination in yeast. For light and fluorescence microscopic analysis, S. macrospora strains were inoculated on solid BMM medium slightly overlapping to a piece of cellophane at $27^{\circ} \mathrm{C}$ for 1 or 2 days or were grown on BMM covered glass slides. For microscopy, the cellophane sheet with the mycelium was transferred to a glass slide. Glass slides with cellophane or BMM were covered with water and a cover slip for microscopic analysis. For the visualization of hyphae or sexually developed structures, an AxioImager M1 microscope (Zeiss, distributed by Visitron Systems $\mathrm{GmbH}$ ) combined with a Photometrics CoolSNAP 2 HQ camera (Roper Scientific, Photometrics) was used. The obtained pictures were processed with Metamorph (version 6.3.1; Universal Imaging) and GIMP 2.8.2. (GNU Image Manipulation Program, The GIMP Development Team) as well as Illustrator CS2 (Adobe). To display eGFP, DsRED or DAPI fluorescence chroma filter 
set 49002, 49005 and 49000 were used, respectively. DAPI staining was conducted by adding 20-50 $\mu$ l DAPI (AppliChem, A1001,0010), dissolved in fixing solution (3.7\% formaldehyde, 0.2\% Triton X-100, $50 \mathrm{mM}$ phosphate buffer $\mathrm{pH}$ 7.0, $1 \mathrm{M} \mathrm{K}_{2} \mathrm{HPO}_{4}, 1 \mathrm{M} \mathrm{KH}_{2} \mathrm{PO}_{4}$, diluted 1:1 with $\mathrm{H}_{2} \mathrm{O}$ ) directly on the mycelium. Staining with FM 4-64 (Invitrogen, F34653) was performed by adding 20-50 $\mu \mathrm{l}$ of a FM 4-64 (1 $\mu \mathrm{g} / \mathrm{ml}$ a. dest.) solution similar to DAPI staining. MitoTracker Red (Life Technologies, M22425) was used in a concentration of $25 \mathrm{nM}$ diluted in DMSO according to the guidelines of the manufacturer. Calcofluor white staining was conducted by adding $40 \mu \mathrm{l}$ calcofluor white (Sigma-Aldrich, 18909), freshly diluted 1:1 with $10 \% \mathrm{KOH}$ solution and directly applied on the mycelium.

\subsubsection{Sequence analysis and oligonucleotide synthesis}

Primers used in this study were purchased at MWG Biotech AG and are listed in Tab 3. DNA sequencing was done by the G2L-sequencing service of the "Göttinger Genom Labor" (GeorgAugust-University Göttingen). Molecular weights, isoelectric points of proteins and domain predictions were done with programs from the ExPASy Proteomics Server (http://www.expasy.org). Protein sequence alignments were performed using the ClustalX2 program as described by Larkin et al. (2007) and visualized using GeneDoc (Nicholas et al., 1997). Protein and nucleotide sequences were obtained from the public databases at NCBI (http://www.ncbi.nlm.nih.gov/entrez/) or by BLAST searches of the complete sequenced genomes at the Broad Institute (http://www.broad.mit.edu/annotation/fungi/fgi/).

\subsubsection{Phylogenetic analysis}

Multiple protein sequence alignments were performed using the ClustalX2 program (Larkin et al., 2007). Phylogenetic analysis were made with programs from package PHYLIP version 3.6 (http://evolution.genetics.washington.edu/phylip.html). Distance matrices were calculated using the program PRODIST and afterwards used for constructing phylogenetic trees with the neighbor-joining (NJ) program NEIGHBOR. To evaluate the statistical significance a bootstrap analysis with 1000 iterations of bootstrap samplings and reconstruction of trees was performed. A majority rule consensus tree was subsequently generated with the program CONSENSE, viewed using the program TreeView 1.6.6 (Page, 1996) and saved for graphical representation using Adobe Illustrator. 


\subsubsection{Measures of safety}

Genetic engineering experiments of security level 1 have been conducted according to the guide lines of the genetic engineering law (GenTG) stated on 16.12.1993 (recently altered by Art. 42 Abs. $1 \mathrm{G}$ on 09.12. 2010). 


\section{Results}

\subsection{The GPI-anchored protein SmGPI1}

\subsubsection{A two-hybrid screen identified a GPI-anchored protein as an interaction partner of STRIPAK SmMOB3}

S. macrospora is more closely related to $N$. crassa than any other previously sequenced filamentous fungus, with $90 \%$ nucleic acid identity in coding regions of orthologous genes (Nowrousian, 2010, Nowrousian et al., 2004). Previously, cross-species microarrays with S. macrospora cDNA hybridized on $N$. crassa microarrays have been performed successfully (Nowrousian et al., 2005, Pöggeler et al., 2006). To identify new interaction partners of the STRIPAK protein SmMOB3, we performed cross-species yeast two-hybrid (Y2H) screens with a Matchmaker Two-Hybrid System 3 (Clontech) and an N. crassa cDNA library (S. Seiler, pers. comm). Using Smmob3 cDNA as bait, the $N$. crassa NCU09375 protein was identified as an interaction partner of SmMOB3. A BLASTP search of the S. macrospora proteome identified SMAC_12074 as NCU09375 homolog. The ORF of SMAC_12074 (F7W197) is 980 bp with two introns of 96 and 122 bp at positions 285-380 and 677-798, respectively. Intron splicing was verified by cDNA sequencing (data not shown). The calculated molecular mass of the 253 aa protein SMAC_12074 is 26 kDA with an isoelectric point of 5. In silico analysis using SignalP

(Petersen et al., 2011) revealed a signal sequence at aa 1-27. In addition, a region for posttranslational modification with a GPI-anchor was identified using big-PI Predictor (Eisenhaber et al., 1998, Eisenhaber et al., 1999, Eisenhaber et al., 2000, Sunyaev et al., 1999). The GPI-anchor is linked to a C-terminal residue after a proteolytic cleavage at the $\omega$-residue. In SMAC_12074, the $\omega$-residue is predicted to be Asn228. The surrounding region of the predicted $\omega$-residue has the general features of GPI-anchored proteins such as an upstream linker region, characterized by a low amount of predicted secondary structures, small side-chain residues at positions $\omega-1$ to $\omega+2$, a spacer region between positions $\omega+3$ and $\omega+9$; and a hydrophobic tail from $\omega+10$ to the C-terminus (Pierleoni et al., 2008) (Figure 12). Thus, SMAC_12074 appeared to encode a pre-protein posttranslationally cleaved at the $\mathrm{N}$-terminal and C-terminal regions and modified by attachment of a C-terminal GPI-anchor. 


\begin{tabular}{|c|c|c|}
\hline $\begin{array}{l}\text { Signal } \\
\text { sequence }\end{array}$ & & $\begin{array}{r}\text { Region } f \\
\text { I-anchor att }\end{array}$ \\
\hline aa $1-27$ & SmGPI1 & aa $216-253$ \\
\hline
\end{tabular}

Figure 12. Schematic illustration of the SmGPI1 precursor. N-terminally located grey colored signal sequence was predicted by SignalP (Bendtsen et al., 2004, Emanuelsson et al., 2007). C-terminally grey colored region for GPI-anchor attachment was predicted by big-PI-Predictor (Eisenhaber et al., 1998, Eisenhaber et al., 1999, Eisenhaber et al., 2000, Sunyaev et al., 1999). Putative processing sides are indicated by arrows. The black boxed omega residue is predicted to be aa 228. This aa is exposed for GPI-anchor attachment by a GPI transamidase (Mayor \& Riezman, 2004).

Based on these findings, SMAC_12074 was named Smgpi1 to indicate that it encodes the first described GPI-anchored protein in S. macrospora. Sequence alignment of SmGPI1 with 7 putative homologs from other fungi revealed conservation of this protein among filamentous ascomycetes (Figure 13A).

All identified proteins were predicted to have a signal sequence and a region for GPI-anchor attachment. Based on aa sequences, N. crassa NCU09375 shared the highest level of identity to SmGPI1 (91\%), followed by Podospora anserina with (49\%), Magnaporthe oryzae and Chaetomium globosum (45\%) (Figure 13B). 

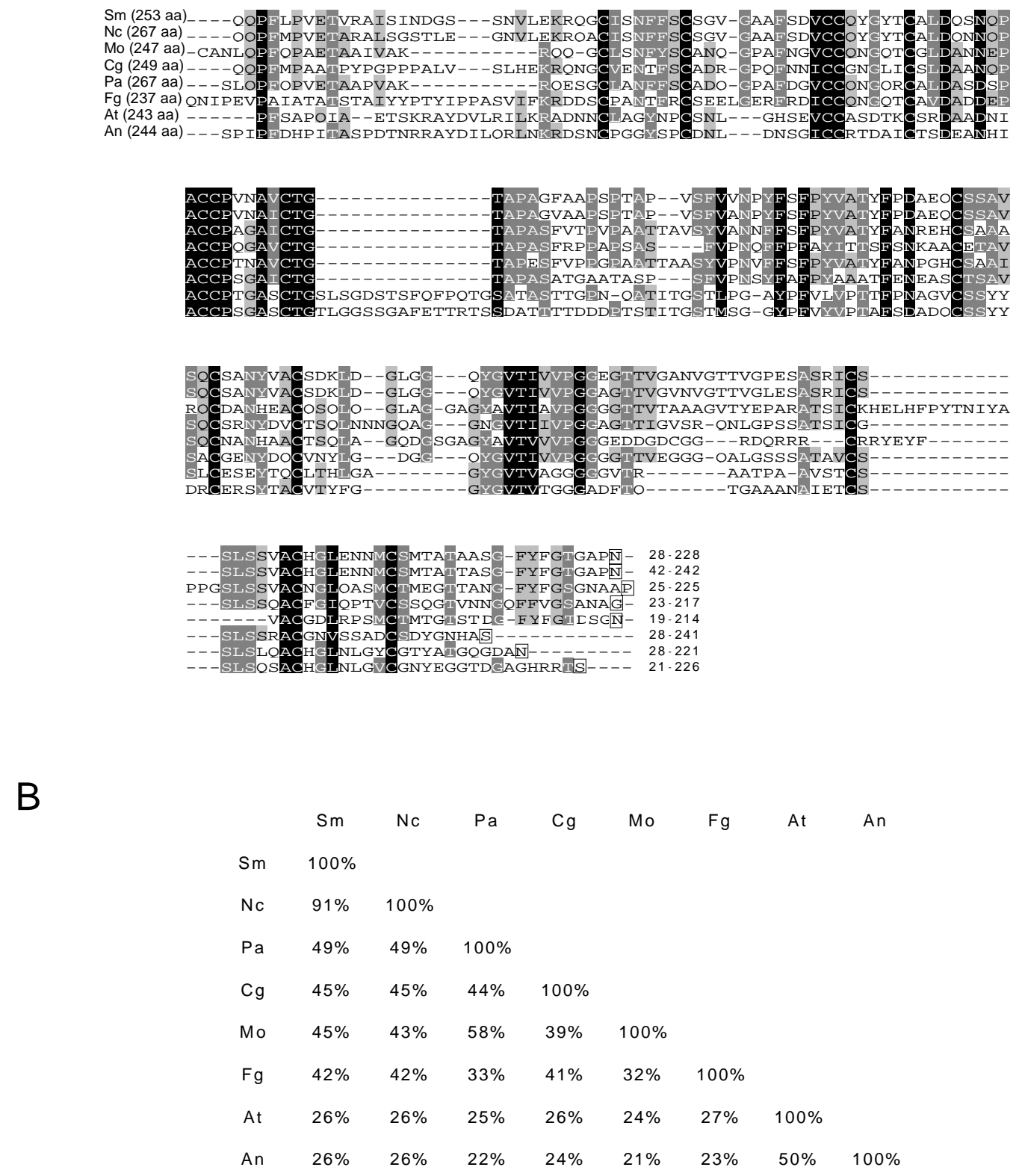

Figure 13. Multiple sequence alignment and aa identity of SmGPI1 with putatively homologue proteins from other Ascomycota. (A) Alignment was made using ClustalX2 (Larkin et al., 2007). Sm, Sordaria macrospora, F7W197; Nc, Neurospora crassa, Q7SCN6; Mo, Magnaporthe oryzae, G4MNK5; Cg, Chaetomium globosum, Q2HAL9; Pa, Podospora anserina, B2AM33; Fg, Fusarium graminearum I1RCP4; At, Aspergillus terreus, Q0CT61; An, Aspergillus nidulans, Q5B70. Total numbers of aa are given in brackets. For alignment only aa sequences downstream of the putative signal sequence up to the omega site were used. (B) Identity of the aligned aa sequences in pair-wise comparison. 


\subsubsection{SmGPI1 interacts physically with SmMOB3}

Using the Y2H system we confirmed that SmGPI1 physically interacted with SmMOB3 in S. cerevisiae (Figure 14). Full-length and truncated Smgpi1 cDNAs were cloned into the GAL4 DNA-binding domain of the Y2H vector pGBKT7. Plasmid pAD-SmMOB3 encoding Smmob3 full-length protein, and its derivative pAD-SmMOB3 aa 1-144 encoding for the N-terminally truncated version, were used as prey vectors. Plasmids were transformed into yeast strains MATa Y187 (pGBKT7 constructs) or MAT $\alpha$ AH109 (pGADT7 constructs). The pGBKT7-SmGPI1 bait-constructs were checked for transactivation activity by mating Y187 transformants with yeast strain AH109 carrying the empty pGADT7 plasmid (data not shown). A strain carrying both empty plasmids served as negative control. Interaction of PRO11 and SmMOB3 served as positive control (Bloemendal et al., 2012). Expression of GAL4 fusion proteins from pGBKT7SmGPI1 plasmids was also checked by mating Y187 transformants with AH109 carrying pADRanBPM (Tucker et al., 2009) (data not shown).

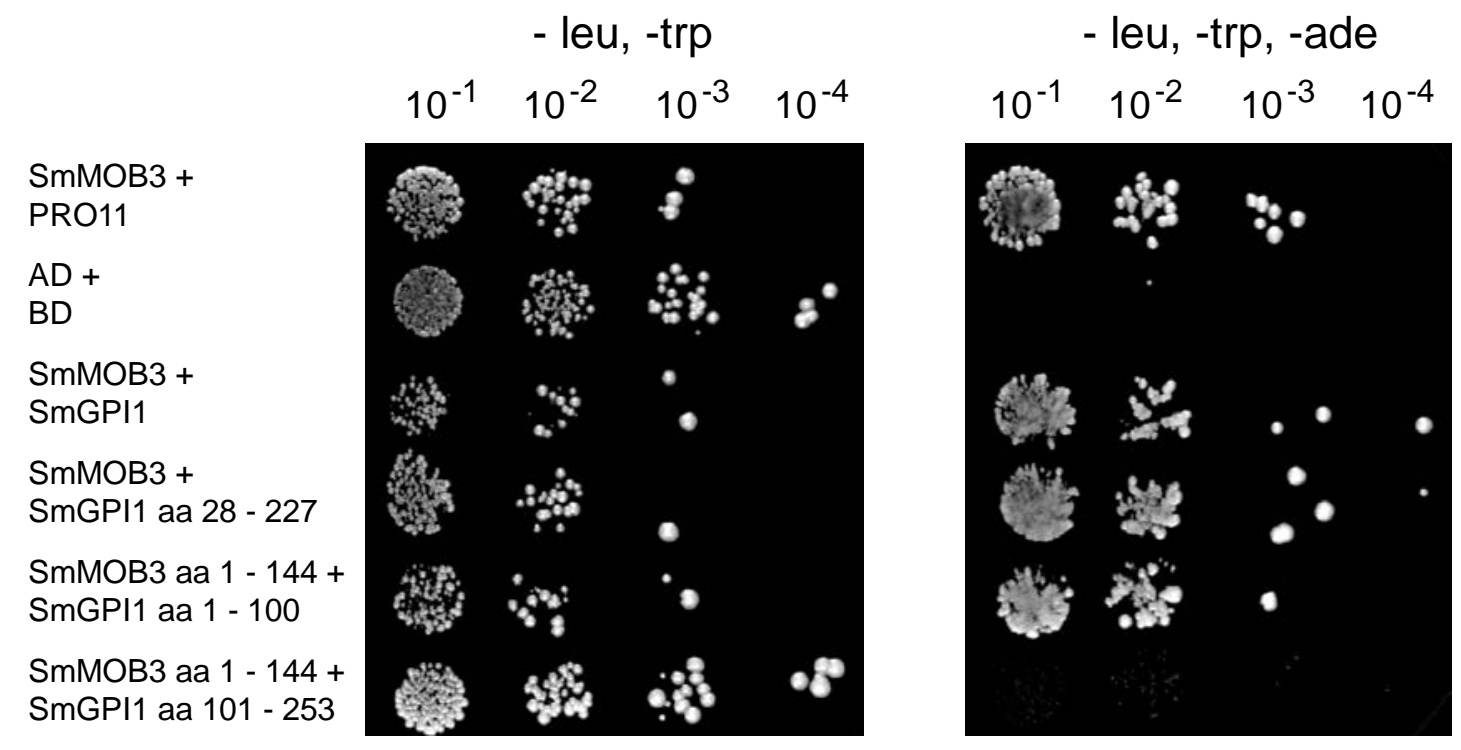

Figure 14. Yeast two-hybrid analysis of the interaction of SmGPI1 and SmMOB3. Serial dilutions of diploid yeast strains obtained after mating spread on SD medium lacking tryptophan (trp) and leucine (leu) or trp, leu and adenine (ade) to verify the interaction of both proteins. Full-length and truncated versions of SmGPI1 and SmMOB3 were tested. BD = DNA-binding domain of GAL4, AD = activation domain of GAL4. GAL4 binding domain was fused to SmGPI1, GAL4 activating domain to SmMOB3. Reverse application of activation and binding domain was not possible due to transactivation of SmMOB3. Yeast transformants carrying pAD-SmMOB3 (SmMOB3) and pBD11 aa 282-845 (PRO11) served as positive control (Bernhards and Pöggeler, 2011). As negative control a diploid strain carrying empty vectors pGADT7 (AD) and pGBKT7 (BD) was used. 
Y2H results demonstrated interaction between SmGPI1 and SmMOB3 (Figure 14). Neither the signal sequence nor the region for GPI-anchor attachment was necessary for SmGPI1/SmMOB3 interaction. The N-terminal regions of SmGPI1 (aa 28-100) and SmMOB3 (aa 1-144) mediated interaction (Figure 14). To verify physical interaction of SmMOB3 and SmGPI1 in vivo, we performed co-Immunoprecipitation (co-IP) studies in S. macrospora. We expressed functional, N-terminally tagged FLAG-SmMOB3 (Bloemendal et al., 2012) and an eGFP-tagged full-length SmGPI1 in S. macrospora. As SmGPI1 was predicted to be posttranslationally cleaved at the Nterminus and C-terminus, we fused eGFP upstream of the linker of the GPI attachment region between aa position 217 and 218 (SmGPI1-eGFP aa 1-492) (Figure 12). Tagged versions of the proteins were separately expressed and co-expressed in S. macrospora wt transformants. Co-IP confirmed the physical interaction of SmGPI1-eGFP aa 1-492 and full length FLAG-SmMOB3 (Figure 15).
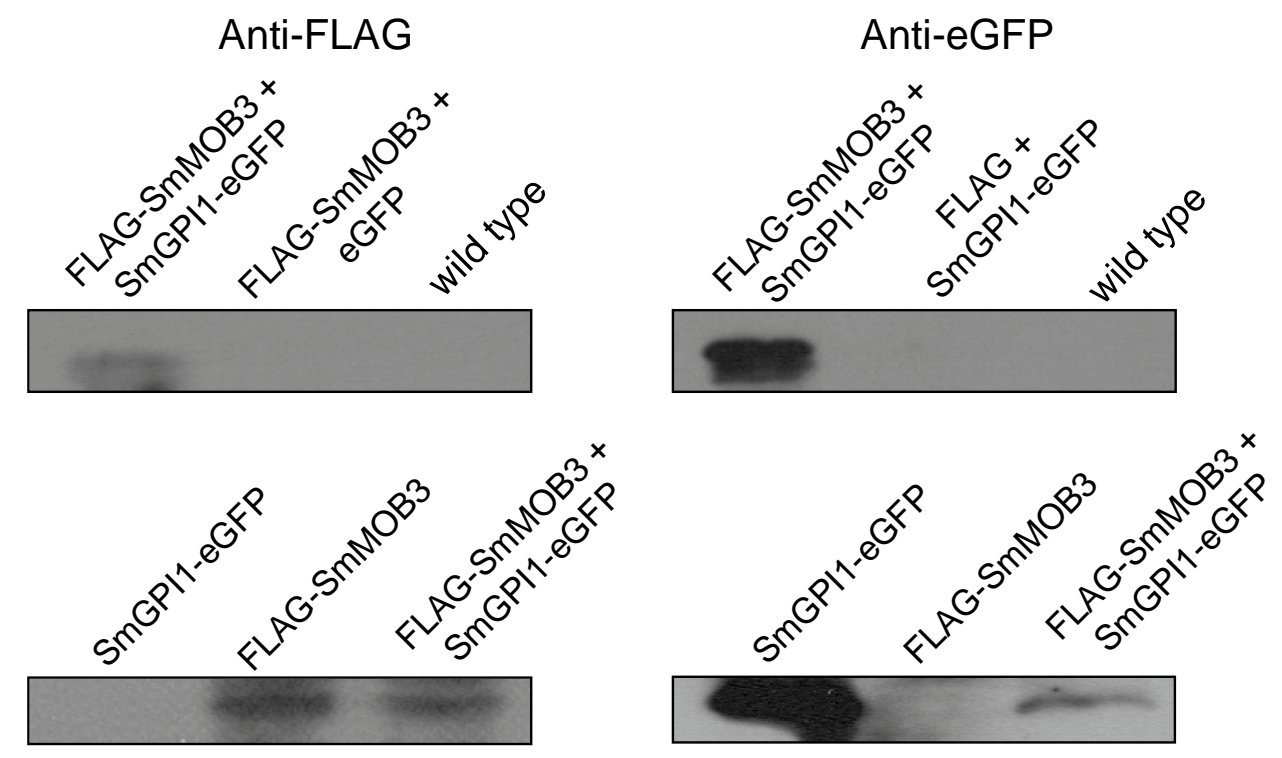

Figure 15. Co-Immunoprecipitation of SmGPI1 and SmMOB3 with anti-FLAG and anti-eGFP antibodies combined with Western blot analysis. Separately expressed constructs and SmGPI1 co-expressed with FLAG or SmMOB3 co-expressed with eGFP served as control. Shown are SmMOB3-FLAG, fished with SmGPI1-eGFP and vice versa. 


\subsubsection{SmGPI1 binds to the cell wall and is partially secreted}

SmGPI1 was predicted to have a signal sequence and a region for GPI-anchor attachment (Figure 12). To verify this prediction, plasmids encoding SmGPI1-eGFP aa 1-492 and SmGPI1-eGFP aa 1-466, lacking the GPI-anchor attachment region (Figure 16A) were transformed into a S. macrospora wt strain. Crude extracts were fractionated by differential centrifugation and analyzed by Western blot (Figure 16B).

A

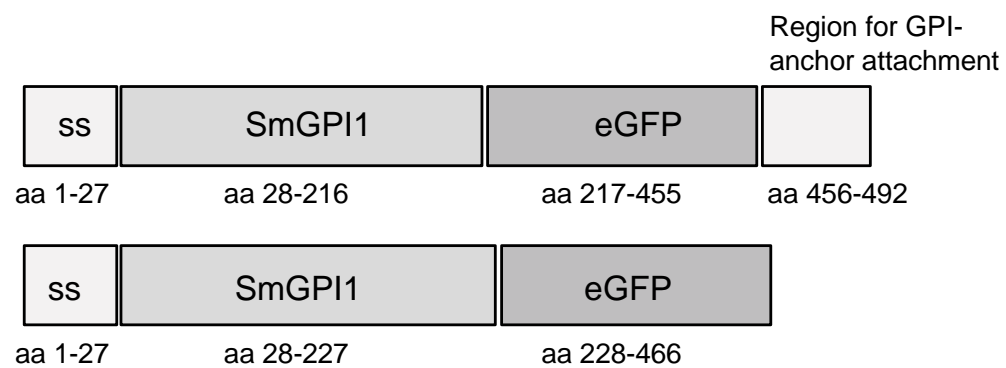

B

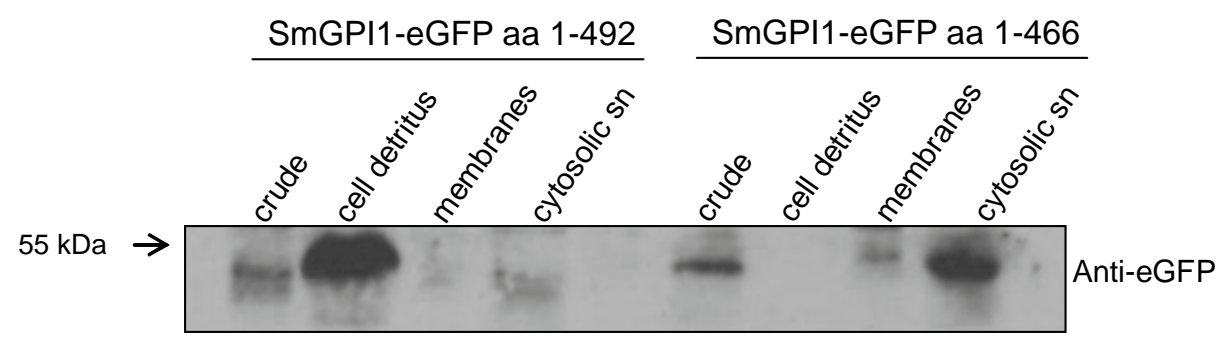

Figure 16. Western blot analysis of SmGPI1 after differential centrifugation of cellular components. (A) Schematic overview of SmGPI1 versions used in this analysis. SmGPI1-eGFP aa 1-492 consists of SmGPI1 aa 1-216 fused to eGFP followed by the region for GPI-anchor attachment $\omega-10$ to $\omega+25$ of SmGPI1. The $\omega$-residue is predicted to be aa 228 when eGFP is introduced between position 217 and 218 it changes to aa position 466. SmGPI1-eGFP aa 1-466 lacks the C-terminal region for GPI-anchor attachment $(\omega-\omega+25)$. (B) Western blot using eGFP antibody. SmGPI1-eGFP aa 1-492 is predominantly found in the cell detritus containing remnants of the cell wall. SmGPI1 aa 1-466 is mainly present in the cytosolic supernatant (sn) after ultracentrifugation. Samples were separated on 15\% SDS-PAGE, blotted on nitrocellulose membranes and probed with anti-eGFP antibody.

SmGPI1-eGFP aa 1-492 appeared predominantly in the fractions containing remnants of the cell wall (cell detritus), whereas SmGPI1-eGFP aa 1-466 was mainly present in the cytosolic 
supernatant but not in the fraction containing the cell detritus (Figure 16B). Thus, SmGPI1 seemed to be mainly localized to the cell wall.

Three further SmGPI1-eGFP versions were used to analyze the functionality of the predicted signal sequence (Figure 17A). SmGPI1-eGFP aa 28-492 lacked the signal sequence for secretion. The signal sequence of SmGPI1 was fused to eGFP in SmGPI1-eGFP aa 1-27. The version SmGPI1-eGFP aa 1-27-KDEL carried the C-terminal ER retention signal Lys-Asp-Glu-Leu (Pelham, 1990).

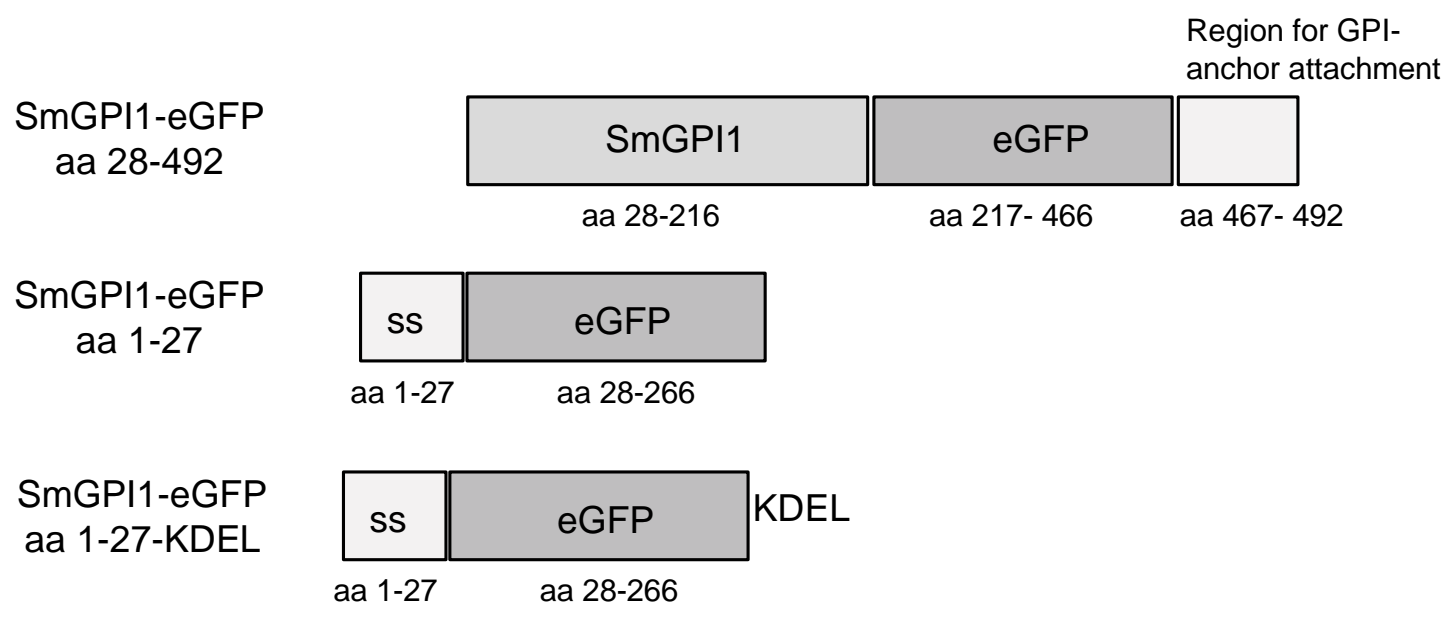

Figure 17. Schematic overview of SmGPI1 versions used for Western blot (see also Figure 16). SmGPI1eGFP aa 28-492 consists of SmGPI1 aa 28-216 fused to eGFP followed by the region for GPI-anchor attachment $\omega-10$ to $\omega+25$ of SmGPI1. SmGPI1-eGFP aa 1-27 consists of aa 1-27 of SmGPI1 fused to eGFP. SmGPI1-eGFP aa 1-27-KDEL carries additionally the ER retention signal KDEL.

Protein extracts from mycelium ground in liquid nitrogen and cell-free supernatants from strains expressing genes encoding SmGPI1-eGFP aa 1-492, SmGPI1-eGFP aa 28-492 and SmGPI1eGFP aa 1-466 were analyzed by Western blot (Figure 18B). SmGPI1-eGFP aa 28-492 was seen only in the mycelium sample, whereas SmGPI1-eGFP aa 1-492 and SmGPI1-eGFP aa 1-466 were also detected in cell-free supernatant. Two signals were observed in the liquid medium of transformants expressing full-length SmGPI1-eGFP aa 1-492. The faster migrating signal was the size of free eGFP ( $27 \mathrm{kDa})$ and was probably a degradation product of the full-length SmGPI1-eGFP aa 1-492 fusion protein that appeared as a protein of $52 \mathrm{kDa}$ (Figure 18B). To ensure cell-free supernatants we used anti-actin antibody as control. 

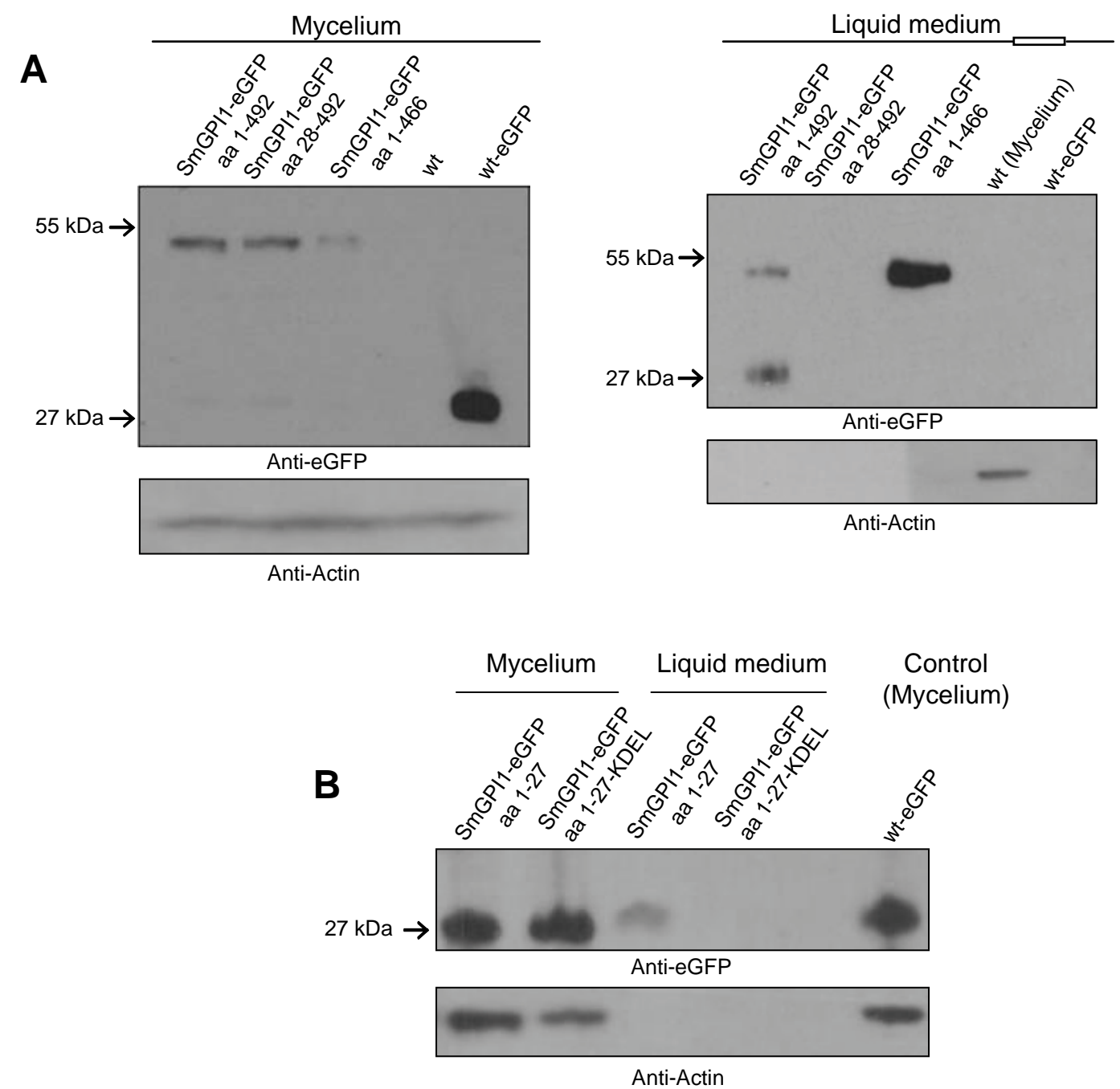

Figure 18. Western blot analysis of SmGPI1 aa 1-492 and aa 28-492 using cell-free supernatants and crude extracts of the mycelium. (A) Total cellular (mycelium) and total secreted (liquid medium) proteins were separated by SDS-PAGE and visualized by Western blotting using an anti-eGFP antibody. For the Western blot of the mycelial crude extracts the protein extract of the wt was used as a negative control and wt expressing egfp as a positive control. Actin served as loading control and was visualized anti-actin antibody was used. In addition, anti-actin antibody was used to verify that no cellular proteins are present in the liquid medium. Total protein extract of the wt served as a positive control for anti-Actin antibody. (B) Western blot analysis of the functionality of SmGPI1 signal sequence. SmGPI1 aa 1-27 was fused to eGFP optionally tagged with ER-retention signal KDEL and visualized by anti-eGFP antibody. Actin was used as a loading control and visualized by an anti-Actin antibody.

To further investigate the functionality of the predicted signal sequence, the coding sequence of the first 27 aa of SmGPI1 was fused to egfp (SmGPI1-eGFP aa 1-27). As a control, the construct was C-terminally tagged with the ER retention signal KDEL (SmGPI1-eGFP aa 1-27-KDEL). Western blot showed that only eGFP N-terminally tagged with the signal sequence and lacking 
the ER retention signal was detected in cell-free supernatants. After tagging this construct with an ER retention signal it no longer appeared in the cell-free supernatant (Figure 18C). Thus, the signal sequence of SmGPI1 was sufficient to mediate eGFP secretion.

\subsubsection{SmGPI1 localizes to the cell wall and mitochondria}

Fluorescence microscopy was performed to determine the localization of SmGPI1 in vivo. Strains expressing genes coding for SmGPI1-eGFP aa 1-492, SmGPI1-eGFP aa 28-492, SmGPI1-eGFP aa 1-466 and SmGPI1-eGFP aa 28-466 were analyzed (Figure 16 and Figure 17). SmGPI1-eGFP aa 1-492 was detected at the cell wall and intracellular structures resembling mitochondria. For verification, hyphae were co-stained with the membrane dye FM 4-64 or MitoTracker Red (Figure 19). SmGPI1-eGFP aa 1-492 co-localized with the membrane dye FM 4-64 and MitoTracker Red. MitoTracker Red co-staining was displayed by focusing into the intracellular lumen. In contrast to the localization of the full-length protein, the N-terminally truncated SmGPI1-eGFP aa 28-492 and SmGPI1-eGFP aa 28-466 were diffusely distributed within the cytosol and did not co-localize with FM 4-64 or MitoTracker Red. However, SmGPI1-eGFP aa 1-466, containing the putative N-terminal secretion signal, localized to mitochondria but not to the cell wall.

Localization of SmGPI-eGFP did not change in $\Delta$ Smmob3. The protein localized to the plasma membrane and mitochondria. Similarly, the distribution of SmMOB3-eGFP to the nuclear envelope and cytoplasm did not change in $\Delta$ Smgpil, as shown in Figure 20. 


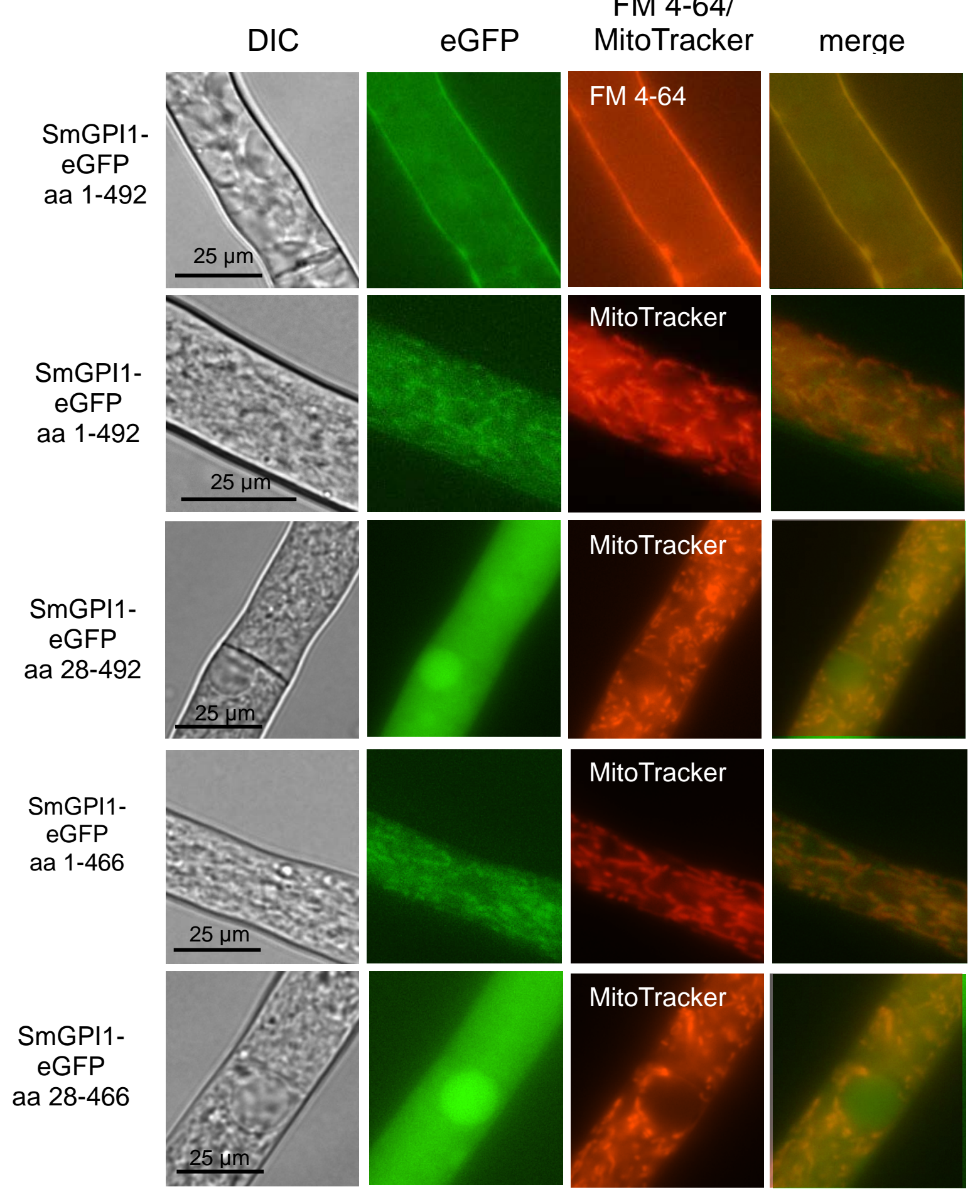

Figure 19. Localization of SmGPI1-eGFP. Deletion of the omega side aborts localization to the cell wall but maintained localization to mitochondria. Deletion of the signal sequence results in cytosolic localization. For visualization was focused either to the cell wall or to the cytoplasm. 
A

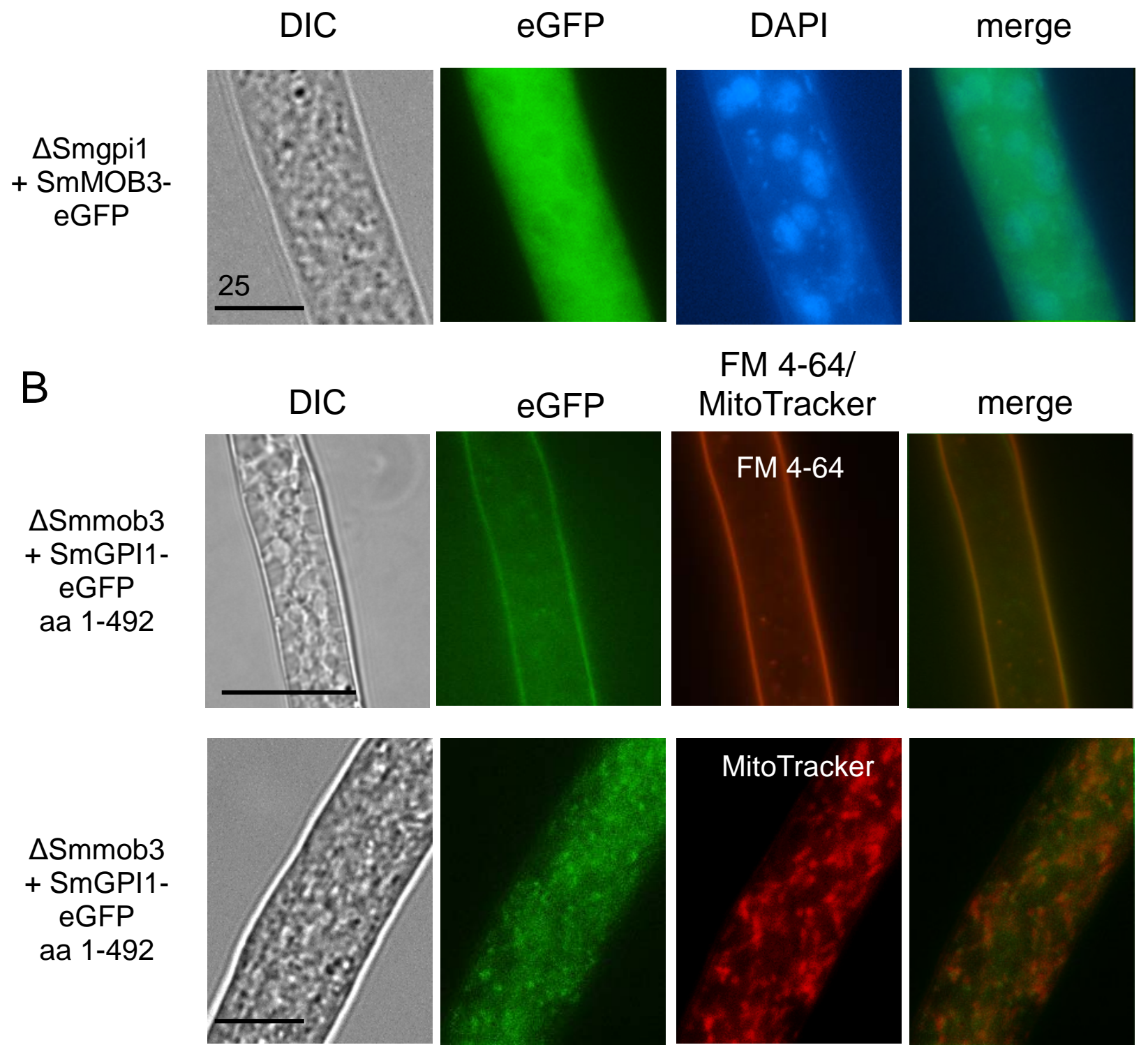

Figure 20. Localization of SmMOB3 in $\triangle$ Smgpil and SmGPI1 in $\triangle$ Smmob3. (A) SmMOB3 localizes to the nuclear envelope in $\triangle$ Smgpi1. (B) SmGPI1 localizes to the cell membrane and mitochondria in $\Delta$ Smmob3. For visualization of cell membranes cells were stained with FM 4-64, mitochondria were stained with MitoTracker Red as described in material and methods. DAPI was used for nuclei staining. Scale bar as indicated. 


\subsubsection{Deletion of Smgpi1 restores fertility and hyphal fusion of sterile $\Delta$ Smmob3}

Previous studies of the S. macrospora STRIPAK complex including SmMOB3 revealed that it is intracellularly localized (Bernhards \& Pöggeler, 2011, Bloemendal et al., 2012). To analyze the interplay of the intracellular protein SmMOB3 and the GPI-anchored protein SmGPI1 in more detail, a single $\Delta$ Smgpil mutant and a double $\Delta$ Smgpi $1 / \Delta$ Smmob3 mutant were generated. Smgpi1 was replaced with a hygromycin-resistance cassette via homologous recombination in a $\Delta$ ku70 strain (Pöggeler \& Kück, 2006) (Figure 21).

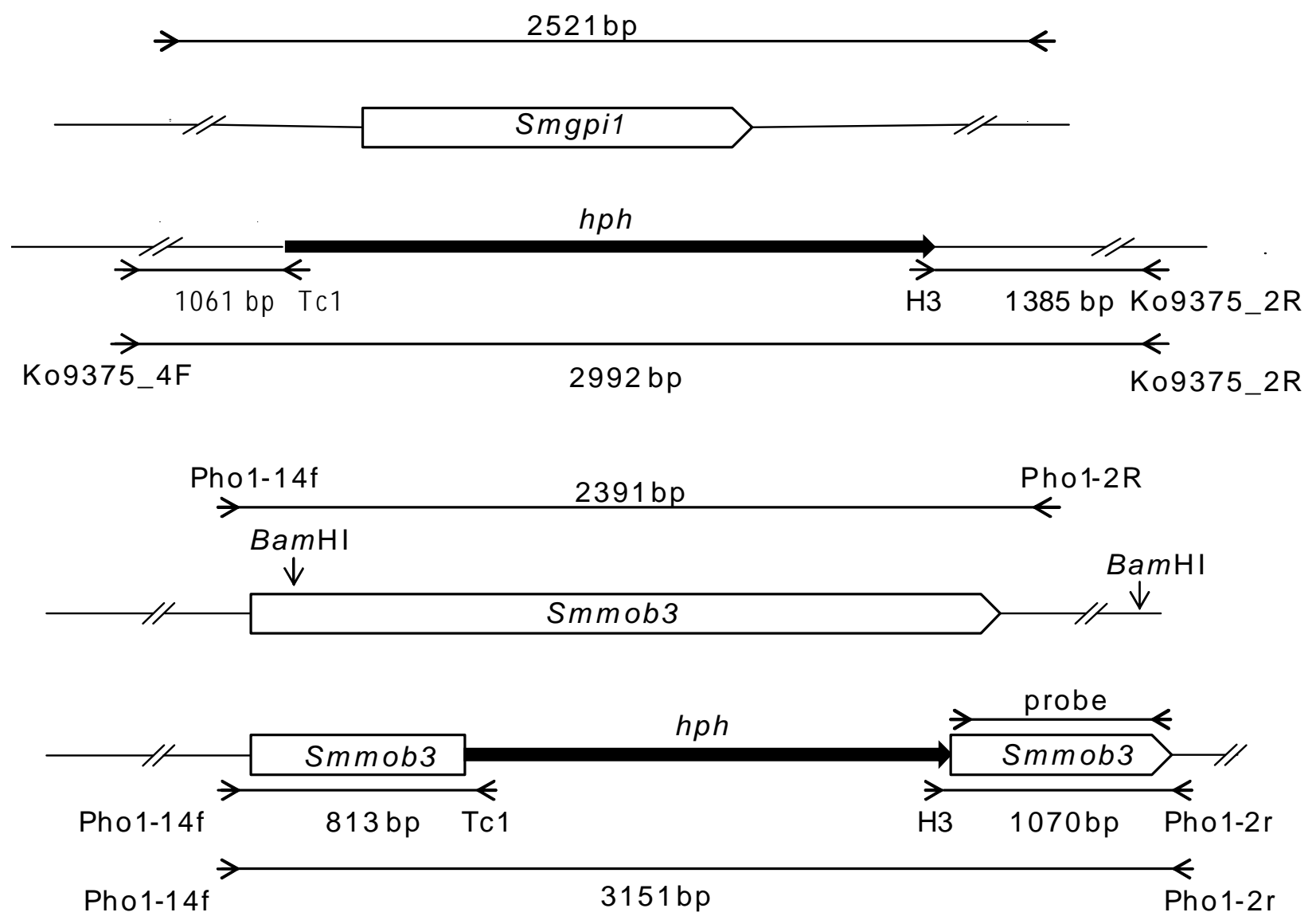

Figure 21. Generation of a $\Delta$ Smgpil/ $\Delta$ Smmob3 double-deletion strain. Schematic illustration of the Smgpi1 and Smmob3 locus before and after homologous integration of the deletion cassette. Primers used for the verification of the respective gene deletion are shown by arrows. Sizes of PCR fragments as well as for the probe used for Smmob3 Southern hybridization are given.

The homokaryotic $\Delta \mathrm{ku} 70 / \Delta$ Smgpil mutant was crossed to the spore-color mutant fus1-1 (Nowrousian et al., 2012) to eliminate the ku70 deletion background. Gene deletion was verified 
by PCR and Southern blot hybridization (Figure 22). Subsequently, $\Delta$ Smgpil was phenotypically analyzed with regard to sexual development. $\Delta$ Smgpi1 was fertile and completed the life cycle within 7 days (Figure 23). Similar to wt, first ascogonia were visible after 3 days, developing to non-pigmented protoperithecia at day 4 and pigmented protoperithecia at day 5. Two days later, mature fruiting bodies containing ascospores were formed.

A

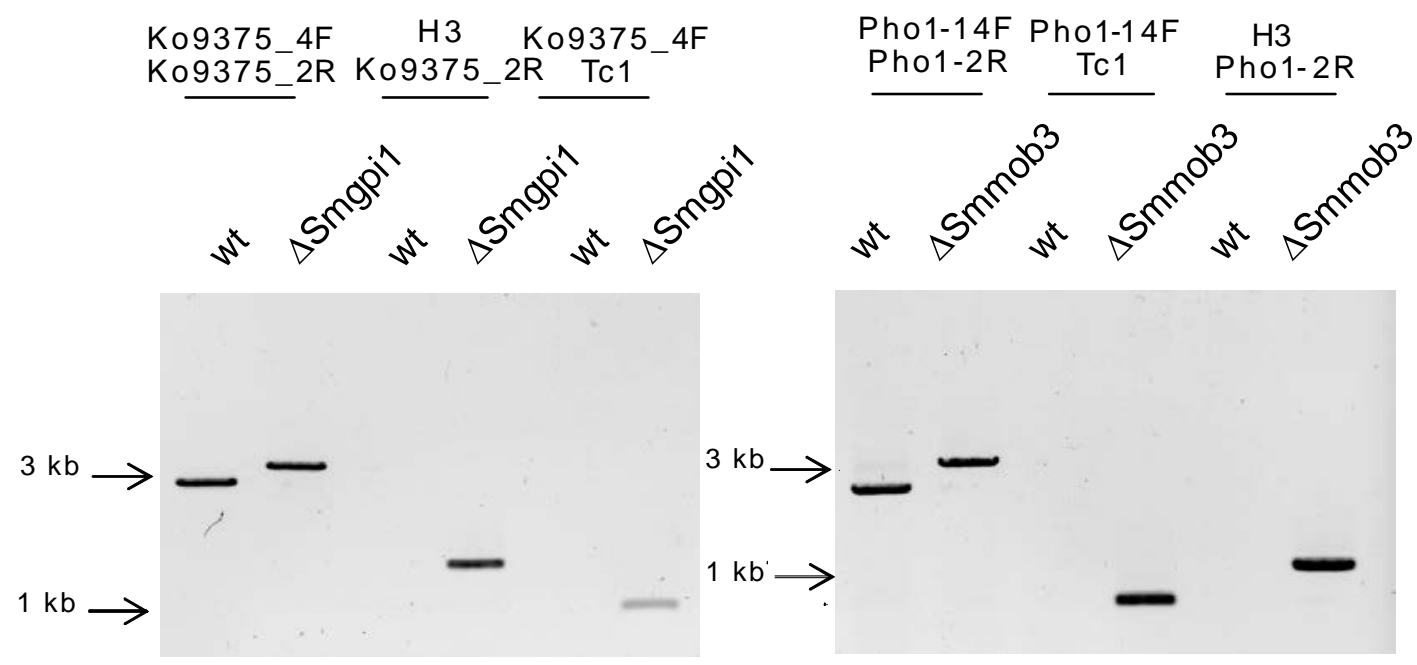

B
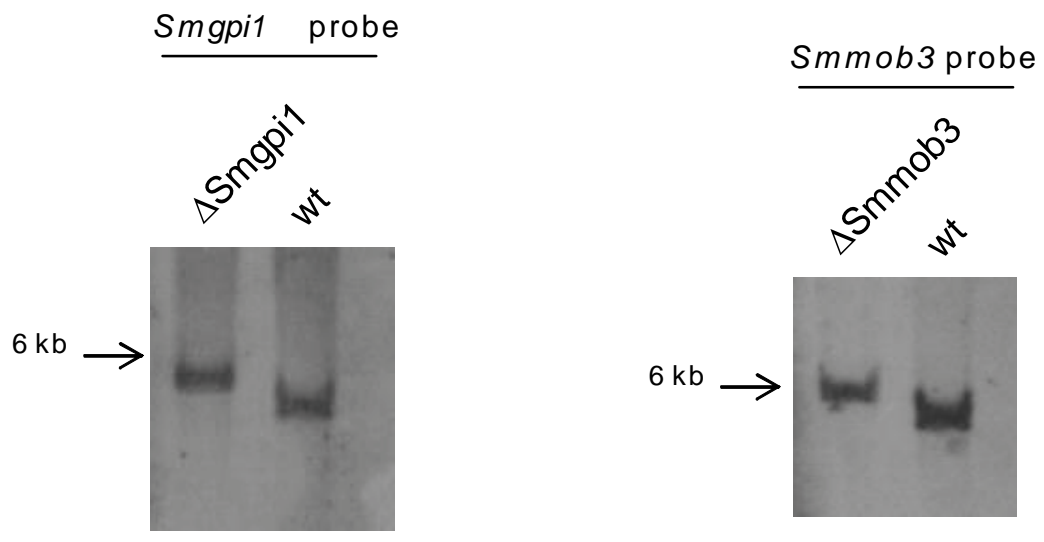

Figure 22. Verification of $\Delta$ Smgpi $1 / \Delta$ Smmob3 via PCR and Southern blot. (A) Verification of the deletion using PCR. Shown are the calculated fragment sizes for wt and the gene deletions. (B) Southern hybridization (Sambrook \& Russell, 2001) for Smmob3 confirmed the successful integration of the deletion cassette via gene-specific probe.

In other organisms, genes coding for GPI-anchored proteins are sensitive to cell membrane and cell wall stress inducing agents (Sestak et al., 2004, Vaknin et al., 2014). To test stress 
conditions on Smgpi1, we performed growth tests on supplemented solid media. The $\Delta$ Smgpi1 strain showed no sensitivity to cell wall stress agents calcofluor white and caffeine or to the cell wall degrading enzymes polygalacturonase or arabanase as present in Natuzym (data not shown). In addition, $\Delta$ Smgpil was as sensitive as wt to different types of stresses induced by $\mathrm{NaCl}, \mathrm{KCl}$, sorbitol, menadione, SDS, $\mathrm{H}_{2} \mathrm{O}_{2}$ or low and high $\mathrm{pH}$ (data not shown). Growth velocity was tested in race tubes with fructification medium over 10 days. The average growth of $\Delta$ Smgpil was $29 \pm 4 \mathrm{~mm} /$ day which is similar to wt growth (29 $\pm 5 \mathrm{~mm} /$ day) (Table 5$)$.

Table 5. Average growth value of $\Delta$ Smgpi1, complemented $\Delta$ Smgpil (Smgpi1+), $\Delta$ Smmob3 and the double knockout $\Delta$ Smgpi1/ $\Delta$ Smmob3 in mm/day, measured over 10 days on SWG medium.

Strain

wild type

$\Delta$ Smgpil

$\Delta$ Smgpi1+

$\Delta$ Smmob3

$\Delta$ Smgpi $1 / \Delta$ Smmob3

\section{Growth velocity (mm/day)}

$$
\begin{gathered}
29,5 \pm 5 \\
29,4 \pm 4 \\
30,7 \pm 6 \\
8 \pm 3 \\
25,5 \pm 5
\end{gathered}
$$

The double deletion strain $\Delta$ Smgpil/ $\Delta$ Smmob3 was constructed by crossing the single-deletion strains $\Delta$ Smgpil and $\Delta$ Smmob3 (Bernhards \& Pöggeler, 2011) (Figure 23). In S. macrospora and $N$. crassa, MOB3 is required for hyphal fusion and fruiting-body development (Bernhards \& Pöggeler, 2011, Fu et al., 2011, Maerz et al., 2009). Deletion of Smgpi1 in the sterile $\Delta$ Smmob3 deletion background restored fertility (Figure 23). In contrast to the single-deletion strain $\Delta$ Smmob3, $\Delta$ Smgpi1/ $\Delta$ Smmob3 completed the life cycle but after a prolonged time of 13 days. Thus, the double-deletion mutant was fertile, but had delayed sexual development (Figure 23). Transformation of $\Delta$ Smgpi1/ $\Delta$ Smmob3 with the Smgpi1 wt gene resulted in sterility (Figure 24); therefore, fertility of the double-deletion mutant was caused by deletion of Smgpi1 in the sterile $\Delta$ Smmob3 background. 


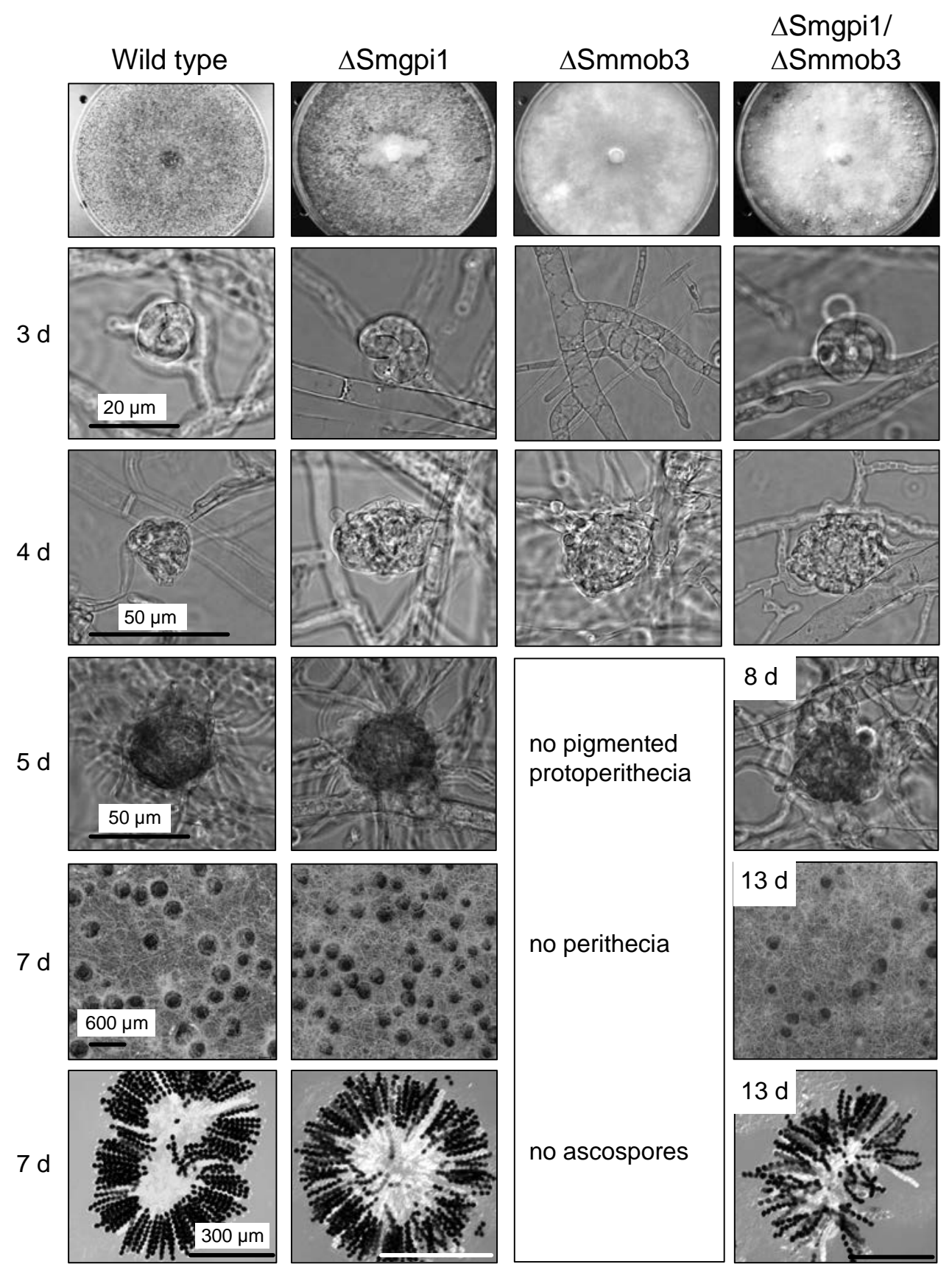

Figure 23. Sexual development of $\Delta$ Smgpi1, $\Delta$ Smmob3 and $\Delta$ Smgpi1 $/ \Delta$ Smmob3. Shown are the respective deletion strains compared to wt. The wt strain generates ascogonia after 3 days, which develop to protoperithecia at day 5 and within 7 days to mature perithecia containing the ascospores. $\Delta$ Smgpi 1 completes the life cycle within 7 days, whereas $\Delta$ Smmob3 develops only protoperithecia. Fruiting-body development in the $\Delta$ Smgpi1/ $\Delta$ Smmob3 mutant is delayed. Strains were inoculated on solid SWG medium. 
To ensure that the observed genetic interaction between Smmob3 and Smgpi1 is specific, additional double-deletion mutants lacking genes encoding other STRIPAK components such as $\Delta$ pro11 (Bernhards \& Pöggeler, 2011, Bloemendal et al., 2012), $\Delta$ pro22 (Bloemendal et al., 2012) or $\Delta$ pro45 (Nordzieke et al., 2014) and $\Delta$ Smgpi1 (Figure 25, Figure 26 and Figure 27) were generated by crosses.

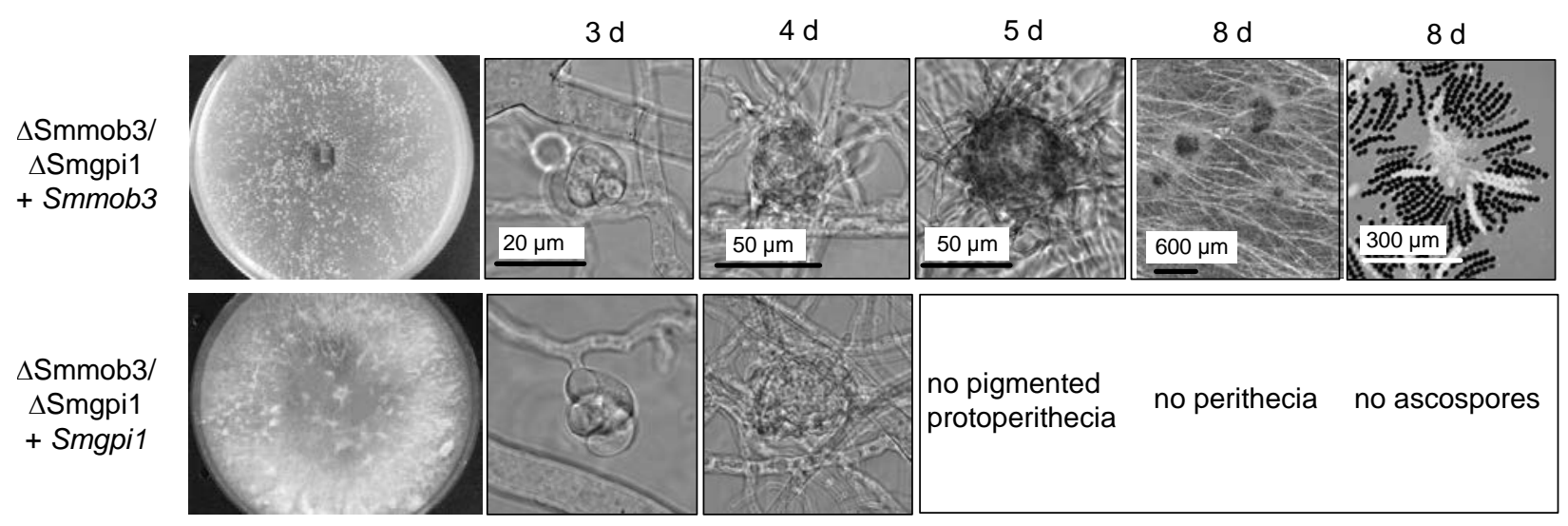

Figure 24. Phenotypic analysis of $\Delta$ Smgpi $/ \Delta$ Smmob3 complemented with full-length Smgpi1 or Smmob3 using microscopy. $\Delta$ Smgpi $1 / \Delta$ Smmob3 + Smmob3 completes the life cycle within 8 days whereas $\Delta$ Smgpi1/ $\Delta$ Smmob3 + Smgpi1 only develops protoperithecia. Smgpi1 and Smmob3 were under control of their native promotor and terminator. Both complemented double-deletion strains are phenotypically identical to the respective single-deletion strain.

Similar to deletion of Smmob3, mutation or deletion of pro11 or pro22 led to sterility of $S$. macrospora (Bernhards \& Pöggeler, 2011, Bloemendal et al., 2012, Bloemendal et al., 2010, Pöggeler \& Kück, 2004). The double-deletion mutants $\Delta$ Smgpil/spro11 (Figure 25), $\Delta$ Smgpi1/ $\Delta$ pro22 (Figure 26) or $\Delta$ Smgpil/ $\Delta$ pro45 (Figure 27) did not reverse the sterile phenotype as $\Delta$ Smgpi1/ $\Delta$ Smmob3. Furthermore, a double-deletion $\Delta$ SMAC_03978/ $\Delta$ Smmob3 strain, lacking Smmob3 and SMAC_03978, an unrelated gene encoding a hypothetical GPIanchored protein, was generated. Deletion of the unrelated gene did not suppress the sterile phenotype of $\Delta$ Smmob3 (Figure 28). Thus, suppression of $\Delta$ Smmob3 by deletion of Smgpi1 is a specific effect and suggested genetic interaction. 

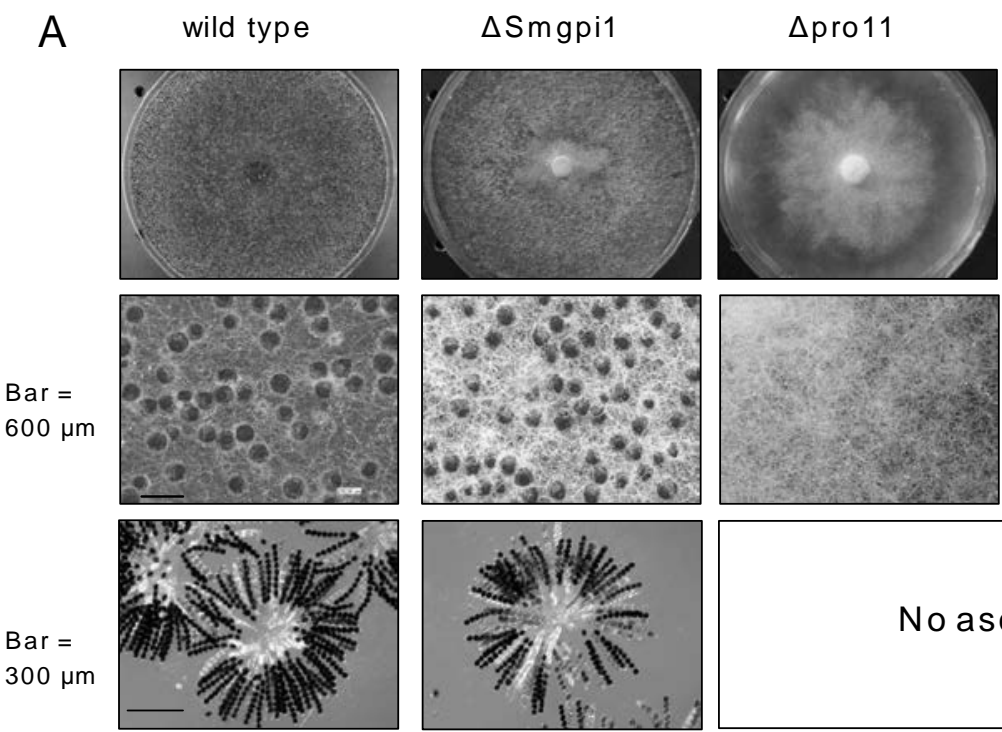

$\Delta$ Smgpi1/Dpro11
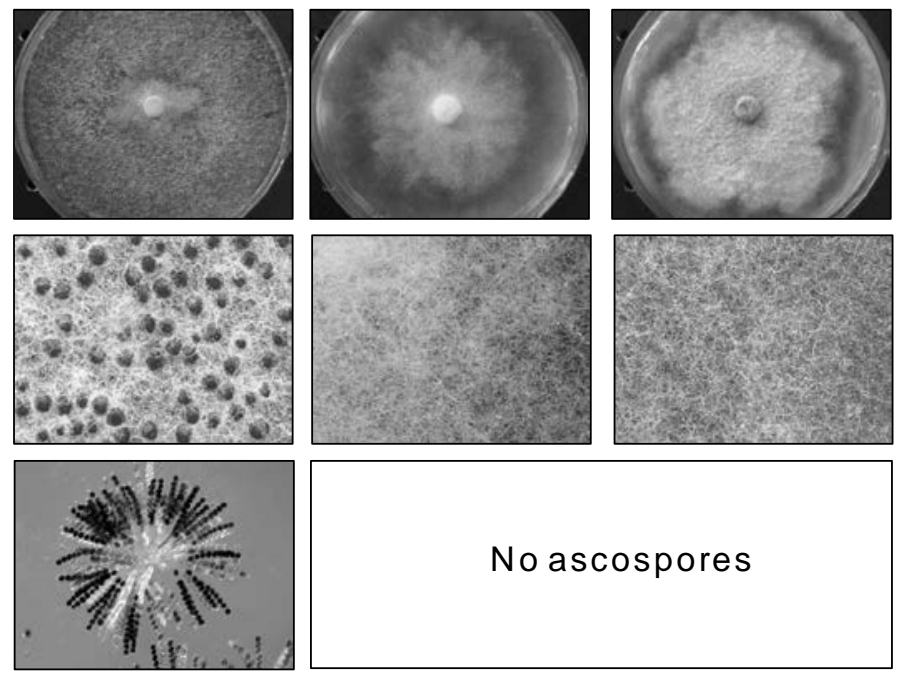

B

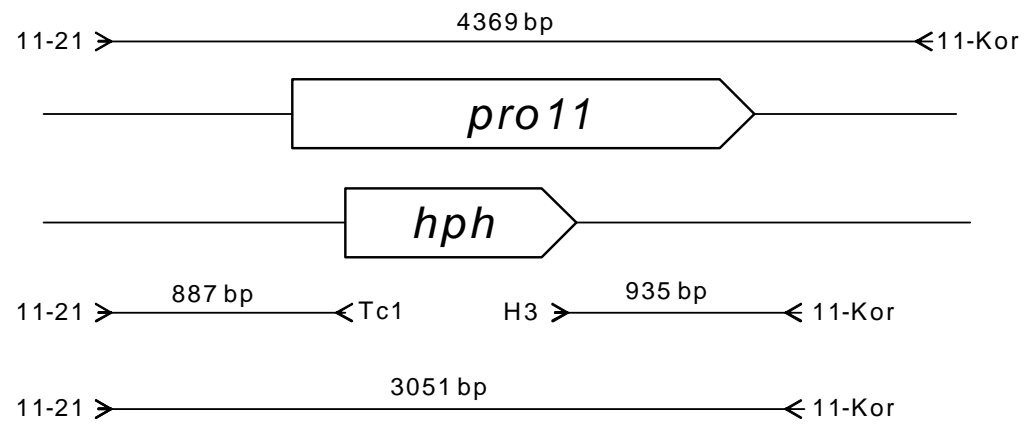

C

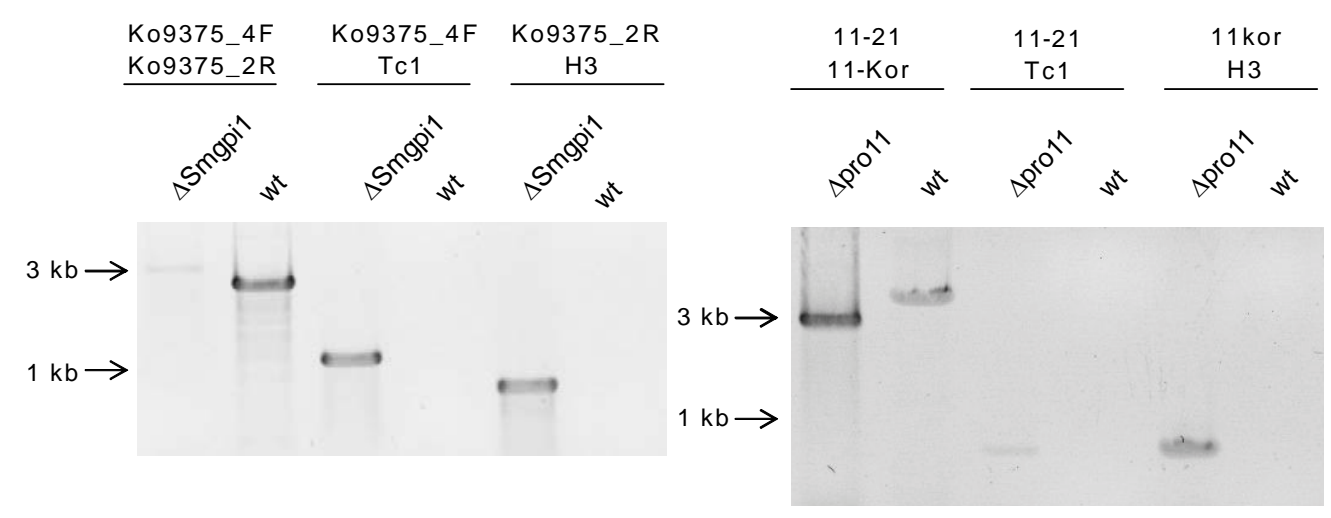

Figure 25. Generation of a $\Delta$ Smgpi1/ $\Delta$ pro11 double-deletion strain. (A) Phenotype of $\Delta$ Smgpi1/ $\Delta$ pro11, respective single-knockout strains and wt with focus on fruiting-body development. (B) Schematic illustration of the pro11 locus (Pöggeler and Kück, 2004) before and after homologous integration of the deletion cassette. Primers used for verification of the deletion strain are shown by arrows. PCR-fragment sizes are given. (C) Verification of gene deletions in the $\Delta$ Smgpil/Apro11 using PCR. Shown are the calculated fragment sizes for wt and the respective gene deletion. Strains were obtained by crossing single-deletion strains $\Delta$ Smgpil/r2 and $\Delta$ pro11. 

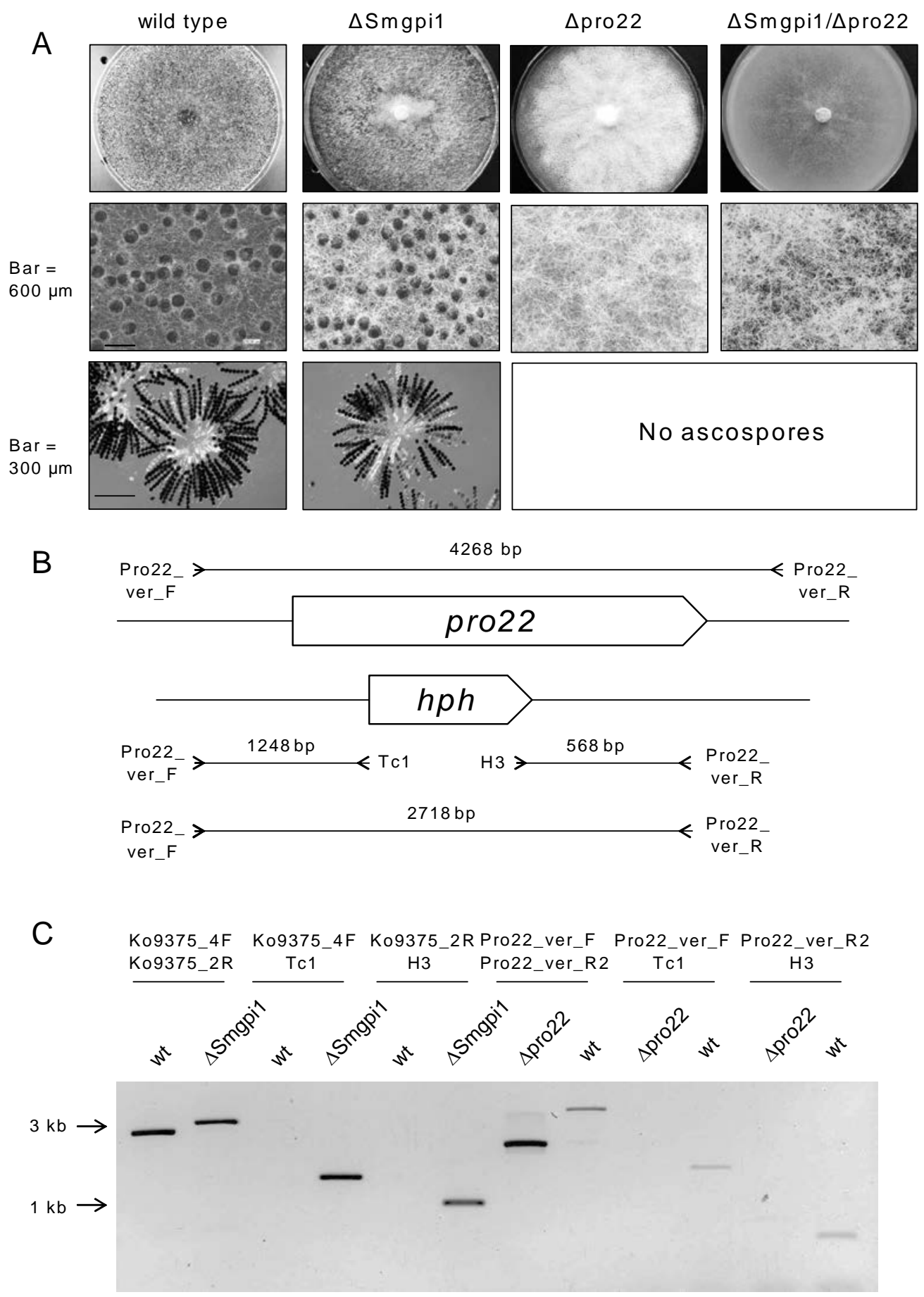

Figure 26. Generation of a $\Delta$ Smgpi1/ $\Delta$ pro22 double-deletion strain. (A) Phenotype of $\Delta$ Smgpi1/ $\Delta$ pro22, respective single-knockout strains and wt with focus on fruiting-body development. (B) Schematic illustration of the pro22 (Bloemendal et al., 2010) locus before and after homologous integration of the deletion cassette. Primers used for verification of the deletion strain are shown by arrows. PCR fragment sizes are given. (C) Verification of gene deletions in $\Delta$ Smgpil/ $\Delta$ pro22 using PCR. Shown are the calculated fragment sizes for wt and the respective gene deletion. Strains were obtained by crossing the respective single-deletion strains $\Delta$ Smgpi $1 / \mathrm{r} 2$ and $\Delta$ pro22. 
A

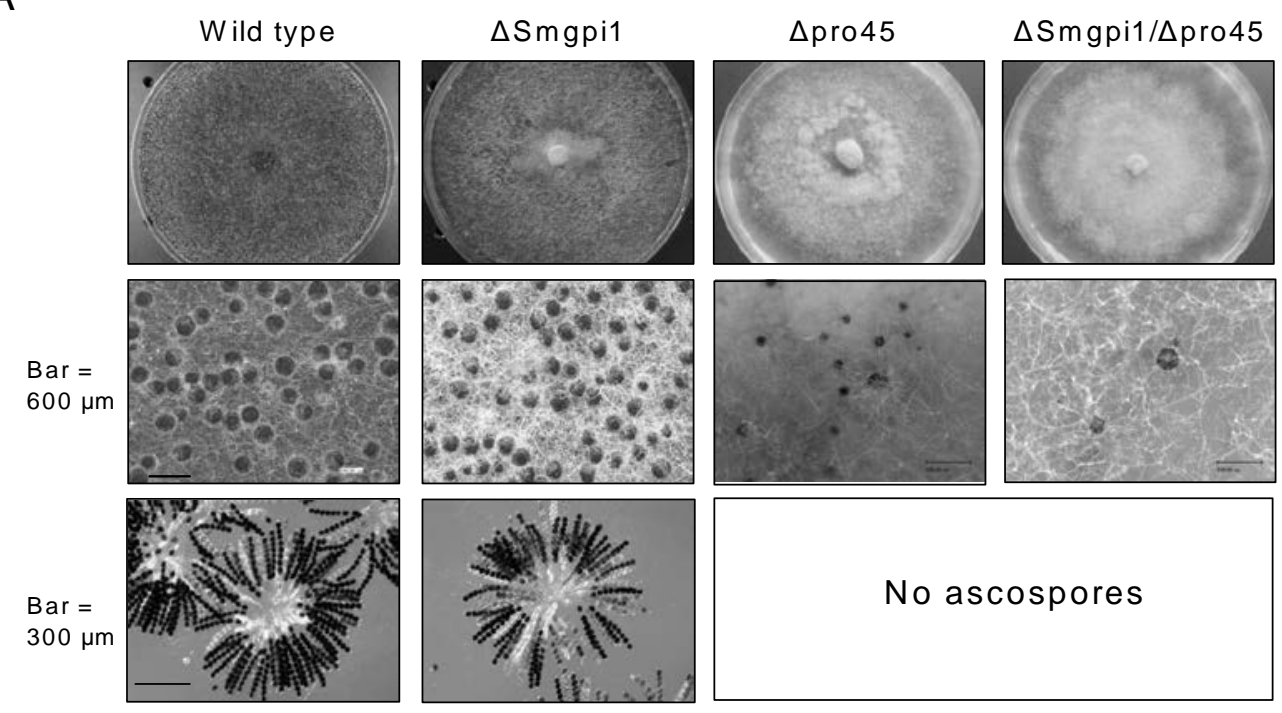

B
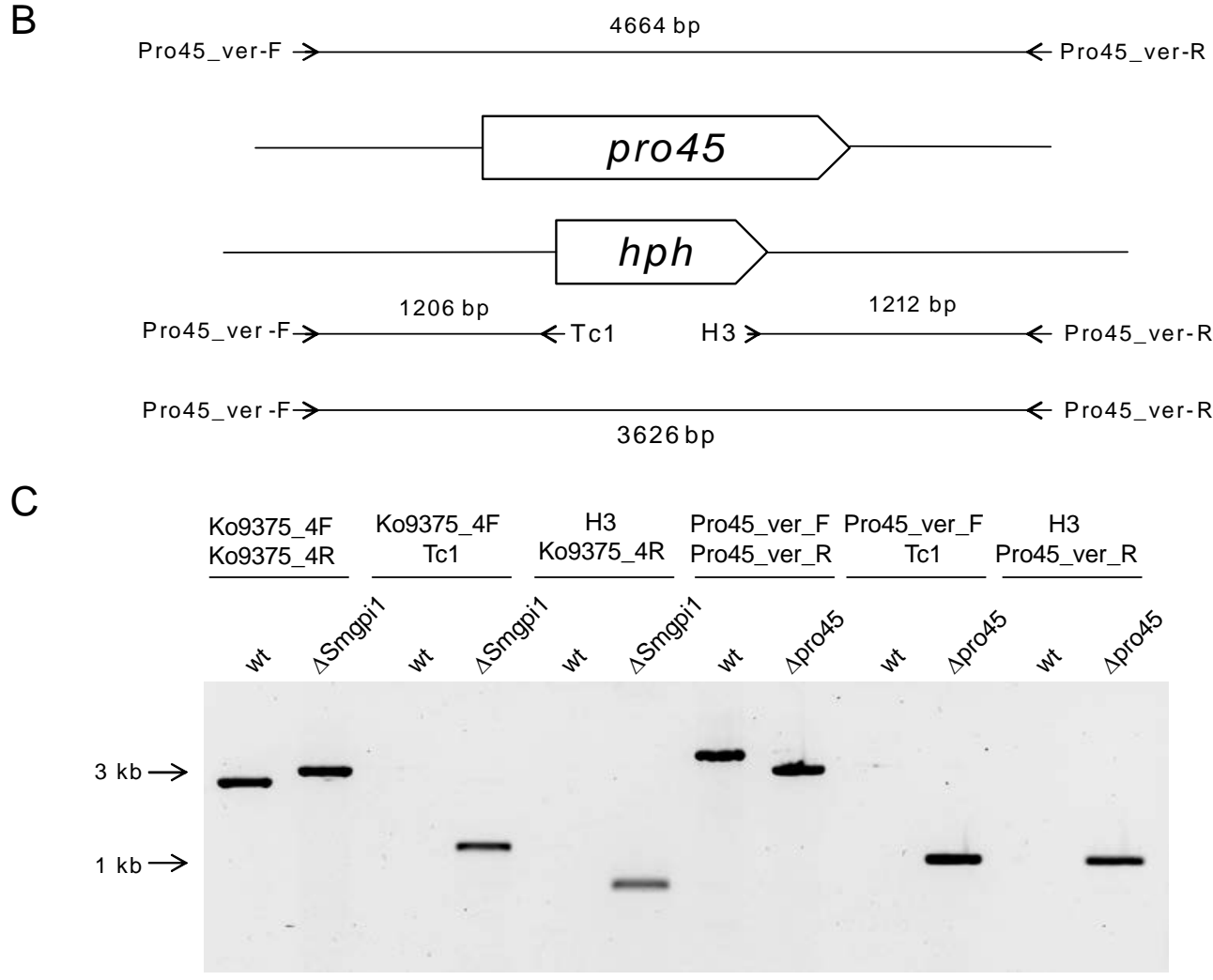

Figure 27. Generation of a $\Delta$ Smgpi $1 / \Delta$ pro45 double-deletion strain. (A) Phenotype of $\Delta$ Smgpi $1 / \Delta$ pro 45 , respective single-knockout strains and wt with focus on fruiting-body development. (B) Schematic illustration of the pro45 locus (Nordzieke et al., 2014) before and after homologous integration of the deletion cassette. Primers used for verification of the deletion strain are shown by arrows. PCR fragment sizes are given. (C) Verification of gene deletions in $\Delta$ Smgpil $/ \Delta$ pro45 using PCR. Shown are the calculated fragment sizes for wt and the respective gene deletion. Strains were obtained by crossing the respective single-deletion strains $\Delta$ Smgpi $1 / \mathrm{r} 2$ and $\Delta$ pro45. 


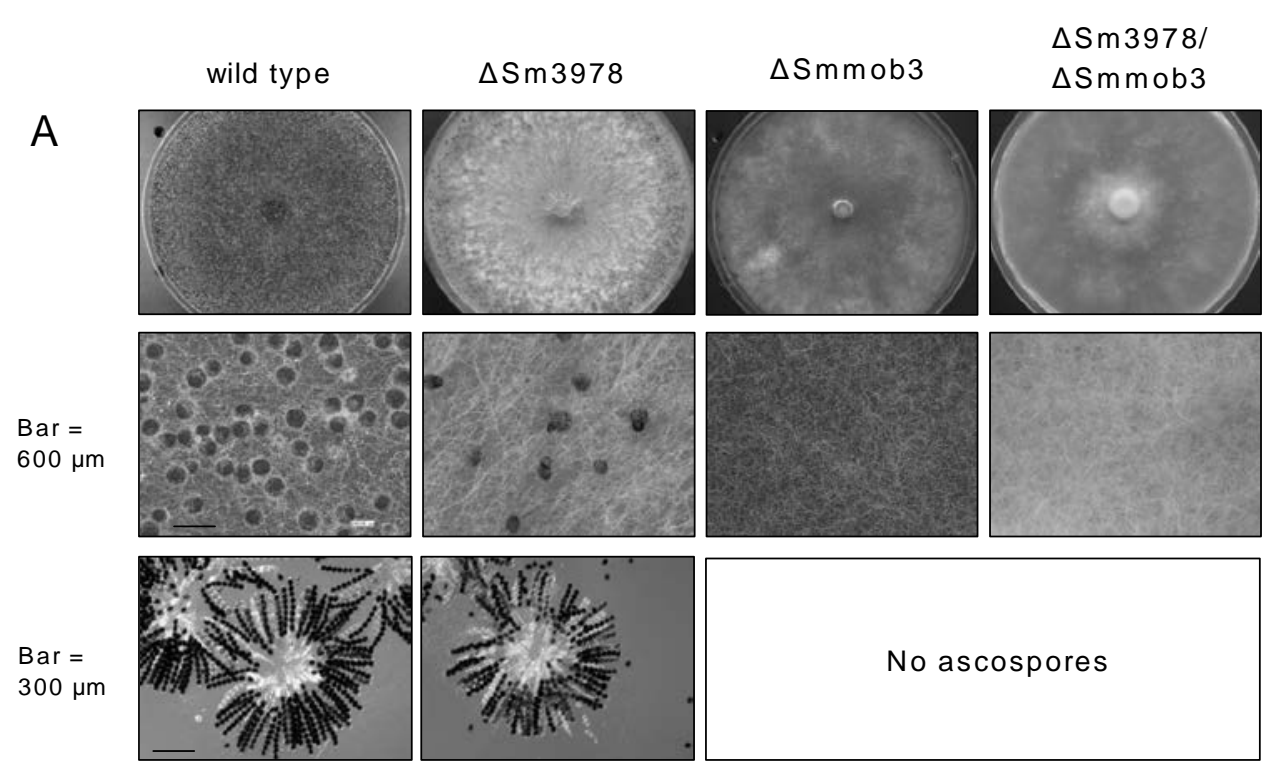

B
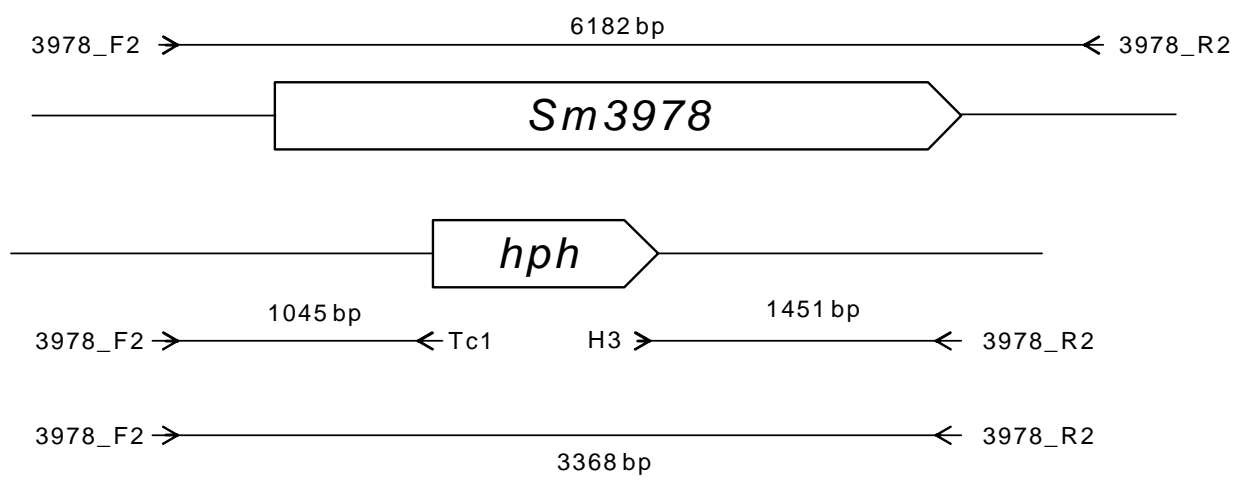

C

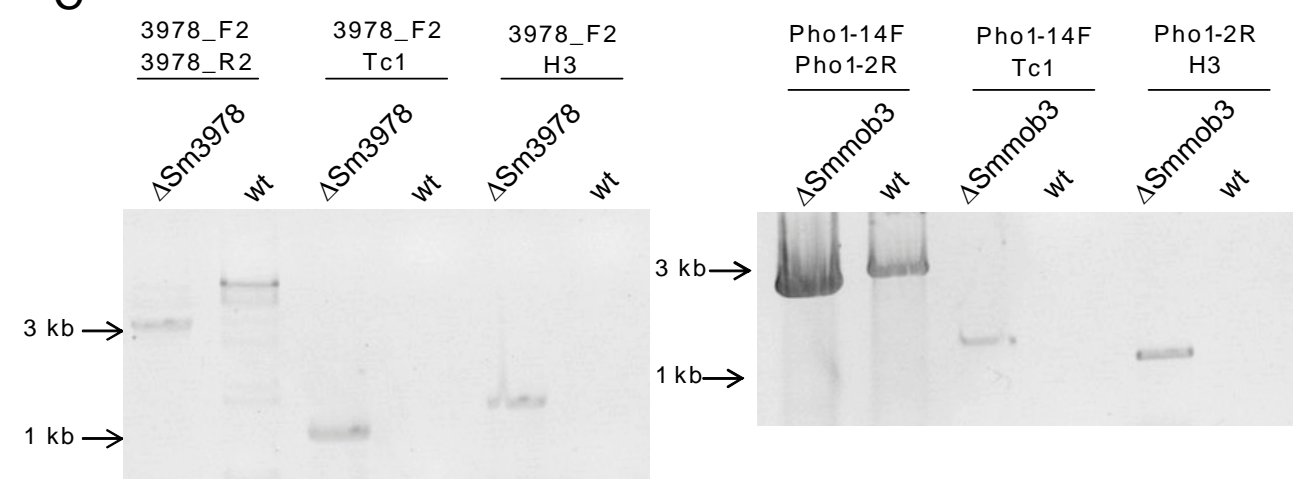

Figure 28. Generation of a $\Delta \mathrm{Sm} 3978 / \Delta \mathrm{Smmob3}$ double-deletion strain. (A) Fruiting body and ascospores development of $\Delta \mathrm{Sm3978} / \Delta \mathrm{Smmob3}$, respective single-knockout strains and wt. (B) Schematic illustration of the $S m 3978$ locus before and after homologous integration of the deletion cassette. Primers used for verification of the deletion strain are shown by arrows. Sizes of PCR fragments are given. (C) Verification of Sm3978 deletion using PCR. Shown are the calculated fragment sizes for wt and the respective gene deletion. Strains were obtained by crossing single deletion strains $\Delta S m 3978$ and $\Delta$ Smmob3/r2. 
Previously, Maerz et al. (2009) demonstrated in N. crassa that MOB3 is essential for hyphal fusion under vegetative growth conditions. Similarly, disruption of Smmob3 impairs hyphal fusion in S. macrospora (Bernhards \& Pöggeler, 2011). These findings raised the question whether the double mutant $\Delta$ Smgpi1/ $\Delta$ Smmob3 underwent hyphal fusion. Hyphal fusion was investigated under two conditions. First, vegetative hyphae of wt, $\Delta$ Smgpi1, $\Delta \operatorname{Smmob} 3$ and $\Delta$ Smgpi1/ $\Delta$ Smmob3 were microscopically observed (Figure 29A). Vegetative hyphae of wt and $\Delta$ Smgpil were capable of hyphal fusion. Hyphal fusion events were detected two days after inoculation.

A
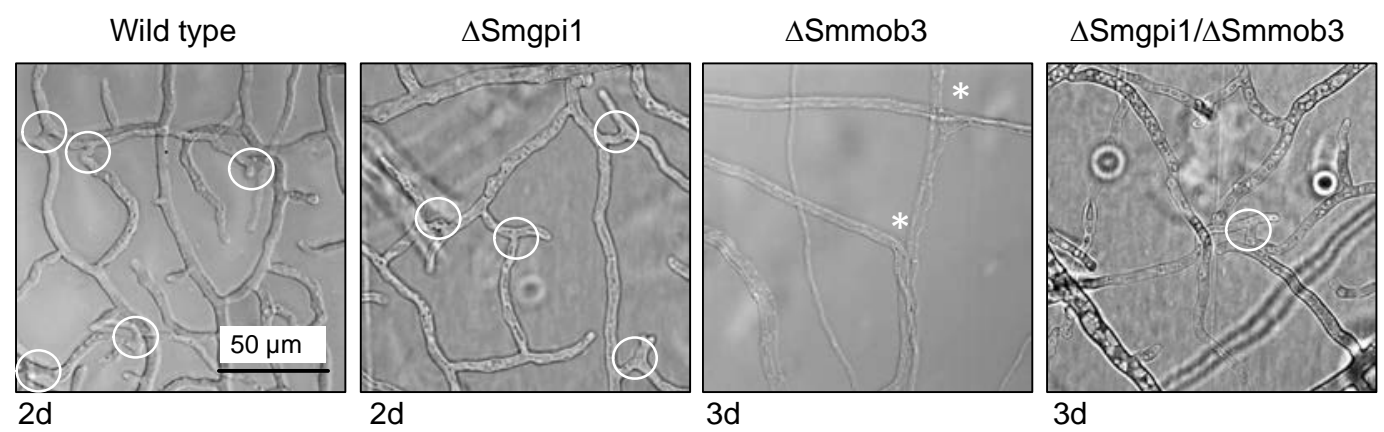

B
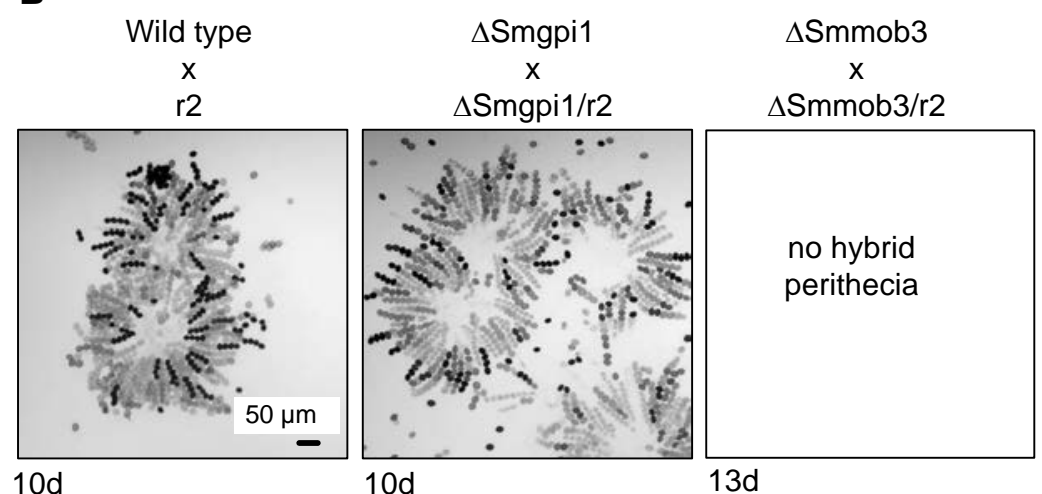

$\Delta$ Smgpi1/ $\Delta$ Smmob3

$\mathrm{X}$ $\Delta$ Smgpi1/ $\Delta$ Smmob3/r2

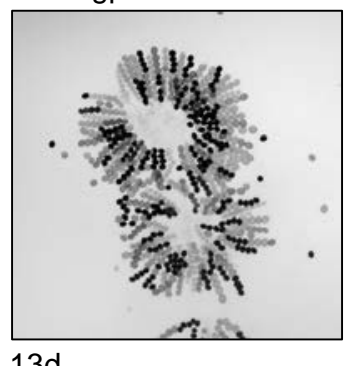

Figure 29. Deletion of Smgpi1 in a sterile $\Delta$ Smmob3 background restores hyphal fusion. (A) Microscopic investigation of hyphal fusion in wt, $\Delta$ Smgpil, $\Delta$ Smmob3 and $\Delta$ Smgpi1/ $\Delta$ Smmob3. Hyphal fusion events are marked with circles; hyphal contacts without fusion are indicated by asterisks. Pictures were taken at subperiphal regions $10 \mathrm{~mm}$ from the growth front. Hyphal fusion was investigated 2-3 days past inoculation. $\Delta$ Smgpi $/ \Delta$ Smmob3 is delayed in hyphal fusion. First fusion was visible after 3 days. (B) Wt, $\Delta$ Smgpi1, $\Delta$ Smmob3 and $\Delta$ Smgpi $1 / \Delta$ Smmob3 were crossed to 2 spore-color mutants with the same deletion background. Crossing perithecia were isolated after 10 days and 13 days past inoculation, respectively. $\Delta$ Smmob3 did not develop fruiting bodies at all. Crossing perithecia of wt, $\Delta$ Smgpil, $\Delta$ Smmob3 and $\Delta$ Smgpi1/ $\Delta$ Smmob3 contain spores allocated in typical 4:4 pattern of successful crossing events of red and black spored strains. 
As described previously, $\Delta$ Smmob3 was impaired in hyphal fusion (Bernhards \& Pöggeler, 2011). Even after $10 \mathrm{dpi}$, no hyphal fusion was visible. The mutant $\Delta$ Smgpi1/ $\Delta$ Smmob3 rarely formed hyphal fusions and did so after a prolonged time of three days. After this time hyphal fusion events were not detectable under the microscope because of extensive aerial hyphae formation. Therefore, recovery of the hyphal fusions in $\Delta$ Smgpil/ $\Delta$ Smmob3 was verified by crosses with spore-color mutants. S. macrospora is a self-fertile fungus that produces perithecia without crossing. Thus, the distinction between self-fertile and hybrid perithecia is difficult. To circumvent this problem, we crossed the spore-color mutant $\mathrm{r} 2$ with the mutant strains $\Delta$ Smgpi1, $\Delta$ Smmob3 and $\Delta$ Smgpi1/ $\Delta$ Smmob3 for red-spored strains $\Delta$ Smgpi1/r2, $\Delta$ Smmob3/r2 and $\Delta$ Smgpi1/ $\Delta$ Smmob3/r2. The mutant $\mathrm{r} 2$ produces red ascospores because of a mutation in a pigment biosynthesis gene (Teichert et al., 2014). Successful hyphal fusion events resulted in black-spored and red-spored asci of hybrid perithecia in the contact zone. The r2 strain, $\Delta$ Smgpi1/r2, $\Delta$ Smmob3/r2 and $\Delta$ Smgpi1/ $\Delta$ Smmob3/r2 were self-crossed with respective blackspored strains (Figure 29). Similar to crosses wt $\mathrm{x}$ r2 and $\Delta$ Smgpi1 $\mathrm{x} \Delta$ Smgpi1/r2, selfing of $\Delta$ Smgpi1/ $\Delta$ Smmob3 $\times \Delta$ Smgpi1/ $\Delta$ Smmob3/r2 resulted in hybrid perithecia with typical 4:4 segregation of black and red spores. No hybrid perithecia were formed after selfing of sterile $\Delta$ Smmob3 strains (Figure 29).

The vegetative growth defect of $\Delta$ Smmob3 was also suppressed in the double-deletion strain $\Delta$ Smgpi $1 / \Delta$ Smmob3. In contrast to the drastically impaired growth of $\Delta$ Smmob3 $(8 \pm 3 \mathrm{~mm} / \mathrm{d})$ the growth of $\Delta$ Smgpi $1 / \Delta \mathrm{Smmob} 3$ was similar to wt ( $25 \pm 5 \mathrm{~mm} / \mathrm{d}$ ) (data not shown).

\subsection{6 $\Delta$ Smgpil forms more fruiting bodies that are small but normally shaped}

To analyze the function of Smgpi1 and the reason for its suppression of $\Delta$ Smmob3, the $\Delta$ Smgpi1 phenotype was studied in detail. Counts of fruiting bodies revealed that $\Delta$ Smgpil produced $152 \% \pm 6.3$ fruiting bodies per $\mathrm{cm}^{2}$ compared to the wt strain, the double-deletion mutant $\Delta$ Smgpi1/ $\Delta$ Smmob3 formed only half of the number of perithecia ( $55 \% \pm 5.9)$ compared to the wt (Figure 30). 


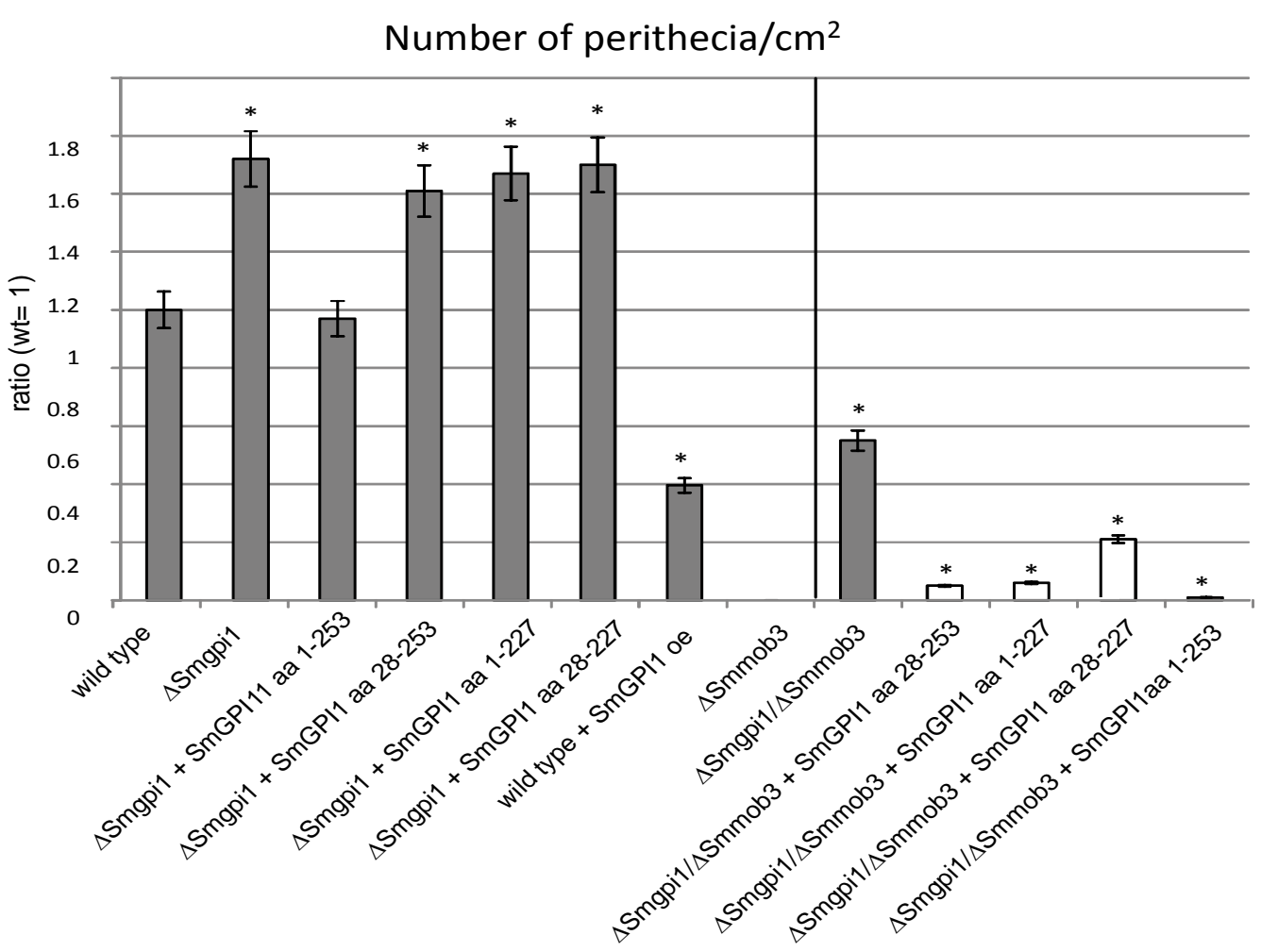

Figure 30. Number of fruiting bodies produced by $\Delta$ Smgpi1, $\Delta$ Smmob3 and $\Delta$ Smgpi1 $/ \Delta$ Smmob3 and complemented strains compared to wt. Wt and $\Delta$ Smgpil were grown for 8 days at $27{ }^{\circ} \mathrm{C}$ on solid SWG medium, $\Delta$ Smgpi $1 / \Delta$ Smmob3 for 14 days, respectively. Data for the respective strains was obtained by counting the number of perithecia produced on an area of $5 \mathrm{~cm}^{2}$ at the edge of the plate. Bars represent the mean value calculated from 10 strains $(n=10)$ for each data set. Ratio of each strain compared to the wt (wt was set 1). Error bars (SD) are given as indicated. Grey bars indicate fertile perithecia containing ascospores; white bars represent sterile perithecia without ascospores. Asterisks indicate significance according to Student's t-test. Thus, values of grey colored bars were compared to wt number of perithecia; white colored bars significance was calculated compared to $\Delta$ Smgpi $1 / \Delta$ Smmob3. P-value $<0.0003$.

The increased number of perithecia returned to wt levels when $\Delta$ Smgpil was complemented by full-length construct SmGPI1 aa 1-253 but not by versions lacking the N-terminal signal sequence (SmGPI1 aa 28-253), the C-terminal processing region for GPI-attachment (SmGPI1 aa 1-227) or both regions (SmGPI1 aa 28-227) (Figure 30). Overexpression of Smgpi1 in wt decreased the total number of produced perithecia per $\mathrm{cm}^{2}$ to $51 \% \pm 3.5$ compared to wt (Figure 30). To determine whether localization of SmGPI1 plays a role to suppresses $\Delta$ Smmob3 sterility, Smgpi1 variants encoding SmGPI1 aa 1-253, SmGPI1 aa 28-253, SmGPI1 aa 1-227 or SmGPI1 aa 28-227 were transformed into $\Delta$ Smgpi1/ $\Delta$ Smmob3 (Figure 30). All $\Delta$ Smgpi1/ $\Delta$ Smmob3 transformants produced a small number of immature perithecia; however, even after prolonged 
incubation, these never contained asci or ascospores. No fruiting bodies were produced from strain $\Delta$ Smgpi1/ $\Delta$ Smmob3 transformed with Smgpi1 full-length. The higher number of fruiting bodies produced by $\Delta$ Smgpil led us to inspect the size and shape of the fruiting bodies in detail. Similar to the wt, $\Delta$ Smgpil produced pear-shaped fruiting bodies; however, they were smaller (Figure 31A). $\Delta$ Smgpil perithecia contained a similar number of asci and ascospores compared to wt (Figure 31B). The decreased size of $\Delta$ Smgpil fruiting bodies was complemented by fulllength Smgpi1 (SmGPI1 aa 1-253), with or without egfp fusion (SmGPI1-eGFP aa 1-492).

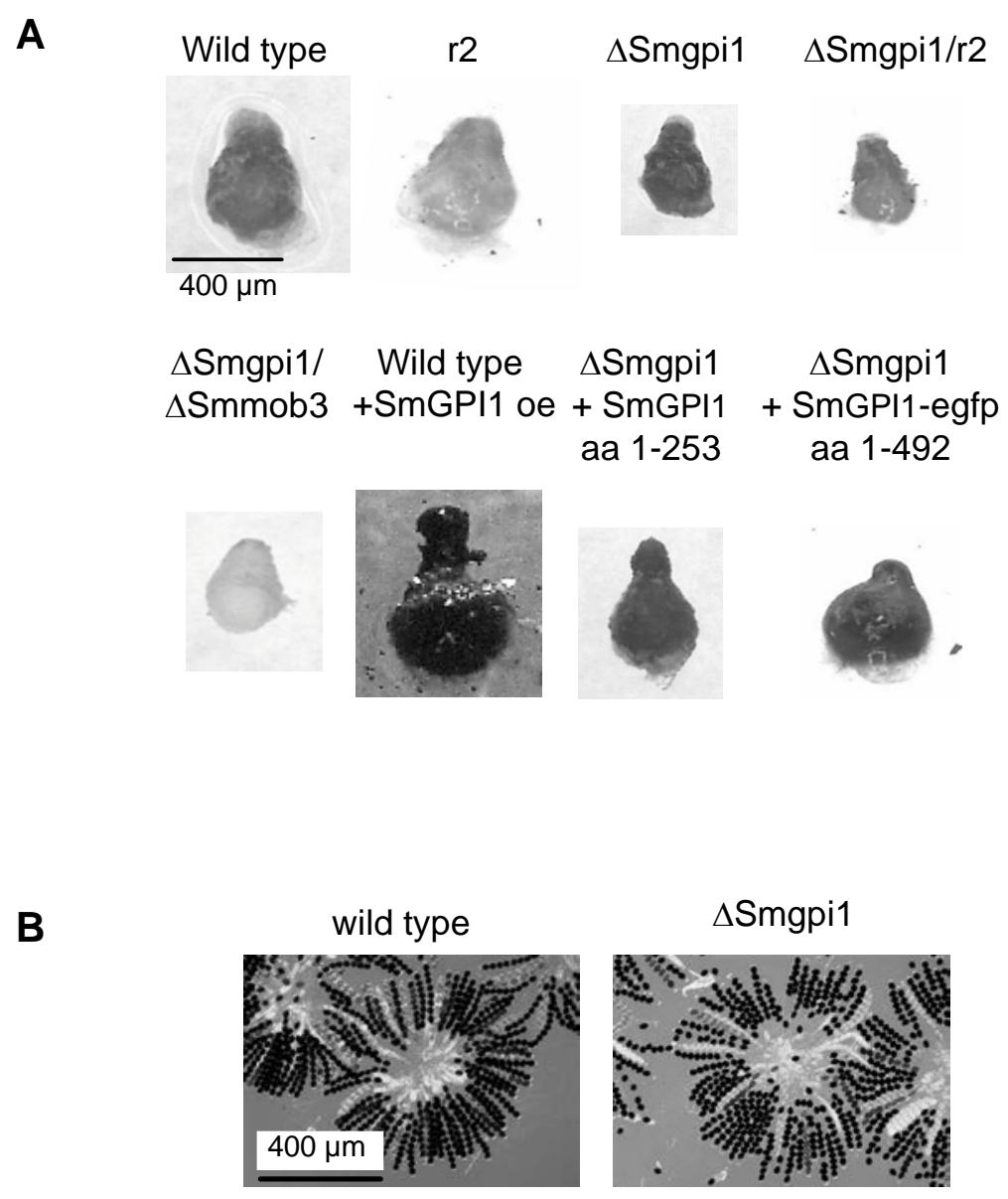

Figure 31. Deletion of Smgpi1 results in smaller mature fruiting bodies. (A) Microscopic analysis of fruiting-body size of wt, $\Delta$ Smgpi1, $\Delta$ Smgpil complemented with the full length Smgpi1 (SmGPI1 aa 1253) or Smgpi1-egfp (SmGPI1 aa 1-492), $\Delta$ Smgpi1/ $\Delta$ Smmob3 and wt expressing an additional copy of Smgpi1. Fruiting bodies were isolated at day 8 after inoculation. Complementation of the gene deletion restores normal size of fruiting bodies. (B) Ascus rosette from wt perithecium compared to perithecium of $\Delta$ Smgpi1. Both strains produce an equal number of asci and ascospores. 
Truncated versions of Smgpi1 (SmGPI1 aa 28-253, 1-227 and 28-227) did not restore fruitingbody size (data not shown). Quantitative analysis revealed a different distribution of fruitingbody diameters in the deletion mutant $\Delta$ Smgpil (Figure 32). Compared to wt, $\Delta$ Smgpil developed more mature fruiting bodies with a diameter of 0.2-0.3 $\mathrm{mm}$ and fewer with a diameter of $>0.4 \mathrm{~mm}$. This effect was complemented by full-length Smgpi1. Overexpression of Smgpi1 in wt slightly increased perithecia diameter (Figure 32). Fruiting bodies in the double-deletion strain $\Delta$ Smgpi $1 / \Delta$ Smmob3 were slightly smaller than in $\Delta$ Smgpi1 (Figure 31).

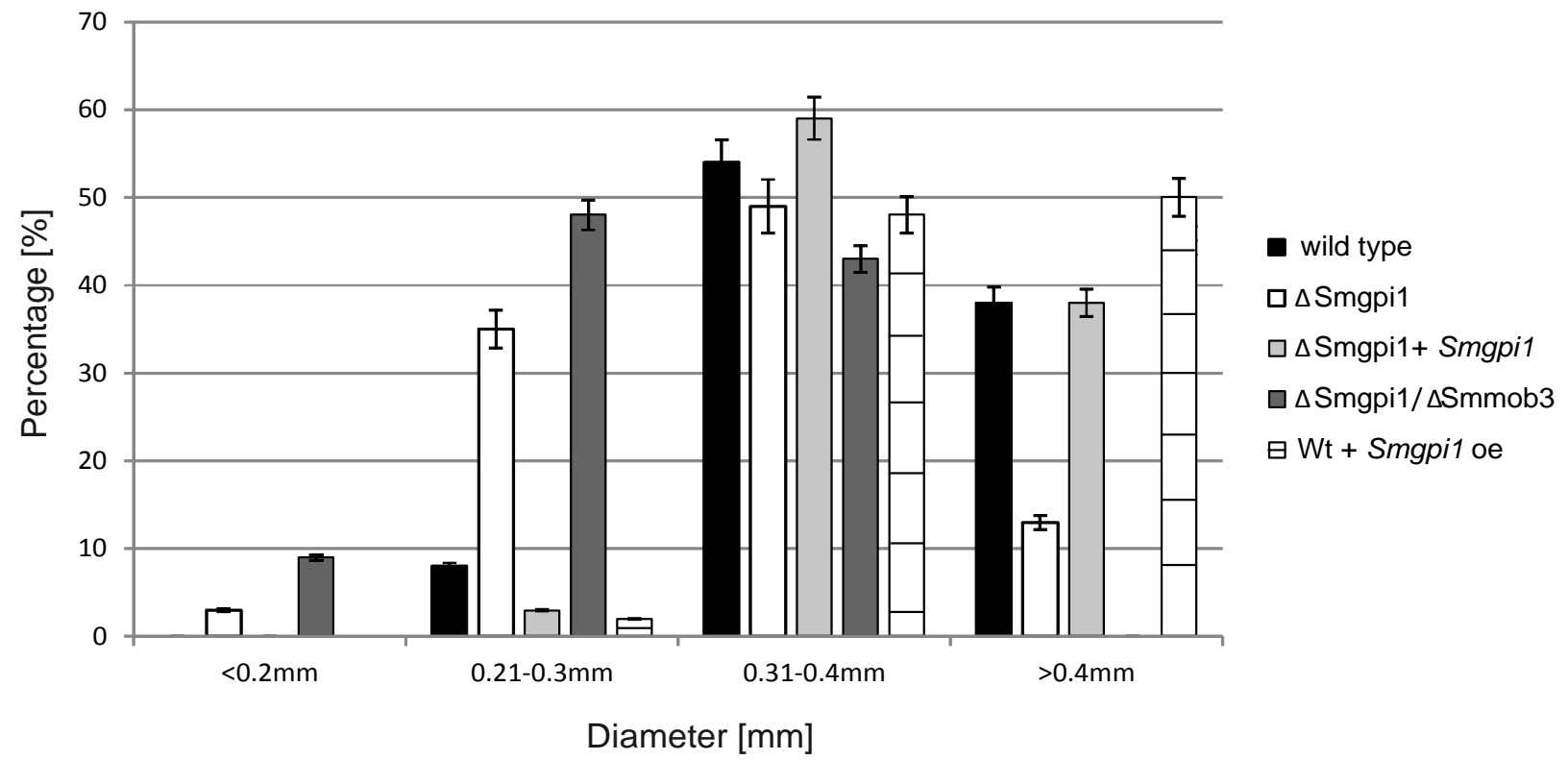

Figure 32. Quantitative evaluation of perithecia size (diameter) from wt, $\Delta$ Smgpil and $\Delta$ Smgpi1 expressing the full length Smgpi1 (SmGPI1 aa 1-253), $\Delta$ Smgpi1/ $\Delta$ Smmob3 and wt expressing an additional copy of Smgpi1. Bars represent the mean value calculated from data obtained from 100 measured fruiting bodies of three biological replicates, respectively. Error bars (SD) are given as indicated $(\mathrm{n}=100)$. Scale bar as indicated. 


\subsection{The GCKs SmKIN3 and SmKIN24}

\subsubsection{S. macrospora encodes two kinases similar to the mammalian STRIPAK-associated kinases STK24, STK25, MST4 and MINK1.}

In mammals three members of the GCK III subfamily of the Ste20 kinases (STK24, STK25, and MST4); and one member of the GCK IV family (Mishappen-like kinase 1 , MINK1) are associated with the STRIPAK complex (Goudreault et al., 2009, Hyodo et al., 2012). To date, no STRIPAK-associated kinases have been identified in filamentous fungi so far.

Therefore, we performed a BLASTP search using mammalian kinases STK24, STK25, MST4 and MINK1 as query against the $S$. macrospora proteome (http://blast.bemd.ncbi.nlm.nih.gov/(Nowrousian, 2010)) to identify putative S. macrospora homologs of the mammalian kinases. This search revealed the putative germinal center kinases SMAC_01456 (F7VQV9) and SMAC_04490 (F7VYS5) to be orthologous to the mammalian GCKs (Table 6).

Table 6. BLASTP search of the human STRIPAK associated GC kinases against the $S$. macrospora proteom

\begin{tabular}{rll}
\hline \multicolumn{1}{c}{ Type } & $\begin{array}{c}\text { Human } \\
\text { (accession number) }\end{array}$ & \multicolumn{1}{c}{$\begin{array}{c}\text { S. macrospora } \\
\text { best hit }(\mathrm{e}-\mathrm{value})\end{array}$} \\
\hline GCK III & MST3, STK24 & SMAC_01456 $\left(5 \mathrm{e}^{-136}\right)$ \\
& (Q9Y6E0.1) & SMAC_04490 $\left(9 \mathrm{e}^{-126}\right)$ \\
GCK III & MST4,MASK & SMAC_01456 $\left(4 \mathrm{e}^{-136}\right)$ \\
& (Q9P289.2) & SMAC_04490 $\left(6 \mathrm{e}^{-125}\right)$ \\
GCK III & STK25, SOK1, YSK1 & SMAC_01456 $\left(3 \mathrm{e}^{-134}\right)$ \\
& (O00506.1) & SMAC_04490 $\left(4 \mathrm{e}^{-123}\right)$ \\
GCK IV & MINK1 & SMAC_01456 $\left(8 \mathrm{e}^{-75}\right)$ \\
& $($ NP_722549.2) & SMAC_04490 $\left(2 \mathrm{e}^{-69}\right)$
\end{tabular}

The open reading frame (ORF) of SMAC_04490 comprises 2758 bp and contains three putative introns: 96 bp at position 53-148, 117 bp at position 217-333 and 82 bp at position 502-583 (Figure 34). The calculated molecular weight (MW) of the encoded 820 aa protein is $91.4 \mathrm{kDa}$ with an isoelectric point (pI) of 9.47. It showed a high sequence identity to the serine/threonineprotein kinase 3 of Neurospora crassa (locus tag NCU04096, gene symbol prk-9) (Figure 33) and was therefore named SmKIN3. 

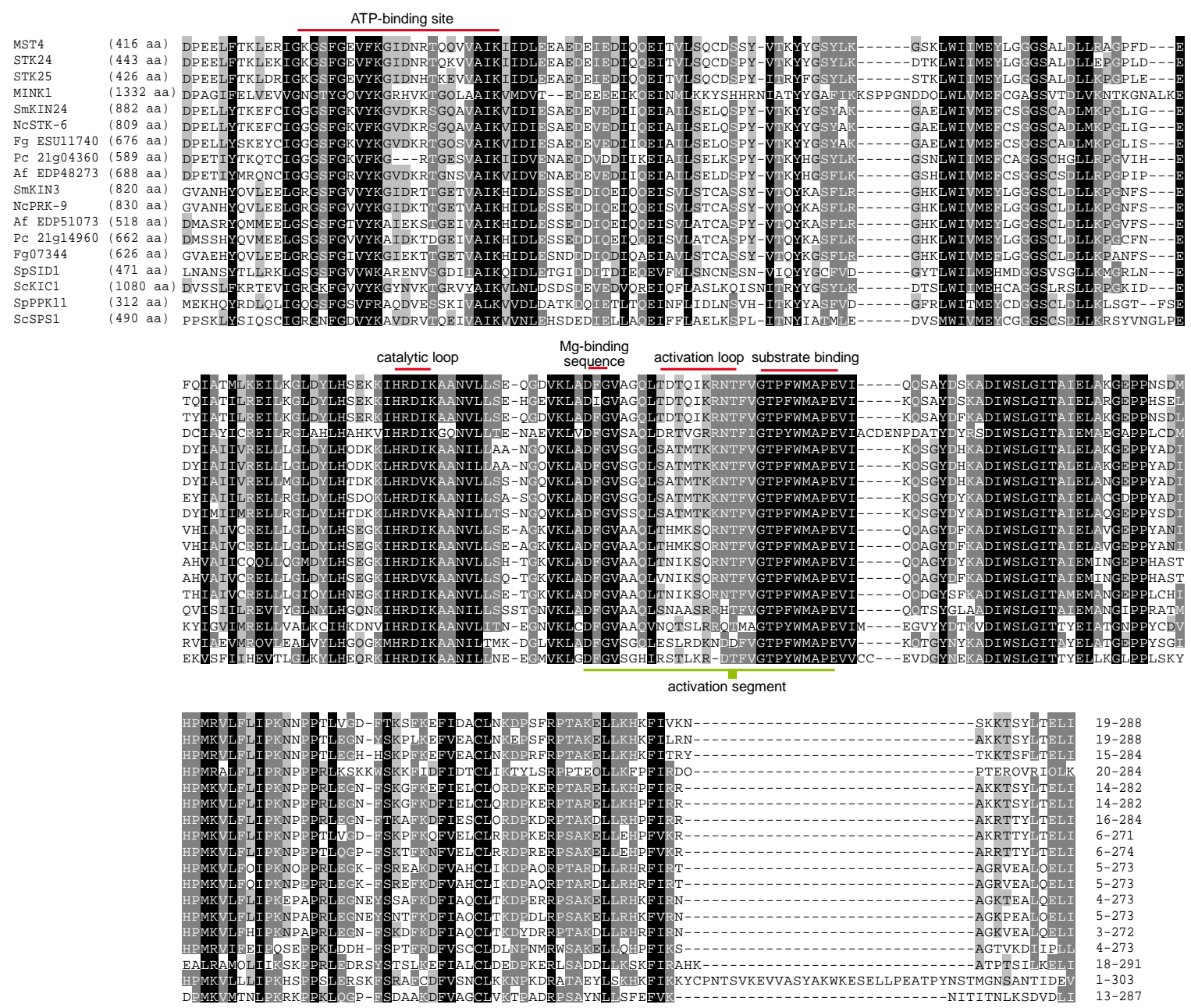

Figure 33. Multiple sequence alignment and aa identity of mammalian kinases identified as STRIPAK members with putative homologues from Ascomycota. Protein sequences were aligned with ClustalX2 (Larkin et al., 2007) and visualized with GeneDoc (Nicholas et al., 1997). MST4, mammalian STE20-like protein kinase 4 (Accession number: Q9P289); STK24, mammalian STE20-like protein kinase 3 (Q9Y6E0); STK25, mammalian serine/threonine-protein kinase 25 (O00506); MINK1, mammalian misshapen-like kinase 1 (Q8N4C8); SmKIN24, putative serine/threonine-protein kinase 24 from S. macrospora (F7VQV9), NcSTK-6, serine/threonine-protein kinase 24 from $N$. crassa (V5IQF9); Fg ESU11740, serine/threonine-protein kinase 24 from F. graminearum (I1RNY7); Pc21g04360, putative STE20-like protein kinase from P. chrysogenum (B6HMA9); Af EDP48273, putative STE20-like kinase from A. fumigatus (BA78_1793), SmKIN3, putative serine/threonine-protein-kinase 3 from $S$ macrospora (F7VYS5); NcPRK-9, serine/threonine-protein-kinase 3 from N. crassa (V5INC1); Af EDP51073, putative Ste20-like kinase from A. fumigatus (B0Y2A3); Pc21g14960, putative serine/threonine-protein kinase from P. crysogenum; (B6HJ11); Fg07344, putative serine/threonineprotein kinase from F. graminearum (A0A016PPL3); SpSID1, serine/threonine-protein kinase from Schizosaccharomyces pombe, (O14305); ScKIC1, serine/threonine-protein kinase from S. cerevisiae, (P38692); SpPPK11, serine/threonine-protein kinase from S. pombe, (O14047); ScSPS1, sporulationspecific protein 1 from S. cerevisiae (P08458). Total numbers of aa are given in brackets. Regions of the aligned $\mathrm{N}$-terminal domain of the protein sequences are indicated in brackets at the end of the alignment. 
ATP-binding side, catalytic loop, Mg-binding sequence, activation loop and region for substrate binding are marked with red lines. The entire activation segment is underlined in green. Filled box indicate the threonine residue (Thr190 in in MST4, Thr182 in STK24, and Thr174 in STK25), phosphorylated during activation. Total numbers of aa are given in brackets at the beginning of the alignment. Aligned $\mathrm{N}-$ terminal domains of the protein sequences are given at the end of the alignment.

Two variants have been reported for $N$. crassa NCU04096 protein: a long version resulting from a transcript after splicing of three introns, and a short version derived from a transcript retaining intron 3 and following initiation of translation at a down-stream ATG codon (http://www.broadinstitute.org/annotation/genome/neurospora/). The position of all three introns is conserved in Smkin3 and prk-9, but we identified splicing of all three introns in Smkin3 using RT-PCR and cDNA sequencing, and splicing of intron 3 did not appear to be optional (Figure 34). In addition, the position of the down-stream start codon is conserved in Smkin3. Initiation of translation from this ATG codon would lead to an N-terminally truncated SmKIN3 version of 663 aa with an MW of $74.3 \mathrm{kDa}$. The second ORF (SMAC_01456) encoding a putative homolog of mammalian GC III and GC IV kinases, encompasses 2947 bp and is predicted to be disrupted by four introns: 107 bp at position 77-183, 76 bp at position 215-290, 51 bp at position 608-658 and 64 bp at position 2413-2476. The calculated MW of the encoded protein of 882 aa is 98.3 $\mathrm{kDa}$ with an isoelectric point (pI) of 7.70.The closest homolog of this protein was serine/threonine-protein kinase 24 of $N$. crassa (locus tag NCU00772, gene symbol stk-6 alias mst-1). The $S$. macrospora protein was therefore designated SmKIN24. 


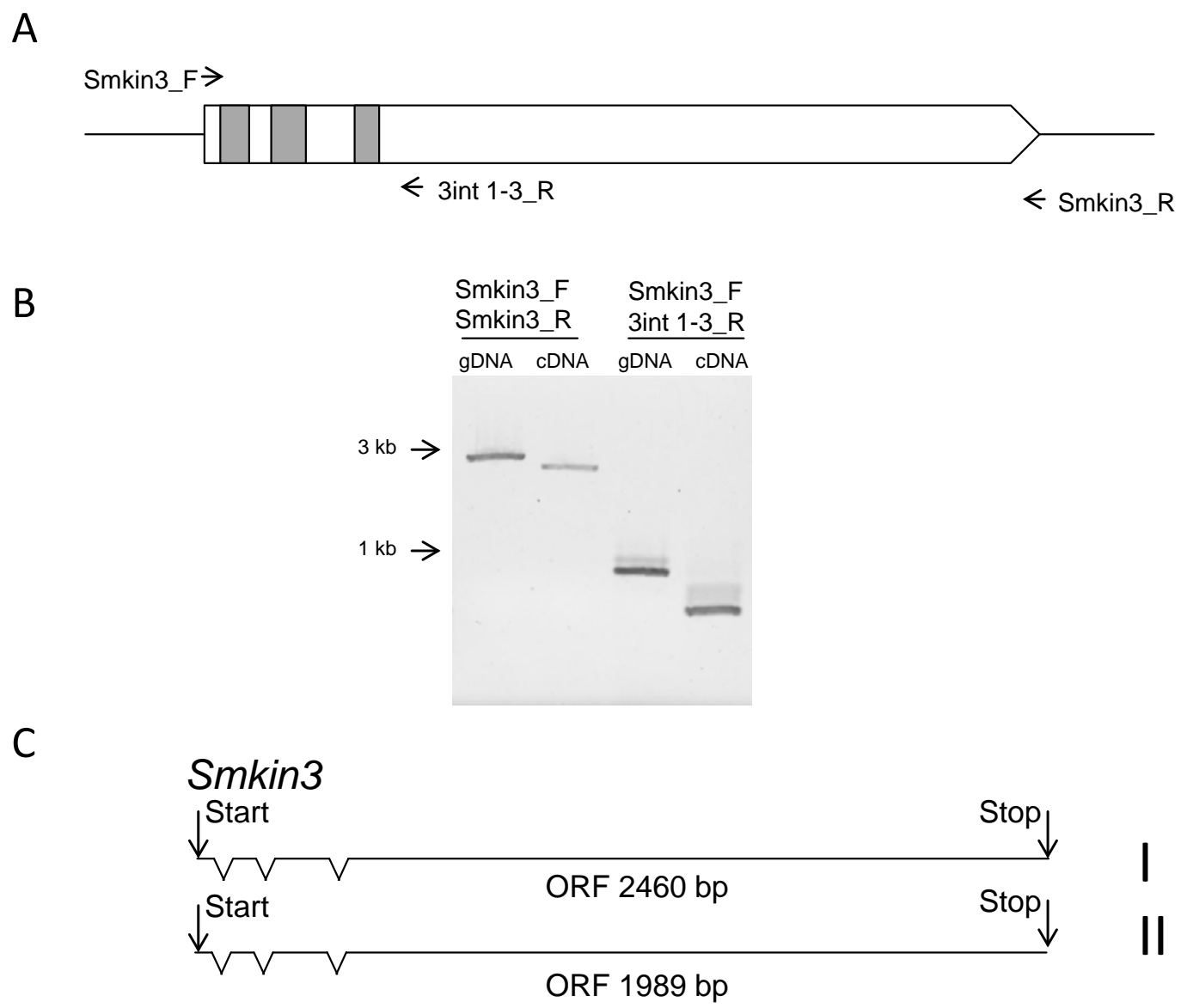

Figure 34. RT-PCR analysis of Smkin3. (A) Schematic illustration of Smkin3. Introns are indicated as grey boxes, positions of primers used for analysis of intron splicing are indicated by arrows. (B) Results of the RT-PCR analysis. Shown are the obtained amplicons for respective primer pairs from cDNA and gDNA. (C) Schematic illustration of Smkin3 transcripts.

Similarly to NCU04096, two gene products have been identified for NCU00772: a long version resulting from splicing of three introns at same positions as the first three introns of Smkin24, and a short version resulting from skipping of intron 1 and translation initiation at a down-stream ATG start codon. This downstream start codon is conserved in Smkin24. RT-PCR and cDNA sequencing of Smkin24 revealed that, like NCU0772, splicing of the first intron was optional. Additionally, sequencing of full length RT-PCR products showed optional splicing of intron 4, which is not present in NCU0772 (Figure 35). 
A

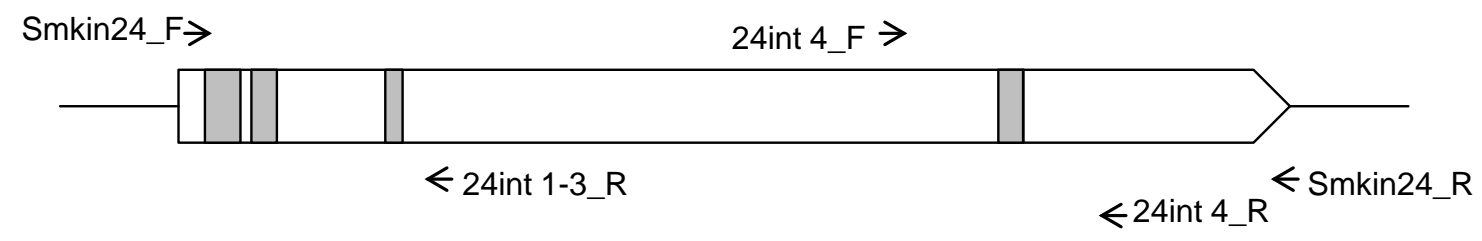

B

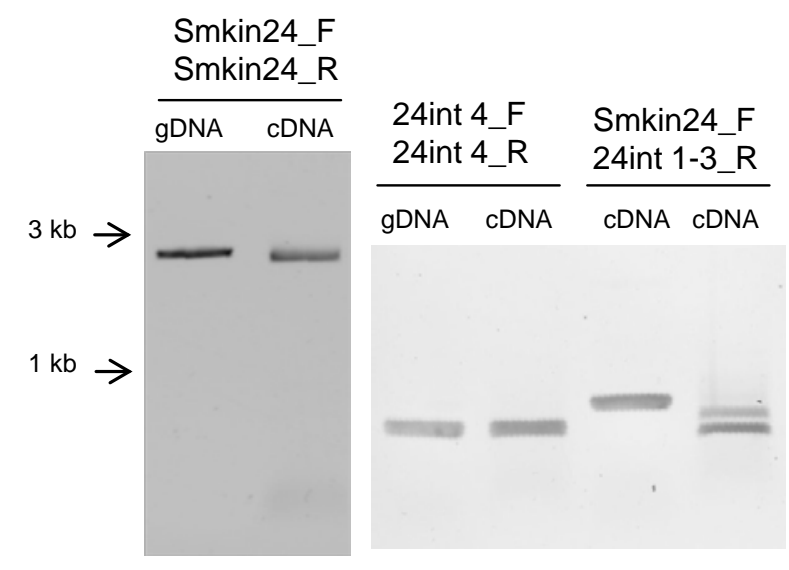

C

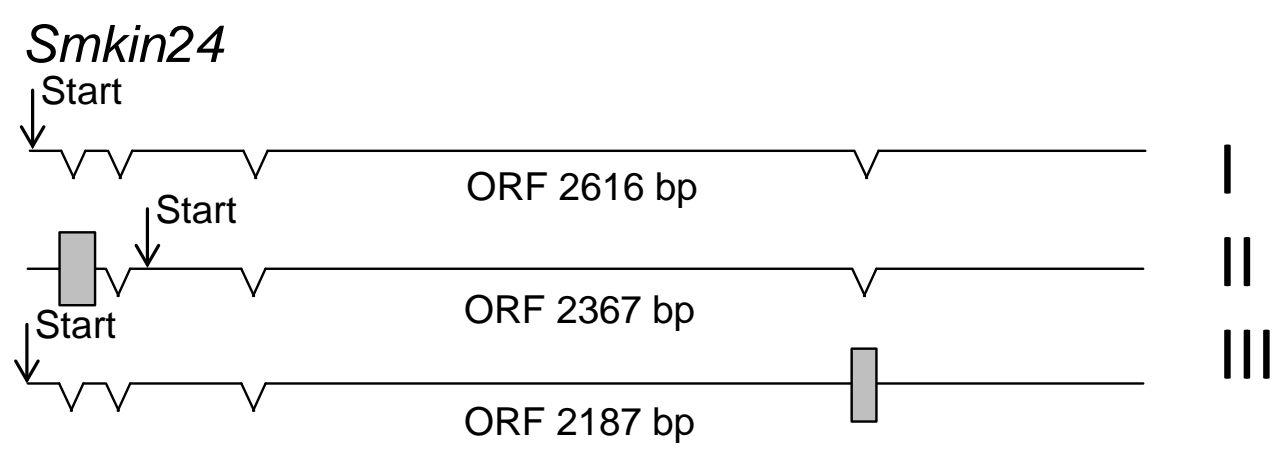

Figure 35. RT-PCR analysis of Smkin24. (A) Schematic illustration of Smkin24. Introns are indicated as grey boxes, positions of primers used for analysis of intron splicing are indicated by arrows. (B) Results of the RT-PCR analysis. Shown are the obtained amplicons for respective primer pairs from cDNA and gDNA. (C) Schematic illustration of Smkin24 transcripts identified by cDNA sequencing.

Thus, in addition to the full-length protein two further variants are encoded by Smkin24. Skipping the splicing of intron 1 and initiation of translation at the downstream ATG codon would result in a 789 aa protein with a MW of $88 \mathrm{kDa}$, whereas skipping intron 4 splicing would lead to a C-terminal truncated version of 729 aa with a MW of $82 \mathrm{kDa}$ (Figure 36). 
Figure 36. Alignment of aa sequences encoded by alternatively spliced Smkin24 transcripts. Smkin24 without introns results in a protein of 882 aa and thus representing the largest protein. Expression of Smkin24 with remaining intron I but removed intron II-IV results in a protein comprising aa 94-882 of the protein derived from Smkin24 without introns. Smkin24 with spliced intron I-III but remaining intron IV results in a protein comprising aa 1-729. The numbers I-III at the beginning of the aa acid sequences for each Isoform refers to Figure 35.

In mammalian GC III and IV kinases the regulatory domain lies C-terminal to the catalytic domain and is heterogeneous in sequence (Record et al., 2010). A search for conserved domains at CCD http://www.ncbi.nlm.nih.gov/Structure/cdd/wrpsb.cgi (Marchler-Bauer et al., 2011) revealed that both SmKIN3 and SmKIN24 exhibit a typical STKc MST3-like catalytic domain of mammalian Ste20-like kinase 3 serine/threonine-protein kinases (accession cd06609, E-value SmKIN3 $=1.13 \mathrm{e}^{-177}$, E-value SmKIN24 $=0.0$ ) at the N-terminal part of the protein. As shown in 
Figure 33, SmKIN3 and SmKIN24 possess a conserved ATP-binding site [ $\underline{G}$ X $\underline{G} X(F) \underline{G} X_{16} \underline{K}$ ], a catalytic loop sequence [HRDIK], a $\mathrm{Mg}^{2+}$-coordinating sequence [DFG], a substrate binding motif [GTPFWMAPE] and a critical threonine residue, phosphorylated during activation, at the end of the activation loop (Sugden et al., 2013, Delpire, 2009). Based on an aa alignment of the $\mathrm{N}$-terminal catalytic domain, S. macrospora SmKIN3 and SmKIN24 share a high level of sequence identity with the N-terminus of MST4 (67\%/67\%), STK24 (68\%/68\%), STK25 (67\%/68\%) and MINK1 (46\%/46\%). However, the C-terminally located regulatory domains are structurally different to GCKs from other filamentous ascomycetes, as well as S. cerevisiae and S. pombe (less than 20\%) (Figure 37).

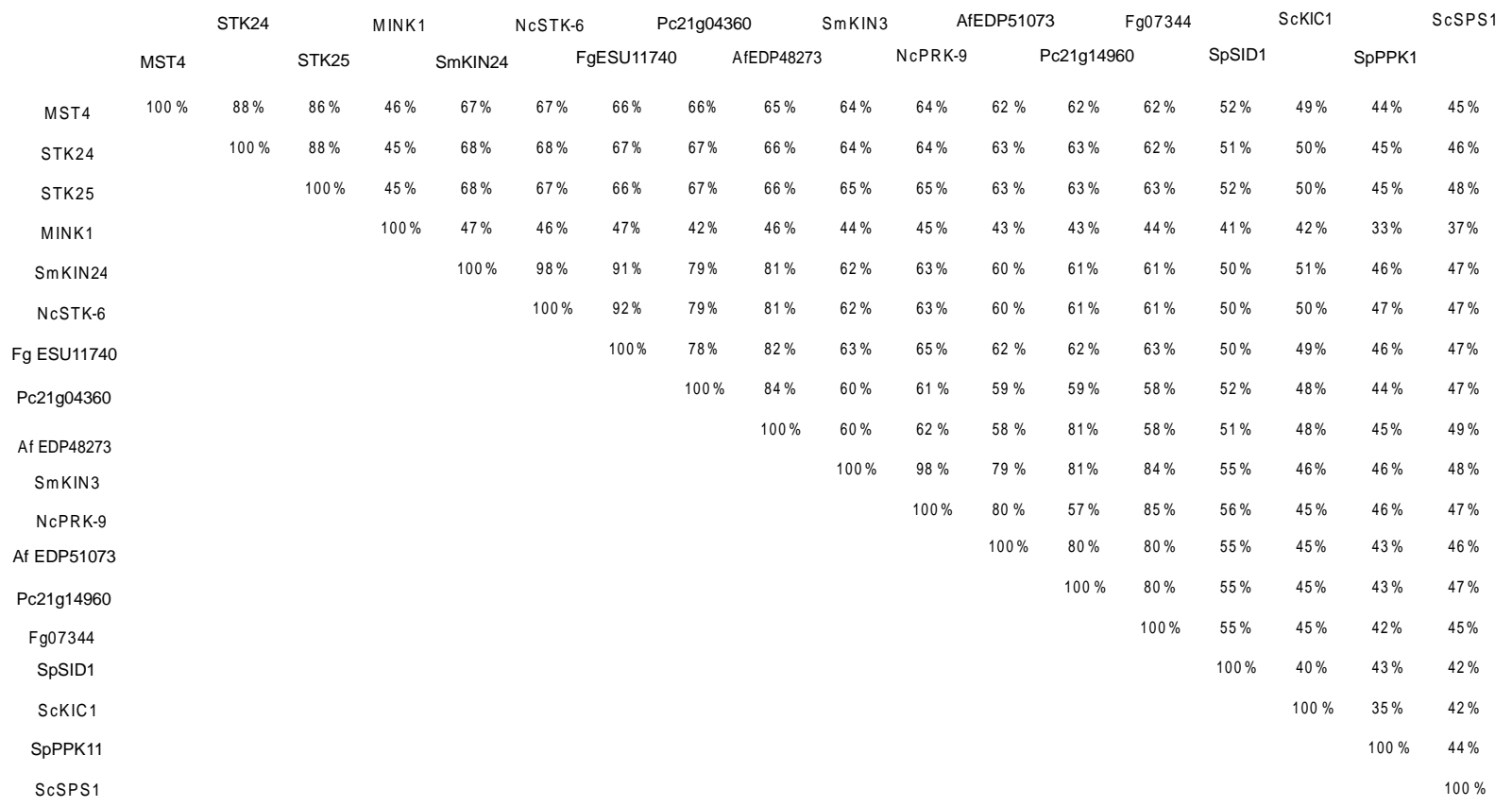

Figure 37. Identity of aligned aa sequences of mammalian kinases identified as STRIPAK members with putative homologues from Ascomycota in pair-wise comparison.

Phylogenetic analysis revealed that SmKIN3 and SmKIN24 were strictly separated and cluster together with putative homologs from other filamentous ascomycetes (Figure 38). 
filamentous ascomycetes Sm KIN24-like

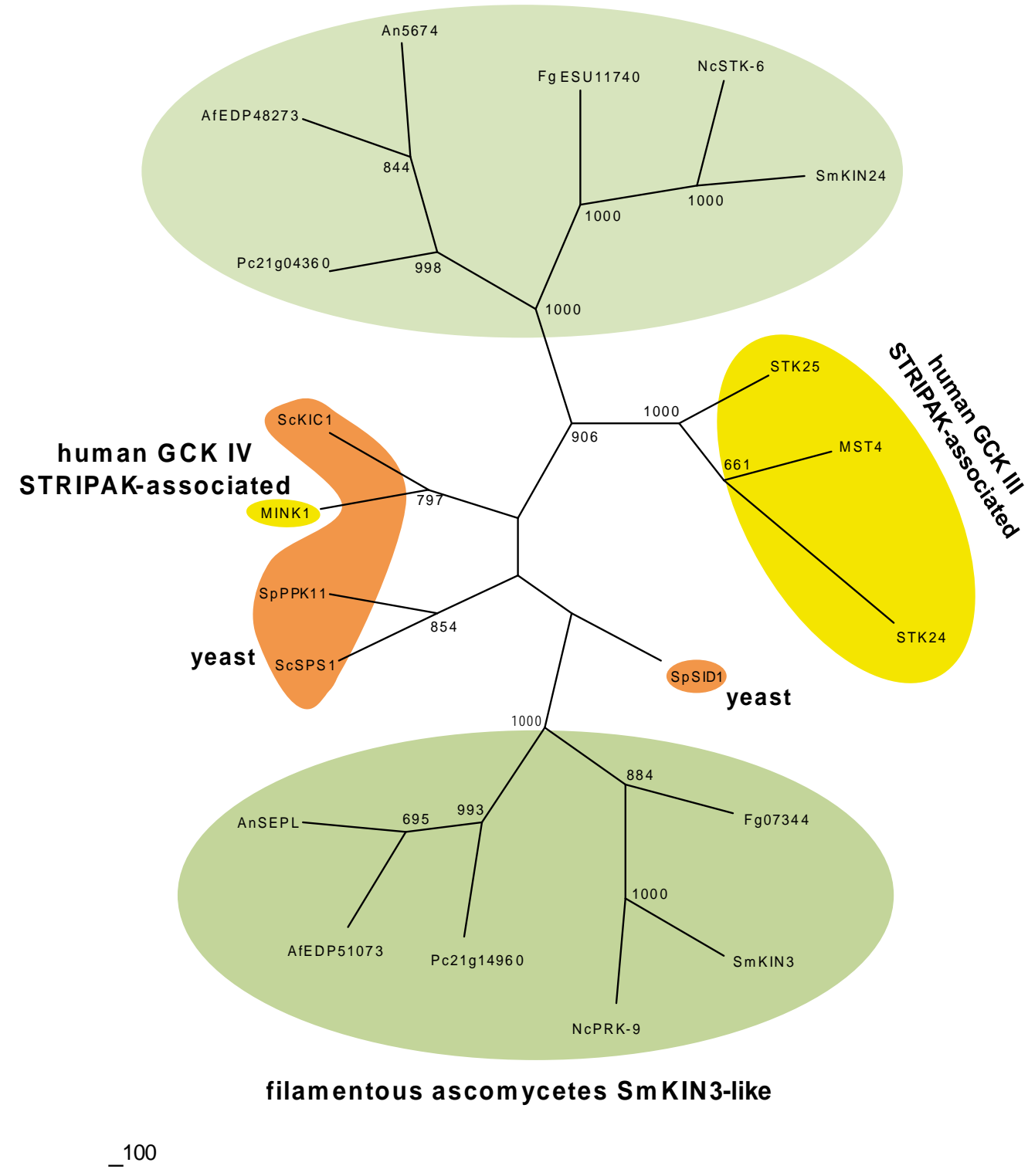

Figure 38. Unrooted neighbor-joining tree of human GCKs MST4, STK24, STK25, MINK1 and their orthologs in ascomycetes. Orthologs were identified by BLAST search using the listed human GCKs as template. Catalytic domains proteins were aligned with ClustalX2 (Larkin et al., 2007). The phylogenetic tree was made with programs included in PHYLIP 3.695 (Felsenstein, 2013). The human GCKs are displayed in yellow, the orthologs from the yeasts $S$. cerevisiae and $S$. pombe in orange. KIN3-like kinases and KIN24-like kinases from filamentous ascomycetes are shown in light green. The respective accession numbers of the proteins are given in Figure 33 except of the Aspergillus nidulans kinases AnSEPL (C8V5Z7) and An56574 (Q5B1A6).. The tree is divided into four branches, comprising the human GCKs MST4, STK24, and STK25, the KIN24-like group of filamentous ascomycetes, the KIN3like group of filamentous ascomycetes, and MINK1 with SPS1 and KIC1 from S. cerevisiae as well as S. pombe PPK11 from. SID1 from S. pombe is separately positioned, near the SmKIN3-like group. The numbers at the nodes indicate bootstrap support. 
Mammalian GC III kinases contain putative nuclear export signals (NES) and bipartite nuclear localization sequences (NLS) (Lee et al., 2004). A putative NES at aa position 493-509 was predicted for SmKIN3 by NetNES1.1 (la Cour et al., 2004) and a bipartite NLS by the program cNLS (Kosugi et al., 2009) for SmKIN3 at position 463-490 (score 4.1) and SmKIN24 at position 783-817 (score 3.8).

\subsubsection{SmKIN3 interacts physically with PRO11}

Striatin is the scaffold of mammalian STRIPAK complex kinases MST4, STK24, STK25 and MINK1 (Hyodo et al., 2012, Goudreault et al., 2009). PRO11 was previously shown to be the $S$. macrospora homolog of mammalian Striatin (Pöggeler \& Kück, 2004). The sequence similarity between SmKIN3 and SmKIN24 and the mammalian GC III and GC IV kinases (Figure 36) led us to inspect whether both S. macrospora kinases can interact with PRO11 in a Y2H system. Full-length cDNAs of Smkin3 and Smkin24 were cloned into the Y2H vector pGBKT7 that contains the GAL4 DNA-binding domain. Plasmid pAD11FL encoding full-length PRO11 served as prey vector. Plasmids were transformed into yeast strains Y187 (pGBKT7 constructs) or AH109 (pGADT7 constructs). Strains carrying pBD-SmKIN3 and pBD-SmKin24 plasmids were checked for transactivation activity by mating with yeast strain AH109 containing pGADT7. A strain carrying pGBKT7 and pGADT7 was used as negative control. Expression of GAL4 fusion proteins from pBD-SmKIN3 and pBD-SmKIN24 was checked by interaction with the yeast protein RanBPM (Tucker et al., 2009). Previously, we showed that the Mob domain protein SmMOB3, the mammalian phocein homologue, is a strong interaction partner of PRO11 (Bloemendal et al., 2012, Bernhards \& Pöggeler, 2011). We therefore tested the interaction of both kinases with SmMOB3. Using the Y2H system we confirmed that both SmKIN3 and SmKIN24 could physically interact with PRO11 but not with SmMOB3 (Figure 39). 

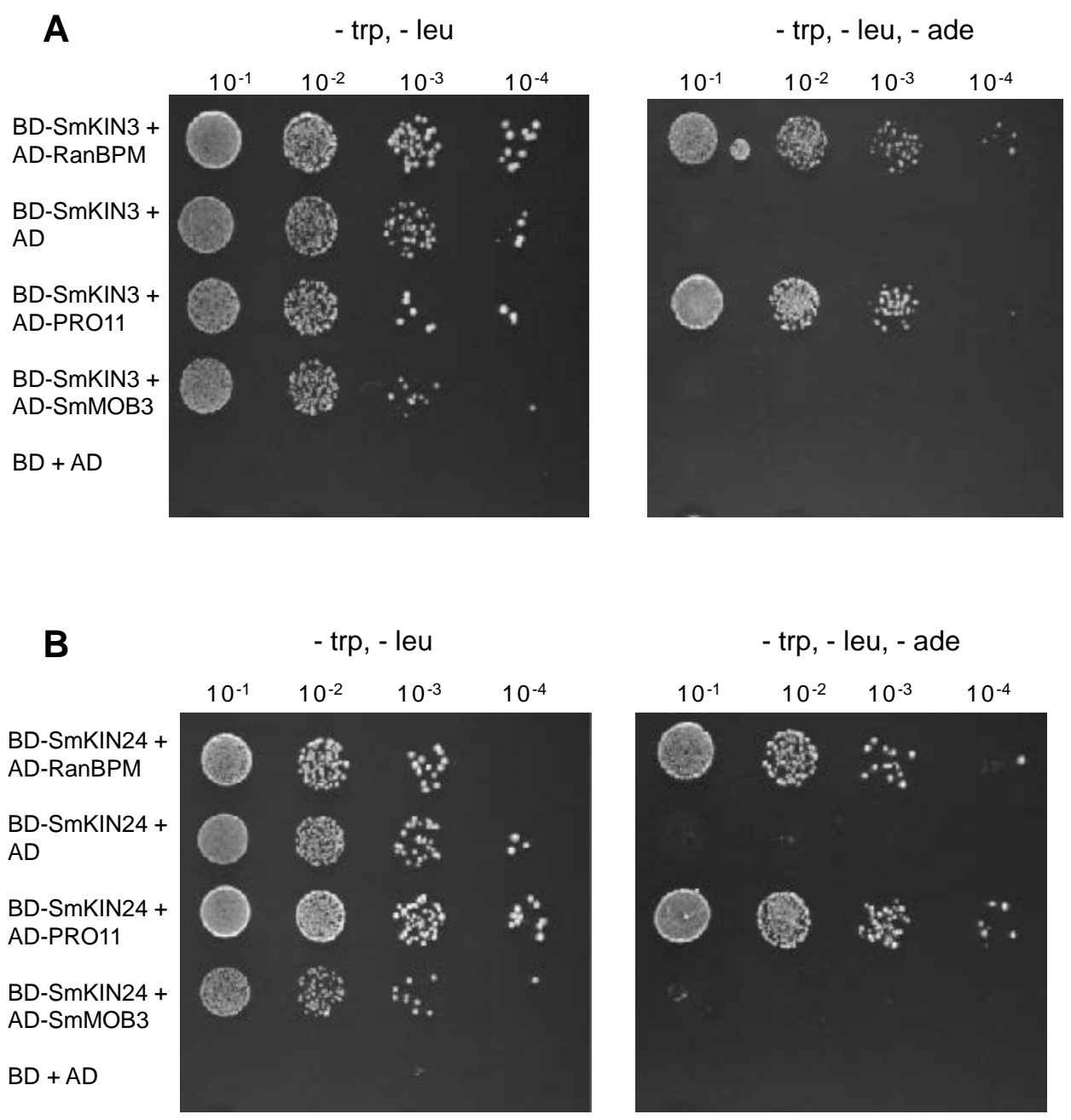

Figure 39. SmKIN3 and SmKIN24 interact physically with PRO11. Yeast two-hybrid analysis of interaction between SmKIN3 and PRO11 (A) as well as SmKIN24 and PRO11(B). For Y2H analysis, serial dilutions of diploids obtained after mating spread on medium lacking tryptophan (trp) and leucine (leu) or trp, leu and adenine (ade). BD = DNA-binding domain of GAL4, AD = activation domain of GAL4. GAL4 binding domain was fused to SmKIN3 (BD-SmKIN3) or SmKIN24 (BD-SmKIN24), GAL4 activation domain was fused to PRO11 full-length (AD-PRO11). A diploid strain carrying empty pGADT7 (AD) and pGBKT7 (BD) served as negative control. Expression of the GAL4-BD fusion protein from pBD-SmKIN3 (BD-SmKIN3) and pBD-SmKIN24 (BD-SmKIN24) plasmids were checked by mating Y187 transformants with AH109 carrying PAD-RanBPM (AD-RanBPM) (Tucker et al., 2009)

To test whether PRO11 and GCKs SmKIN3 and SmKIN24 could interact in vivo, we performed co-IP studies in S. macrospora. We expressed N-terminally FLAG-tagged SmKIN3 as well as SmKIN24 and HA-tagged PRO11 (Bloemendal et al., 2012) in S. macrospora. We were not able to express FLAG-tagged SmKIN24 in S. macrospora in amounts suitable for co-IP, under 
control of either the native or ccg1 promoter. All obtained viable transformants expressed Smkin24 in low amounts (data not shown). We therefore concluded that SmKIN24 is highly instable. However, tagged SmKIN3 and PRO11 could be expressed separately and co-expressed in wt S. macrospora. Pull-down experiments without adding a cross-linker did not result in precipitation of PRO11 (data not shown). Since protein kinases often interact weakly and transiently their substrate, we performed sulfhydryl cross-linking with bismaleimidohexane BHM which confirmed the physical interaction of full length SmKIN3 and PRO11 (Figure 40).

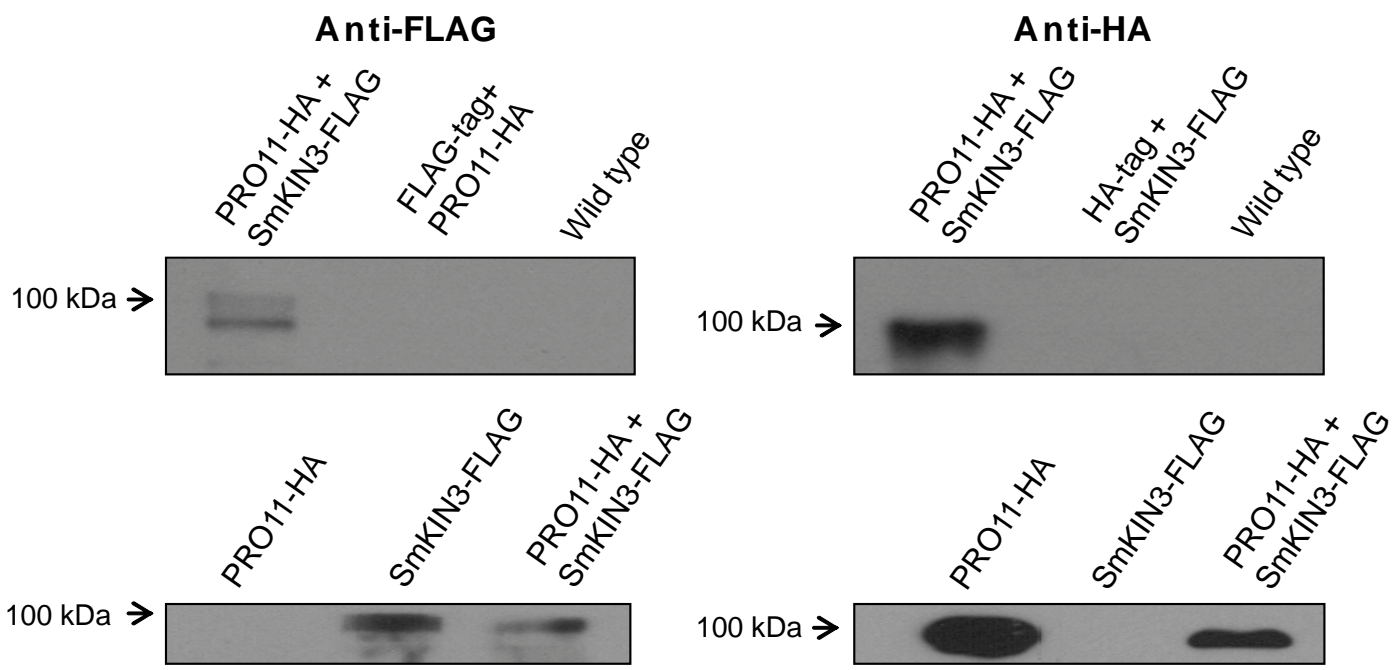

Figure 40. Co-IP of SmKIN3-FLAG and PRO11-HA. The Western-blot was performed with anti-FLAG and anti-HA antibody, respectively. Protein extract of the wt, separately expressed constructs and coexpression of each construct with complementary empty vectors served as controls.

\subsubsection{Deletion of Smkin3 or Smkin24 impairs vegetative growth but only $\Delta$ Smkin24 is sterile}

In order to investigate the functional role of Smkin3 and Smkin24, we generated single and double deletion strains $\Delta$ Smkin3, $\Delta$ Smkin24 and $\Delta$ Smkin3/ $\Delta$ Smkin24 (Figure 41 ). The respective genes were replaced by homologous recombination in the S. macrospora $\Delta \mathrm{ku} 70$ (Pöggeler \& Kück, 2006) strain (Figure 41A). The $\Delta$ ku70 background was removed afterwards by crossing the homokaryotic deletion strains with $S$. macrospora spore-color mutant r2 (Teichert et al., 2014) and the obtained single-spore isolates were verified by PCR (Figure 41B) and Southern blotting (Figure 41C). 
Results

A

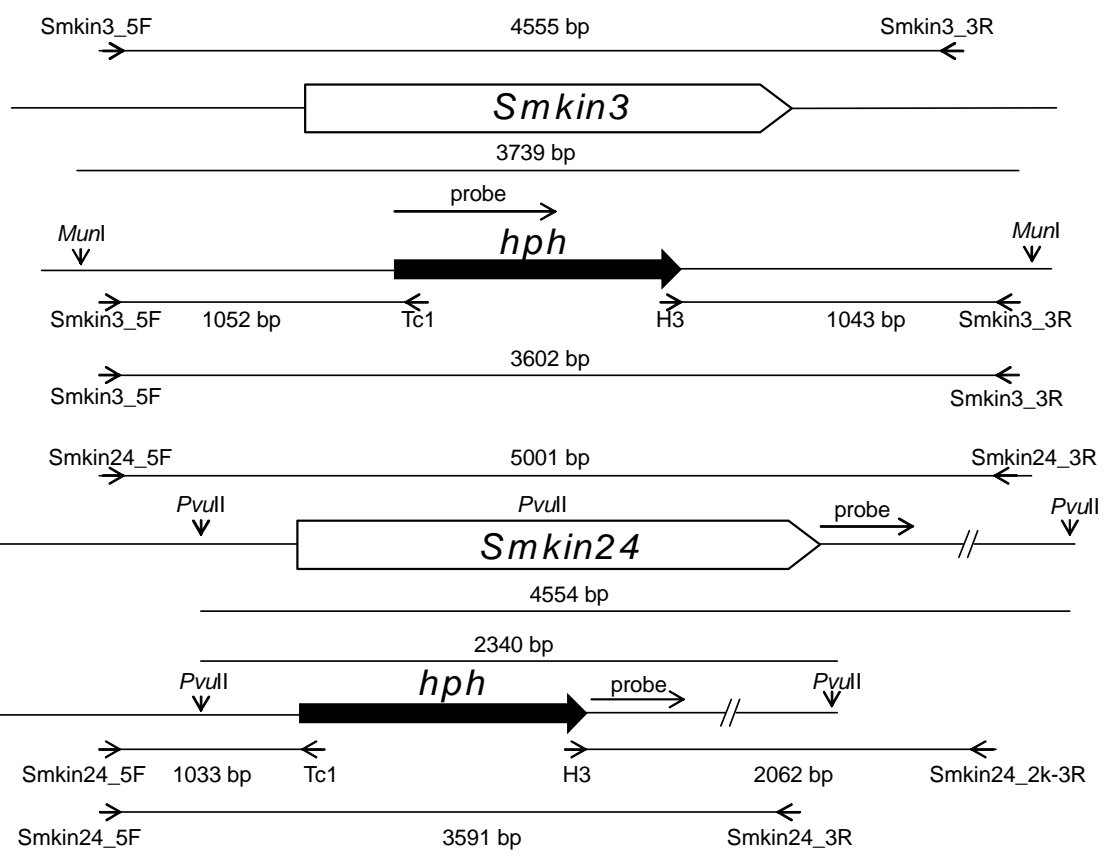

B

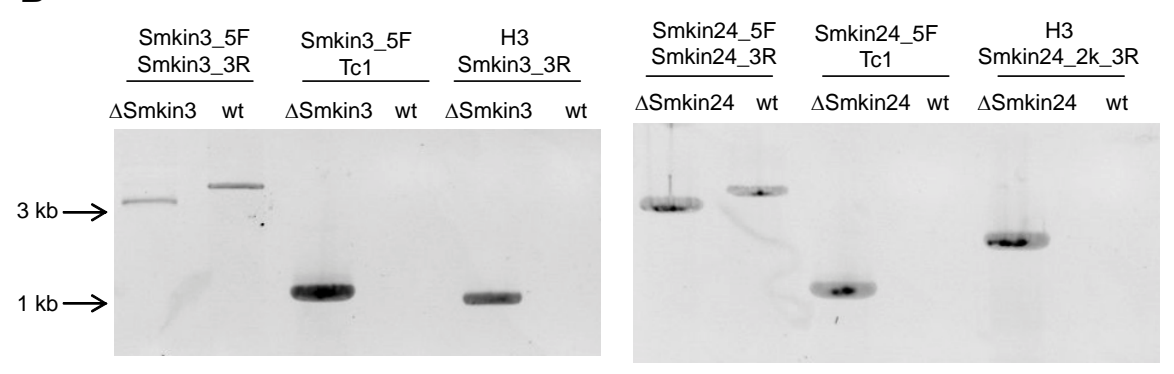

C

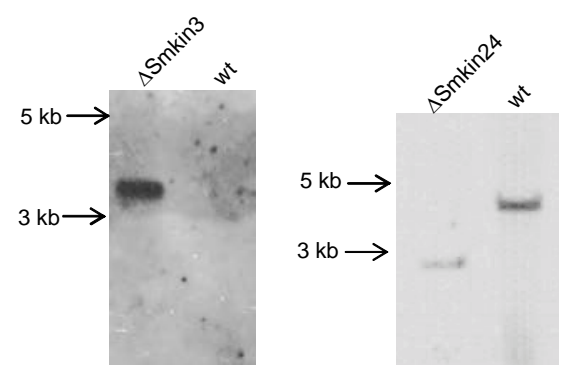

Figure 41. Generation of $\Delta$ Smkin3 and $\Delta$ Smkin24 deletion strains. (A) Schematic illustration of the Smkin3 and Smkin24 loci before and after homologous integration of the respective deletion cassette. Primers used for verification of the deletion strains are indicated by arrows. Sizes of PCR fragments and probes used for Southern hybridization are given. (B) Verification of the respective deletion using PCR. Sizes of amplicons and positions of the primers as indicated in (A). (C) Integration of the deletion cassette was verified by Southern hybridization (Sambrook et al., 2001). Positions of the respective probes are indicated in (A). $\Delta$ Smkin3 (left) was verified using a hygromycin specific probe that only binds within the deletion cassette. $\Delta$ Smkin24 (right) was verified using a probe binding at the 3' region of Smkin24. In this case, the successful integration is represented by a band shift. 
Microscopic analysis of the knockout strains revealed that only $\Delta$ Smkin3 was fertile and able to generate fruiting bodies with ascospores within 7 days (Figure 42).

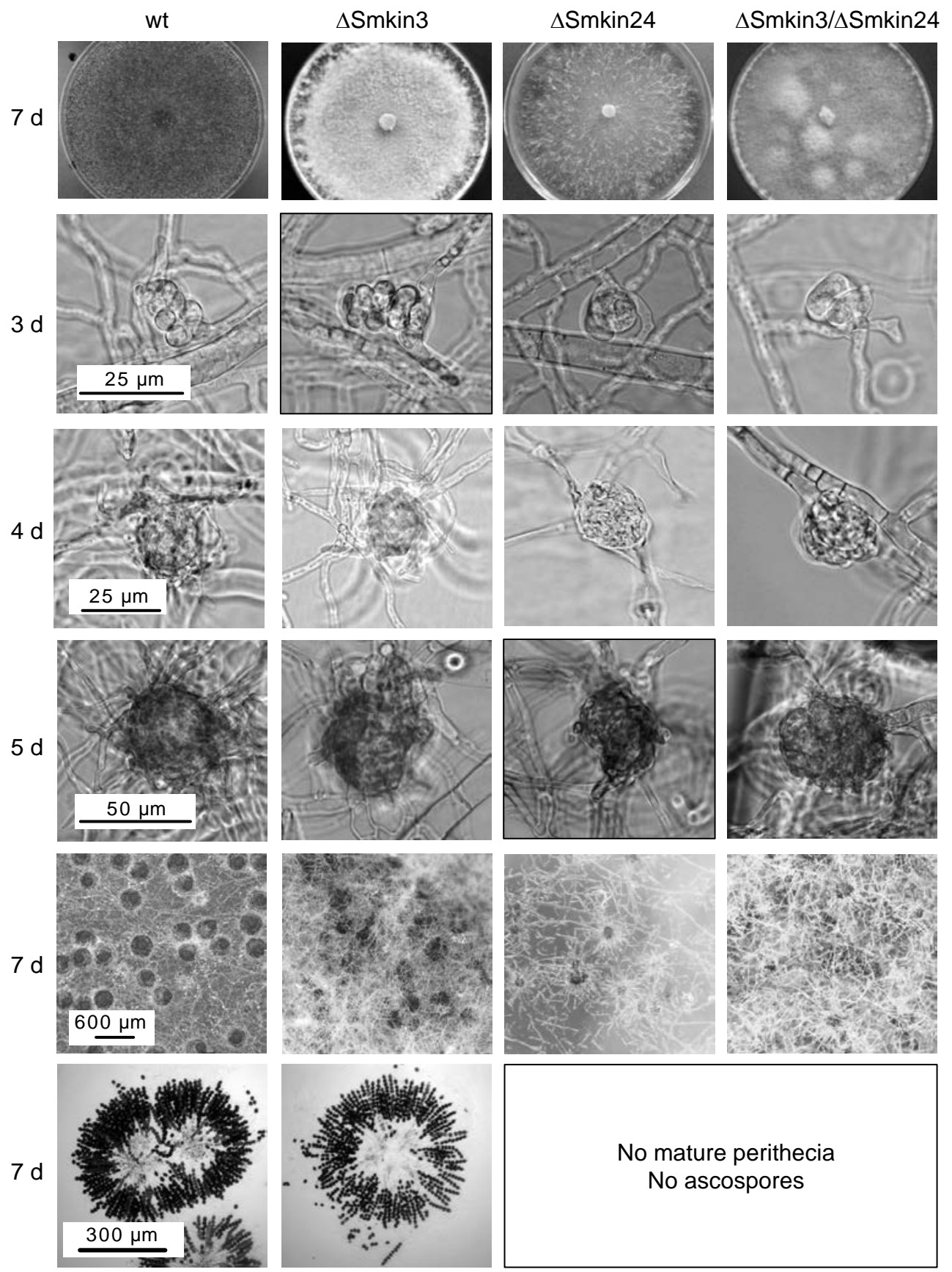

Figure 42. Macroscopic and microscopic analysis of the sexual development of wt, $\Delta$ Smkin $3, \Delta \operatorname{Smkin} 24$ and $\Delta$ Smkin3/ $\Delta$ Smkin24. The wt strain produces ascogonia after 3 days which develop to unpigmented protoperithecia at day 4 and pigmented protoperithecia at day 5. After 7 days mature perithecia with asci and ascospores are formed. Like the wt, $\Delta$ Smkin 3 completes the lifecycle within 7 days and produces germinable ascospores. Development of $\Delta$ Smkin24 and the double-deletion strain $\Delta$ Smkin $3 / \Delta$ Smkin 24 is arrested at the stage of late protoperithecia formation. Scale bars as indicated. 
The deletion strain $\Delta$ Smkin24 was halted during late protoperithecia maturation and thus failed to develop mature fruiting bodies (Figure 42). Additionally, $\Delta$ Smkin3 produced more aerial hyphae on solid SWG fructification medium compared to wt (Figure 42).

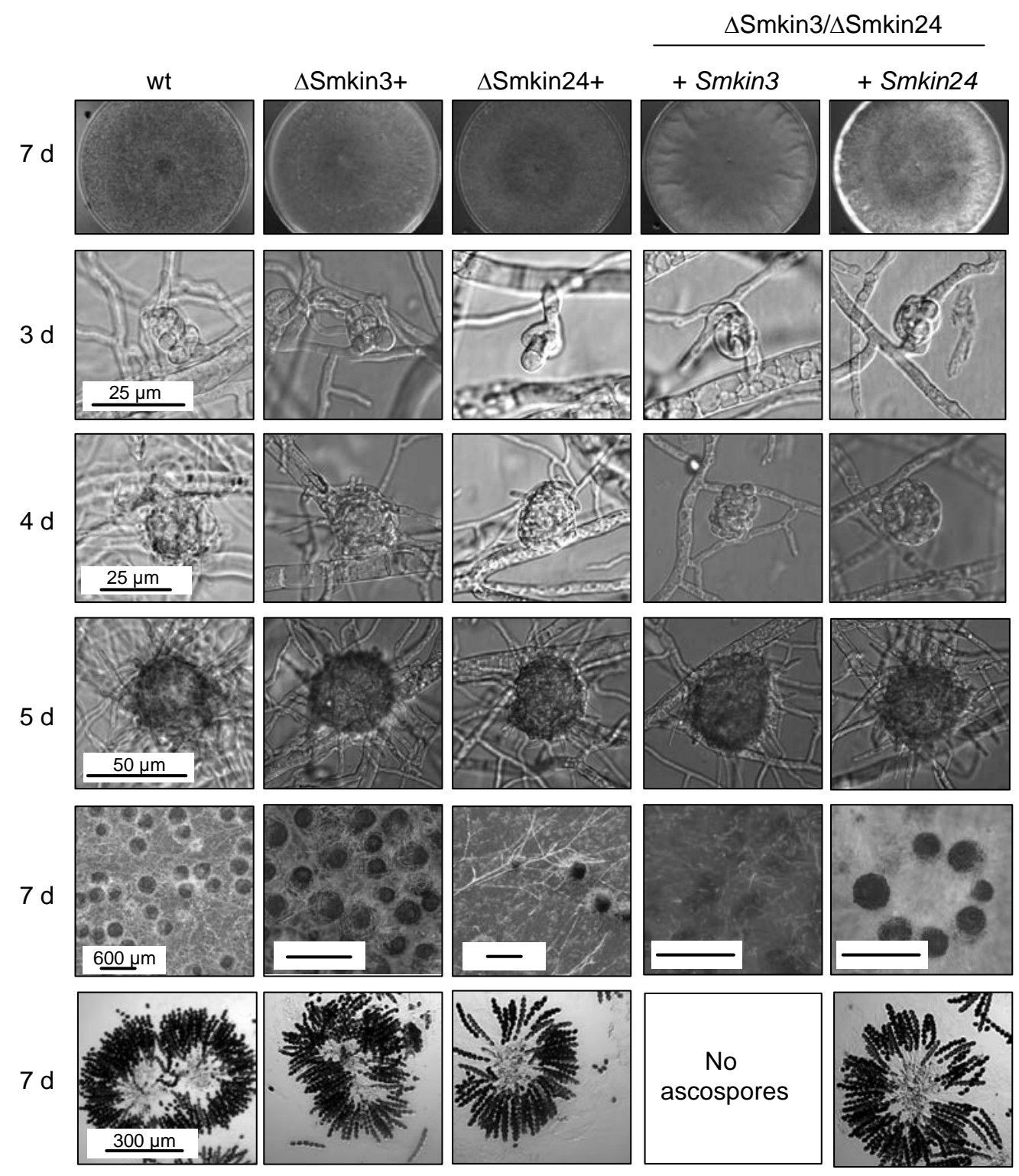

Figure 43. Macroscopic and microscopic analysis of the sexual development of wt, complemented $\Delta$ Smkin3 $(\Delta$ Smkin3 +$)$, complemented $\Delta$ Smkin24 $(\Delta$ Smkin24 +$)$ and partially complemented $\Delta$ Smkin3/ $\Delta$ Smkin24. The wt strain produces ascogonia after 3 days which develop to unpigmented protoperithecia at day 4 and pigmented protoperithecia at day 5. After 7 days mature perithecia with asci and ascospores are formed. Similar to the wt $\Delta$ Smkin $3+$ and $\Delta$ Smkin24+ and $\Delta$ Smkin $3 / \Delta$ Smkin $24+$ Smkin24 completed the lifecycle within 7 days and produced mature ascospores. Development of $\Delta$ Smkin3/ $\Delta$ Smkin24 + Smkin3 is arrested at stage of late protoperithecia formation, similar to $\Delta$ Smkin 24 . Scale bars as indicated. 
The double-deletion strain was generated by crossing the single deletion strains, and $\Delta$ Smkin3/ $\Delta$ Smkin24 exhibited a phenotype that was a combination of the phenotypes of both single deletion strains (sterility and increased aerial hyphae on solid SWG medium). Each of the described phenotypes could be complemented by inserting a copy of the deleted gene ectopically as shown in Figure 43.

Sterility of STRIPAK mutants pro11, pro22 and $\Delta$ Smmob3 is accompanied by defects in hyphal fusion (Bernhards \& Pöggeler, 2011, Bloemendal et al., 2012). However, in contrast with these previously characterized mutants, fusion of vegetative hyphae was not affected in $\Delta$ Smkin 3 and $\Delta$ Smkin24 (Figure 44). Furthermore, deletion of Smkin3 and Smkin24 impaired vegetative growth of $S$. macrospora: Growth velocity experiments revealed that $\Delta$ Smkin3 $(1.70 \pm 0.24$ $\mathrm{cm} / \mathrm{d}), \Delta$ Smkin24 $(1.82 \pm 0.41 \mathrm{~cm} / \mathrm{d})$ and $\Delta$ Smkin3/ $\Delta$ Smkin24 $(1.54 \pm 0.28 \mathrm{~cm} / \mathrm{d})$ grew more slowly than wt $(3.09 \pm 0.37 \mathrm{~cm} / \mathrm{d})$ (Figure 44).
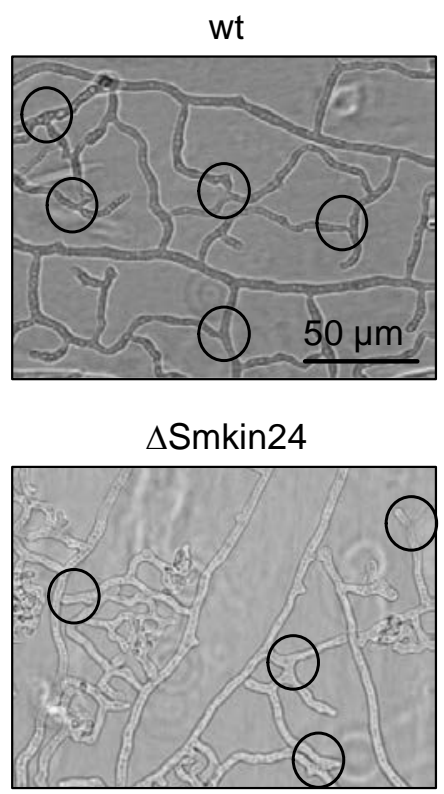

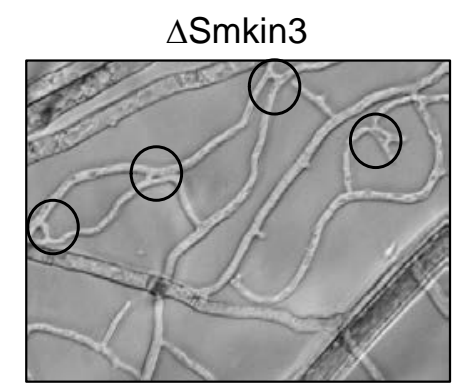

$\Delta$ Smkin3/ $\Delta$ Smkin24

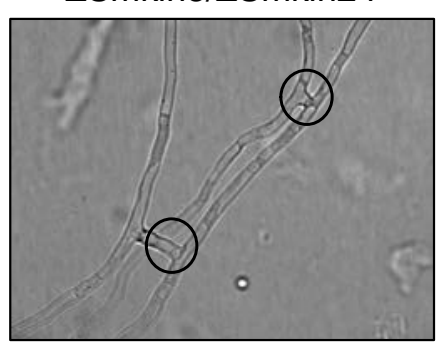

Figure 44. Microscopic investigation of hyphal fusion in wt, $\Delta$ Smkin3, $\Delta$ Smkin24 and

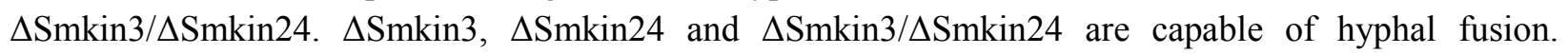
Hyphal fusion events are highlighted with circles. Pictures of hyphal fusion events were taken at subperiphal regions $10 \mathrm{~mm}$ behind the growth front. Hyphal-fusion was investigated 2-3 days past inoculation.

Furthermore, the deletion of Smkin3 and Smkin24 impairs vegetative growth of S. macrospora. Measuring growth velocity in race tubes over ten days revealed that $\Delta$ Smkin3 $(1.70 \pm 0.24$ $\mathrm{cm} / \mathrm{d}), \Delta$ Smkin24 $(1.82 \pm 0.41 \mathrm{~cm} / \mathrm{d})$ and $\Delta$ Smkin3/ $\Delta$ Smkin24 $(1.54 \pm 0.28 \mathrm{~cm} / \mathrm{d})$ display a reduced growth velocity than wt $(3.09 \pm 0.37 \mathrm{~cm} / \mathrm{d})$. 


\subsubsection{SmKIN3 and SmKIN24 localize to septa and influence septum formation}

In order to determine the localization of SmKIN3 and SmKIN24 in vivo, fluorescence microscopy was performed. Genes coding N-terminally eGFP-tagged full-length SmKIN3 or SmKIN24 were expressed in the respective S. macrospora deletion strains, which show that both kinases were localized at the septa (Figure 45). However SmKIN3 was localized mainly at the outer part of the septum, whereas SmKIN24 was localized at the middle (Figure 45) and this was verified by co-staining with calcofluor white (Figure 45).

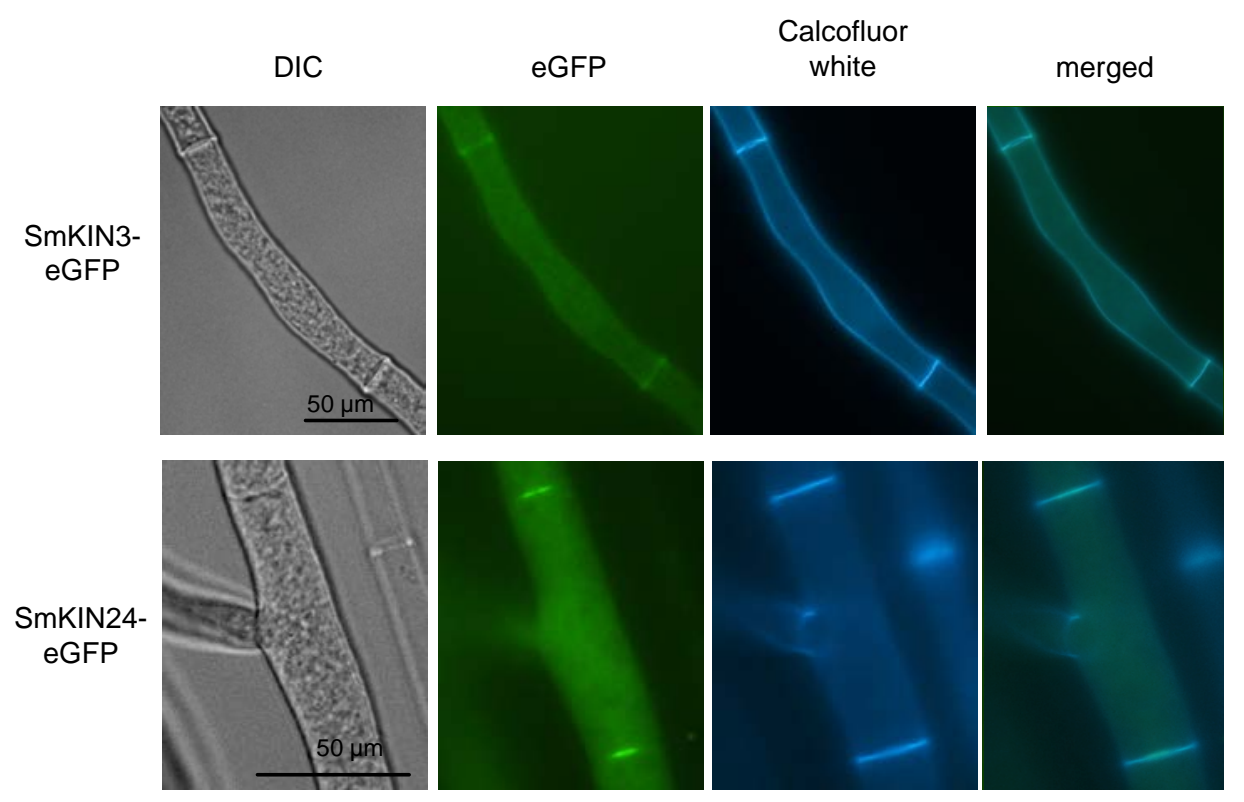

Figure 45. Localization of SmKIN3-eGFP and SmKIN24-eGFP in S. macrospora. SmKIN3-eGFP and SmKIN24-eGFP localize to septa. For visualization of cell walls and septa, hyphae were co-stained with calcofluor white. Scale bars as indicated.

These findings lead us to quantify septum formation in the $\Delta$ Smkin3, $\Delta$ Smkin2 4 and $\Delta$ Smkin3/ $\Delta$ Smkin24 strains by staining with calcofluor white after 18 or $32 \mathrm{~h}$ of growth (Figure 46). 


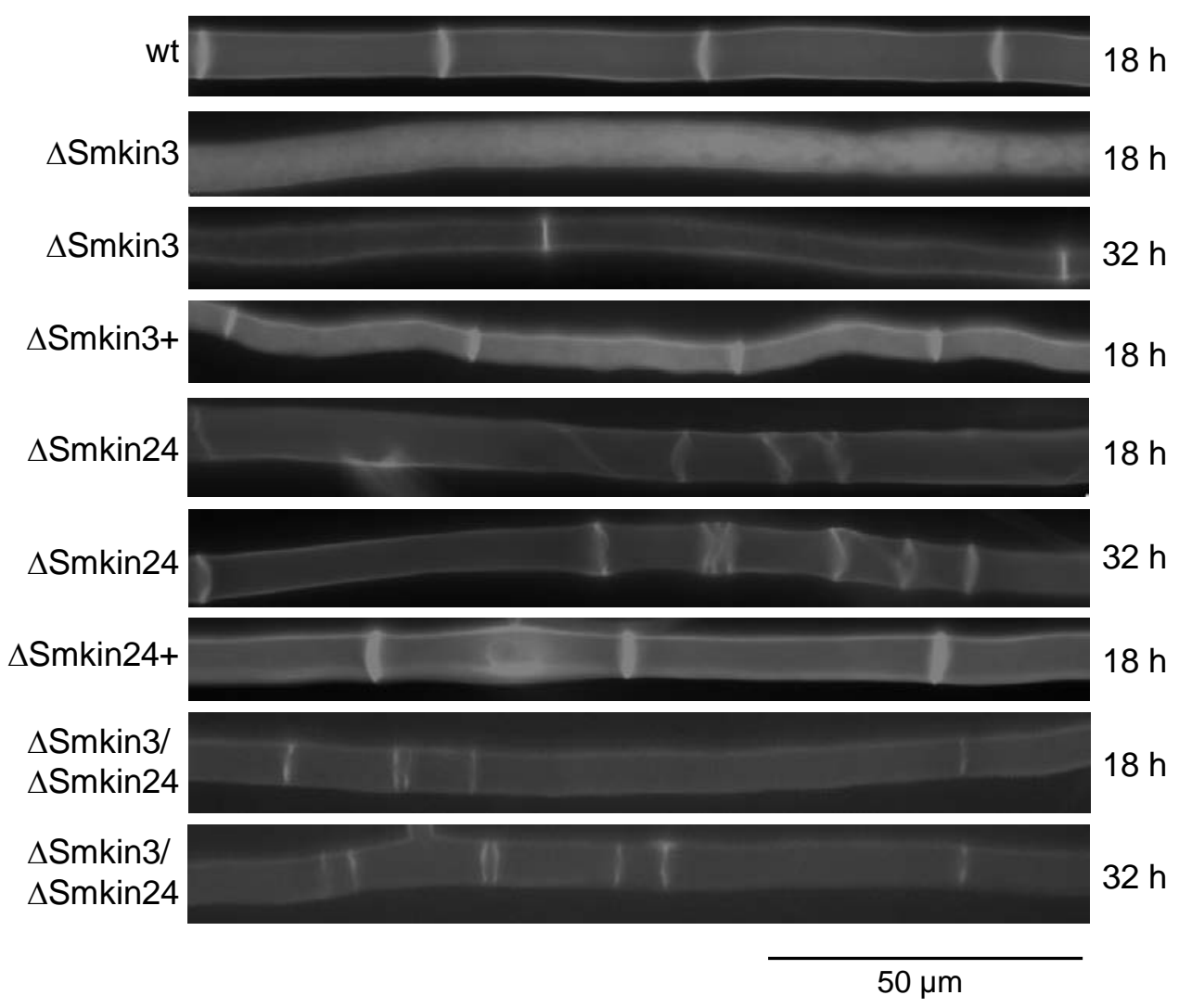

Figure 46. Analysis of septal development in wt, $\Delta$ Smkin $3, \Delta$ Smkin 24 and $\Delta$ Smkin $3 / \Delta$ Smkin 24 and complemented mutants. Distribution of septa was investigated under the microscope after $18 \mathrm{~h}$ and $32 \mathrm{~h}$ past inoculation. Septa were stained with calcofluor white.

Furthermore, we quantified the distances between adjacent septa in wt and deletion mutants after $24 \mathrm{~h}$ of growth (Figure 47). Septa were distributed in a uniform manner in wt with hyphal compartment length between 31-70 $\mu$ m (Figure 47). In contrast $\Delta$ Smkin3 exhibited larger distances between adjacent septa $(>71 \mu \mathrm{m})$, although this effect begun to revert after $24 \mathrm{~h}$ of growth (Figure 46). Strikingly, $\Delta$ Smkin24 developed numerous closely-packed septal bundles of abnormal shape, with much smaller hyphal compartments of 0-30 $\mu \mathrm{m}$ (Figure 47 ). $\Delta$ Smkin3 produced about half the total number of septa within a distance of $18 \mathrm{~mm}$ than wt, while $\Delta$ Smkin24 produced $20 \%$ more than wt after $24 \mathrm{~h}$ of growth. With respect to septation, the double deletion mutant $\Delta$ Smkin3/ $\Delta$ Smkin24 displayed a similar phenotype to the $\Delta$ Smkin24 single deletion strain. Ectopic integration of the respective wt gene into deletion mutants restored septal formation to wt level (Figure 46 and Figure 47). 


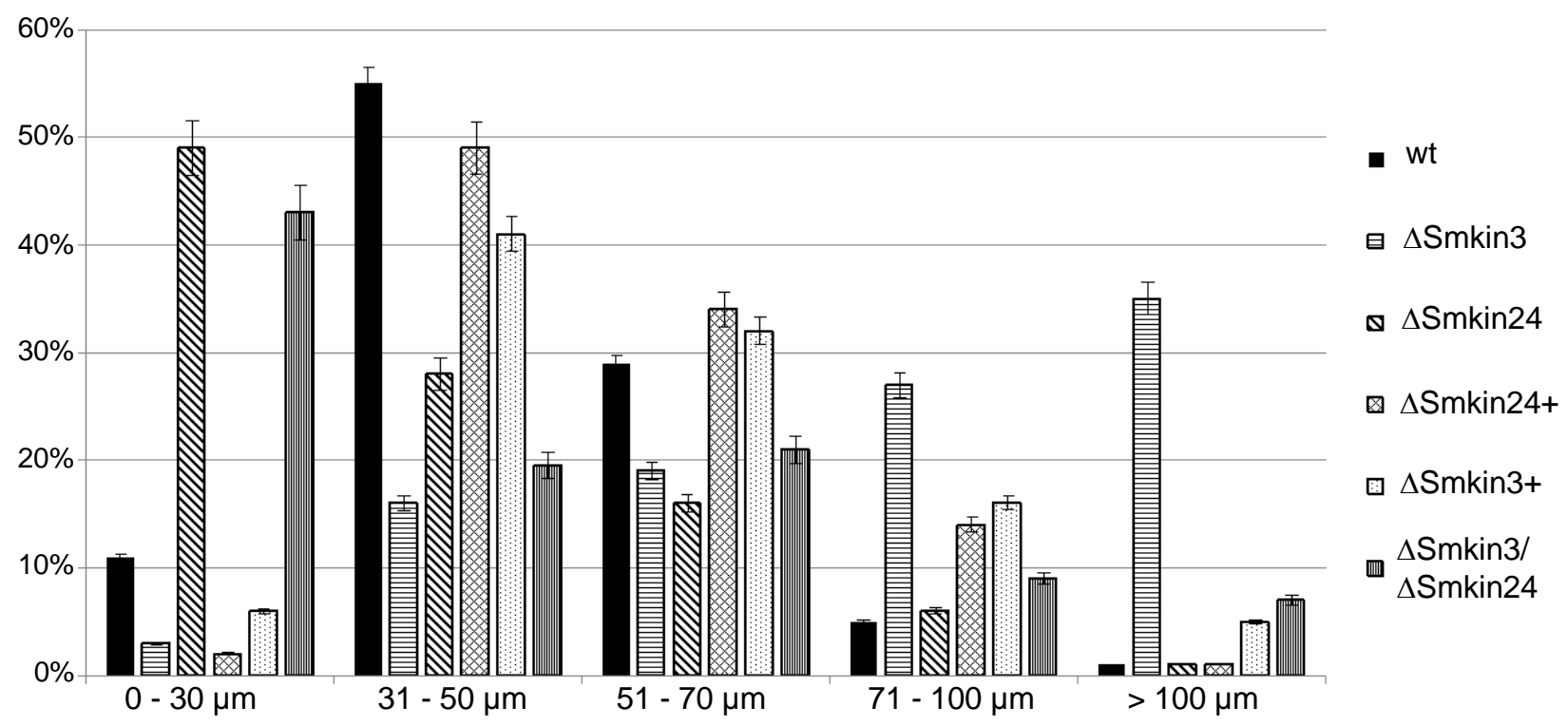

Figure 47. Quantitative analysis of septal development in wt, $\Delta$ Smkin3, $\Delta$ Smkin24 and $\Delta$ Smkin3/ $\Delta$ Smkin24 and complemented mutants. For quantification, distances between adjacent septa were measured over a distance of $18 \mathrm{~mm}$ per strain $24 \mathrm{~h}$ past inoculation. The total distance of $18 \mathrm{~mm}$ was divided into 30 segments of $600 \mu \mathrm{m}$. Measurements were binned into five different compartment lengths: $0-30 \mu \mathrm{m}, 31-50 \mu \mathrm{m}, 51-70,71-100 \mu \mathrm{mm}$, and more than $100 \mu \mathrm{m}$. The number of analysed compartments was normalized to $100 \%$. Error bars (SD) are given as indicated $(n=30)$. $\Delta$ Smkin $3+$, $\Delta$ Smkin24+ complemented mutants carrying an ectopic copy of the respective wt gene.

\subsection{5 $\Delta$ Smkin 3 protoplasts recover significantly faster than wt protoplasts}

We observed an increased growth rate in $\Delta$ Smkin3 protoplasts compared to wt, therefore we isolated protoplasts from wt, $\Delta$ Smkin3, $\Delta$ Smkin24 and $\Delta$ Smkin3 $/ \Delta$ Smkin24 strains using a previously described protocol (Nowrousian et al., 1999, Pöggeler et al., 1997) and adjusted the protoplast concentration to $4 \times 10^{4}$ protoplasts $/ \mathrm{ml}$. The protoplasts were spread on solid complete medium with 10.8\% saccharose (CMS) (Nowrousian et al., 1999) and microscopically and macroscopically analyzed after 24, 4 and 72 h of growth (Figure 48). After 24 h, wt protoplasts developed a slightly branched mycelium expanded further after 48 h (Figure 48). Mycelia were only faintly visible without magnification on agar plates after $72 \mathrm{~h}$. Regeneration of protoplasts from $\Delta$ Smkin24 resembled those of wt. In contrast, protoplasts from $\Delta$ Smkin3 and $\Delta$ Smkin3/ $\Delta$ Smkin24 recovered markedly faster within the first days than wt and mycelia generated by $\Delta$ Smkin3 protoplasts were much denser and clearly visible with the naked eye after $72 \mathrm{~h}$. Increased aerial hyphae were also present with $\Delta$ Smkin3 and this effect was complemented 
by ectopically integrated wt copy of Smkin3 in the $\Delta$ Smkin3 mutant (Figure 48). The accelerated growth rate of $\Delta$ Smkin3 vegetative mycelium disappeared after five days.

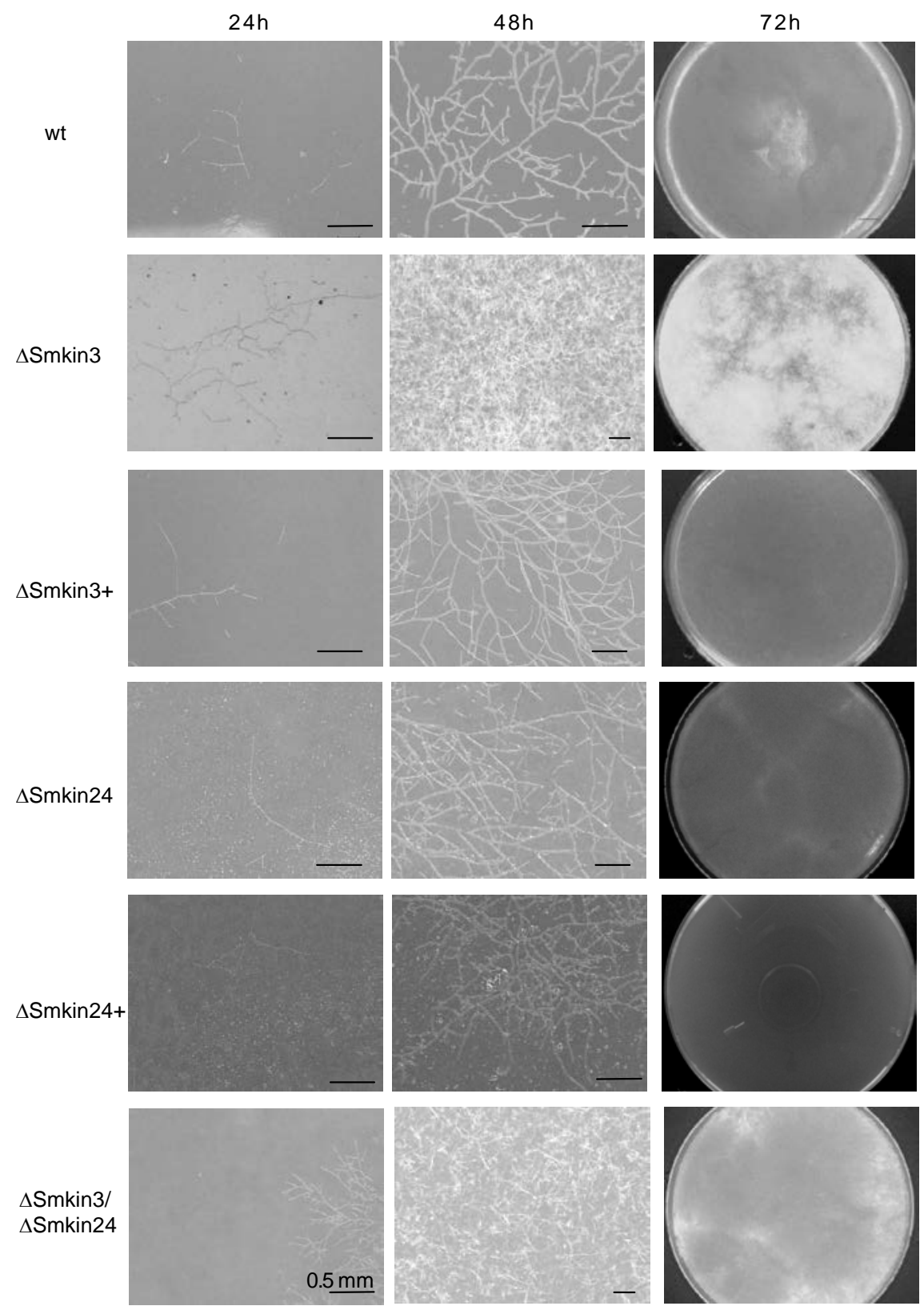

Figure 48. Investigation of protoplast recovery and vegetative growth of $\Delta \operatorname{Smkin} 3, \Delta \operatorname{Smkin} 24$ and $\Delta$ Smkin3/ $\Delta$ Smkin24. Protoplasts of the respective strains were obtained as described by Nowrousian $e t$ al. (1999) and spread on solid CMS agar plates. Microscopic pictures were taken after $24 \mathrm{~h}, 48 \mathrm{~h}$ past inoculation, pictures of agar plates $72 \mathrm{~h}$ past inoculation. Compared to wt, protoplasts obtained from $\Delta$ Smkin3 and $\Delta$ Smkin3/ $\Delta$ Smkin24 recovered and grew faster within the first 2-3 days. Recovery and vegetative growth of protoplasts obtained from $\Delta$ Smkin24 is equal to wt $\Delta$ Smkin3,$+ \Delta$ Smkin $24+$ complemented mutants carrying an ectopic copy of the respective wt gene. Scale bars as indicated. 


\section{Discussion}

In animals and fungi, STRIPAK and STRIPAK-like complexes are implicated in various cellular processes such as signaling, cell-cycle control, apoptosis, vesicular trafficking, Golgi assembly, cell polarity, cell migration and fusion, neural development and cardiac function (Hwang \& Pallas, 2014). In the filamentous ascomycete S. macrospora, the STRIPAK-like complex contains the Striatin homolog PRO11, the STRIP1/2 homolog PRO22, the SLMAP homolog PRO45, the phocein homolog SmMOB3 and structural and catalytic subunits of the protein phosphatase PP2A. Components of the STRIPAK complex are required for S. macrospora sexual differentiation and cell fusion (Bernhards \& Pöggeler, 2011, Bloemendal et al., 2012, Bloemendal et al., 2010, Pöggeler \& Kück, 2004, Nordzieke et al., 2014).

\subsection{The GPI-anchored protein SmGPI1}

\subsubsection{SmGPI1 is a GPI-anchored protein}

SmGPI1 was first discovered in a Y2H screen using SmMOB3 as bait. In silico analysis of SmGPI1 using SignalP (Petersen et al., 2011) and big-PI Predictor (Eisenhaber et al., 1998, Eisenhaber et al., 1999, Eisenhaber et al., 2000, Sunyaev et al., 1999) revealed a putative signal sequence and a region for GPI-anchor attachment (Figure 12). Posttranslational attachment of the GPI-glycolipid at the C-terminus of proteins generally results in anchoring to the plasma membrane outer leaflet (Fujita \& Kinoshita, 2012, Singh et al., 2011). The signal sequence is necessary, because synthesis and attachment of the GPI-anchor occur in the ER. Many proteins have been shown to contain a signal sequence for ER translocation, which generally consists of 5-30 aa at the N-terminus of proteins (Rapoport, 2007, Blobel \& Dobberstein, 1975). Signal sequences tend to form a single alpha-helix (Rapoport, 2007). Further in silico analysis with Quick_2D (Biegert et al., 2006) predicted a single alpha-helix for SmGPI1s putative signal sequence. Moreover, the putative signal sequence of SmGPI1 has been shown to be sufficient to transport eGFP to the ER and in case of an added ER-retention signal, to remain there. Thus, the signal sequence is functional (Figure 18). In contrast to proteins with other destinations after ER translocation, GPI-anchored proteins have a structured region for GPI-anchor attachment. 
Analysis of various GPI-anchored proteins revealed that the region for GPI-anchor attachment generally share identical features; it consists of an $\omega$-residue for GPI-anchor attachment, a polar spacer region between $\omega+3$ to $\omega+9$ and a hydrophobic tail from $\omega+10$ to the C-terminal end of the protein (Caro et al., 1997, De Groot et al., 2003, Mayor \& Riezman, 2004, Pierleoni et al., 2008). The presence of small side chain residues at position $\omega+1, \omega+2$ and $\omega+3$ is assumed to be necessary for transamidase cleavage (Pierleoni et al., 2008). The $\omega$-residue of SmGPI1 is predicted to be aa 228 , aa $\omega+1$ is arginine, $\omega+2$ is alanine and $\omega+3$ is serine; alanine and serine are small side-chain aa. However, aa $\omega^{+1}$ is arginine, which is a large aa. The polar spacer of SmGPI1 at position $\omega+3$ to $\omega+10$ has the aa sequence SSKRGFTG and thus, contains indeed with serine, threonine and glycine 50\% polar aa. Regarding the fact that two polar aa particularly enclose the polar region it is likely, that residue $\omega+10$ is also part of the polar spacer. Residues $\omega+11$ to $\omega+25$ represent with the sequence LLVAAVVVATVSGLL the hydrophobic tail. This aa sequence contains with leucine, valine and alanine about $87 \%$ hydrophobic aa (Figure 12). It was shown, that deletion of the region for GPI-anchor attachment of SmGPI1 impaired localization to the cell wall or membrane of the cell (Figure 16). Moreover, inspection of SmGPI1s aa sequence showed a sequence pattern similar to previously identified CFEM (common in several fungal extracellular membrane proteins) domains. These domains are primarily found in fungal GPI-anchored proteins bound to cell walls. CFEM domains are approximately 60 aa long and contain 8 spaced cysteine residues usually near the N-terminus of the respective protein (Vaknin et al., 2014, Kulkarni et al., 2003). SmGPI1 exhibits 14 cysteine residues, which are conserved among SmGPI1-orthologs in ascomycetes (Figure 13). Thus, it is likely, that these residues have a function similar to these of the CFEM domains. In addition, each predicted ortholog has a putative signal sequence and a putative region for GPI-anchor attachment, but these differ in their sequence. SmGPI1 is not homologous to the recently described N. crassa GPI-anchored protein HAM-7, which is involved in fruiting-body formation and hyphal fusion (Fu et al., 2011, Maddi et al., 2012).

\subsubsection{SmGPI1 is a dual targeted protein}

GPI-anchored proteins normally localize to the outer leaflet of the plasma membrane (Fujita \& Kinoshita, 2012, Singh et al., 2011). In S. cerevisiae, the region $\omega-1$ to $\omega-5$ can support membrane localization (Caro et al., 1997, Frieman \& Cormack, 2003, Hamada et al., 1999, 
Stefanova et al., 1991). Moreover, GPI-anchored proteins in ascomycetes can also be covalently bound to the cell wall (Gilbert et al., 2012). The switch between localization to the membrane or cell wall depends among other factors, on the presence or absence of dibasic residues at position $\omega-1$ or $\omega-2$ (Stefanova et al., 1991, Ouyang et al., 2013). Absence of dibasic residues at position $\omega-1$ and $\omega-2$ support localization of the anchored protein to the cell wall (Stefanova et al., 1991, Ouyang et al., 2013). Fluorescence microscopy and Western blot analysis of eGFP-tagged SmGPI1, however, showed localization at the plasma membrane or cell wall, a fluorescence pattern similar to mitochondrial localization and partial secretion (Figure 16, Figure 18 and Figure 19).

A high content of serine and threonine residues in GPI-anchored proteins was shown to support cell-wall attachment of GPI-anchored proteins, approximately $70 \%$ of S. cerevisiae cell-wall bound GPI-anchored proteins contain more than 30\% serine/threonine residues (Frieman \& Cormack, 2004).

Sequence analysis of SmGPI1 revealed a serine/threonine content of $20 \%$ in total, compared to the average in proteins of $7.6 \%$ for serine and $6 \%$ for threonine (Bruice, 2004), the serine content of SmGPI1 (12.7\%) but not the threonine (7.2\%) is significantly increased. These results indicate that the final destination of GPI-anchored proteins is mediated by several factors. For example, De Sampaio et al. (1999) showed for the glucanosyltransferase GAS1p in S. cerevisiae localization to the cell wall, although it contains a dibasic residue at position $\omega-1$ and $\omega-2$. SmGPI1-eGFP localizes to structures, resembling the plasma membrane or the cell wall (Figure 19). Moreover, after differential centrifugation, SmGPI1 was mainly found in the cell detritus, but in case of deletion of the predicted region for GPI-anchor attachment, it appears mainly in the cytosol (Figure 16). The cell detritus contained remnants after early centrifugation, such as parts of the cell wall. Thus, the region containing the $\omega$-residue might be crucial for localization of SmGPI1 to the outer leaflet of the plasma membrane. This data is also supported by eGFP localization of SmGPI1 without the GPI-anchor attachment region (Figure 19), which was no longer present at the cell wall or membrane (Figure 19). These findings are consistent with many studies, made with other organisms. Ouyang et al. (2013) showed recently that only the signal sequence and the region for GPI-anchor attachment comprising $\omega-10$ to the C-terminal end of cell-wall protein Mp1p, glucanosyltransferase Gel1 and Ecm33, which function in maintaining fungal cell-wall integrity and virulence, are sufficient for proper localization to the cell 


\section{Discussion}

membrane or the cell wall. In their experiments, they fused the respective signal sequences and omega regions to eGFP and thus, mediated eGFP localization to the cell wall and membrane. In contrast to De Sampaio et al. (1999), Ouyang et al. (2013) showed that mutation of the residues $\omega-1$ and $\omega$-2 can alter the protein localization. Cryptococcus neoformans chitin deacetylase 2 is an enzyme that converts chitin to chitosan and is an established virulence factor for C. neoformans infection. Gilbert et al. (2012) showed that the protein is bound to membranes and non-covalently associated to cell walls. Interestingly, cell wall association was independent from its GPI-anchor.

SmGPI1 full-length protein also appears in the cell-free medium (Figure 18). Deletion of its omega region not only impairs cell-wall localization, but slightly decreases secretion of the protein. Secretion of GPI-anchored proteins in general is well documented (Low, 1989, Mayor \& Riezman, 2004, Paulick \& Bertozzi, 2008). Djordjevic et al. (2005) showed that C. neoformans virulence factor phospholipase B1 localizes (Plb1) to the cell wall, membrane structures and was also detectable in the cell-free medium. Furthermore, that this localization pattern was GPIanchor dependent. Similar to SmGPI1, deletion of the GPI-anchor led to an increased secretion of the protein. Moreover, SmGPI1 was shown to localize to structures resembling mitochondria (Figure 19). This was confirmed by co-staining with MitoTracker Red and likely caused the signal detected for the membrane fraction after differential centrifugation (Figure 16). In eukaryotes, dual targeting of a single proteins to more than one subcellular compartment is well documented (Raza, 2011, Ben-Menachem et al., 2011, Dinur-Mills et al., 2008, Yogev et al., 2011). These examples include GPI-anchored proteins such as $S$. cerevisiae $\beta-1,3-$ glucanosyltransferase GAS1, which plays as a GPI-anchored cell-wall protein a role in the formation and maintenance of the cell wall and when targeted to the nucleus, in regulation of transcriptional gene silencing and rDNA stability. When targeted to the cell wall, GAS1 elongates and arranges 1,3-glucan side chains, which are linked to glucan, chitin and proteins, and in sum, form the main layer of cell walls. The sub population of GAS1 detected in the nucleus interacts with the histone deacetylase SIR2 and increases rDNA silencing in a SIR2dependent manner (Koch \& Pillus, 2009, Bauer et al., 2014). Pfeiffer et al. (2013) demonstrated that ER signal peptides of the GPI-anchored prion-like Shadoo, the neuropeptide hormone somatostatin, and the amyloid precursor protein mediate alternative targeting to mitochondria. This effect is mediated by structural features within the nascent chain; the signal sequences of 
each protein promotes proper ER import of the nascent chain containing alpha-helical domains, but unstructured polypeptides are targeted to mitochondria. Increased transport to mitochondria causes unproductive transport to the ER lumen, and vice versa. By this, they presented a novel mechanism of dual targeting of proteins to the ER or mitochondria, facilitated by structural features in the nascent chain.

In silico analysis using GlobPlot2 (Linding et al., 2003) revealed extended intrinsic disorder regions within SmGPI1 (Figure 49). The signal sequence of SmGPI1 seemed to be necessary for mitochondrial localization because N-terminally eGFP-tagged SmGPI1 (data not shown) and SmGPI1 with its first 27 aa deleted led to a diffuse localization to the cytosol. In contrast, removal of the C-terminal GPI-signal sequence slightly increased mitochondrial localization (Figure 19).

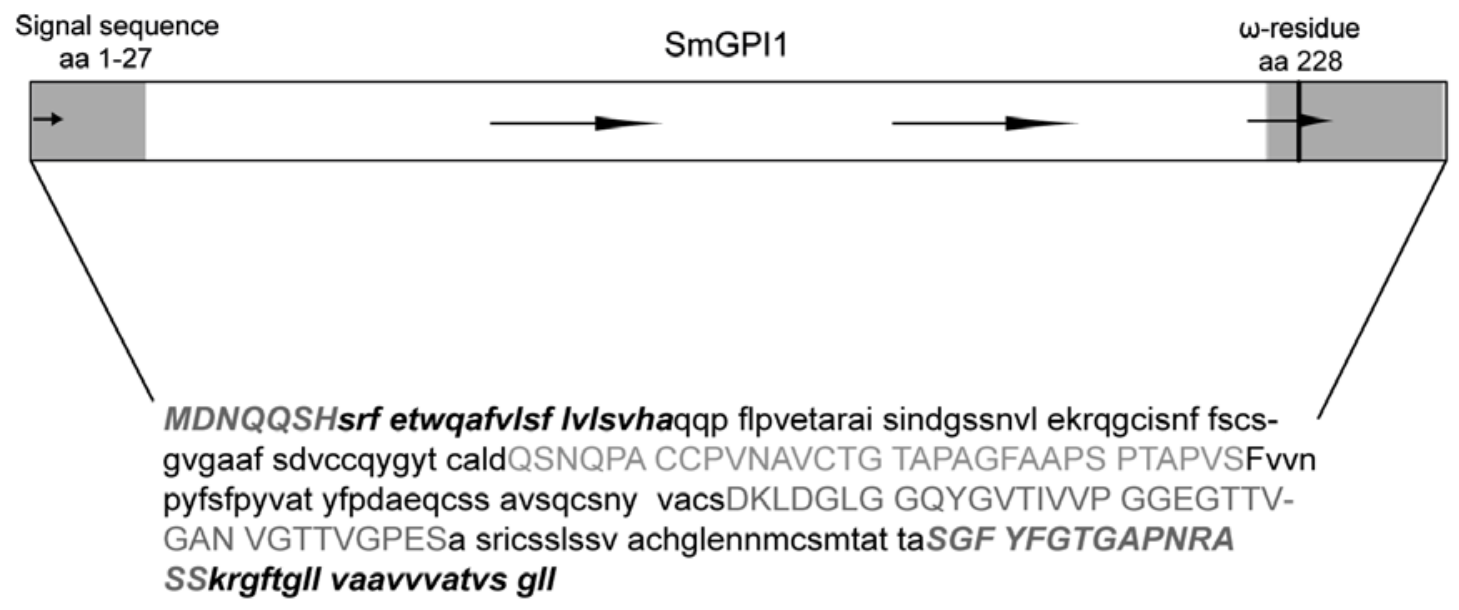

Figure 49. SmGPI1 exhibits regions of disorder. Shown is the protein precursor aa 1-253. The omega residue is marked in black. Grey boxes and italic letters in the aa sequence below represent N-terminal and C-terminal signal sequences. Arrows and grey capital letters in the aa sequence below represent disorder regions predicted by GlobPlot2 (Linding et al., 2003).

Similarly, Pfeiffer et al. (2013) showed that loss of the C-terminal GPI-signal sequence interferes with efficient ER import and increased mitochondrial import of mammalian GPI-anchored prion proteins. In this study was shown, that the C-terminal alpha-helical structure of Shadoo, which lies within the region for GPI-anchor attachment, can mediate ER import of the intrinsically disordered protein. In silico analysis with Quick_2D (Biegert et al., 2006) predicted for SmGPI1 
a single alpha-helical structure at the C-terminus. However, deletion of the C-terminal signal sequence of SmGPI1 did not completely impaired secretion but changed localization to the cell wall as shown by differential centrifugation and fluorescence microscopy (Figure 16 and Figure 19). The summarized mechanism of dual targeting of SmGPI1 in S. macrospora is shown in Figure 50.

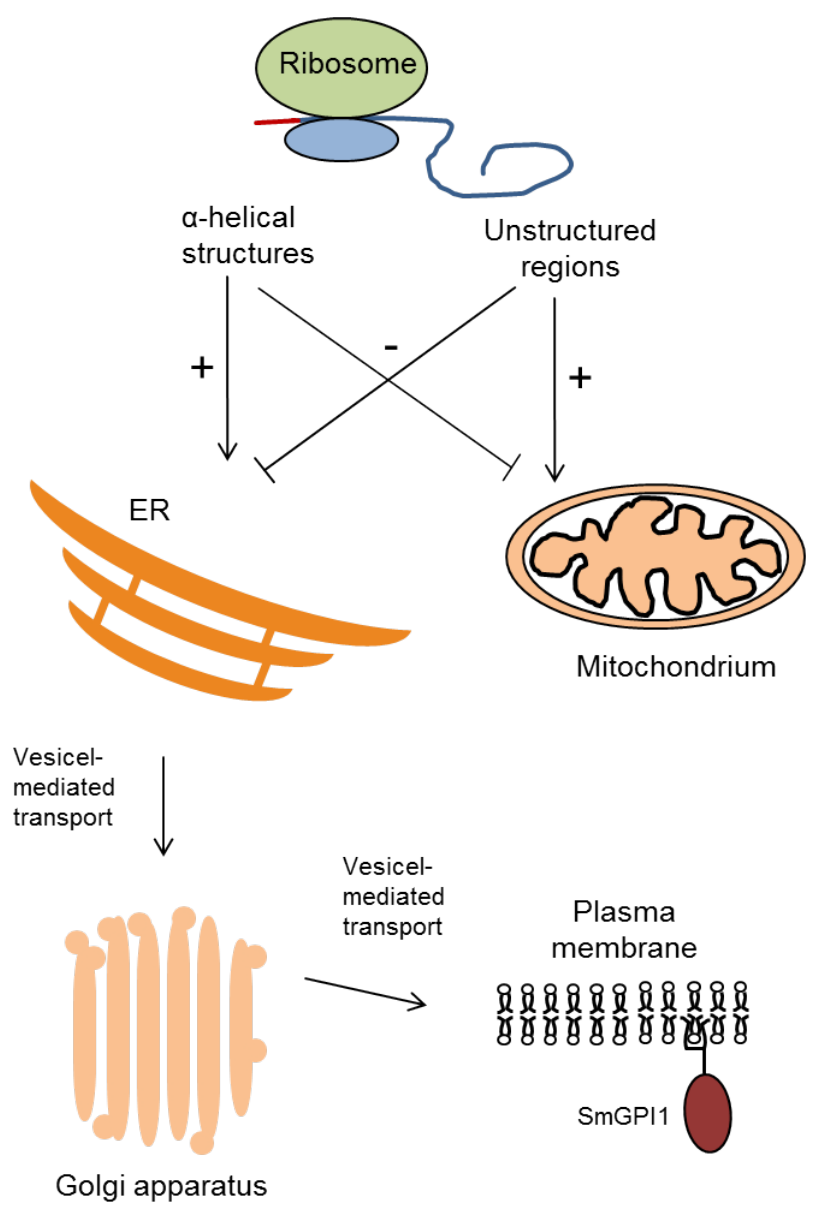

Figure 50. Dual targeting of SmGPI1 in S. macrospora. Features within the nascent chain determine the destination of SmGPI1, which is targeted to the cell wall and the mitochondria. Alpha-helical regions within the nascent chain facilitate transport to the ER and inhibit targeting to the mitochondria. Unstructured regions within the nascent chain regulate this process vice versa. For localization to the cell wall, the protein precursor is processed in the ER and transported via vesicles to the final destination. Based on the model presented by Pfeiffer et al. (2013). 


\subsubsection{STRIPAK protein SmMOB3 interacts physically with SmGPI1}

The GPI-anchored protein SmGPI1 was first identified as an interaction partner of SmMOB3 in a Y2H screen using a $N$. crassa cDNA library (Bernhards, 2010). This interaction was verified using S. macrospora cDNA and in a Y2H as well as by means of co-IP (Figure 14 and Figure 15), defined as a 144 aa N-terminal region of SmMOB3 and a 100 aa N-terminal region of SmGPI1. This region of SmMOB3 has recently shown to physically interact with the WD domain of Striatin homolog PRO11 in S. macrospora (Bloemendal et al., 2012). Since SmMOB3 was shown not to homodimerizes, it was suggested that the predicted alpha-helix of SmMOB3 mediates the SmMOB3-PRO11 interaction (Bernhards, 2010). Regarding that MOB3 interacts with several proteins via its $\mathrm{N}$-terminus, it is possible that interaction between SmMOB3 and PRO11 might alter SmMOB3-SmGPI1 binding and vice versa. This could be further investigated by co-IP analysis of SmMOB3-PRO11 interaction in strains with $\Delta$ Smgpil background compared to strains with wt background. Or, more appropriate, measuring binding kinetics of PRO11-SmMOB3 interaction in vitro before and after adding SmGPI1. Moreover, it would be interesting to analyze if these proteins exist in a tertiary complex, to gain a better understanding about the function of SmGPI1-SmMOB3 interaction.

As mentioned above, the final destination of fungal GPI-anchored proteins is the plasma membrane or the cell wall (Ouyang et al., 2013), but fungal MOB3/phocein homologs do not contain transmembrane domains (Maerz et al., 2009, Bernhards \& Pöggeler, 2011). Therefore, localization of SmGPI1 and SmMOB3 suggested that physical interaction of the proteins is prevented by their presence in different cell compartments. However, $N$. crassa MOB3 localizes at the nuclear envelope. This process requires HAM-2 (PRO22 homolog) and HAM-3 (PRO11 homolog) for membrane localization (Dettmann et al., 2013). Similarly, in S. macrospora, eGFPtagged SmMOB3 was detected at the nuclear envelope and in diffuse cytosolic patches (Figure 20).

Nordzieke et al. (2014) recently demonstrated that PRO45, a homolog of the mammalian STRIPAK core component SLMAP, interacts with PRO11 and SmMOB3 in S. macrospora. Moreover, PRO45 localizes at the mitochondria, showing that STRIPAK proteins in S. macrospora are mitochondria-associated (Nordzieke et al., 2014).

Mammalian $\mathrm{SG}_{2} \mathrm{NA}$ was detected in multiple cellular compartments including the plasma membrane, ER and mitochondria. $\mathrm{SG}_{2} \mathrm{NA}$ was shown to recruit protein kinase $\mathrm{B}$ (Akt) and the 
antioxidant protein DJ-1 into mitochondria and membranes (Tanti \& Goswami, 2014). Since SmMOB3 is a strong interaction partner of the S. macrospora Striatin homolog PRO11 (Bernhards \& Pöggeler, 2011, Bloemendal et al., 2012), one hypothesis is that PRO11 might mediate also SmMOB3 translocation to the mitochondria, where SmGPI1 was also shown to localize (Figure 19).

Under these conditions, portions of SmGPI1 and SmMOB3 share a common sub-cellular localization that allows direct physical interaction. This hypothesis could be further proved by isolation of mitochondria in strains, expressing tagged versions of SmGPI1 and SmMOB3 combined with Western blot analysis.

\subsubsection{SmGPI1 is a positive regulator of fruiting-body number}

In filamentous fungi, the development of multicellular fruiting bodies requires highly conserved differentiation processes and is essential for sexual reproduction (Pöggeler \& Kück, 2006). Various proteins function directly or indirectly in this process to determine the structure of the multicellular tissue of the fruiting bodies (Lord \& Read, 2011). In S. macrospora, deletion or mutation of genes encoding STRIPAK-associated proteins such as PRO11, PRO22, PRO45 or SmMOB3 cause sterility and thus, have a high impact on fruiting-body development (Bernhards \& Pöggeler, 2011, Bloemendal et al., 2012, Bloemendal et al., 2010, Nordzieke et al., 2014, Pöggeler \& Kück, 2004). Interestingly, all these mutants are capable of forming protoperithecia, suggesting that the main regulation of fruiting-body formation occurs after day three of development. In contrast, deletion of Smgpi1 did not lead to sterility, but increased the number of fruiting bodies although they were slightly reduced in size (Figure 30, Figure 31 and Figure 32). The deletion mutant completed the life cycle similar to wt after 7 days (Kück et al. 2009). This result suggested that SmGPI1 functions as a negative regulator of perithecia number and likely also as a positive regulator of perithecia size. Thus, SmGPI1 functions in this process but is not crucial for fertility and proper fruiting-body development. These results are confirmed by findings, that Smgpi1 overexpression decreased the number of perithecia per $\mathrm{cm}^{2}$ but these were slightly larger (Figure 32). Schindler \& Nowrousian (2014) previously reported about the S. macrospora polyketide synthase gene pks4. Deletion of the respective gene causes a developmental arrest at the stage of protoperethecia formation, but overexpression resulted in mount-like fruiting bodies, which kept growing even after 14 days, whereas perithecia growth in 
wt stops after 7 days. These mount-like fruiting bodies did not contain an increased number of ascospores, which is consistent with findings for $\Delta$ Smgpil. Although, the perithecia of $\Delta$ Smgpil were slightly smaller in this mutant, they contained equal numbers of spores compared to wt (Figure 31). In S. macrospora, energy and nutrients required to form multicellular fruiting bodies are at least partially supplied by the mycelium (Nowrousian et al., 1999, Voigt \& Pöggeler, 2013). The increased number of fruiting bodies of deletion mutant $\Delta$ Smgpi1 probably exhausted the mycelium and resulted in smaller perithecia. And referring to Smgpi1 overexpression that fewer fruiting bodies were supplied with more nutrients and grew slightly larger. Finally, based on these data a model for fruiting-body development in S. macrospora could be hypothesized. An initial input or signal leads to development of first sexual structures. After this step, a major checkpoint is reached, involving several gene products directly or indirectly by their function in signaling pathways. Afterwards, protoperithecia develop further to mature fruiting bodies. During the process of ripening, tissue-size control is mediated by proteins like PKS4 and SmGPI1. Moreover, only full-length version of the Smgpi1 could rescue perithecia number and size phenotypes, demonstrating that $\mathrm{N}$-terminal and $\mathrm{C}$-terminal signal sequences, and thus proper localization is required for SmGPI1 function in this process (Figure 30, Figure 31 and Figure $32)$.

\subsubsection{Smgpi1 deletion partially bypasses vegetative growth, hyphal fusion and fruiting- body development defects in $\Delta$ Smmob3}

Deletion of Smmob3 leads to sterility, reduced vegetative growth and defects in hyphal fusion in the filamentous ascomycete S. macrospora (Bernhards \& Pöggeler, 2011). This is consistent with other phenotypes of STRIPAK-deletion strains, such as $\Delta$ pro11, $\Delta$ pro22 and $\Delta$ pro45 (Bloemendal et al., 2010, Bloemendal et al., 2012, Nordzieke et al., 2014). In contrast to this, deletion of Smgpi1 does neither affect vegetative growth and hyphal fusion nor sexual development. (Figure 23). Interestingly, deletion of Smgpi1 in a sterile $\Delta$ Smmob3 deletion background partially reverted the defects in vegetative growth, hyphal fusion and fertility and thus acted as an intergenic suppressor of Smmob3 deletion. However, deletion of Smgpi1 does not restore fertility of S. macrospora STRIPAK mutants $\Delta$ pro11, $\Delta$ pro22 and $\Delta$ pro45 (Figure 25, Figure 26 and Figure 27). The gene pro11 encodes the central STRIPAK component Striatin; 
pro22 encodes the STRIP1/2 homolog, whereas PRO45 is homologous to STRIPAK component SLMAP. In N. crassa, PRO11 and PRO22 are essential for assembly and function of the STRIPAK complex at the nuclear envelope. MOB3 and HAM-4/PRO45 are only peripheral subunits of the complex (Dettmann et al., 2013). Thus, genetic interaction between Smgpi1 and Smmob3 is specific since even the deletion phenotype of a second peripheral subunit of the STRIPAK complex could not be bypassed by Smgpi1 deletion.

Thus, the impact of pro11 and pro22 deletion on developmental processes in S. macrospora occurs in many ways compared to SmMOB3. The summarized findings offer for SmMOB3SmGPI1 interplay the model that SmMOB3 amplifies an incoming signal and SmGPI1 negatively regulates the SmMOB3-dependent signal required for fruiting-body development (Figure 51). The signal is probably derived from the cell wall, the plasma membrane or mitochondria and is transmitted to the STRIPAK complex, which modulates it further. Direct interaction between intracellularly localized SmGPI1 and SmMOB3 might modulate the signal or SmGPI1 in the plasma membrane or cell wall compartment might interact with undefined transmembrane proteins that transduce the signal. Referred to the different phenotypes of $\Delta$ Smgpi1 and $\Delta$ Smmob3 single- and double-deletion strains, we assume the following scheme: in the $\Delta$ Smgpil mutant, the incoming signal is amplified, resulting in an increased number of fruiting bodies. In $\Delta$ Smmob3, the signal negatively regulated by SmGPI1 is too weak to activate the SmMOB3-depleted STRIPAK complex. In the double-deletion mutant, the amplified signal is sufficient to activate the SmMOB3-depleted STRIPAK complex and fruiting-bodies develop although fewer than in the wt, caused by absence of the negative regulator SmGPI1 (Figure 51). Similar to the above mentioned regulation, SmGPI1 might not only negatively regulate fruitingbody formation, but also hyphal fusion and vegetative growth. Thus, it seems that SmMOB3 and SmGPI1 are regulators of developmental processes in S. macrospora. To further elucidate this, we have to increase our knowledge about the genetic interplay of signaling pathways, which in sum, might function as major regulators of fundamental processes. The interesting question that remains is, why does deletion of Smgpi1 in a sterile $\Delta$ Smmob3 background reclaim aside of fruiting-body development, also hyphal fusion and vegetative growth? The model outlined in Figure 51 explains only the bypassed sterility. This is based on data, that SmGPI1 functions in regulation of perithecia size and probably also in their number. However, $\Delta$ Smgpil does not grow faster than wt or tend to form more hyphal fusions. For these processes, the outlined model 
seems not to be appropriate. One reason might be that proteins not involved in fruiting-body development function in this processes and down regulate hyphal fusion and vegetative growth to a level as present in wt.

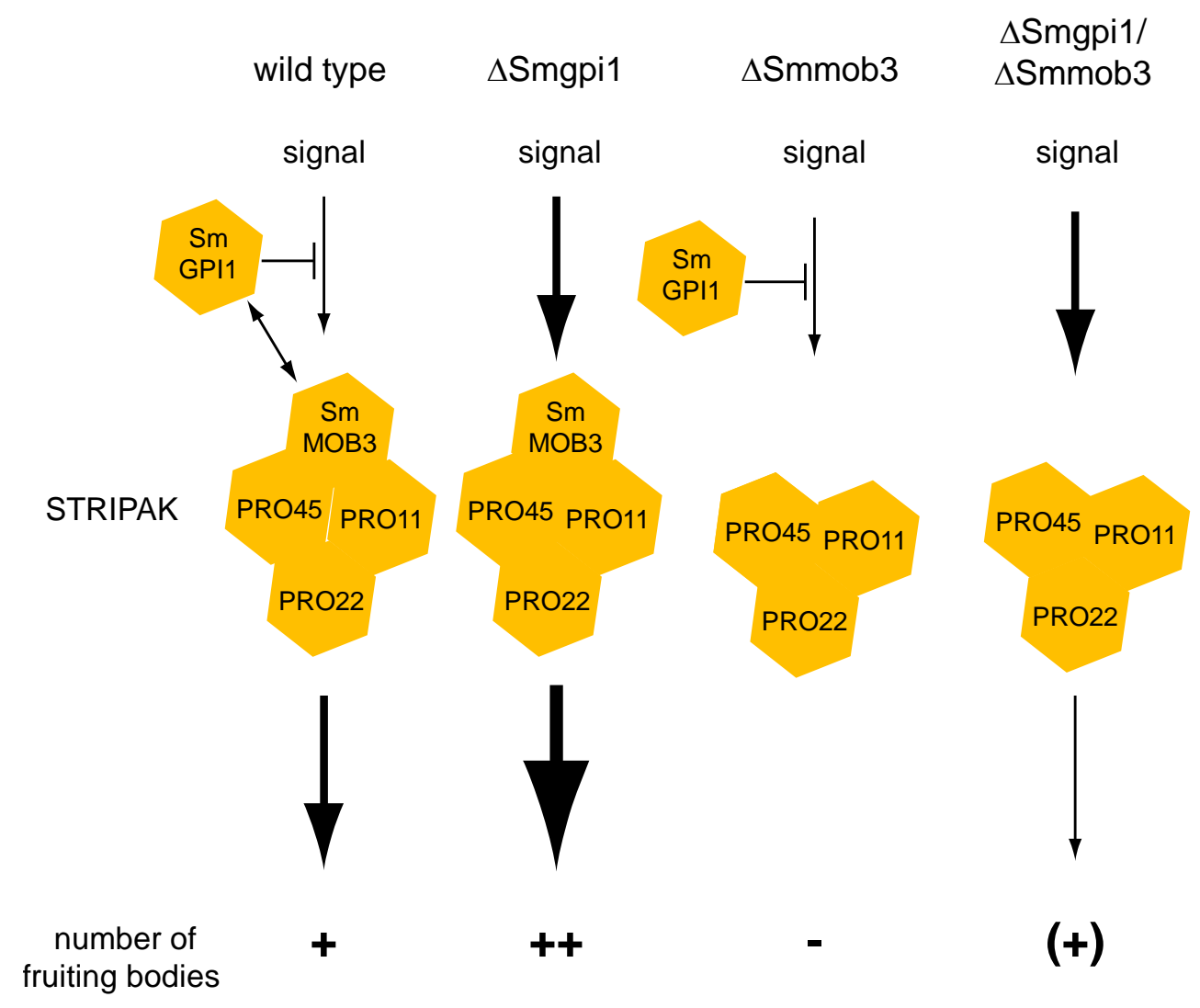

Figure 51. Schematic model of the genetic interplay between SmGPI1 and SmMOB3 and the STRIPAK complex in wt, single and double mutants. Double arrow indicates a direct physical interaction between SmGPI1 and SmMOB3. The thickness of arrows reflects the strength of the transmitted signal. Plus and minus symbols indicate the number of formed fruiting bodies

\subsection{The GCKs SmKIN3 and SmKIN24}

In mammals, the GCKs III MST4, STK24, STK25 and the GCK IV MINK1 are identified as components of the STRIPAK complex, but presence of STRIPAK-associated kinases has not yet been shown in filamentous fungi (1.3.1.4). To identify STRIPAK kinases in S. macrospora we performed a BLASTP search with the mammalian STRIPAK kinases MST4, STK24, STK25 and MINK1 against the S. macrospora genome. This identified SmKIN3 and SmKIN24 as putative homologs of mammalian STRIPAK-associated kinases. 


\subsubsection{Are SmKIN3 and SmKIN24 STRIPAK-associated kinases?}

The N-terminal part of the mammalian STRIPAK kinases is conserved among ascomycetes (Figure 33). GCKs have their enzymatic active region at the N-terminus, whereas the kinase domain of PAKs is located C-terminally (Dan et al., 2002, Delpire, 2009). Moreover, the phylogenetic allocation of MST4, STK24, STK25 and MINK1 with their orthologs in ascomycetes revealed two groups (SmKIN3-like and SmKIN24-like) of putative STRIPAKassociated GCKs in filamentous fungi (Figure 38). Thus, it seems that presence of these two GC kinases is conserved among ascomycetes. Interestingly, all identified putative homologs in yeast share the highest similarity with MINK1 except SID1 from S. pombe, which functions in the septation initiation network (SIN). The SIN is a signal transduction network required for proper coordination of mitosis and cytokinesis (Krapp \& Simanis, 2008). S. pombe SID1 seems to share slight similarity with the SmKIN3-like group, represented by its branching directly before the SmKIN3-like group (Figure 38).

We showed interaction of SmKIN3 and SmKIN24 with PRO11 in an Y2H system (Figure 39) and interaction of SmKIN3 and PRO11 by means of co-IP (Figure 40). However, successful coIP results required crosslinking and thus, indicate weak interaction. Since we were not able to overexpress Smkin24 properly, we assume this to be lethal as previously described for other proteins (Hein et al., 1997, Mahlert et al., 2006). In N. crassa, the GCK III NcPRK-9 (SID-1) (homolog of SmKIN3) is part of the fungal SIN, whereas NcSTK-6 (MST-1, homolog of SmKIN24, hereafter NcSTK-6) has a dual role in SIN and the morphogenesis Orb6 network (MOR). Following cytokinesis the morphogenesis network MOR is essential for cell-polarity control and septum formation (Kanai et al., 2005, Verde et al., 1998, Heilig et al., 2013, Heilig et al., 2014). The GCK III NcSID1 (hereafter NcPRK-9) has been shown to phosphorylate DFB-2 and thus trigger its activity during SIN formation (Heilig et al., 2013). The SmKIN24 homolog NcSTK-6 is needed for septal actomyosin ring formation and modulates MOR activity during septum formation in an antagonistic manner (Heilig et al., 2014).

NcPRK-9 and NcSTK-6 both localize to the septa and spindle-pole bodies (SPB) (Heilig et al., 2013, Heilig et al., 2014). In S. pombe, SIN components bind to a coiled-coil scaffold SID4 (Morrell et al., 2004). However, a clear homolog of the SIN scaffold SID4 has not been identified in ascomycetes so far (Heilig et al., 2013). Based on interaction studies, STRIPAK PRO11 may serve as scaffold for SmKIN3 and SmKIN24 and thus we hypothesized also 
interplay between STRIPAK and SIN in S. macrospora. This is supported by findings in fission yeast. SLMAP is a component of the mammalian STRIPAK complex (Goudreault et al., 2009) (1.3.1.7). The fission yeast SLMAP homolog CSC1p was shown to negatively regulate SIN and promotes SIN asymmetry (Singh et al., 2011). In addition, in D. melanogaster Ribeiro et al. (2010) showed that STRIPAK components negatively regulate HIPPO signaling, which is homologous to the fungal SIN network (Hergovich \& Hemmings, 2012). The HIPPO pathway is a central element of tissue-size control in D. melanogaster and higher organisms. It consists of an upstream kinase HPO (MST1/2 in mammals), the tumor suppressor mob family protein MATS and respective scaffolding units (Wei et al., 2007, Zhao et al., 2011). Based on interaction of HPO with homologs of MOB3 and Striatin, the D. melanogaster STRIPAK complex was identified as the negative regulator of HIPPO signaling and HPO was determined as homolog of mammalian STRIPAK kinases MST4, STK24 and STK25 (Ribeiro et al., 2010). A BLASTP analysis with HPO, MST1 and MST2 protein sequences revealed SmKIN3 and SmKIN24 as orthologs of these kinases in S. macrospora (data not shown). However, SmKIN3 and SmKIN24 did not interact with SmMOB3 in a Y2H (Figure 39). Recently, Dettmann et al. (2014) identified an interaction between NcSTK-6, the $N$. crassa homolog of SmKIN24, and components of the MAK-2 mitogen-activated protein kinases module (MAPKKK) NRC-1 and (MAPKK) MEK-2, involved in fungal communication between germinating spores (Dettmann et al., 2012, Fleissner et al., 2009). All three kinases of the MAK-2 MAPK module were shown to be weakly associated with STRIPAK components PP2A-A, PP2A-C, Striatin and MOB-3 in N. crassa (Dettmann et al., 2014). Thus, STRIPAK components and the SmKIN24 homolog function together in at least one cascade in $N$. crassa. In mammals, CCM3 recruits the GC kinases MST4, STK24 and STK25 but not MINK1 to Striatin (Goudreault et al., 2009, Gordon et al., 2011, Kean et al., 2011). However, a clear homolog of CCM3 has so far not been identified in ascomycetes. Thus, we assume that STRIPAK-associated kinases in S. macrospora are not recruited by other proteins to PRO11.

We showed that Smkin24 is alternatively spliced and has at least 3 isoforms (Figure 35). This is consistent with the mammalian MST4, which also has 3 identified isoforms (Greenman et al., 2007, Ota et al., 2004, Qian et al., 2001). The first three introns of Smkin24 are localized at the region coding for the kinase domain. These three introns are conserved among ascomycetes (Data not shown). Possibly, expression of different SmKIN24 variants might serve for modified 
substrate specificity as shown for other kinases (Wansink et al., 2003) and thus, could help to explain a crosstalk between SIN and STRIPAK. The hypothesized crosstalk between SIN and STRIPAK is shown in Figure 52. However, interaction between PRO11, SmKIN3 and SmKIN24 should be verified by other methods to state these GCK III are clearly STRIPAK-associated kinases.

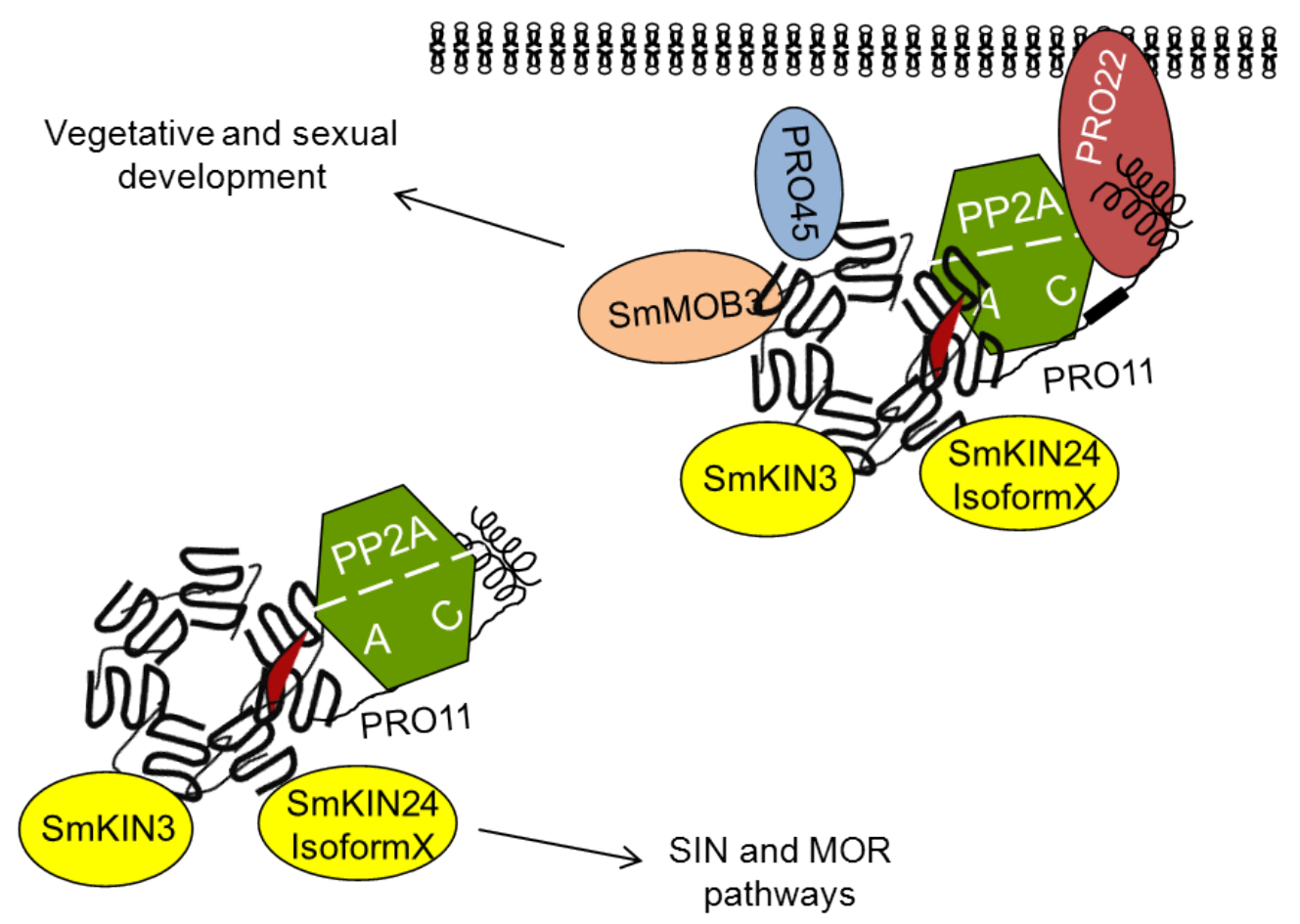

Figure 52. Schematic model for the interplay between STRIPAK and SIN in S. macrospora. SmKIN3 and SmKIN24 physically interact with PRO11, which functions as scaffold. Two subcomplexes are assumed, one containing PRO11, SmMOB3, PP2AA and PP2AC, PRO45, PRO22 and one or both kinases SmKIN3 and/or SmKIN24. This complex functions in vegetative and sexual development. The second complex contains PRO11, PP2AA and PP2AC and one or both kinases SmKIN3 and/or SmKIN24. This complex might function in SIN and MOR pathways.

The $N$. crassa homologs of SmKIN3 and SmKIN24 are components implicated in SIN and MOR pathways. Our data suggest an additional role of these GC kinases shown by a possible interaction with S. macrospora Striatin homolog PRO11. To close this knowledge gap, the interaction between PRO11 and SmKIN3 or SmKIN24 has to be tested by other in vivo methods, e.g. bimolecular-fluorescence complementation coupled with high-resolution microscopy, which could also give more information about the localization of a possible interaction. In addition, interactome studies that are capable to identify also short time interaction partners will help 
unravel how the crosstalk between signal pathways and their modulation by complexes is achieved.

\subsubsection{SmKIN3 and SmKIN24 affect growth velocity, sexual development and septum formation}

Deletion of Smkin3 does not impair fertility but leads to an increased formation of aerial hyphae and reduced growth velocity. In contrast, the deletion strain $\Delta$ Smkin24 is sterile and its lifecycle halted at later protoperithecia development and is also impaired in vegetative growth (Figure 42). A global phenotypic analysis of serine/threonine-protein kinase deletion mutants in $N$. crassa revealed that the $N$. crassa Smkin3 homolog prk-9 has an impact on asexual and sexual development as well as on vegetative growth and aerial hyphae formation (Park et al., 2011). S. macrospora undergoes only sexual development, so it is not possible to comment on asexual reproduction. We confirmed the results of Park et al. (2011) with respect to the reduced growth rate of the deletion mutant $\Delta$ Smkin3 but in contrast to their findings, deletion of Smkin3 did not impair fruiting-body development and fertility. Heilig et al. (2013) reported that $N$. crassa wt x $\Delta$ prk-9 crosses resulted in no abnormalities during sexual development. Deletion of stk-6 (homolog of Smkin24) in $N$. crassa resulted in reduced aerial hyphae and slightly reduced macroconidia production (Dvash et al., 2010). Similar to the S. macrospora $\Delta$ Smkin24 mutant which is halted at later protoperithecia development, in $N$. crassa $\Delta$ stk-6/Astk-6 crosses resulted in empty perithecia containing no ascospores. In contrast to the S. macrospora $\Delta$ Smkin24 the mycelial extension rates were not impaired in the $N$. crassa $\Delta$ stk6 mutant (Heilig et al., 2014). $N$. crassa mutant $\Delta$ prk-9 and $\Delta$ stk6 mutants showed hyphal tip swelling, bursting of hyphal tips followed by cytoplasmic leakage (Dvash et al., 2010, Heilig et al., 2013). This phenotype could not be observed in the $S$. macrospora $\Delta$ Smkin3 and $\Delta$ Smkin24 mutants. Similar to $N$. crassa, deletion of Smkin3 and Smkin24 causes defects in septa formation (Heilig et al., 2014, Heilig et al., 2013, Dvash et al., 2010). Consistent to the homologs in N. crassa, SmKIN3 and SmKIN24 localize to the septa (Figure 45), but could not be observed at spindle-pole bodies (Heilig et al., 2014, Heilig et al., 2013).

$\Delta$ Smkin3 strains exhibited less septa compared to wt and thus, formed elongated hyphal compartments (Figure 46 and Figure 47). This phenotype reverted, most probably due to the accumulation of suppressor mutations, after about 24 h of growth. This is similar to the $N$. crassa 
$\Delta$ prk-9 mutant producing initially aseptate germlings that formed septa at later stages of colony development (Heilig et al., 2013). In addition, germlings derived from $\Delta$ Smkin3 protoplasts grow faster than germlings of wt protoplasts (Figure 48). We hypothesized that nutrients, normally provided for septa formation, can be used for initial growth in the deletion strain and thus, may cause the increased initial growth velocity. $\Delta$ Smkin24 displays a contrary septation phenotype to $\Delta$ Smkin3; it generates more septa, partially gathered in bundle-like structures and abnormal in shape (Figure 46). Septal actomyosin tangel assembly, cortical actomyosin ring (CAR) assembly and CAR constriction are three consecutive stages of septum formation in $N$. crassa (Delgado-Alvarez et al., 2014). The septa observed in $\Delta$ Smkin24 resemble unfinished septa at early stages of septum formation, visualized by MYO-2 eGFP localization during SAT and CAR assembly in N. crassa (Delgado-Alvarez et al., 2014). Thus, SmKIN24 is required for proper CAR assembly as recently shown by Heilig et al. (2014) in $N$. crassa. Despite their contrary phenotypes, SmKIN3 and SmKIN24 function independently in S. macrospora. This was shown by the double-deletion mutant $\Delta \operatorname{Smkin} 3 / \Delta$ Smkin24, which exhibits the phenotypes of both single-deletion strains, however, with respect to septum formation it resembles the $\Delta$ Smkin24 mutant (Figure 46 and Figure 47).

\subsubsection{The STRIPAK (-like) complex in S. macrospora}

In filamentous fungi, development of multicellular fruiting bodies requires highly conserved differentiation processes and is essential for sexual reproduction (Pöggeler et al., 2006). For the ascomycete S. macrospora Bloemendal et al. (2012) recently showed that the STRIPAK complex, a multiprotein complex, functions in this process. The STRIPAK complex in mammals is known to be a major determinant in signaling (Figure 8). Deletion of genes, encoding for the STRIPAK-associated proteins PRO11 (Striatin homolog), PRO22 (STRIP1/2 homolog), PRO45 (SLMAP homolog) and SmMOB3 not only impair sexual development, but also hyphal fusion (Bernhards \& Pöggeler, 2011, Bloemendal et al., 2012, Bloemendal et al., 2010, Nordzieke et al., 2014). Thus, it seems that PRO11 in S. macrospora functions in developmental processes and serves in signaling concerning fruiting-body development and hyphal fusion. Today, evidence of STRIPAK-associated kinases in ascomycetes is still missing. This led to the name STRIPAK-like complexes, since presence of kinases was eponymous for the supramolecular complex (Frost et al., 2012, Goudreault et al., 2009). Based on interaction studies, we found 
indications for GC kinases linked to PRO11, making the STRIPAK-like complex to a full STRIPAK complex (Figure 39 and Figure 40). The following model is based on interaction and localization studies as well as gene characterization and presents todays knowledge combined with our data and indications about the STRIPAK complex in the filamentous fungus S. macrospora. Shown are several complexes found at the nuclear envelope, the ER, the Golgi or mitochondria. The displayed functions of the proteins are based on phenotypical analysis after gene deletion or characterized interaction partners, e.g. EPS15, which is implicated in vesicular trafficking.

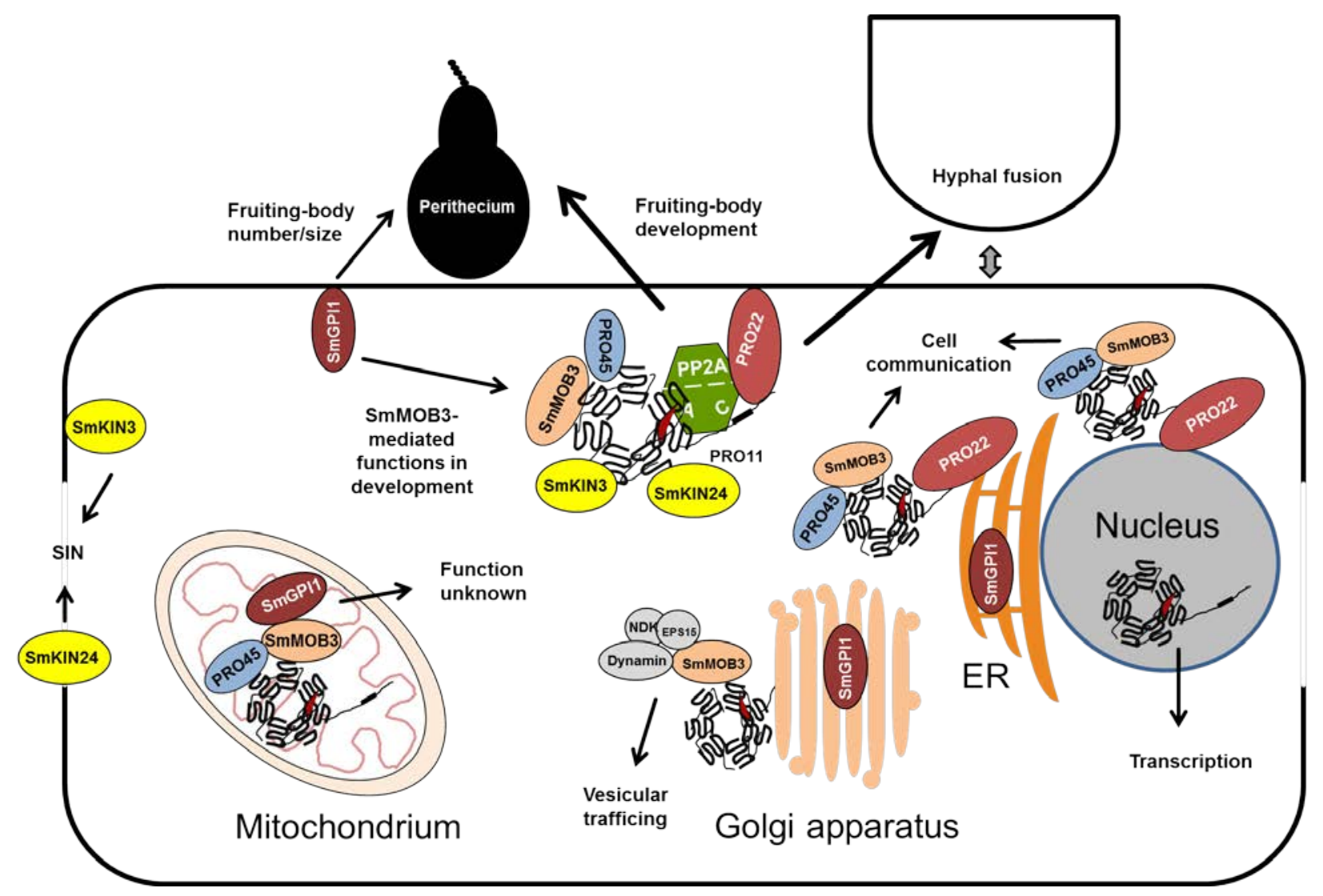

Figure 53. Schematic model of STRIPAK complex in S. macrospora. The multi-protein complex functions in hyphal fusion and fruiting-body development. It also might have a function in controlling the number and size of fruiting bodies as well as in vesicular trafficking. This model is based on the results gathered in this study and according to (Bernhards, 2010, Bernhards \& Pöggeler, 2011, Bloemendal et al., 2012, Nordzieke et al., 2014, Pöggeler \& Kück, 2004). 


\section{References}

Amberg, D., D. Burke \& J. Strathern, (2005) Methods in Yeast Genetics: A Cold Spring Harbor Laboratory Course Manual. Cold Spring Harbor Laboratory Press.

Andrade, M.A. \& P. Bork, (1995) HEAT repeats in the Huntington's disease protein. Nature genetics 11: 115-116.

Ariumi, Y., (2014) Multiple functions of DDX3 RNA helicase in gene regulation, tumorigenesis, and viral infection. Frontiers in genetics 5: 423.

Bai, S.W., M.T. Herrera-Abreu, J.L. Rohn, V. Racine, V. Tajadura, N. Suryavanshi, S. Bechtel, S. Wiemann, B. Baum \& A.J. Ridley, (2011) Identification and characterization of a set of conserved and new regulators of cytoskeletal organization, cell morphology and migration. BMC biology 9: 54 .

Baillat, G., S. Gaillard, F. Castets \& A. Monneron, (2002) Interactions of phocein with nucleosidediphosphate kinase, Eps15, and Dynamin I. The Journal of biological chemistry 277: 1896118966.

Baillat, G., A. Moqrich, F. Castets, A. Baude, Y. Bailly, A. Benmerah \& A. Monneron, (2001) Molecular cloning and characterization of phocein, a protein found from the Golgi complex to dendritic spines. Molecular biology of the cell 12: 663-673.

Bailly, Y.J. \& F. Castets, (2007) Phocein: A potential actor in vesicular trafficking at Purkinje cell dendritic spines. Cerebellum: 1-9.

Bartoli, M., A. Monneron \& D. Ladant, (1998) Interaction of calmodulin with striatin, a WD-repeat protein present in neuronal dendritic spines. The Journal of biological chemistry 273: 2224822253.

Bauer, H.C., I.A. Krizbai, H. Bauer \& A. Traweger, (2014) "You Shall Not Pass"-tight junctions of the blood brain barrier. Frontiers in neuroscience 8: 392.

Bechtel, S., H. Rosenfelder, A. Duda, C.P. Schmidt, U. Ernst, R. Wellenreuther, A. Mehrle, C. Schuster, A. Bahr, H. Blocker, D. Heubner, A. Hoerlein, G. Michel, H. Wedler, K. Kohrer, B. Ottenwalder, A. Poustka, S. Wiemann \& I. Schupp, (2007) The full-ORF clone resource of the German cDNA Consortium. BMC genomics 8: 399.

Becker, D.M. \& V. Lundblad, (2001) Introduction of DNA into yeast cells. Current protocols in molecular biology Chapter 13: Unit13.7.

Ben-Menachem, R., M. Tal, T. Shadur \& O. Pines, (2011) A third of the yeast mitochondrial proteome is dual localized: a question of evolution. PROTEOMICS 11: 4468-4476.

Bendixen, C., S. Gangloff \& R. Rothstein, (1994) A yeast mating-selection scheme for detection of protein-protein interactions. Nucleic acids research 22: 1778-1779.

Bendtsen, J.D., H. Nielsen, G. von Heijne \& S. Brunak, (2004) Improved prediction of signal peptides: SignalP 3.0. Journal of molecular biology 340: 783-795.

Benoist, M., A. Baude, A. Tasmadjian, B. Dargent, J.P. Kessler \& F. Castets, (2008) Distribution of zinedin in the rat brain. Journal of neurochemistry 106: 969-977.

Benoist, M., S. Gaillard \& F. Castets, (2006) The striatin family: a new signaling platform in dendritic spines. Journal of physiology, Paris 99: 146-153.

Bergametti, F., C. Denier, P. Labauge, M. Arnoult, S. Boetto, M. Clanet, P. Coubes, B. Echenne, R. Ibrahim, B. Irthum, G. Jacquet, M. Lonjon, J.J. Moreau, J.P. Neau, F. Parker, M. Tremoulet, E. Tournier-Lasserve \& N. Societe Francaise de, (2005) Mutations within the programmed cell death 10 gene cause cerebral cavernous malformations. American journal of human genetics $\mathbf{7 6}$ : 42-51. 
Bernhards, Y., (2010) Untersuchung der Fruchtkörperentwicklung bei dem Hyphenpilz Sordaria macrospora. In: Institute of microbiology and genetic. Georg-August-University Göttingen, pp. 125.

Bernhards, Y. \& S. Pöggeler, (2011) The phocein homologue SmMOB3 is essential for vegetative cell fusion and sexual development in the filamentous ascomycete Sordaria macrospora. Current genetics 57: 133-149.

Biegert, A., C. Mayer, M. Remmert, J. Soding \& A.N. Lupas, (2006) The MPI Bioinformatics Toolkit for protein sequence analysis. Nucleic acids research 34: W335-339.

Birnboim, H.C. \& J. Doly, (1979) A rapid alkaline extraction procedure for screening recombinant plasmid DNA. Nucleic acids research 7: 1513-1523.

Blobel, G. \& B. Dobberstein, (1975) Transfer of proteins across membranes. I. Presence of proteolytically processed and unprocessed nascent immunoglobulin light chains on membrane-bound ribosomes of murine myeloma. The Journal of cell biology 67: 835-851.

Bloemendal, S., Y. Bernhards, K. Bartho, A. Dettmann, O. Voigt, I. Teichert, S. Seiler, D.A. Wolters, S. Pöggeler \& U. Kück, (2012) A homologue of the human STRIPAK complex controls sexual development in fungi. Molecular microbiology 84: 310-323.

Bloemendal, S., K.M. Lord, C. Rech, B. Hoff, I. Engh, N.D. Read \& U. Kück, (2010) A mutant defective in sexual development produces aseptate ascogonia. Eukaryotic cell 9: 1856-1866.

Blondeau, C., S. Gaillard, J.P. Ternaux, A. Monneron \& A. Baude, (2003) Expression and distribution of phocein and members of the striatin family in neurones of rat peripheral ganglia. Histochemistry and cell biology 119: 131-138.

Bonangelino, C.J., E.M. Chavez \& J.S. Bonifacino, (2002) Genomic screen for vacuolar protein sorting genes in Saccharomyces cerevisiae. Molecular biology of the cell 13: 2486-2501.

Boyce, K.J. \& A. Andrianopoulos, (2011) Ste20-related kinases: effectors of signaling and morphogenesis in fungi. Trends in Microbiology 19: 400-410.

Breitman, M., A. Zilberberg, M. Caspi \& R. Rosin-Arbesfeld, (2008) The armadillo repeat domain of the APC tumor suppressor protein interacts with Striatin family members. Biochimica et biophysica acta 1783: 1792-1802.

Bruice, P.Y., (2004) Organic Chemistry. Pearson Education Inc.

Burkhard, P., J. Stetefeld \& S.V. Strelkov, (2001) Coiled coils: a highly versatile protein folding motif. Trends in cell biology 11: 82-88.

Burkhardt, J.K., D. Schmidt, R. Schoenauer, C. Brokopp, I. Agarkova, O. Bozinov, H. Bertalanffy \& S.P. Hoerstrup, (2010) Upregulation of transmembrane endothelial junction proteins in human cerebral cavernous malformations. Neurosurgical focus 29: E3.

Byers, J.T., R.M. Guzzo, M. Salih \& B.S. Tuana, (2009) Hydrophobic profiles of the tail anchors in SLMAP dictate subcellular targeting. BMC cell biology 10: 48.

Cardenas, W.B., (2010) Evasion of the interferon-mediated antiviral response by filoviruses. Viruses 2: 262-282.

Caro, L.H., H. Tettelin, J.H. Vossen, A.F. Ram, H. van den Ende \& F.M. Klis, (1997) In silicio identification of glycosyl-phosphatidylinositol-anchored plasma-membrane and cell wall proteins of Saccharomyces cerevisiae. Yeast 13: 1477-1489.

Carroll, A.M., J.A. Sweigard \& B. Valent, (1994) Improved vectors for selecting resistance to hygromycin. Fungal Genet Newslett 41: 22.

Castets, F., M. Bartoli, J.V. Barnier, G. Baillat, P. Salin, A. Moqrich, J.P. Bourgeois, F. Denizot, G. Rougon, G. Calothy \& A. Monneron, (1996) A novel calmodulin-binding protein, belonging to the WDrepeat family, is localized in dendrites of a subset of CNS neurons. The Journal of cell biology 134: 1051-1062. 
Castets, F., T. Rakitina, S. Gaillard, A. Moqrich, M.G. Mattei \& A. Monneron, (2000) Zinedin, SG2NA, and striatin are calmodulin-binding, WD repeat proteins principally expressed in the brain. The Journal of biological chemistry 275: 19970-19977.

Chambliss, K.L., I.S. Yuhanna, C. Mineo, P. Liu, Z. German, T.S. Sherman, M.E. Mendelsohn, R.G. Anderson \& P.W. Shaul, (2000) Estrogen receptor alpha and endothelial nitric oxide synthase are organized into a functional signaling module in caveolae. Circulation research 87: E44-52.

Chen, H.W., M.J. Marinissen, S.W. Oh, X. Chen, M. Melnick, N. Perrimon, J.S. Gutkind \& S.X. Hou, (2002) CKA, a novel multidomain protein, regulates the JUN N-terminal kinase signal transduction pathway in Drosophila. Molecular and cellular biology 22: 1792-1803.

Chen, X. \& H. Ding, (2011) Increased expression of the tail-anchored membrane protein SLMAP in adipose tissue from type 2 Tally Ho diabetic mice. Experimental diabetes research 2011: 421982.

Chen, Y.K., C.Y. Chen, H.T. Hu \& Y.P. Hsueh, (2012) CTTNBP2, but not CTTNBP2NL, regulates dendritic spinogenesis and synaptic distribution of the striatin-PP2A complex. Molecular biology of the cell 23: 4383-4392.

Chen, Y.K. \& Y.P. Hsueh, (2012) Cortactin-binding protein 2 modulates the mobility of cortactin and regulates dendritic spine formation and maintenance. The Journal of neuroscience : the official journal of the Society for Neuroscience 32: 1043-1055.

Cheung, J., E. Petek, K. Nakabayashi, L.C. Tsui, J.B. Vincent \& S.W. Scherer, (2001) Identification of the human cortactin-binding protein-2 gene from the autism candidate region at $7 q 31$. Genomics 78: 7-11.

Chin, D. \& A. Means, (2000) Calmodulin: a prototypical calcium sensor. Trends in cell biology 10: 322328.

Cho, U.S. \& W. Xu, (2007) Crystal structure of a protein phosphatase 2A heterotrimeric holoenzyme. Nature 445: 53-57.

Choudhary, C., C. Kumar, F. Gnad, M.L. Nielsen, M. Rehman, T.C. Walther, J.V. Olsen \& M. Mann, (2009) Lysine acetylation targets protein complexes and co-regulates major cellular functions. Science 325: 834-840.

Chow, A., Y. Hao \& X. Yang, (2010) Molecular characterization of human homologs of yeast MOB1. International journal of cancer. Journal international du cancer 126: 2079-2089.

Clatterbuck, R.E., C.G. Eberhart, B.J. Crain \& D. Rigamonti, (2001) Ultrastructural and immunocytochemical evidence that an incompetent blood-brain barrier is related to the pathophysiology of cavernous malformations. Journal of neurology, neurosurgery, and psychiatry 71: 188-192.

Colot, H.V., G. Park, G.E. Turner, C. Ringelberg, C.M. Crew, L. Litvinkova, R.L. Weiss, K.A. Borkovich \& J.C. Dunlap, (2006) A high-throughput gene knockout procedure for Neurospora reveals functions for multiple transcription factors. Proc. Natl. Acad. Sci. U S A 103: 10352-10357.

Cosen-Binker, L.I. \& A. Kapus, (2006) Cortactin: the gray eminence of the cytoskeleton. Physiology 21: 352-361.

Couet, J., S. Li, T. Okamoto, T. Ikezu \& M.P. Lisanti, (1997) Identification of peptide and protein ligands for the caveolin-scaffolding domain. Implications for the interaction of caveolin with caveolaeassociated proteins. The Journal of biological chemistry 272: 6525-6533.

Dan, I., S.E. Ong, N.M. Watanabe, B. Blagoev, M.M. Nielsen, E. Kajikawa, T.Z. Kristiansen, M. Mann \& A. Pandey, (2002) Cloning of MASK, a novel member of the mammalian germinal center kinase III subfamily, with apoptosis-inducing properties. The Journal of biological chemistry 277: 59295939.

Dan, I., N.M. Watanabe, T. Kobayashi, K. Yamashita-Suzuki, Y. Fukagaya, E. Kajikawa, W.K. Kimura, T.M. Nakashima, K. Matsumoto, J. Ninomiya-Tsuji \& A. Kusumi, (2000) Molecular cloning of MINK, a 
novel member of mammalian GCK family kinases, which is up-regulated during postnatal mouse cerebral development. FEBS letters 469: 19-23.

Dan, I., N.M. Watanabe \& A. Kusumi, (2001) The Ste20 group kinases as regulators of MAP kinase cascades. Trends in cell biology 11: 220-230.

De Groot, P.W., K.J. Hellingwerf \& F.M. Klis, (2003) Genome-wide identification of fungal GPI proteins. Yeast 20: 781-796.

De Sampaio, G., J.P. Bourdineaud \& G.J. Lauquin, (1999) A constitutive role for GPI-anchors in Saccharomyces cerevisiae: cell wall targeting. Molecular microbiology 34: 247-256.

Delaval, B. \& S.J. Doxsey, (2010) Pericentrin in cellular function and disease. The Journal of cell biology 188: $181-190$.

Delgado-Alvarez, D.L., S. Bartnicki-Garcia, S. Seiler \& R.R. Mourino-Perez, (2014) Septum development in Neurospora crassa: the septal actomyosin tangle. PloS one 9: e96744.

Delpire, E., (2009) The mammalian family of sterile 20p-like protein kinases. Pflugers Arch - Eur J Physiol 458: 953-967.

Delpire, E., (2009) The mammalian family of sterile 20p-like protein kinases. Pflugers Archiv : European journal of physiology 458: 953-967.

Dettmann, A., Y. Heilig, S. Ludwig, K. Schmitt, J. Illgen, A. Fleissner, O. Valerius \& S. Seiler, (2013) HAM-2 and HAM-3 are central for the assembly of the Neurospora STRIPAK complex at the nuclear envelope and regulate nuclear accumulation of the MAP kinase MAK-1 in a MAK-2-dependent manner. Molecular microbiology 90: 796-812.

Dettmann, A., Y. Heilig, O. Valerius, S. Ludwig \& S. Seiler, (2014) Fungal Communication Requires the MAK-2 Pathway Elements STE-20 and RAS-2, the NRC-1 Adapter STE-50 and the MAP Kinase Scaffold HAM-5. PLoS genetics 10: e1004762.

Dettmann, A., J. Illgen, S. Marz, T. Schurg, A. Fleissner \& S. Seiler, (2012) The NDR kinase scaffold HYM1/MO25 is essential for MAK2 map kinase signaling in Neurospora crassa. PLoS genetics 8: e1002950.

Dingledine, R., K. Borges, D. Bowie \& S.F. Traynelis, (1999) The glutamate receptor ion channels. Pharmacological reviews 51: 7-61.

Dinur-Mills, M., M. Tal \& O. Pines, (2008) Dual Targeted Mitochondrial Proteins Are Characterized by Lower MTS Parameters and Total Net Charge. PloS one 3: e2161.

Dirschnabel, D.E., M. Nowrousian, N. Cano-Domínguez, J. Aguirre, I. Teichert \& U. Kück, (2014) New insights into the roles of NADPH oxidases in sexual development and ascospore germination in Sordaria macrospora. Genetics 196: 729-744.

Djordjevic, J.T., M. Del Poeta, T.C. Sorrell, K.M. Turner \& L.C. Wright, (2005) Secretion of cryptococcal phospholipase B1 (PLB1) is regulated by a glycosylphosphatidylinositol (GPI) anchor. The Biochemical journal 389: 803-812.

Dvash, E., G. Kra-Oz, C. Ziv, S. Carmeli \& O. Yarden, (2010) The NDR kinase DBF-2 is involved in regulation of mitosis, conidial development, and glycogen metabolism in Neurospora crassa. Eukaryotic cell 9: 502-513.

Eisenhaber, B., P. Bork \& F. Eisenhaber, (1998) Sequence properties of GPI-anchored proteins near the omega-site: constraints for the polypeptide binding site of the putative transamidase. Protein Engineering 11: 1155-1161.

Eisenhaber, B., P. Bork \& F. Eisenhaber, (1999) Prediction of potential GPI-modification sites in proprotein sequences. Journal of molecular biology 292: 741-758.

Eisenhaber, B., P. Bork \& F. Eisenhaber, (2001) Post-translational GPI lipid anchor modification of proteins in kingdoms of life: analysis of protein sequence data from complete genomes. Protein engineering 14: 17-25. 
Eisenhaber, B., P. Bork, Y. Yuan, G. Loffler \& F. Eisenhaber, (2000) Automated annotation of GPI-anchor sites: case study C. elegans. Trends in biochemical sciences 25: 340-341.

Elleuche, S. \& S. Pöggeler, (2009) Beta-carbonic anhydrases play a role in fruiting-body development and ascospore germination in the filamentous fungus Sordaria macrospora. PloS one 4: e5177.

Emanuelsson, O., S. Brunak, G. von Heijne \& H. Nielsen, (2007) Locating proteins in the cell using TargetP, SignalP and related tools. Nature protocols 2: 953-971.

Engh, I., M. Nowrousian \& U. Kück, (2010) Sordaria macrospora, a model organism to study fungal cellular development. European journal of cell biology 89: 864-872.

Engh, I., C. Würtz, K. Witzel-Schlömp, H.Y. Zhang, B. Hoff, M. Nowrousian, H. Rottensteiner \& U. Kück, (2007) The WW domain protein PRO40 is required for fungal fertility and associates with Woronin bodies. Eukaryot. Cell 6: 831-843.

Esser, K., (1992) Cryptogams-Cyanaobacteria, Fungi, Algae and Lichens. Cambridge University Press, London.

Ewing, R.M., P. Chu, F. Elisma, H. Li, P. Taylor, S. Climie, L. McBroom-Cerajewski, M.D. Robinson, L. O'Connor, M. Li, R. Taylor, M. Dharsee, Y. Ho, A. Heilbut, L. Moore, S. Zhang, O. Ornatsky, Y.V. Bukhman, M. Ethier, Y. Sheng, J. Vasilescu, M. Abu-Farha, J.P. Lambert, H.S. Duewel, Stewart, II, B. Kuehl, K. Hogue, K. Colwill, K. Gladwish, B. Muskat, R. Kinach, S.L. Adams, M.F. Moran, G.B. Morin, T. Topaloglou \& D. Figeys, (2007) Large-scale mapping of human protein-protein interactions by mass spectrometry. Molecular systems biology 3: 89.

Fazioli, F., L. Minichiello, B. Matoskova, W.T. Wong \& P.P. Di Fiore, (1993) Eps15, a novel tyrosine kinase substrate, exhibits transforming activity. Molecular and cellular biology 13: 5814-5828.

Felsenstein, J., (2013) PHYLIP (Phylogeny Inference Package) version 3.695.

Fidalgo, M., A. Guerrero, M. Fraile, C. Iglesias, C.M. Pombo \& J. Zalvide, (2012) Adaptor protein cerebral cavernous malformation 3 (CCM3) mediates phosphorylation of the cytoskeletal proteins Ezrin/Radixin/Moesin by mammalian Ste20-4 to protect cells from oxidative stress. The Journal of biological chemistry 287: 11556-11565.

Fleißner, A., A.C. Leeder, M.G. Roca, N.D. Read \& N.L. Glass, (2009) Oscillatory recruitment of signaling proteins to cell tips promotes coordinated behavior during cell fusion. Proceedings of the National Academy of Sciences of the United States of America 106: 19387-19392.

Fleißner, A., A. Simonin \& N.L. Glass, (2008) Cell Fusion in the Filamentous Fungus, Neurospora crassa. In: Cell Fusion. E. Chen (ed). Humana Press, pp. 21-38.

Frieman, M.B. \& B.P. Cormack, (2003) The omega-site sequence of glycosylphosphatidylinositolanchored proteins in Saccharomyces cerevisiae can determine distribution between the membrane and the cell wall. Molecular microbiology 50: 883-896.

Frieman, M.B. \& B.P. Cormack, (2004) Multiple sequence signals determine the distribution of glycosylphosphatidylinositol proteins between the plasma membrane and cell wall in Saccharomyces cerevisiae. Microbiology 150: 3105-3114.

Frost, A., M.G. Elgort, O. Brandman, C. Ives, S.R. Collins, L. Miller-Vedam, J. Weibezahn, M.Y. Hein, I. Poser, M. Mann, A.A. Hyman \& J.S. Weissman, (2012) Functional repurposing revealed by comparing S. pombe and S. cerevisiae genetic interactions. Cell 149: 1339-1352.

Fu, C., P. Iyer, A. Herkal, J. Abdullah, A. Stout \& S.J. Free, (2011) Identification and characterization of genes required for cell-to-cell fusion in Neurospora crassa. Eukaryotic cell 10: 1100-1109.

Fujita, M. \& T. Kinoshita, (2012) GPI-anchor remodeling: potential functions of GPI-anchors in intracellular trafficking and membrane dynamics. Biochimica et Biophysica Acta (BBA) Molecular and Cell Biology of Lipids 1821: 1050-1058.

Gaillard, S., Y. Bailly, M. Benoist, T. Rakitina, J.P. Kessler, L. Fronzaroli-Molinieres, B. Dargent \& F. Castets, (2006) Targeting of proteins of the striatin family to dendritic spines: role of the coiledcoil domain. Traffic 7: 74-84. 
Gaillard, S., M. Bartoli, F. Castets \& A. Monneron, (2001) Striatin, a calmodulin-dependent scaffolding protein, directly binds caveolin-1. FEBS letters 508: 49-52.

Galian, C., P. Bjorkholm, N. Bulleid \& G. von Heijne, (2012) Efficient glycosylphosphatidylinositol (GPI) modification of membrane proteins requires a C-terminal anchoring signal of marginal hydrophobicity. The Journal of biological chemistry 287: 16399-16409.

Gilbert, N.M., L.G. Baker, C.A. Specht \& J.K. Lodge, (2012) A glycosylphosphatidylinositol anchor is required for membrane localization but dispensable for cell wall association of chitin deacetylase 2 in Cryptococcus neoformans. mBio 3.

Gordon, J., J. Hwang, K. Carrier, C. Jones, Q. Kern, C. Moreno, R. Karas \& D. Pallas, (2011) Protein phosphatase 2a (PP2A) binds within the oligomerization domain of striatin and regulates the phosphorylation and activation of the mammalian Ste20-Like kinase Mst3. BMC biochemistry 12: 54.

Goudreault, M., L.M. D'Ambrosio, M.J. Kean, M.J. Mullin, B.G. Larsen, A. Sanchez, S. Chaudhry, G.I. Chen, F. Sicheri, A.I. Nesvizhskii, R. Aebersold, B. Raught \& A.C. Gingras, (2009) A PP2A phosphatase high density interaction network identifies a novel striatin-interacting phosphatase and kinase complex linked to the cerebral cavernous malformation 3 (CCM3) protein. Molecular \& cellular proteomics : MCP 8: 157-171.

Greenman, C., P. Stephens, R. Smith, G.L. Dalgliesh, C. Hunter, G. Bignell, H. Davies, J. Teague, A. Butler, C. Stevens, S. Edkins, S. O'Meara, I. Vastrik, E.E. Schmidt, T. Avis, S. Barthorpe, G. Bhamra, G. Buck, B. Choudhury, J. Clements, J. Cole, E. Dicks, S. Forbes, K. Gray, K. Halliday, R. Harrison, K. Hills, J. Hinton, A. Jenkinson, D. Jones, A. Menzies, T. Mironenko, J. Perry, K. Raine, D. Richardson, R. Shepherd, A. Small, C. Tofts, J. Varian, T. Webb, S. West, S. Widaa, A. Yates, D.P. Cahill, D.N. Louis, P. Goldstraw, A.G. Nicholson, F. Brasseur, L. Looijenga, B.L. Weber, Y.E. Chiew, A. DeFazio, M.F. Greaves, A.R. Green, P. Campbell, E. Birney, D.F. Easton, G. Chenevix-Trench, M.H. Tan, S.K. Khoo, B.T. Teh, S.T. Yuen, S.Y. Leung, R. Wooster, P.A. Futreal \& M.R. Stratton, (2007) Patterns of somatic mutation in human cancer genomes. Nature 446: 153-158.

Guclu, B., A.K. Ozturk, K.L. Pricola, K. Bilguvar, D. Shin, B.J. O'Roak \& M. Gunel, (2005) Mutations in apoptosis-related gene, PDCD10, cause cerebral cavernous malformation 3. Neurosurgery 57: 1008-1013.

Guo, Z., (2013) Synthetic Studies of Glycosylphosphatidylinositol (GPI) Anchors and GPI-Anchored Peptides, Glycopeptides, and Proteins. Current organic synthesis 10: 366-383.

Guzzo, R.M., M. Salih, E.D. Moore \& B.S. Tuana, (2005) Molecular properties of cardiac tail-anchored membrane protein SLMAP are consistent with structural role in arrangement of excitationcontraction coupling apparatus. American journal of physiology. Heart and circulatory physiology 288: H1810-1819.

Guzzo, R.M., S. Sevinc, M. Salih \& B.S. Tuana, (2004) A novel isoform of sarcolemmal membraneassociated protein (SLMAP) is a component of the microtubule organizing centre. Journal of cell science 117: 2271-2281.

Guzzo, R.M., J. Wigle, M. Salih, E.D. Moore \& B.S. Tuana, (2004) Regulated expression and temporal induction of the tail-anchored sarcolemmal-membrane-associated protein is critical for myoblast fusion. The Biochemical journal 381: 599-608.

Hamada, K., H. Terashima, M. Arisawa, N. Yabuki \& K. Kitada, (1999) Amino acid residues in the omegaminus region participate in cellular localization of yeast glycosylphosphatidylinositol-attached proteins. Journal of bacteriology 181: 3886-3889.

Hanks, S.K. \& T. Hunter, (1995) Protein kinases 6. The eukaryotic protein kinase superfamily: kinase (catalytic) domain structure and classification. FASEB J 9: 576-596. 
Hao, Q., M. Feng, Z. Shi, C. Li, M. Chen, W. Wang, M. Zhang, S. Jiao \& Z. Zhou, (2014) Structural insights into regulatory mechanisms of MO25-mediated kinase activation. Journal of structural biology 186: 224-233.

He, Y., H. Zhang, L. Yu, M. Gunel, T.J. Boggon, H. Chen \& W. Min, (2010) Stabilization of VEGFR2 signaling by cerebral cavernous malformation 3 is critical for vascular development. Science signaling $\mathbf{3}$ : ra26.

Heilig, Y., A. Dettmann, R.R. Mourino-Perez, K. Schmitt, O. Valerius \& S. Seiler, (2014) Proper actin ring formation and septum constriction requires coordinated regulation of SIN and MOR pathways through the germinal centre kinase MST-1. PLoS genetics 10: e1004306.

Heilig, Y., K. Schmitt \& S. Seiler, (2013) Phospho-regulation of the Neurospora crassa septation initiation network. PloS one 8: e79464.

Hein, L., M.E. Stevens, G.S. Barsh, R.E. Pratt, B.K. Kobilka \& V.J. Dzau, (1997) Overexpression of angiotensin AT1 receptor transgene in the mouse myocardium produces a lethal phenotype associated with myocyte hyperplasia and heart block. Proceedings of the National Academy of Sciences of the United States of America 94: 6391-6396.

Hergovich, A., S.J. Bichsel \& B.A. Hemmings, (2005) Human NDR kinases are rapidly activated by MOB proteins through recruitment to the plasma membrane and phosphorylation. Molecular and cellular biology 25: 8259-8272.

Hergovich, A. \& B.A. Hemmings, (2012) Hippo signalling in the G2/M cell cycle phase: lessons learned from the yeast MEN and SIN pathways. Seminars in cell \& developmental biology 23: 794-802.

Herzog, F., A. Kahraman, D. Boehringer, R. Mak, A. Bracher, T. Walzthoeni, A. Leitner, M. Beck, F.U. Hartl, N. Ban, L. Malmstrom \& R. Aebersold, (2012) Structural probing of a protein phosphatase 2A network by chemical cross-linking and mass spectrometry. Science 337: 1348-1352.

Hoffman, C.S. \& F. Winston, (1987) A ten-minute DNA preparation from yeast efficiently releases autonomous plasmids for transformation of Escherichia coli. Gene 57: 267-272.

Hofmann, K. \& P. Bucher, (1995) The FHA domain: a putative nuclear signalling domain found in protein kinases and transcription factors. Trends in biochemical sciences 20: 347-349.

Holmes, K., O.L. Roberts, A.M. Thomas \& M.J. Cross, (2007) Vascular endothelial growth factor receptor2: structure, function, intracellular signalling and therapeutic inhibition. Cellular signalling 19: 2003-2012.

Hu, Y., C. Leo, S. Yu, B.C. Huang, H. Wang, M. Shen, Y. Luo, S. Daniel-Issakani, D.G. Payan \& X. Xu, (2004) Identification and functional characterization of a novel human misshapen/Nck interacting kinase-related kinase, hMINK beta. The Journal of biological chemistry 279: 54387-54397.

Huang, C.Y., Y.M. Wu, C.Y. Hsu, W.S. Lee, M.D. Lai, T.J. Lu, C.L. Huang, T.H. Leu, H.M. Shih, H.I. Fang, D.R. Robinson, H.J. Kung \& C.J. Yuan, (2002) Caspase activation of mammalian sterile 20-like kinase 3 (Mst3). Nuclear translocation and induction of apoptosis. The Journal of biological chemistry 277: 34367-34374.

Huang, J., T. Liu, L.G. Xu, D. Chen, Z. Zhai \& H.B. Shu, (2005) SIKE is an IKK epsilon/TBK1-associated suppressor of TLR3- and virus-triggered IRF-3 activation pathways. The EMBO journal 24: 40184028.

Hwang, J. \& D.C. Pallas, (2014) STRIPAK complexes: structure, biological function, and involvement in human diseases. The international journal of biochemistry \& cell biology 47: 118-148.

Hyodo, T., S. Ito, H. Hasegawa, E. Asano, M. Maeda, T. Urano, M. Takahashi, M. Hamaguchi \& T. Senga, (2012) Misshapen-like kinase 1 (MINK1) is a novel component of striatin-interacting phosphatase and kinase (STRIPAK) and is required for the completion of cytokinesis. The Journal of biological chemistry 287: 25019-25029.

llangumaran, S., H.T. He \& D.C. Hoessli, (2000) Microdomains in lymphocyte signalling: beyond GPIanchored proteins. Immunology today 21: 2-7. 
Ip, Y.T. \& R.J. Davis, (1998) Signal transduction by the c-Jun N-terminal kinase (JNK)--from inflammation to development. Current opinion in cell biology 10: 205-219.

Jacobsen, S., M. Wittig \& S. Pöggeler, (2002) Interaction between mating-type proteins from the homothallic fungus Sordaria macrospora. Current genetics 41: 150-158.

Jones, D.R. \& I. Varela-Nieto, (1998) The role of glycosyl-phosphatidylinositol in signal transduction. The international journal of biochemistry \& cell biology 30: 313-326.

Kanai, M., K. Kume, K. Miyahara, K. Sakai, K. Nakamura, K. Leonhard, D.J. Wiley, F. Verde, T. Toda \& D. Hirata, (2005) Fission yeast MO25 protein is localized at SPB and septum and is essential for cell morphogenesis. The EMBO journal 24: 3012-3025.

Kaneko, S., X. Chen, P. Lu, X. Yao, T.G. Wright, M. Rajurkar, K. Kariya, J. Mao, Y.T. Ip \& L. Xu, (2011) Smad inhibition by the Ste20 kinase Misshapen. Proceedings of the National Academy of Sciences of the United States of America 108: 11127-11132.

Kawabata, T. \& H. Inoue, (2007) Detection of physical interactions by immunoprecipitation of FLAG- and HA-tagged proteins expressed at the his-3 locus in Neurospora crassa. Fungal Genetics Newsletter.

Kean, M.J., D.F. Ceccarelli, M. Goudreault, M. Sanches, S. Tate, B. Larsen, L.C. Gibson, W.B. Derry, I.C. Scott, L. Pelletier, G.S. Baillie, F. Sicheri \& A.C. Gingras, (2011) Structure-function analysis of core STRIPAK Proteins: a signaling complex implicated in Golgi polarization. The Journal of biological chemistry 286: 25065-25075.

Kinoshita, T., (2014) Enzymatic mechanism of GPI-anchor attachment clarified. Cell Cycle 13: 1838-1839.

Klix, V., M. Nowrousian, C. Ringelberg, J.J. Loros, J.C. Dunlap \& S. Pöggeler, (2010) Functional characterization of MAT1-1-specific mating-type genes in the homothallic ascomycete Sordaria macrospora provides new insights into essential and nonessential sexual regulators. Eukaryotic cell 9: 894-905.

Koch, M.R. \& L. Pillus, (2009) The glucanosyltransferase Gas1 functions in transcriptional silencing. Proceedings of the National Academy of Sciences 106: 11224-11229.

Kosugi, S., M. Hasebe, M. Tomita \& H. Yanagawa, (2009) Systematic identification of cell cycledependent yeast nucleocytoplasmic shuttling proteins by prediction of composite motifs. Proceedings of the National Academy of Sciences 106: 10171-10176.

Krapp, A. \& V. Simanis, (2008) An overview of the fission yeast septation initiation network (SIN). Biochemical Society transactions 36: 411-415.

Krishnan, K.S., R. Rikhy, S. Rao, M. Shivalkar, M. Mosko, R. Narayanan, P. Etter, P.S. Estes \& M. Ramaswami, (2001) Nucleoside diphosphate kinase, a source of GTP, is required for dynamindependent synaptic vesicle recycling. Neuron 30: 197-210.

Kück, U., S. Pöggeler, M. Nowrousian, N. Nolting \& I. Engh, (2009) Sordaria macrospora, a model system for fungal development., p. 17-39. Springer Verlag, Heidelberg, New York.

Kulkarni, R.D., H.S. Kelkar \& R.A. Dean, (2003) An eight-cysteine-containing CFEM domain unique to a group of fungal membrane proteins. Trends in biochemical sciences 28: 118-121.

la Cour, T., L. Kiemer, A. Mølgaard, R. Gupta, K. Skriver \& S. Brunak, (2004) Analysis and prediction of leucine-rich nuclear export signals. Protein Eng. Des. Sel. 17: 527-536.

Laemmli, U.K., (1970) Cleavage of structural proteins during the assembly of the head of bacteriophage T4. Nature 227: 680-685.

Lalli, E. \& P. Sassone-Corsi, (1994) Signal transduction and gene regulation: the nuclear response to cAMP. The Journal of biological chemistry 269: 17359-17362.

Lankes, W., A. Griesmacher, J. Grunwald, R. Schwartz-Albiez \& R. Keller, (1988) A heparin-binding protein involved in inhibition of smooth-muscle cell proliferation. The Biochemical journal 251: 831-842. 
Larkin, M.A., G. Blackshields, N.P. Brown, R. Chenna, P.A. McGettigan, H. McWilliam, F. Valentin, I.M. Wallace, A. Wilm, R. Lopez, J.D. Thompson, T.J. Gibson \& D.G. Higgins, (2007) Clustal W and Clustal X version 2.0. Bioinformatics 23: 2947-2948.

Lecellier, G. \& P. Silar, (1994) Rapid methods for nucleic acids extraction from Petri dish-grown mycelia. Current genetics 25: 122-123.

Lechward, K., O.S. Awotunde, W. Swiatek \& G. Muszynska, (2001) Protein phosphatase 2A: variety of forms and diversity of functions. Acta biochimica Polonica 48: 921-933.

Lee, W.S., C.Y. Hsu, P.L. Wang, C.Y. Huang, C.H. Chang \& C.J. Yuan, (2004) Identification and characterization of the nuclear import and export signals of the mammalian Ste20-like protein kinase 3. FEBS Lett. 572: 41-45.

Li, D. \& R. Roberts, (2001) WD-repeat proteins: structure characteristics, biological function, and their involvement in human diseases. Cellular and molecular life sciences : CMLS 58: 2085-2097.

Li, E. \& K. Hristova, (2006) Role of receptor tyrosine kinase transmembrane domains in cell signaling and human pathologies. Biochemistry 45: 6241-6251.

Li, S., J. Couet \& M.P. Lisanti, (1996) Src tyrosine kinases, Galpha subunits, and H-Ras share a common membrane-anchored scaffolding protein, caveolin. Caveolin binding negatively regulates the auto-activation of Src tyrosine kinases. The Journal of biological chemistry 271: 29182-29190.

Li, X., R. Zhang, H. Zhang, Y. He, W. Ji, W. Min \& T.J. Boggon, (2010) Crystal structure of CCM3, a cerebral cavernous malformation protein critical for vascular integrity. The Journal of biological chemistry 285: 24099-24107.

Lieber, T., S. Kidd, E. Alcamo, V. Corbin \& M.W. Young, (1993) Antineurogenic phenotypes induced by truncated Notch proteins indicate a role in signal transduction and may point to a novel function for Notch in nuclei. Genes \& development 7: 1949-1965.

Lin, A., A. Hokugo, J. Choi \& I. Nishimura, (2010) Small cytoskeleton-associated molecule, fibroblast growth factor receptor 1 oncogene partner 2/wound inducible transcript-3.0 (FGFR1OP2/wit3.0), facilitates fibroblast-driven wound closure. The American journal of pathology 176: 108-121.

Lin, J.L., H.C. Chen, H.I. Fang, D. Robinson, H.J. Kung \& H.M. Shih, (2001) MST4, a new Ste20-related kinase that mediates cell growth and transformation via modulating ERK pathway. Oncogene 20: 6559-6569.

Linding, R., R.B. Russell, V. Neduva \& T.J. Gibson, (2003) GlobPlot: Exploring protein sequences for globularity and disorder. Nucleic acids research 31: 3701-3708.

Lisa-Santamaria, P., A. Jimenez \& J.L. Revuelta, (2012) The protein factor-arrest 11 (Far11) is essential for the toxicity of human caspase-10 in yeast and participates in the regulation of autophagy and the DNA damage signaling. The Journal of biological chemistry 287: 29636-29647.

Liu, J., Q. Zheng, Y. Deng, C.S. Cheng, N.R. Kallenbach \& M. Lu, (2006) A seven-helix coiled coil. Proceedings of the National Academy of Sciences of the United States of America 103: 1545715462.

Lorber, B., M.L. Howe, L.I. Benowitz \& N. Irwin, (2009) Mst3b, an Ste20-like kinase, regulates axon regeneration in mature CNS and PNS pathways. Nature neuroscience 12: 1407-1414.

Lord, K.M. \& N.D. Read, (2011) Perithecium morphogenesis in Sordaria macrospora. Fungal Genetics and Biology 48: 388-399.

Low, M.G., (1989) Glycosyl-phosphatidylinositol: a versatile anchor for cell surface proteins. FASEB J 3: 1600-1608.

Lu, Q., D.C. Pallas, H.K. Surks, W.E. Baur, M.E. Mendelsohn \& R.H. Karas, (2004) Striatin assembles a membrane signaling complex necessary for rapid, nongenomic activation of endothelial NO synthase by estrogen receptor alpha. Proceedings of the National Academy of Sciences of the United States of America 101: 17126-17131. 
Lu, T.J., W.Y. Lai, C.Y. Huang, W.J. Hsieh, J.S. Yu, Y.J. Hsieh, W.T. Chang, T.H. Leu, W.C. Chang, W.J. Chuang, M.J. Tang, T.Y. Chen, T.L. Lu \& M.D. Lai, (2006) Inhibition of cell migration by autophosphorylated mammalian sterile 20-like kinase 3 (MST3) involves paxillin and proteintyrosine phosphatase-PEST. The Journal of biological chemistry 281: 38405-38417.

Luca, F.C., M. Mody, C. Kurischko, D.M. Roof, T.H. Giddings \& M. Winey, (2001) Saccharomyces cerevisiae Mob1p is required for cytokinesis and mitotic exit. Molecular and cellular biology 21: 6972-6983.

Ma, H.L., Y.L. Peng, L. Gong, W.B. Liu, S. Sun, J. Liu, C.B. Zheng, H. Fu, D. Yuan, J. Zhao, P.C. Chen, S.S. Xie, X.M. Zeng, Y.M. Xiao, Y. Liu \& D.W. Li, (2009) The goldfish SG2NA gene encodes two alpha-type regulatory subunits for PP2A and displays distinct developmental expression pattern. Gene regulation and systems biology 3: 115-129.

Ma, X., H. Zhao, J. Shan, F. Long, Y. Chen, Y. Zhang, X. Han \& D. Ma, (2007) PDCD10 interacts with Ste20related kinase MST4 to promote cell growth and transformation via modulation of the ERK pathway. Molecular biology of the cell 18: 1965-1978.

Maddi, A., A. Dettman, C. Fu, S. Seiler \& S.J. Free, (2012) WSC-1 and HAM-7 Are MAK-1 MAP Kinase Pathway Sensors Required for Cell Wall Integrity and Hyphal Fusion in Neurospora crassa. PloS one 7: e42374.

Maerz, S., A. Dettmann, C. Ziv, Y. Liu, O. Valerius, O. Yarden \& S. Seiler, (2009) Two NDR kinase-MOB complexes function as distinct modules during septum formation and tip extension in Neurospora crassa. Molecular microbiology 74: 707-723.

Mahlert, M., L. Leveleki, A. Hlubek, B. Sandrock \& M. Bolker, (2006) Rac1 and Cdc42 regulate hyphal growth and cytokinesis in the dimorphic fungus Ustilago maydis. Molecular microbiology 59: 567-578.

Maller, J.L., (2003) Signal transduction. Fishing at the cell surface. Science 300: 594-595.

Marchler-Bauer, A., S. Lu, J.B. Anderson, F. Chitsaz, M.K. Derbyshire, C. DeWeese-Scott, J.H. Fong, L.Y. Geer, R.C. Geer, N.R. Gonzales, M. Gwadz, D.I. Hurwitz, J.D. Jackson, Z. Ke, C.J. Lanczycki, F. Lu, G.H. Marchler, M. Mullokandov, M.V. Omelchenko, C.L. Robertson, J.S. Song, N. Thanki, R.A. Yamashita, D. Zhang, N. Zhang, C. Zheng \& S.H. Bryant, (2011) CDD: a Conserved Domain Database for the functional annotation of proteins. Nucleic acids research 39: D225-D229.

Martindale, J.L. \& N.J. Holbrook, (2002) Cellular response to oxidative stress: signaling for suicide and survival. Journal of cellular physiology 192: 1-15.

Masloff, S., S. Pöggeler \& U. Kück, (1999) The pro1(+) gene from Sordaria macrospora encodes a C6 zinc finger transcription factor required for fruiting body development. Genetics 152: 191-199.

Mason, J.M. \& K.M. Arndt, (2004) Coiled coil domains: stability, specificity, and biological implications. Chembiochem : a European journal of chemical biology 5: 170-176.

Matsuki, T., J. Chen \& B.W. Howell, (2013) Acute inactivation of the serine-threonine kinase Stk25 disrupts neuronal migration. Neural development 8: 21.

Mayor, S. \& H. Riezman, (2004) Sorting GPI-anchored proteins. Nature Reviews Molecular Cell Biology: 110-120.

Mendoza, M., S. Redemann \& D. Brunner, (2005) The fission yeast M025 protein functions in polar growth and cell separation. European journal of cell biology 84: 915-926.

Moqrich, A., M.G. Mattei, M. Bartoli, T. Rakitina, G. Baillat, A. Monneron \& F. Castets, (1998) Cloning of human striatin cDNA (STRN), gene mapping to 2p22-p21, and preferential expression in brain. Genomics 51: 136-139.

Moreno, C.S., W.S. Lane \& D.C. Pallas, (2001) A mammalian homolog of yeast MOB1 is both a member and a putative substrate of striatin family-protein phosphatase $2 \mathrm{~A}$ complexes. The Journal of biological chemistry 276: 24253-24260. 
Moreno, C.S., S. Park, K. Nelson, D. Ashby, F. Hubalek, W.S. Lane \& D.C. Pallas, (2000) WD40 repeat proteins striatin and $S / G(2)$ nuclear autoantigen are members of a novel family of calmodulinbinding proteins that associate with protein phosphatase $2 \mathrm{~A}$. The Journal of biological chemistry 275: 5257-5263.

Morrell, J.L., G.C. Tomlin, S. Rajagopalan, S. Venkatram, A.S. Feoktistova, J.J. Tasto, S. Mehta, J.L. Jennings, A. Link, M.K. Balasubramanian \& K.L. Gould, (2004) Sid4p-Cdc11p assembles the septation initiation network and its regulators at the S. pombe SPB. Cur. Biol. 14: 579-584.

Mosavi, L.K., T.J. Cammett, D.C. Desrosiers \& Z.Y. Peng, (2004) The ankyrin repeat as molecular architecture for protein recognition. Protein science : a publication of the Protein Society 13: $1435-1448$.

Muro, Y., E.K. Chan, G. Landberg \& E.M. Tan, (1995) A cell-cycle nuclear autoantigen containing WD-40 motifs expressed mainly in S and G2 phase cells. Biochemical and biophysical research communications 207: 1029-1037.

Neer, E.J., C.J. Schmidt, R. Nambudripad \& T.F. Smith, (1994) The ancient regulatory-protein family of WD-repeat proteins. Nature 371: 297-300.

Nicholas, K.B., H.B. Nicholas \& D.W. Deerfield, (1997) GeneDoc: Analysis and Visualization of Genetic Variation. EMBNEWnews 4.

Nogueira, E., M. Fidalgo, A. Molnar, J. Kyriakis, T. Force, J. Zalvide \& C.M. Pombo, (2008) SOK1 translocates from the Golgi to the nucleus upon chemical anoxia and induces apoptotic cell death. The Journal of biological chemistry 283: 16248-16258.

Nonaka, H., K. Takei, M. Umikawa, M. Oshiro, K. Kuninaka, M. Bayarjargal, T. Asato, Y. Yamashiro, Y. Uechi, S. Endo, T. Suzuki \& K. Kariya, (2008) MINK is a Rap2 effector for phosphorylation of the postsynaptic scaffold protein TANC1. Biochemical and biophysical research communications 377: 573-578.

Nordzieke, S., T. Zobel, B. Franzel, D.A. Wolters, U. Kück \& I. Teichert, (2014) A fungal SLMAP homolog plays a fundamental role in development and localizes to the nuclear envelope, ER, and mitochondria. Eukaryotic cell.

Nowrousian, M., (2010) Next-generation sequencing techniques for eukaryotic microorganisms: sequencing-based solutions to biological problems. Eukaryot. Cell 9: 1300-1310.

Nowrousian, M. \& P. Cebula, (2005) The gene for a lectin-like protein is transcriptionally activated during sexual development, but is not essential for fruiting-body formation in the filamentous fungus Sordaria macrospora. BMC microbiology 5: 64.

Nowrousian, M., S. Frank, S. Koers, P. Strauch, T. Weitner, C. Ringelberg, J.C. Dunlap, J.J. Loros \& U. Kück, (2007) The novel ER membrane protein PRO41 is essential for sexual development in the filamentous fungus Sordaria macrospora. Mol. Microbiol. 64: 923-937.

Nowrousian, M., S. Masloff, S. Pöggeler \& U. Kück, (1999) Cell differentiation during sexual development of the fungus Sordaria macrospora requires ATP citrate lyase activity. Molecular and cellular biology 19: 450-460.

Nowrousian, M., C. Ringelberg, J.C. Dunlap, J.J. Loros \& U. Kück, (2005) Cross-species microarray hybridization to identify developmentally regulated genes in the filamentous fungus Sordaria macrospora. Mol. Genet. Genomics 273: 137-149.

Nowrousian, M., J.E. Stajich, M. Chu, I. Engh, E. Espagne, K. Halliday, J. Kamerewerd, F. Kempken, B. Knab, H.C. Kuo, H.D. Osiewacz, S. Pöggeler, N.D. Read, S. Seiler, K.M. Smith, D. Zickler, U. Kück \& M. Freitag, (2010) De novo assembly of a $40 \mathrm{Mb}$ eukaryotic genome from short sequence reads: Sordaria macrospora, a model organism for fungal morphogenesis. PLoS genetics 6: e1000891.

Nowrousian, M., I. Teichert, S. Masloff \& U. Kuck, (2012) Whole-Genome Sequencing of Sordaria macrospora Mutants Identifies Developmental Genes. G3 (Bethesda) 2: 261-270. 
Nowrousian, M., C. Würtz, S. Pöggeler \& U. Kück, (2004) Comparative sequence analysis of Sordaria macrospora and Neurospora crassa as a means to improve genome annotation. Fungal Genet. Biol. 41: 285-292.

O'Lone, R., M.C. Frith, E.K. Karlsson \& U. Hansen, (2004) Genomic targets of nuclear estrogen receptors. Molecular endocrinology 18: 1859-1875.

Olsen, J.V., B. Blagoev, F. Gnad, B. Macek, C. Kumar, P. Mortensen \& M. Mann, (2006) Global, in vivo, and site-specific phosphorylation dynamics in signaling networks. Cell 127: 635-648.

Orlean, P. \& A.K. Menon, (2007) Thematic review series: lipid posttranslational modifications. GPIanchoring of protein in yeast and mammalian cells, or: how we learned to stop worrying and love glycophospholipids. Journal of lipid research 48: 993-1011.

Ota, T., Y. Suzuki, T. Nishikawa, T. Otsuki, T. Sugiyama, R. Irie, A. Wakamatsu, K. Hayashi, H. Sato, K. Nagai, K. Kimura, H. Makita, M. Sekine, M. Obayashi, T. Nishi, T. Shibahara, T. Tanaka, S. Ishii, J. Yamamoto, K. Saito, Y. Kawai, Y. Isono, Y. Nakamura, K. Nagahari, K. Murakami, T. Yasuda, T. Iwayanagi, M. Wagatsuma, A. Shiratori, H. Sudo, T. Hosoiri, Y. Kaku, H. Kodaira, H. Kondo, M. Sugawara, M. Takahashi, K. Kanda, T. Yokoi, T. Furuya, E. Kikkawa, Y. Omura, K. Abe, K. Kamihara, N. Katsuta, K. Sato, M. Tanikawa, M. Yamazaki, K. Ninomiya, T. Ishibashi, H. Yamashita, K. Murakawa, K. Fujimori, H. Tanai, M. Kimata, M. Watanabe, S. Hiraoka, Y. Chiba, S. Ishida, Y. Ono, S. Takiguchi, S. Watanabe, M. Yosida, T. Hotuta, J. Kusano, K. Kanehori, A. Takahashi-Fujii, H. Hara, T.O. Tanase, Y. Nomura, S. Togiya, F. Komai, R. Hara, K. Takeuchi, M. Arita, N. Imose, K. Musashino, H. Yuuki, A. Oshima, N. Sasaki, S. Aotsuka, Y. Yoshikawa, H. Matsunawa, T. Ichihara, N. Shiohata, S. Sano, S. Moriya, H. Momiyama, N. Satoh, S. Takami, Y. Terashima, O. Suzuki, S. Nakagawa, A. Senoh, H. Mizoguchi, Y. Goto, F. Shimizu, H. Wakebe, H. Hishigaki, T. Watanabe, A. Sugiyama, et al., (2004) Complete sequencing and characterization of 21,243 full-length human cDNAs. Nature genetics 36: 40-45.

Otten, P., G.P. Pizzolato, B. Rilliet \& J. Berney, (1989) [131 cases of cavernous angioma (cavernomas) of the CNS, discovered by retrospective analysis of 24,535 autopsies]. Neuro-Chirurgie 35: 82-83, 128-131.

Ouyang, H., X. Chen, Y. Lu, I.B. Wilson, G. Tang, A. Wang \& C. Jin, (2013) One single basic amino acid at the omega-1 or omega-2 site is a signal that retains glycosylphosphatidylinositol-anchored protein in the plasma membrane of Aspergillus fumigatus. Eukaryotic cell 12: 889-899.

Page, R.D.M., (1996) Tree View: An application to display phylogenetic trees on personal computers. Computer applications in the biosciences: CABIOS 12: 357-358.

Paladino, S., S. Lebreton, S. Tivodar, V. Campana, R. Tempre \& C. Zurzolo, (2008) Different GPIattachment signals affect the oligomerisation of GPI-anchored proteins and their apical sorting. Journal of cell science 121: 4001-4007.

Park, G., J.A. Servin, G.E. Turner, L. Altamirano, H.V. Colot, P. Collopy, L. Litvinkova, L. Li, C.A. Jones, F.G. Diala, J.C. Dunlap \& K.A. Borkovich, (2011) Global analysis of serine-threonine protein kinase genes in Neurospora crassa. Eukaryotic cell 10: 1553-1564.

Parton, R.G. \& K. Simons, (2007) The multiple faces of caveolae. Nature reviews. Molecular cell biology 8 : 185-194.

Paulick, M.G. \& C.R. Bertozzi, (2008) The glycosylphosphatidylinositol anchor: a complex membraneanchoring structure for proteins. Biochemistry 47: 6991-7000.

Pelham, H.R., (1990) The retention signal for soluble proteins of the endoplasmic reticulum. Trends in biochemical sciences 15: 483-486.

Petersen, T.N., S. Brunak, G. von Heijne \& H. Nielsen, (2011) SignalP 4.0: discriminating signal peptides from transmembrane regions. Nature methods 8: 785-786.

Pfeiffer, N.V., D. Dirndorfer, S. Lang, U.K. Resenberger, L.M. Restelli, C. Hemion, M. Miesbauer, S. Frank, A. Neutzner, R. Zimmermann, K.F. Winklhofer \& J. Tatzelt, (2013) Structural features within the 
nascent chain regulate alternative targeting of secretory proteins to mitochondria. The EMBO journal 32: 1036-1051.

Pichlmair, A., O. Schulz, C.P. Tan, T.I. Naslund, P. Liljestrom, F. Weber \& C. Reis e Sousa, (2006) RIG-Imediated antiviral responses to single-stranded RNA bearing 5'-phosphates. Science 314: 9971001.

Pierleoni, A., P. Martelli \& R. Casadio, (2008) PredGPI: a GPI-anchor predictor. BMC bioinformatics 9: 392.

Pittet, M. \& A. Conzelmann, (2007) Biosynthesis and function of GPI proteins in the yeast Saccharomyces cerevisiae. Biochimica et biophysica acta 1771: 405-420.

Pöggeler, S. \& U. Kück, (2004) A WD40 repeat protein regulates fungal cell differentiation and can be replaced functionally by the mammalian homologue striatin. Eukaryotic cell 3: 232-240.

Pöggeler, S. \& U. Kück, (2006) Highly efficient generation of signal transduction knockout mutants using a fungal strain deficient in the mammalian ku70 ortholog. Gene 378: 1-10.

Pöggeler, S., S. Masloff, B. Hoff, S. Mayrhofer \& U. Kück, (2003) Versatile EGFP reporter plasmids for cellular localization of recombinant gene products in filamentous fungi. Current genetics 43: 5461.

Pöggeler, S., M. Nowrousian, S. Jacobsen \& U. Kück, (1997) An efficient procedure to isolate fungal genes from an indexed cosmid library. J Microbiol Meth 29: 49-61.

Pöggeler, S., M. Nowrousian \& U. Kück, (2006) Fruiting-body development in ascomycetes. In: The Mycota I. K. Esser (ed). Berlin, Heidelberg, New York: Springer, pp. 325-355.

Pöggeler, S., M. Nowrousian, C. Ringelberg, J.J. Loros, J.C. Dunlap \& U. Kück, (2006) Microarray and real time PCR analyses reveal mating type-dependent gene expression in a homothallic fungus. Mol. Genet. Genomics 275: 492-503.

Pöggeler, S., S. Risch, U. Kück \& H.D. Osiewacz, (1997) Mating-type genes from the homothallic fungus Sordaria macrospora are functionally expressed in a heterothallic ascomycete. Genetics 147: 567-580.

Pombo, C.M., J.V. Bonventre, A. Molnar, J. Kyriakis \& T. Force, (1996) Activation of a human Ste20-like kinase by oxidant stress defines a novel stress response pathway. The EMBO journal 15: 45374546.

Pombo, C.M., T. Force, J. Kyriakis, E. Nogueira, M. Fidalgo \& J. Zalvide, (2007) The GCK II and III subfamilies of the STE20 group kinases. Frontiers in bioscience : a journal and virtual library 12: 850-859.

Ponchon, L., C. Dumas, A.V. Kajava, D. Fesquet \& A. Padilla, (2004) NMR solution structure of Mob1, a mitotic exit network protein and its interaction with an NDR kinase peptide. Journal of molecular biology 337: 167-182.

Preisinger, C., B. Short, V. De Corte, E. Bruyneel, A. Haas, R. Kopajtich, J. Gettemans \& F.A. Barr, (2004) YSK1 is activated by the Golgi matrix protein GM130 and plays a role in cell migration through its substrate 14-3-3zeta. The Journal of cell biology 164: 1009-1020.

Qian, Z., C. Lin, R. Espinosa, M. LeBeau \& M.R. Rosner, (2001) Cloning and characterization of MST4, a novel Ste20-like kinase. The Journal of biological chemistry 276: 22439-22445.

Rapoport, T.A., (2007) Protein translocation across the eukaryotic endoplasmic reticulum and bacterial plasma membranes. Nature 450: 663-669.

Raz, L., M.M. Khan, V.B. Mahesh, R.K. Vadlamudi \& D.W. Brann, (2008) Rapid estrogen signaling in the brain. Neuro-Signals 16: 140-153.

Raza, H., (2011) Dual localization of glutathione S-transferase in the cytosol and mitochondria: implications in oxidative stress, toxicity and disease. FEBS Journal 278: 4243-4251.

Read, N.D., A. Fleißner \& N.L. Glass, (2010) Hyphal Fusion. ASM Press, Washington. 
Rech, C., I. Engh \& U. Kuck, (2007) Detection of hyphal fusion in filamentous fungi using differently fluorescence-labeled histones. Current genetics 52: 259-266.

Record, C.J., A. Chaikuad, P. Rellos, S. Das, A.C. Pike, O. Fedorov, B.D. Marsden, S. Knapp \& W.H. Lee, (2010) Structural comparison of human mammalian ste20-like kinases. PloS one 5: e11905.

Ribeiro, P.S., F. Josué, A. Wepf, M.C. Wehr, O. Rinner, G. Kelly, N. Tapon \& M. Gstaiger, (2010) Combined functional genomic and proteomic approaches identify a PP2A complex as a negative regulator of Hippo signaling. Molecular and cellular biology 39: 521-534.

Robinson, P.J., (1997) Signal transduction via GPI-anchored membrane proteins. Advances in experimental medicine and biology 419: 365-370.

Roca, M.G., J. Arlt, C.E. Jeffree \& N.D. Read, (2005) Cell biology of conidial anastomosis tubes in Neurospora crassa. Eukaryotic cell 4: 911-919.

Salcini, A.E., M.A. Hilliard, A. Croce, S. Arbucci, P. Luzzi, C. Tacchetti, L. Daniell, P. De Camilli, P.G. Pelicci, P.P. Di Fiore \& P. Bazzicalupo, (2001) The Eps15 C. elegans homologue EHS-1 is implicated in synaptic vesicle recycling. Nature cell biology 3: 755-760.

Sambrook, J. \& D.W. Russell, (2001) Molecular cloning : a laboratory manual. Cold Spring Harbor Laboratory, Cold Spring Harbor, N.Y.

Schindler, D. \& M. Nowrousian, (2014) The polyketide synthase gene pks4 is essential for sexual development and regulates fruiting body morphology in Sordaria macrospora. Fungal Genetics and Biology 68: 48-59.

Schinkmann, K. \& J. Blenis, (1997) Cloning and characterization of a human STE20-like protein kinase with unusual cofactor requirements. The Journal of biological chemistry 272: 28695-28703.

Schneider, H., M. Errede, N.H. Ulrich, D. Virgintino, K. Frei \& H. Bertalanffy, (2011) Impairment of tight junctions and glucose transport in endothelial cells of human cerebral cavernous malformations. Journal of neuropathology and experimental neurology 70: 417-429.

Schulte, J., K.J. Sepp, R.A. Jorquera, C. Wu, Y. Song, P. Hong \& J.T. Littleton, (2010) DMob4/Phocein regulates synapse formation, axonal transport, and microtubule organization. The Journal of neuroscience : the official journal of the Society for Neuroscience 30: 5189-5203.

Sepp, K.J., P. Hong, S.B. Lizarraga, J.S. Liu, L.A. Mejia, C.A. Walsh \& N. Perrimon, (2008) Identification of neural outgrowth genes using genome-wide RNAi. PLoS genetics 4: e1000111.

Sestak, S., I. Hagen, W. Tanner \& S. Strahl, (2004) Scw10p, a cell-wall glucanase/transglucosidase important for cell-wall stability in Saccharomyces cerevisiae. Microbiology 150: 3197-3208.

Shim, W.B., U.S. Sagaram, Y.E. Choi, J. So, H.H. Wilkinson \& Y.W. Lee, (2006) FSR1 is essential for virulence and female fertility in Fusarium verticillioides and F. graminearum. Molecular plantmicrobe interactions : MPMI 19: 725-733.

Siegel, A.M., H. Bertalanffy, J.J. Dichgans, C.E. Elger, H. Hopf, N. Hopf, M. Keidel, A. Kleider, G. Nowak, R.A. Pfeiffer, J. Schramm, S. Spuck, H. Stefan, U. Sure, C.R. Baumann, G.A. Rouleau, D.J. Verlaan, E. Andermann \& F. Andermann, (2005) Familial cavernous malformations of the central nervous system. A clinical and genetic study of 15 German families. Der Nervenarzt 76: 175-180.

Simonin, A.R., C.G. Rasmussen, M. Yang \& N.L. Glass, (2010) Genes encoding a striatin-like protein (ham3 ) and a forkhead associated protein (ham-4) are required for hyphal fusion in Neurospora crassa. Fungal genetics and biology : FG \& B 47: 855-868.

Simons, K. \& D. Toomre, (2000) Lipid rafts and signal transduction. Nature reviews. Molecular cell biology 1: 31-39.

Singh, N.S., N. Shao, J.R. McLean, M. Sevugan, L. Ren, T.G. Chew, A. Bimbo, R. Sharma, X. Tang, K.L. Gould \& M.K. Balasubramanian, (2011) SIN-inhibitory phosphatase complex promotes Cdc11p dephosphorylation and propagates SIN asymmetry in fission yeast. Current biology : CB 21: 1968-1978. 
Smith, T.F., C. Gaitatzes, K. Saxena \& E.J. Neer, (1999) The WD repeat: a common architecture for diverse functions. Trends in biochemical sciences 24: 181-185.

Stavridi, E.S., K.G. Harris, Y. Huyen, J. Bothos, P.M. Verwoerd, S.E. Stayrook, N.P. Pavletich, P.D. Jeffrey \& F.C. Luca, (2003) Crystal structure of a human Mob1 protein: toward understanding Mobregulated cell cycle pathways. Structure 11: 1163-1170.

Stefanova, I., V. Horejsi, I.J. Ansotegui, W. Knapp \& H. Stockinger, (1991) GPI-anchored cell-surface molecules complexed to protein tyrosine kinases. Science 254: 1016-1019.

Stevens, F.C., (1983) Calmodulin: an introduction. Canadian journal of biochemistry and cell biology 61: 906-910.

Su, Y.C., C. Maurel-Zaffran, J.E. Treisman \& E.Y. Skolnik, (2000) The Ste20 kinase misshapen regulates both photoreceptor axon targeting and dorsal closure, acting downstream of distinct signals. Molecular and cellular biology 20: 4736-4744.

Sugden, P.H., L.J. McGuffin \& A. Clerk, (2013) SOcK, MiSTs, MASK and STicKs: the GCKIII (germinal centre kinase III) kinases and their heterologous protein-protein interactions. Biochemical Journal 454: 13-30.

Sukotjo, C., A.A. Abanmy, T. Ogawa \& I. Nishimura, (2002) Molecular cloning of wound inducible transcript (wit 3.0) differentially expressed in edentulous oral mucosa undergoing tooth extraction wound-healing. Journal of dental research 81: 229-235.

Sunyaev, S.R., F. Eisenhaber, I.V. Rodchenkov, B. Eisenhaber, V.G. Tumanyan \& E.N. Kuznetsov, (1999) PSIC: profile extraction from sequence alignments with position-specific counts of independent observations. Protein engineering 12: 387-394.

Sunyaev, S.R., Eisenhaber F., Rodchenkov I.V., Eisenhaber B., Tumanyan V.G. \& K. E.N., (1999) Prediction of potential GPI-modification sites in proprotein sequences. Protein engineering 12: 387-394.

Tan, B., X. Long, H. Nakshatri, K.P. Nephew \& R.M. Bigsby, (2008) Striatin-3 gamma inhibits estrogen receptor activity by recruiting a protein phosphatase. Journal of molecular endocrinology 40: 199-210.

Tanabe, O., D. Hirata, H. Usui, Y. Nishito, T. Miyakawa, K. Igarashi \& M. Takeda, (2001) Fission yeast homologues of the $\mathrm{B}^{\prime}$ subunit of protein phosphatase $2 \mathrm{~A}$ : multiple roles in mitotic cell division and functional interaction with calcineurin. Genes to cells : devoted to molecular \& cellular mechanisms 6: 455-473.

Tanti, G.K. \& S.K. Goswami, (2014) SG2NA recruits DJ-1 and Akt into the mitochondria and membrane to protect cells from oxidative damage. Free Radical Biology and Medicine 75: 1-13.

Tanti, G.K., N. Singarapu, R. Muthuswami \& S.K. Goswami, (2014) Among the three striatin family members, SG2NA was first to arise during evolution. Front Biosci (Schol Ed) 6: 1-15.

Teichert, I., M. Nowrousian, S. Pöggeler \& U. Kück, (2014) The filamentous fungus Sordaria macrospora as a genetic model to study fruiting-body development. Adv Genet 87: 201-246.

Teichert, I., G. Wolff, U. Kück \& M. Nowrousian, (2012) Combining laser microdissection and RNA-seq to chart the transcriptional landscape of fungal development. BMC genomics 13: 511.

Thomas, J.R., R.A. Dwek \& T.W. Rademacher, (1990) Structure, biosynthesis, and function of glycosylphosphatidylinositols. Biochemistry 29: 5413-5422.

Towbin, H., T. Staehelin \& J. Gordon, (1979) Electrophoretic transfer of proteins from polyacrylamide gels to nitrocellulose sheets: procedure and some applications. Proceedings of the National Academy of Sciences of the United States of America 76: 4350-4354.

Trammell, M.A., N.M. Mahoney, D.A. Agard \& R.D. Vale, (2008) Mob4 plays a role in spindle focusing in Drosophila S2 cells. Journal of cell science 121: 1284-1292.

Tran, H., D. Bustos, R. Yeh, B. Rubinfeld, C. Lam, S. Shriver, I. Zilberleyb, M.W. Lee, L. Phu, A.A. Sarkar, I.E. Zohn, I.E. Wertz, D.S. Kirkpatrick \& P. Polakis, (2013) HectD1 E3 ligase modifies adenomatous 
polyposis coli (APC) with polyubiquitin to promote the APC-axin interaction. The Journal of biological chemistry 288: 3753-3767.

Tsukita, S., S. Yonemura \& S. Tsukita, (1997) ERM proteins: head-to-tail regulation of actin-plasma membrane interaction. Trends in biochemical sciences 22: 53-58.

Tucker, C.L., L.A. Peteya, A.M. Pittman \& J. Zhong, (2009) A genetic test for yeast two-hybrid bait competency using RanBPM. Genetics 182: 1377-1379.

Vaknin, Y., Y. Shadkchan, E. Levdansky, M. Morozov, J. Romano \& N. Osherov, (2014) The three Aspergillus fumigatus CFEM-domain GPI-anchored proteins (CfmA-C) affect cell-wall stability but do not play a role in fungal virulence. Fungal genetics and biology : FG \& B 63: 55-64.

van der Voorn, L. \& H.L. Ploegh, (1992) The WD-40 repeat. FEBS letters 307: 131-134.

Varsano, T., M.Q. Dong, I. Niesman, H. Gacula, X. Lou, T. Ma, J.R. Testa, J.R. Yates, 3rd \& M.G. Farquhar, (2006) GIPC is recruited by APPL to peripheral TrkA endosomes and regulates TrkA trafficking and signaling. Molecular and cellular biology 26: 8942-8952.

Verde, F., D.J. Wiley \& P. Nurse, (1998) Fission yeast orb6, a ser/thr protein kinase related to mammalian rho kinase and myotonic dystrophy kinase, is required for maintenance of cell polarity and coordinates cell morphogenesis with the cell cycle. Proceedings of the National Academy of Sciences of the United States of America 95: 7526-7531.

Verlaan, D.J., J. Roussel, S.B. Laurent, C.E. Elger, A.M. Siegel \& G.A. Rouleau, (2005) CCM3 mutations are uncommon in cerebral cavernous malformations. Neurology 65: 1982-1983.

Vitulo, N., A. Vezzi, G. Galla, S. Citterio, G. Marino, B. Ruperti, M. Zermiani, E. Albertini, G. Valle \& G. Barcaccia, (2007) Characterization and evolution of the cell cycle-associated mob domaincontaining proteins in eukaryotes. Evolutionary bioinformatics online 3: 121-158.

Voigt, O. \& S. Pöggeler, (2013) Autophagy genes Smatg8 and Smatg4 are required for fruiting-body development, vegetative growth and ascospore germination in the filamentous ascomycete Sordaria macrospora. Autophagy 9: 33-49.

Voss, K., S. Stahl, E. Schleider, S. Ullrich, J. Nickel, T.D. Mueller \& U. Felbor, (2007) CCM3 interacts with $\mathrm{CCM} 2$ indicating common pathogenesis for cerebral cavernous malformations. Neurogenetics 8 : 249-256.

Wang, C.L., W.B. Shim \& B.D. Shaw, (2010) Aspergillus nidulans striatin (StrA) mediates sexual development and localizes to the endoplasmic reticulum. Fungal genetics and biology : FG \& $B$ 47: 789-799.

Wang, M., S.U. Amano, R.J. Flach, A. Chawla, M. Aouadi \& M.P. Czech, (2013) Identification of Map4k4 as a novel suppressor of skeletal muscle differentiation. Molecular and cellular biology 33: 678687.

Wansink, D.G., R.E. van Herpen, M.M. Coerwinkel-Driessen, P.J. Groenen, B.A. Hemmings \& B. Wieringa, (2003) Alternative splicing controls myotonic dystrophy protein kinase structure, enzymatic activity, and subcellular localization. Molecular and cellular biology 23: 5489-5501.

Wei, X., T. Shimizu \& Z.C. Lai, (2007) Mob as tumor suppressor is activated by Hippo kinase for growth inhibition in Drosophila. The EMBO journal 26: 1772-1781.

Wielowieyski, P.A., S. Sevinc, R. Guzzo, M. Salih, J.T. Wigle \& B.S. Tuana, (2000) Alternative splicing, expression, and genomic structure of the 3 ' region of the gene encoding the sarcolemmalassociated proteins (SLAPs) defines a novel class of coiled-coil tail-anchored membrane proteins. The Journal of biological chemistry 275: 38474-38481.

Wu, W.K., C.H. Cho, C.W. Lee, D. Fan, K. Wu, J. Yu \& J.J. Sung, (2010) Dysregulation of cellular signaling in gastric cancer. Cancer letters 295: 144-153.

Xiang, Q., C. Rasmussen \& N.L. Glass, (2002) The ham-2 locus, encoding a putative transmembrane protein, is required for hyphal fusion in Neurospora crassa. Genetics 160: 169-180. 
Xu, Y., Y. Xing, Y. Chen, Y. Chao, Z. Lin, E. Fan, J.W. Yu, S. Strack, P.D. Jeffrey \& Y. Shi, (2006) Structure of the protein phosphatase 2A holoenzyme. Cell 127: 1239-1251.

Yla-Herttuala, S., T.T. Rissanen, I. Vajanto \& J. Hartikainen, (2007) Vascular endothelial growth factors: biology and current status of clinical applications in cardiovascular medicine. Journal of the American College of Cardiology 49: 1015-1026.

Yogev, O., A. Naamati \& O. Pines, (2011) Fumarase: a paradigm of dual targeting and dual localized functions. The FEBS journal 278: 4230-4242.

Young, N. \& J. Moss, (2000) PNH and the GPI-Linked Proteins. Academic press.

Yu, X.X., X. Du, C.S. Moreno, R.E. Green, E. Ogris, Q. Feng, L. Chou, M.J. McQuoid \& D.C. Pallas, (2001) Methylation of the protein phosphatase $2 \mathrm{~A}$ catalytic subunit is essential for association of Balpha regulatory subunit but not SG2NA, striatin, or polyomavirus middle tumor antigen. Molecular biology of the cell 12: 185-199.

Zhang, M., L. Dong, Z. Shi, S. Jiao, Z. Zhang, W. Zhang, G. Liu, C. Chen, M. Feng, Q. Hao, W. Wang, M. Yin, Y. Zhao, L. Zhang \& Z. Zhou, (2013) Structural mechanism of CCM3 heterodimerization with GCK III kinases. Structure 21: 680-688.

Zhao, B., K. Tumaneng \& K.-L. Guan, (2011) The Hippo pathway in organ size control, tissue regeneration and stem cell self-renewal. Nature cell biology 13: 877-883.

Zheng, X., C. Xu, A. Di Lorenzo, B. Kleaveland, Z. Zou, C. Seiler, M. Chen, L. Cheng, J. Xiao, J. He, M.A. Pack, W.C. Sessa \& M.L. Kahn, (2010) CCM3 signaling through sterile 20-like kinases plays an essential role during zebrafish cardiovascular development and cerebral cavernous malformations. The Journal of clinical investigation 120: 2795-2804.

Zhou, T.H., K. Ling, J. Guo, H. Zhou, Y.L. Wu, Q. Jing, L. Ma \& G. Pei, (2000) Identification of a human brain-specific isoform of mammalian STE20-like kinase 3 that is regulated by cAMP-dependent protein kinase. The Journal of biological chemistry 275: 2513-2519.

Zhu, W., E.K. Chan, J. Li, P. Hemmerich \& E.M. Tan, (2001) Transcription activating property of autoantigen SG2NA and modulating effect of WD-40 repeats. Experimental cell research 269: 31 
